\title{
Preparation and synthetic applicability of imidazole-containing cyclic iodonium salts.
}

Nikita S. Antonkin, ${ }^{a}$ Yulia A. Vlasenko, ${ }^{\text {a }}$ Akira Yoshimura, ${ }^{a}$ Vladimir I. Smirnov, ${ }^{\text {b }}$ Tatyana N. Borodina, ${ }^{\mathrm{b}}$ Viktor V. Zhdankin, ${ }^{\mathrm{a}, \mathrm{c}}$ Mekhman S. Yusubov, ${ }^{\mathrm{a} *}$ Alexandr Shafir, ${ }^{\mathrm{d}^{*}}$ Pavel S. Postnikov. ${ }^{\mathrm{a}, \mathrm{e}^{*}}$

a Research School of Chemistry and Applied Biomedical Sciences, Tomsk Polytechnic University, Tomsk 634050, Russian Federation; e-mail: yusubov@mail.ru; postnikov@tpu.ru

${ }^{b}$ A. E. Favorsky Irkutsk Institute of Chemistry, Siberian Branch of the Russian Academy of Sciences, Favorsky Str., 1, Irkutsk 664033, Russian Federation

c Department of Chemistry and Biochemistry, University of Minnesota Duluth, Duluth, Minnesota 55812, USA

${ }^{d}$ Department of Biological Chemistry, IQAC-CSIC, c/Jordi Girona 18-26, Barcelona 08034, Spain; e-mail: alexandr.shafir@iqac.csic.es

e Department of Solid-State Engineering, University of Chemistry and Technology, Prague 16628, Czech Republic

\section{Content}

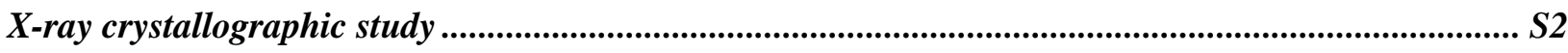

Spectral data 


\section{X-ray crystallographic study}

Crystal data and experimental details are given in Table S1. Table S1 contains CCDC reference number of the supplementary crystallographic data for this paper. These data can be obtained free of charge from The Cambridge Crystallographic Data Centre via http://www.ccdc.cam.ac.uk

Table S1. X-ray crystallographic data for compounds $\mathbf{3 c}$ and $\mathbf{3 j}$.

\begin{tabular}{|c|c|c|}
\hline Compound & $3 c$ & $3 j$ \\
\hline CCDC number & 2058420 & 2058419 \\
\hline Empirical formula & $2\left(\mathrm{C}_{9} \mathrm{H}_{5} \mathrm{FIN}_{2}\right)^{+}, \mathrm{SO}_{4}{ }^{2-}, \mathrm{H}_{2} \mathrm{O}$ & $2\left(\mathrm{C}_{9} \mathrm{H}_{5} \mathrm{BrIN}_{2}\right)^{+}, \mathrm{SO}_{4}{ }^{2-}, 2\left(\mathrm{H}_{2} \mathrm{O}\right)$ \\
\hline $\begin{array}{l}\text { Formula weight / } \\
\mathrm{g} \cdot \mathrm{mol}^{-1}\end{array}$ & 688.18 & 827.99 \\
\hline Crystal system & triclinic & triclinic \\
\hline Space group & $P-1$ & $P-1$ \\
\hline$a / \AA$ & $7.0990(4)$ & $6.993(4)$ \\
\hline$b / \AA$ & $10.1557(6)$ & $10.042(5)$ \\
\hline$c / \AA$ & $14.6998(9)$ & $16.288(9)$ \\
\hline$\alpha, \beta, \gamma /^{\circ}$ & $94.702(2), 93.693(2), 109.939(2)$ & $94.312(16), 91.04(3), 105.0330(19)$ \\
\hline Volume / $\AA^{3}$ & $988.07(10)$ & $1100.6(10)$ \\
\hline$Z$ & 2 & 2 \\
\hline $\begin{array}{l}\text { Density (calculated) } \\
/ \mathrm{g} \cdot \mathrm{cm}^{-3}\end{array}$ & 2.313 & 2.498 \\
\hline $\begin{array}{l}\text { Absorptions } \\
\text { coefficient } / \mathrm{mm}^{-1}\end{array}$ & 3.350 & 6.629 \\
\hline Radiation $(\lambda / \AA)$ & $\operatorname{MoK} \alpha(0.71073)$ & $\operatorname{MoK} \alpha(0.71073)$ \\
\hline Temperature / K & $296(2)$ & 293(2) \\
\hline $2 \Theta$ range $/{ }^{\circ}$ & $2.43-30.07$ & $2.11-30.08$ \\
\hline Crystal size / mm & $0.60 \times 0.19 \times 0.08$ & $0.14 \times 0.08 \times 0.05$ \\
\hline Crystal habit & colourless, needle & colourless, prism \\
\hline $\mathrm{F}(000)$ & 656 & 780 \\
\hline $\begin{array}{l}\text { Reflections } \\
\text { collected }\end{array}$ & 60166 & 69845 \\
\hline $\begin{array}{l}\text { Independent } \\
\text { reflections }\end{array}$ & $4383[\mathrm{R}($ int $)=0.0374]$ & $5731[\mathrm{R}($ int $)=0.0290]$ \\
\hline $\begin{array}{l}\text { Number of ref. } \\
\text { parameters }\end{array}$ & 292 & 314 \\
\hline$R_{1} / w R_{2} \quad[I>2 \sigma(I)]$ & $0.0187 / 0.0517$ & $0.0181 / 0.0379$ \\
\hline $\mathrm{R}_{1} / \mathrm{wR}_{2}$ (all data) & $0.0213 / 0.0528$ & $0.0236 / 0.0442$ \\
\hline $\begin{array}{l}\text { Goodness-of-fit on } \\
\mathrm{F}^{2}\end{array}$ & 1.223 & 1.141 \\
\hline Completeness [\%] & 99.7 & 99.8 \\
\hline Weight scheme & $\begin{array}{l}\mathrm{w}=1 /\left[\sigma^{2}\left(\mathrm{~F}_{\mathrm{o}}^{2}\right)+(0.0246 \mathrm{P})^{2}+0.4019 \mathrm{P}\right] \\
\text { where } \mathrm{P}=\left(\mathrm{F}_{\mathrm{o}}^{2}+2 \mathrm{~F}_{\mathrm{c}}^{2}\right) / 3\end{array}$ & $\begin{array}{l}\mathrm{w}=1 /\left[\sigma^{2}\left(\mathrm{~F}_{\mathrm{o}}^{2}\right)+(0.0161 \mathrm{P})^{2}+1.0204 \mathrm{P}\right] \\
\text { where } \mathrm{P}=\left(\mathrm{F}_{\mathrm{o}}^{2}+2 \mathrm{~F}_{\mathrm{c}}^{2}\right) / 3\end{array}$ \\
\hline
\end{tabular}




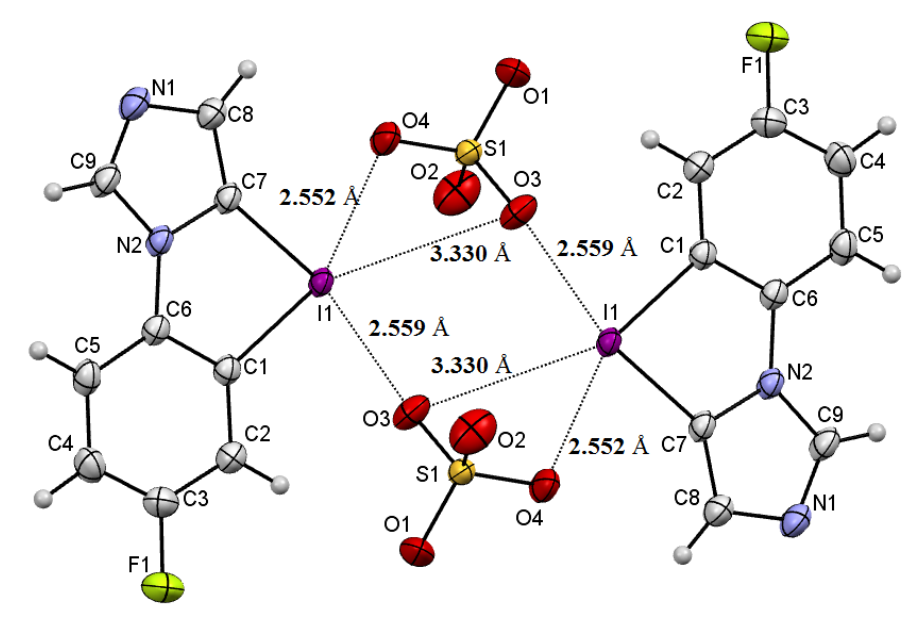

Figure S1. Crystal structure of $3 \boldsymbol{c}$ showing the dimeric nature of the cyclic iodonium salt. Selective distances and angles: $\mathrm{C} 1-\mathrm{I} 1=2.122(2) \AA, \mathrm{C} 7-\mathrm{I} 1=2.077(2) \AA, \mathrm{C} 1-\mathrm{C} 6=1.394(2)$ $\AA, \mathrm{C} 6-\mathrm{N} 2=1.408(3) \AA, \mathrm{C} 7-\mathrm{N} 2=1.388(2) \AA, \angle \mathrm{C} 1-\mathrm{I} 1-\mathrm{C} 7=80.25(8)^{\circ}, \quad \angle \mathrm{I} 1-\mathrm{C} 7-\mathrm{C} 8-\mathrm{H} 8=-$ $5.0^{\circ}$

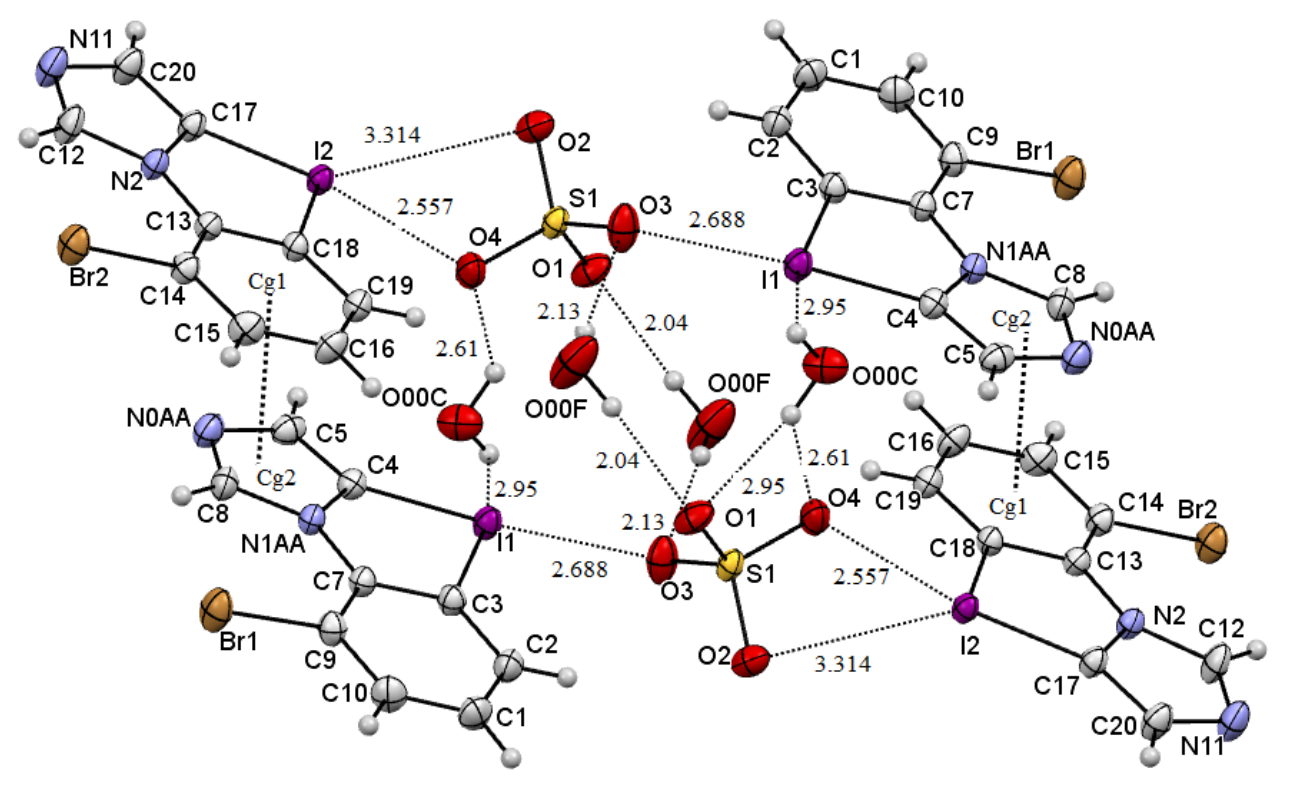

Figure S2. Crystal structure of $\mathbf{3} \boldsymbol{j}$ showing the dimeric nature of the cyclic iodonium salt. Selective distances and angles: $\mathrm{I} 1-\mathrm{C} 3=2.105(2) \AA, \mathrm{I} 1-\mathrm{C} 4=2.058(2) \AA, \mathrm{C} 3-\mathrm{C} 7=1.400(3)$ $\AA, \mathrm{C} 4-\mathrm{N} 1 \mathrm{AA}=1.389(3) \AA, \mathrm{C} 7-\mathrm{N} 1 \mathrm{AA}=1.418(3) \AA, \angle \mathrm{C} 3-\mathrm{I} 1-\mathrm{C} 4=80.23(8)^{\circ}, \angle \mathrm{I} 1-\mathrm{C} 4-\mathrm{C} 5-$ $\mathrm{H} 5=4.5^{\circ} . \mathrm{I} 2-\mathrm{C} 17=2.078(2) \AA, \mathrm{I} 2-\mathrm{C} 18=2.118(2) \AA$, Inter-stacking interactions between iodonium salts: distance between centroids $\mathrm{Cg} 1-\mathrm{Cg} 2=3.550 \AA$. 
Spectral data 


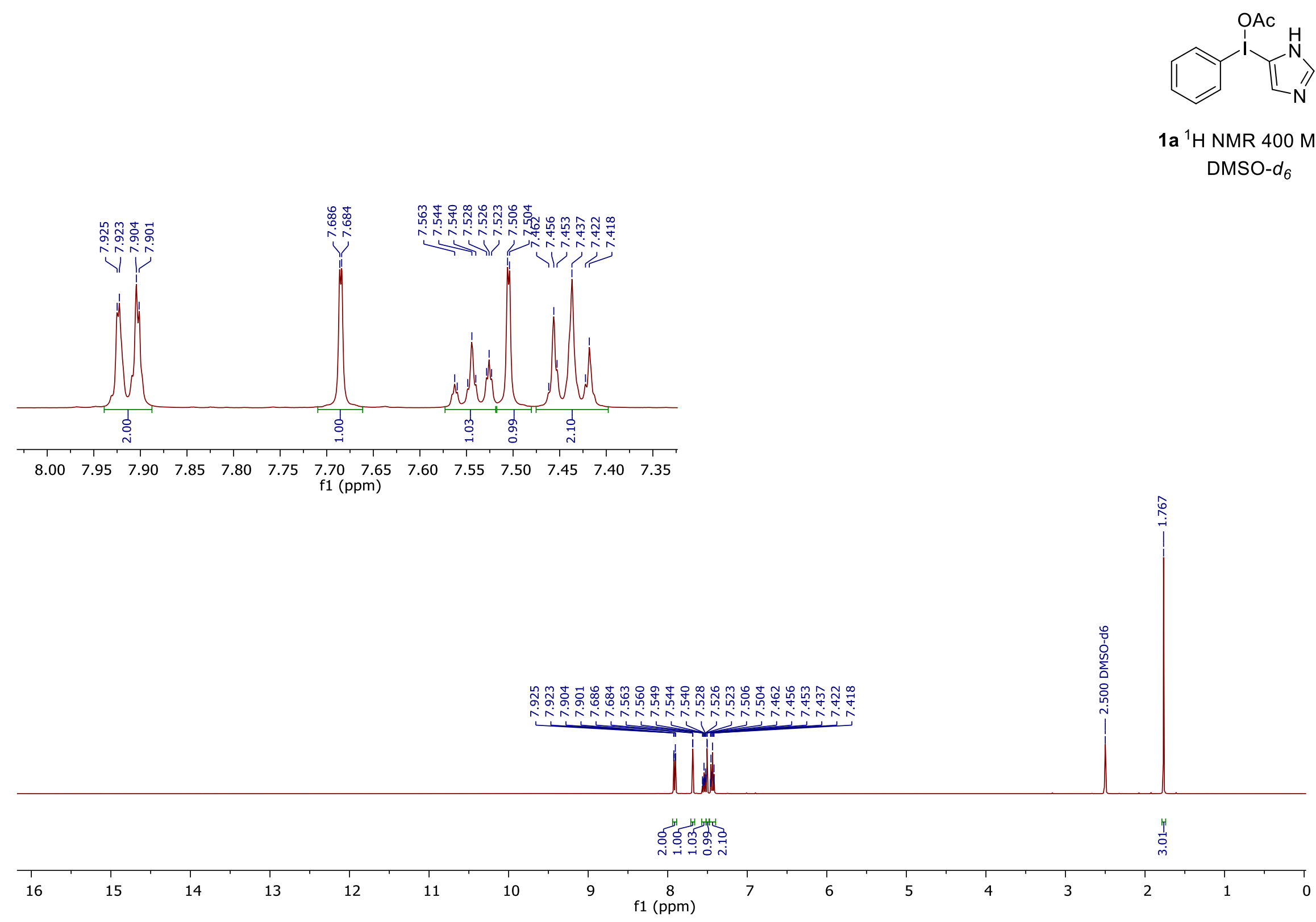



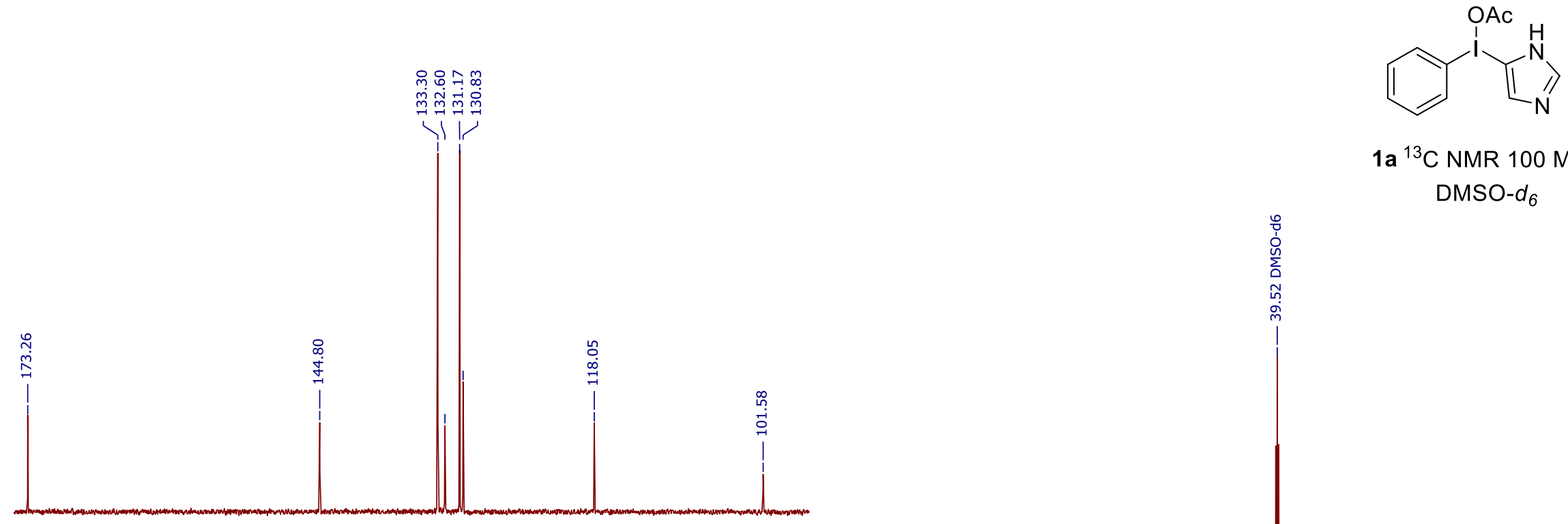

$1 \mathrm{a}^{13} \mathrm{C}$ NMR $100 \mathrm{MHz}$ DMSO-d

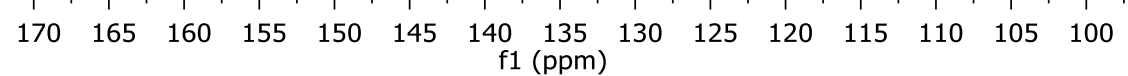

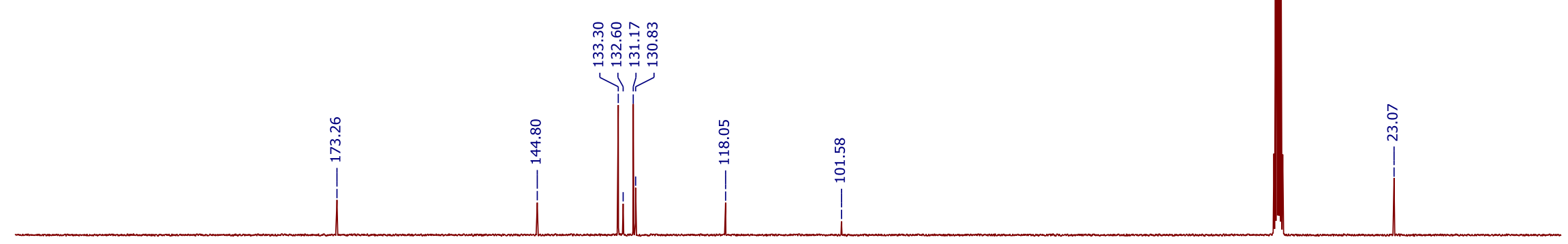

$210 \quad 200 \quad 190 \quad 180$

170

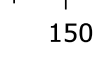

$50 \quad 140$

130

$120 \quad 110 \quad 100$

90

$80 \quad 70$

$60 \quad 50$

$40 \quad 30$

$10 \quad 0$ 


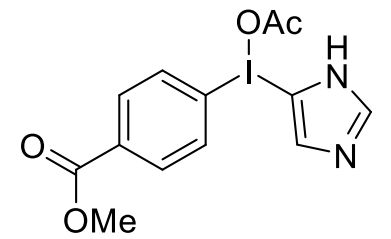

1b ${ }^{1} \mathrm{H}$ NMR $400 \mathrm{MHz}$ $\mathrm{MeOD}-d_{4}$
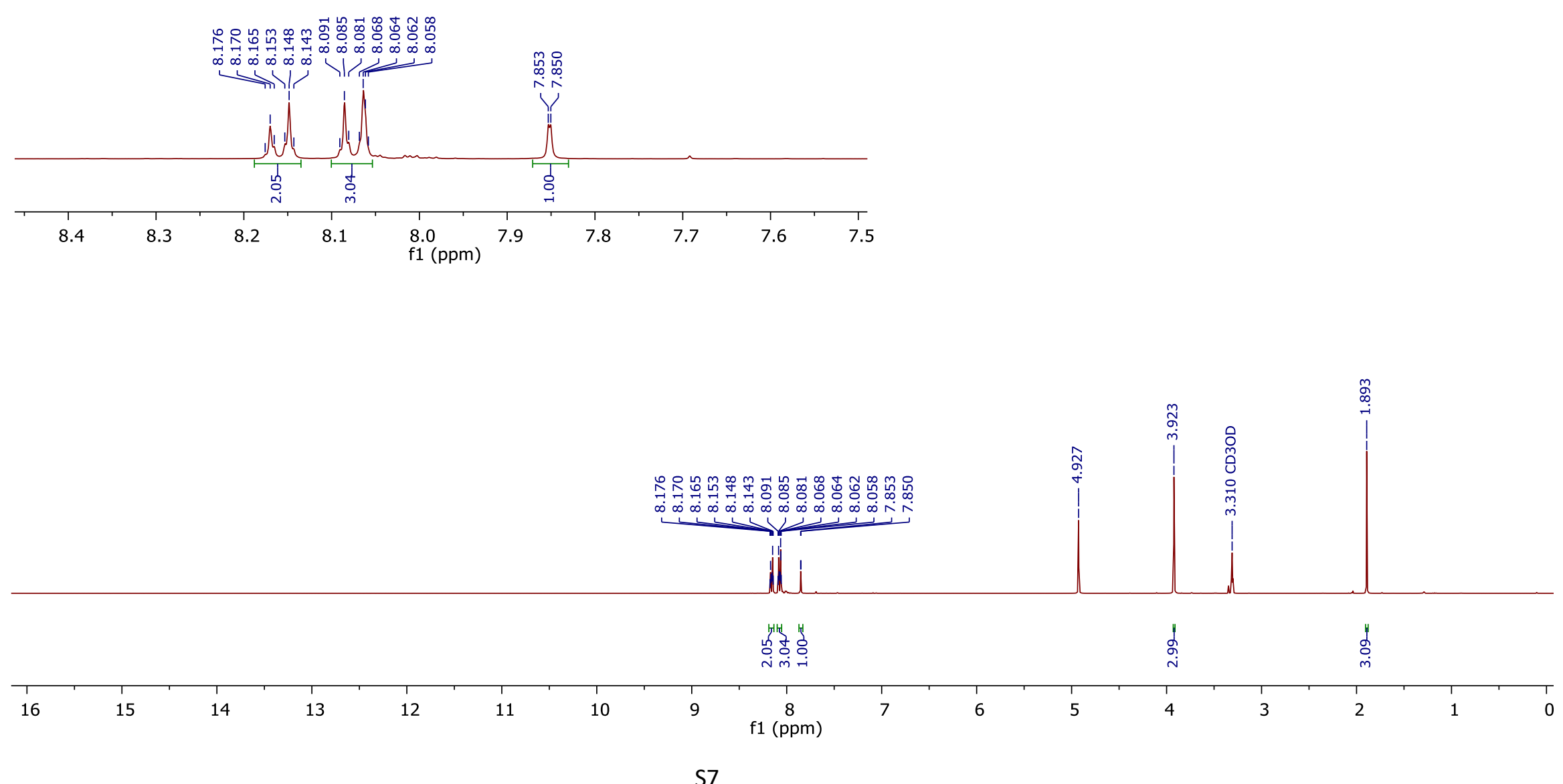

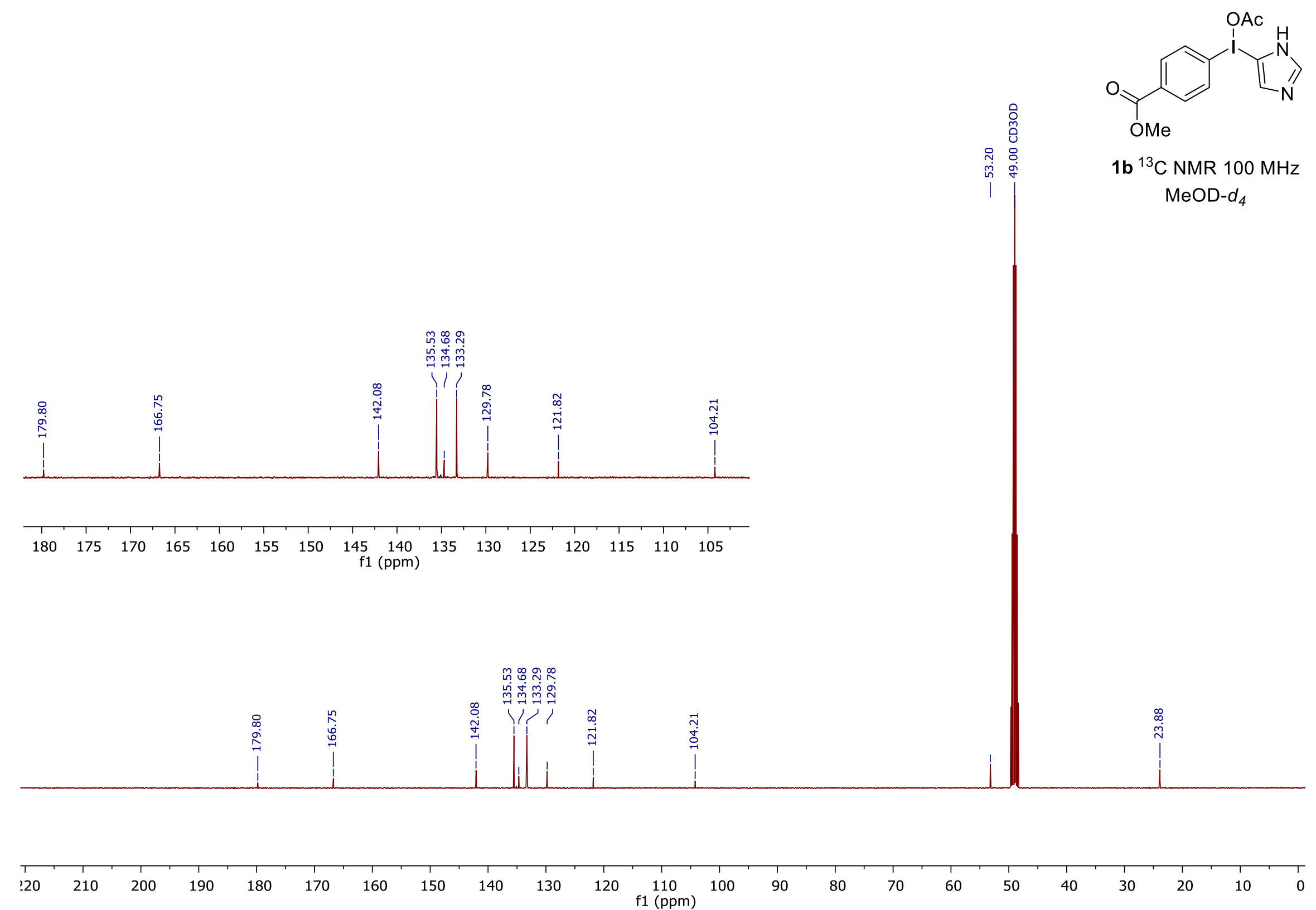


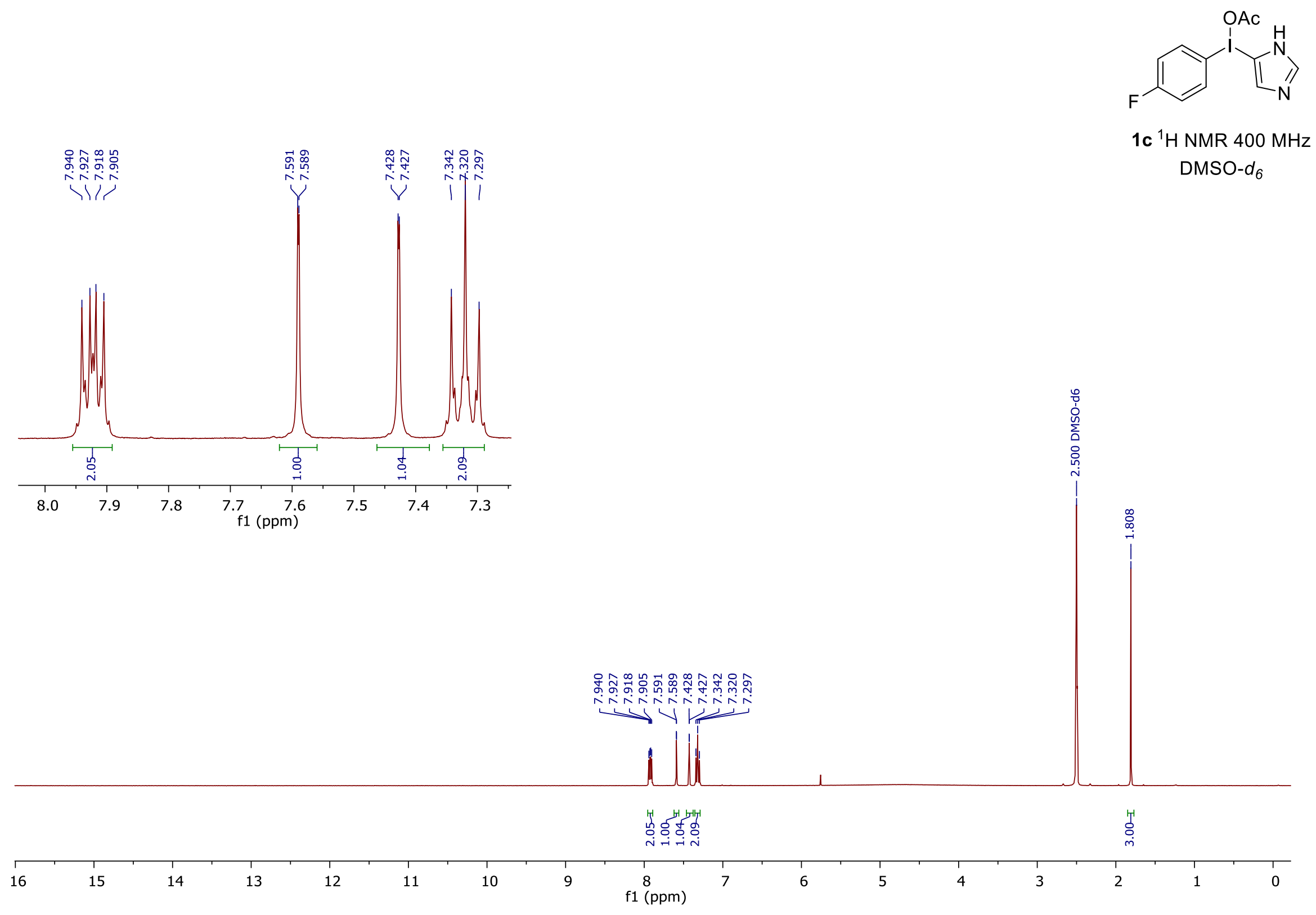



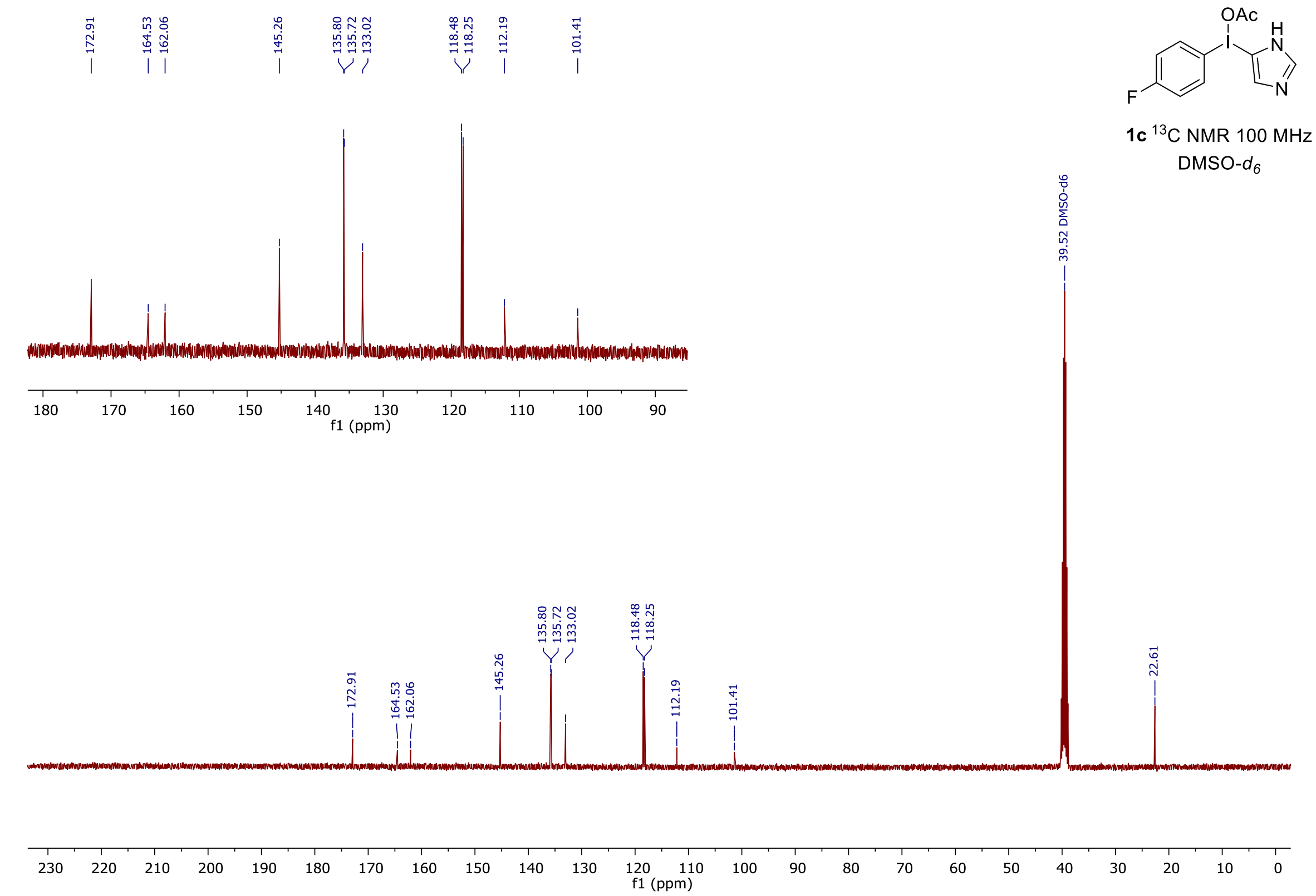


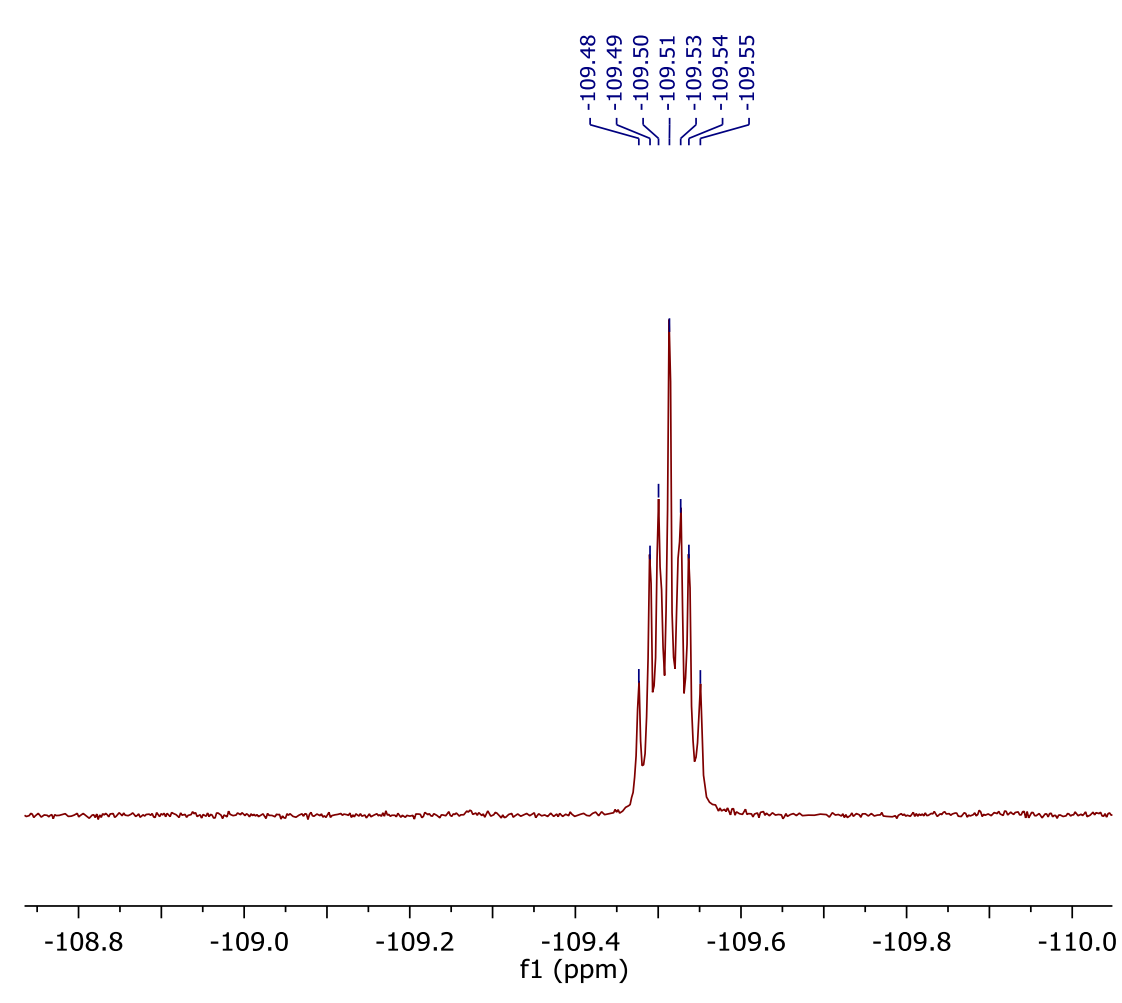




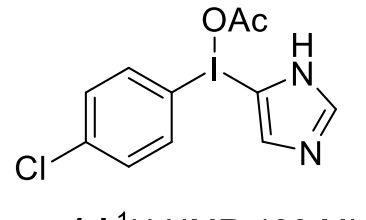

1d ${ }^{1} \mathrm{H}$ NMR $400 \mathrm{MHz}$

MeOD- $d_{4}$
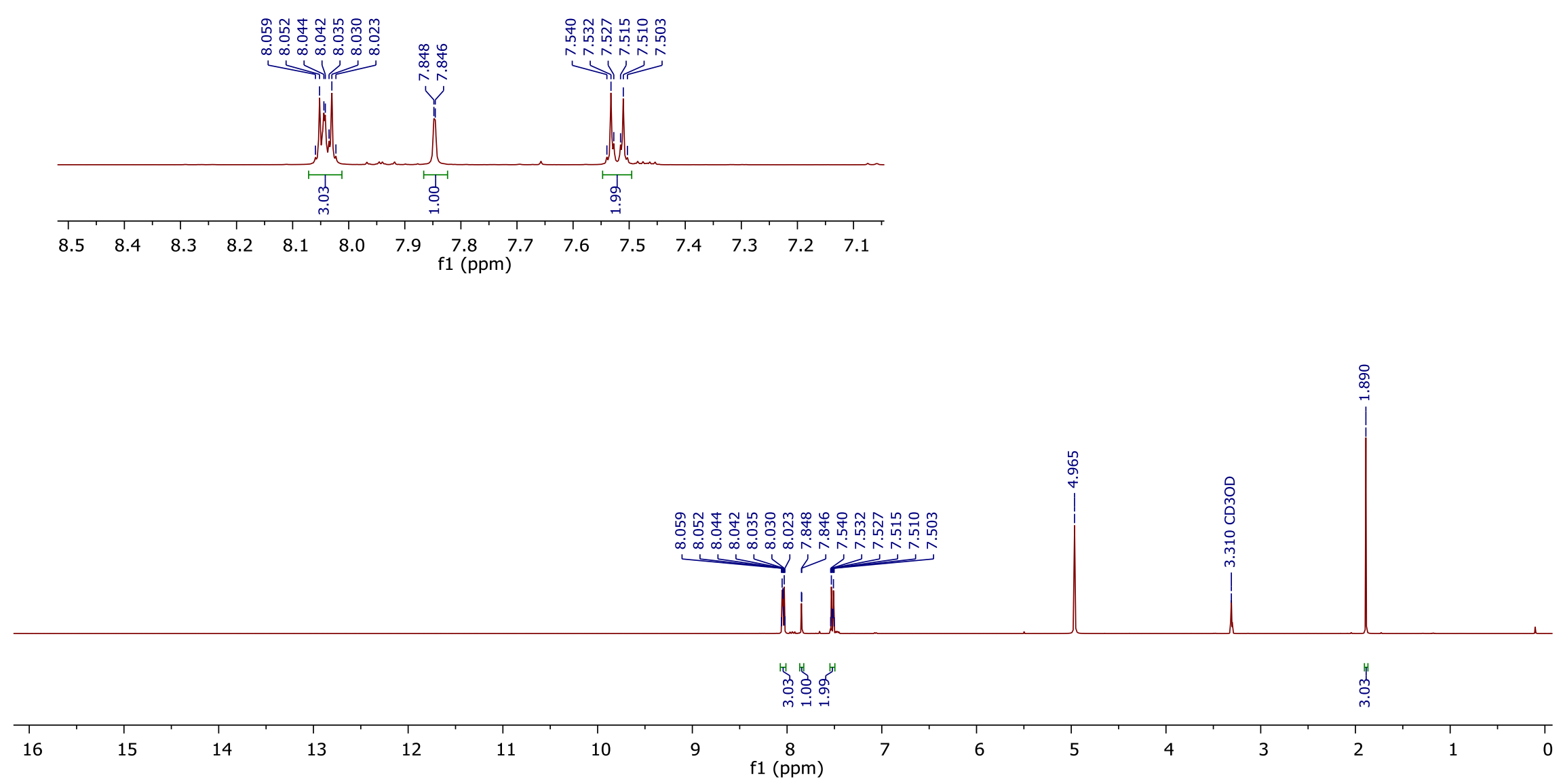

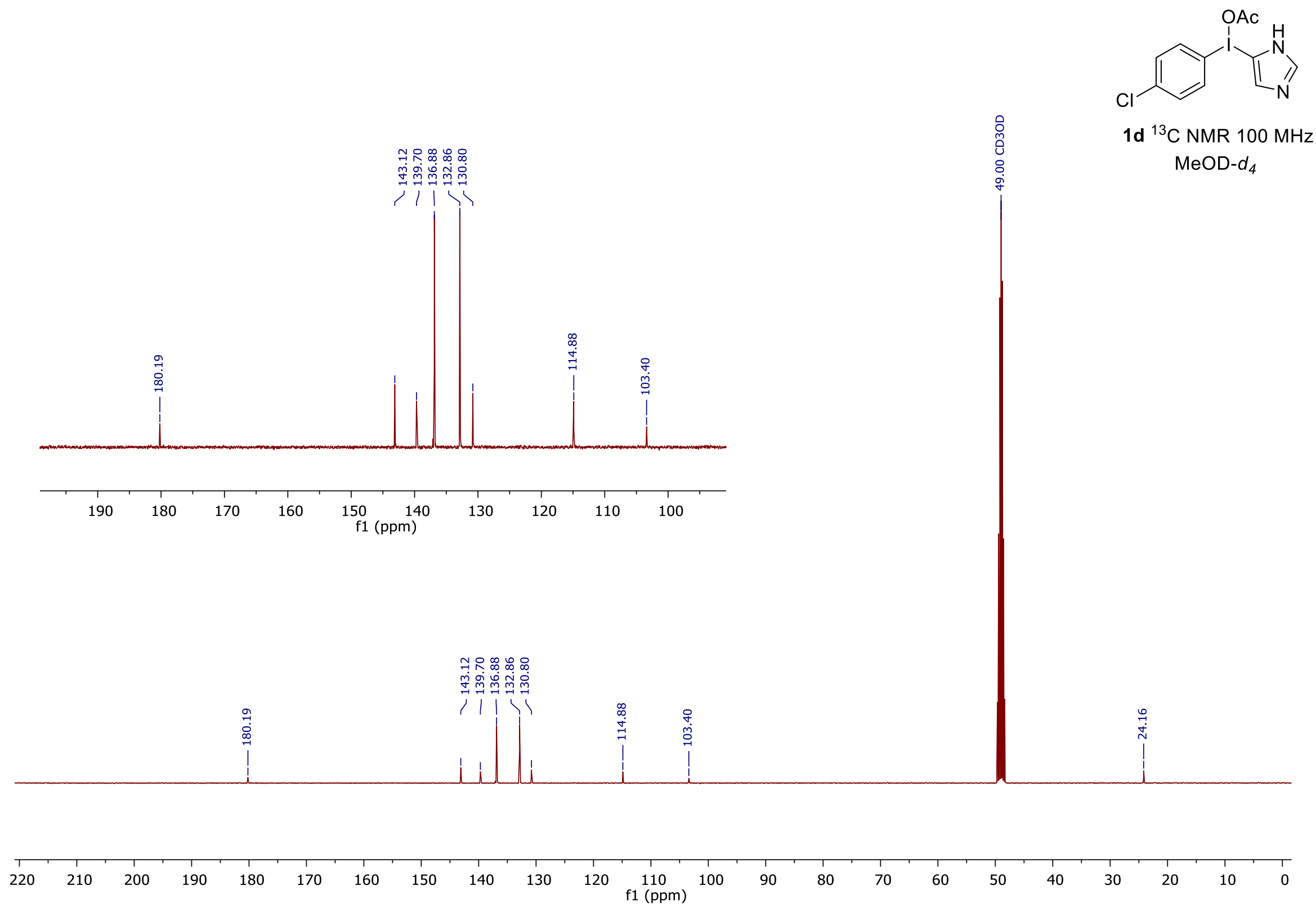


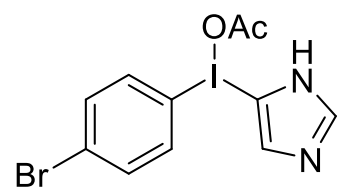

1e ${ }^{1} \mathrm{H}$ NMR $400 \mathrm{MHz}$ MeOD- $d_{4}$
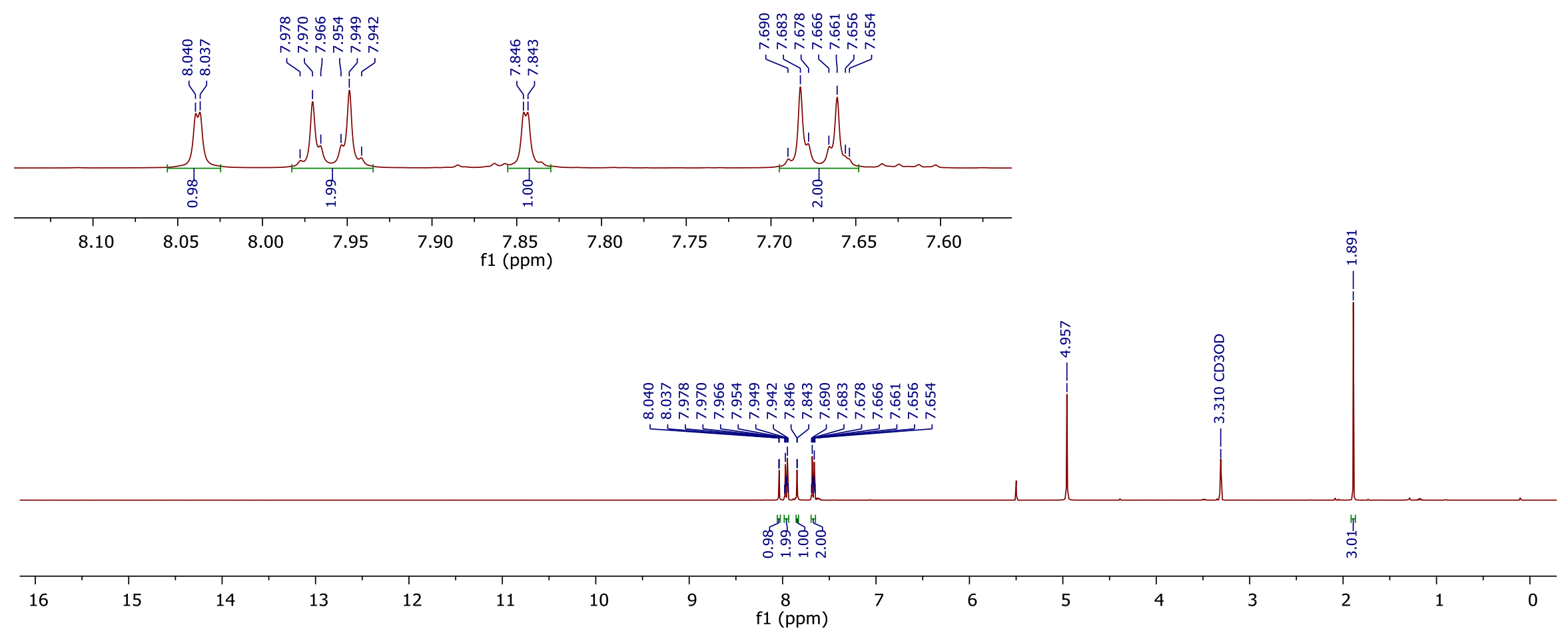

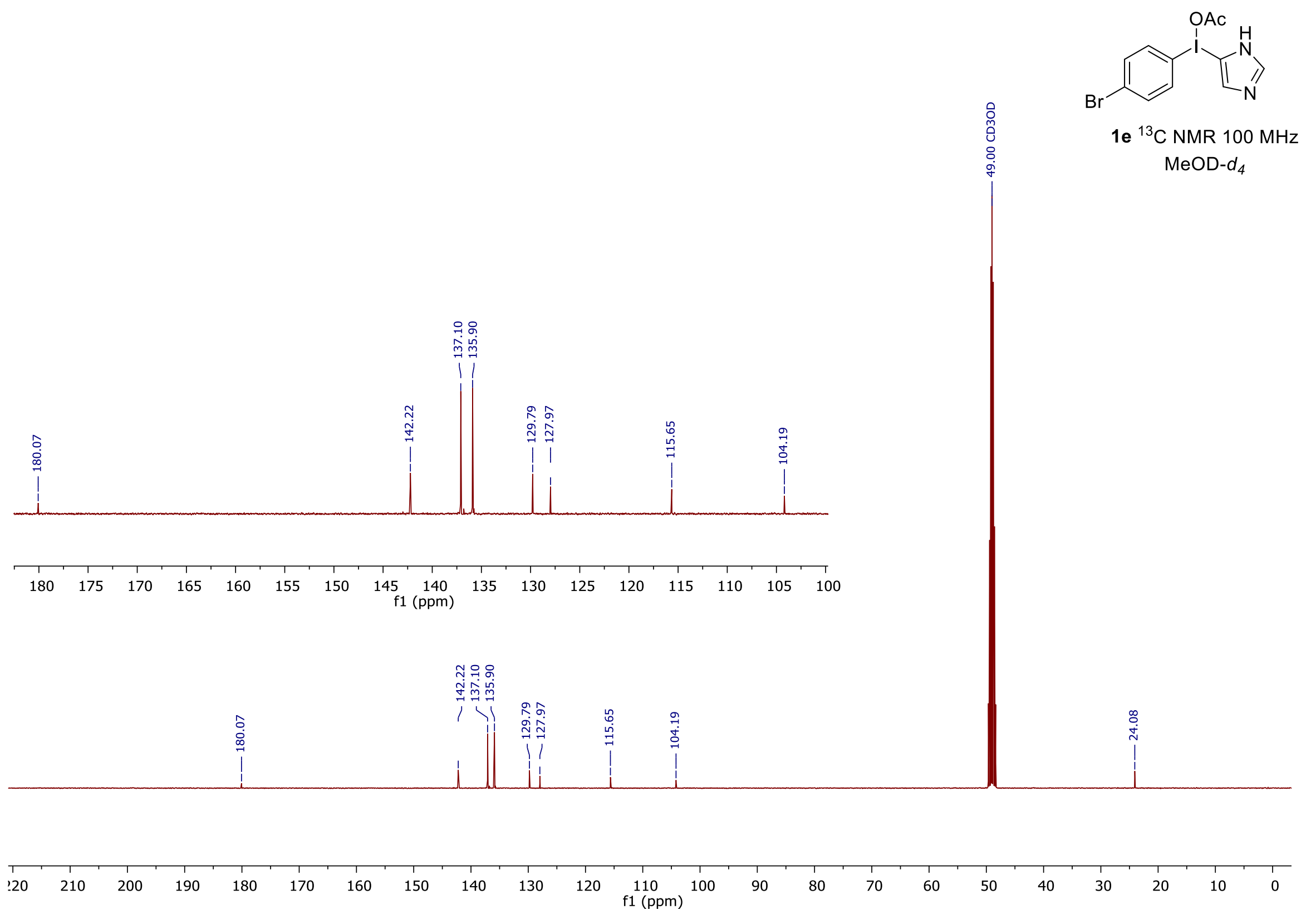

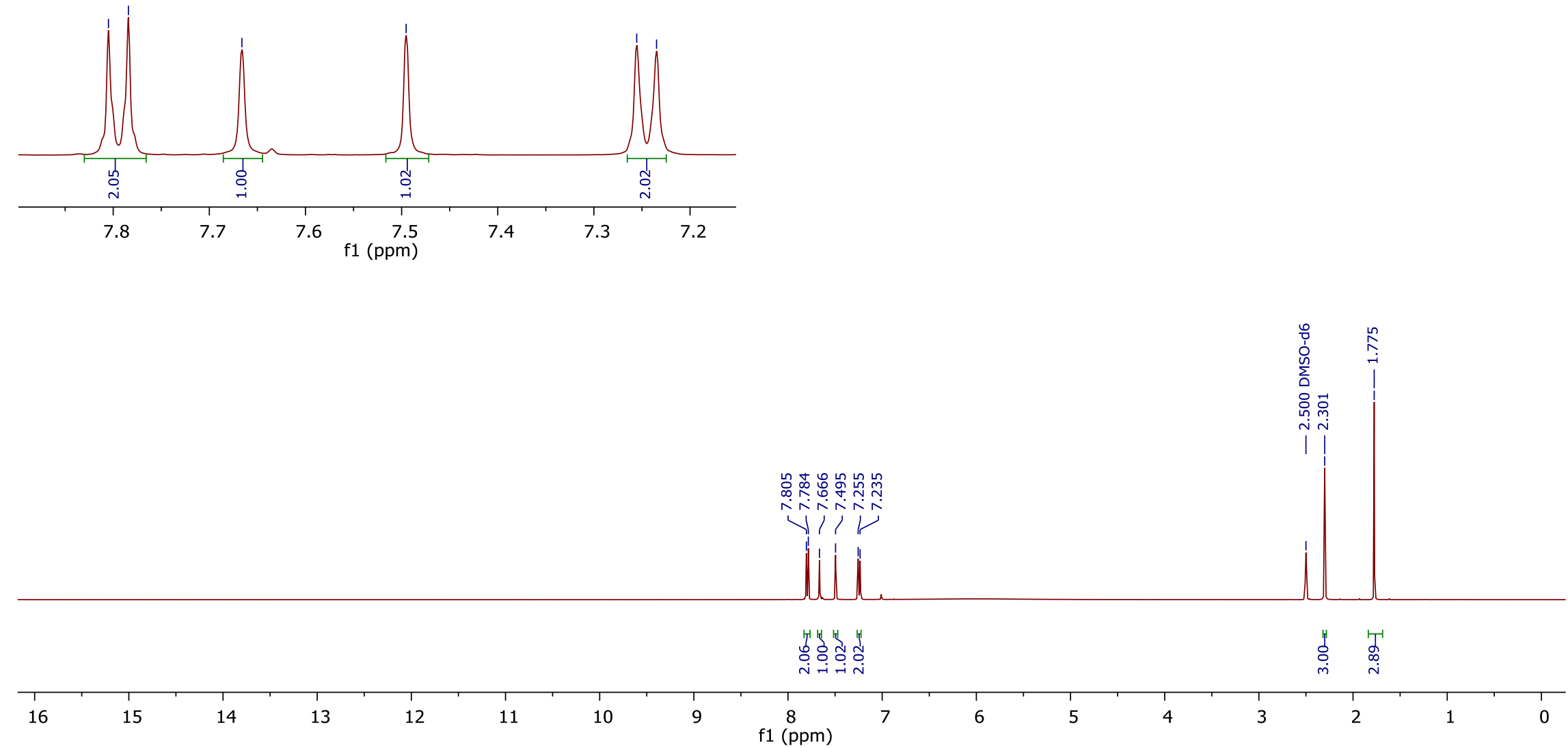

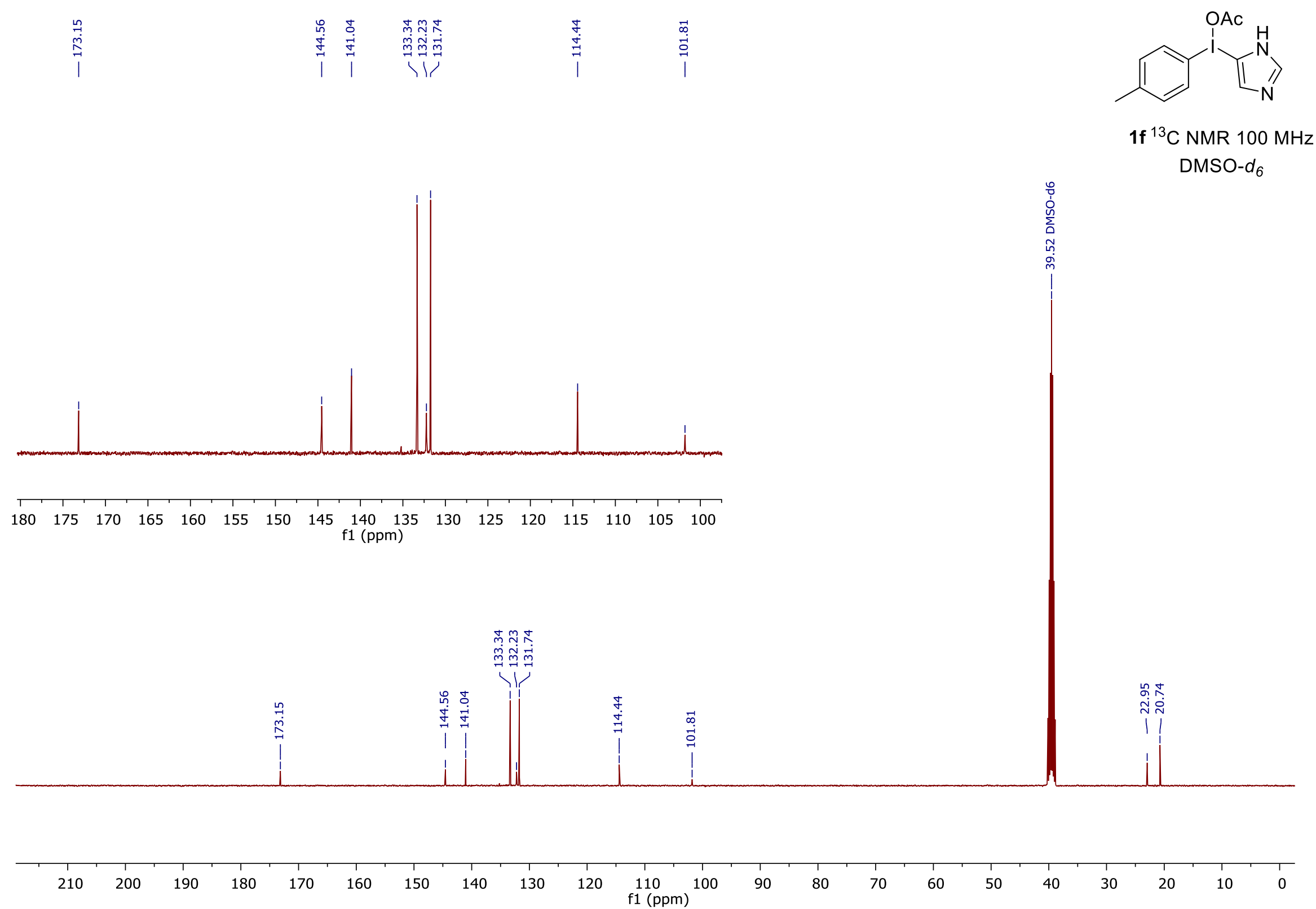


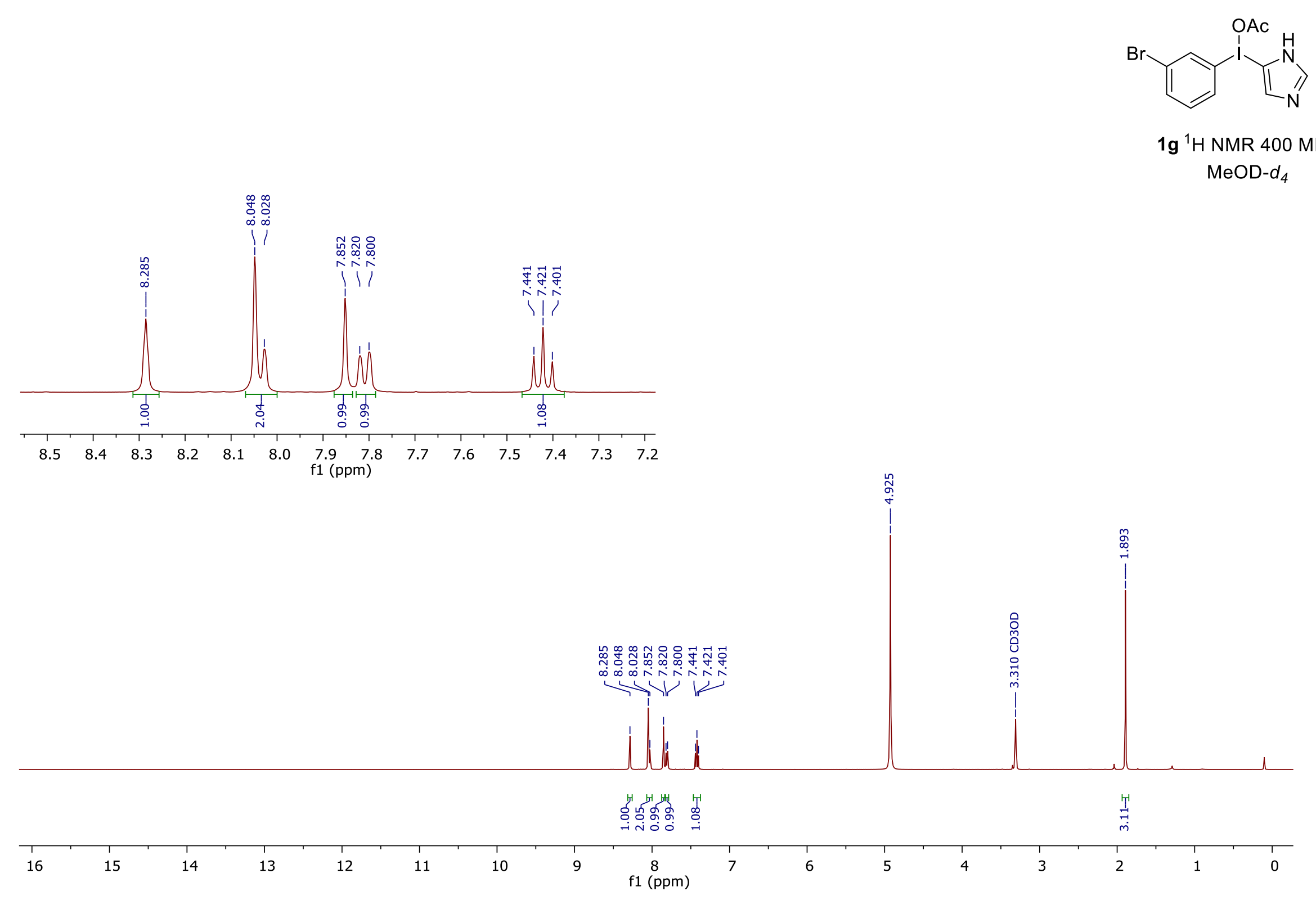




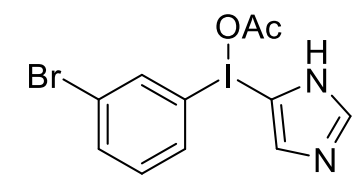

1g ${ }^{13} \mathrm{C}$ NMR $100 \mathrm{MHz}$ MeOD- $d_{4}$
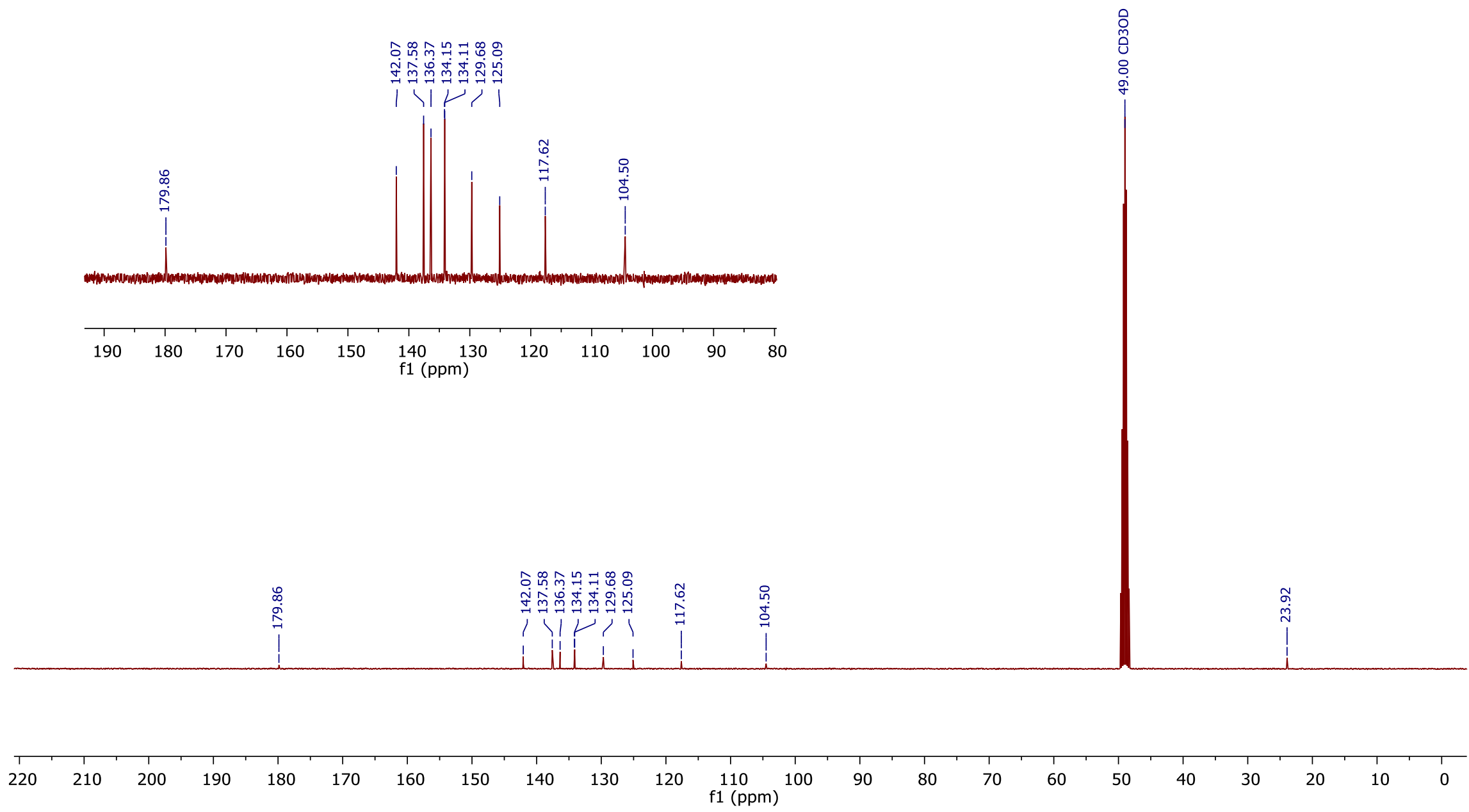

80

$70 \quad 60$

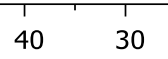

20 


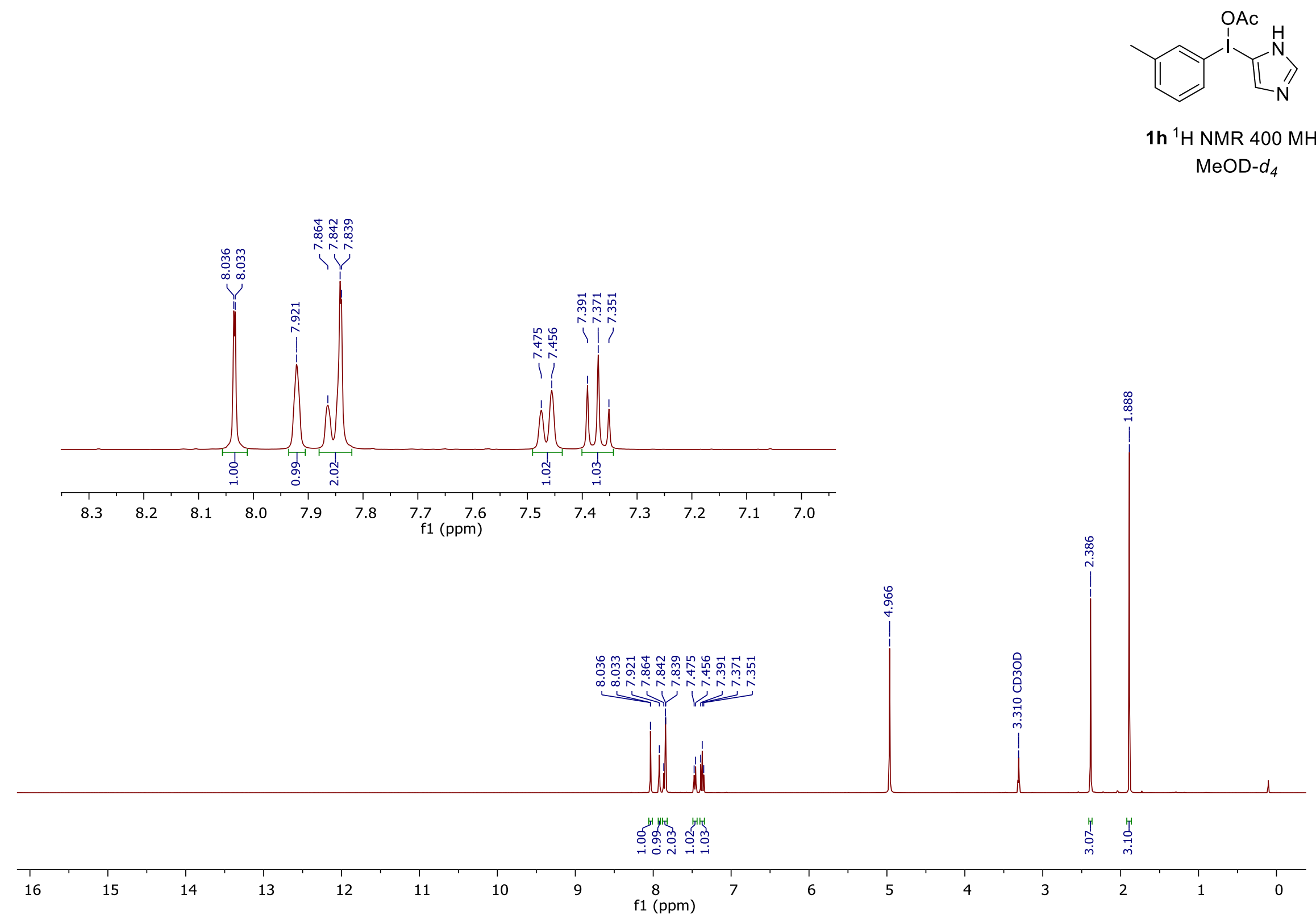



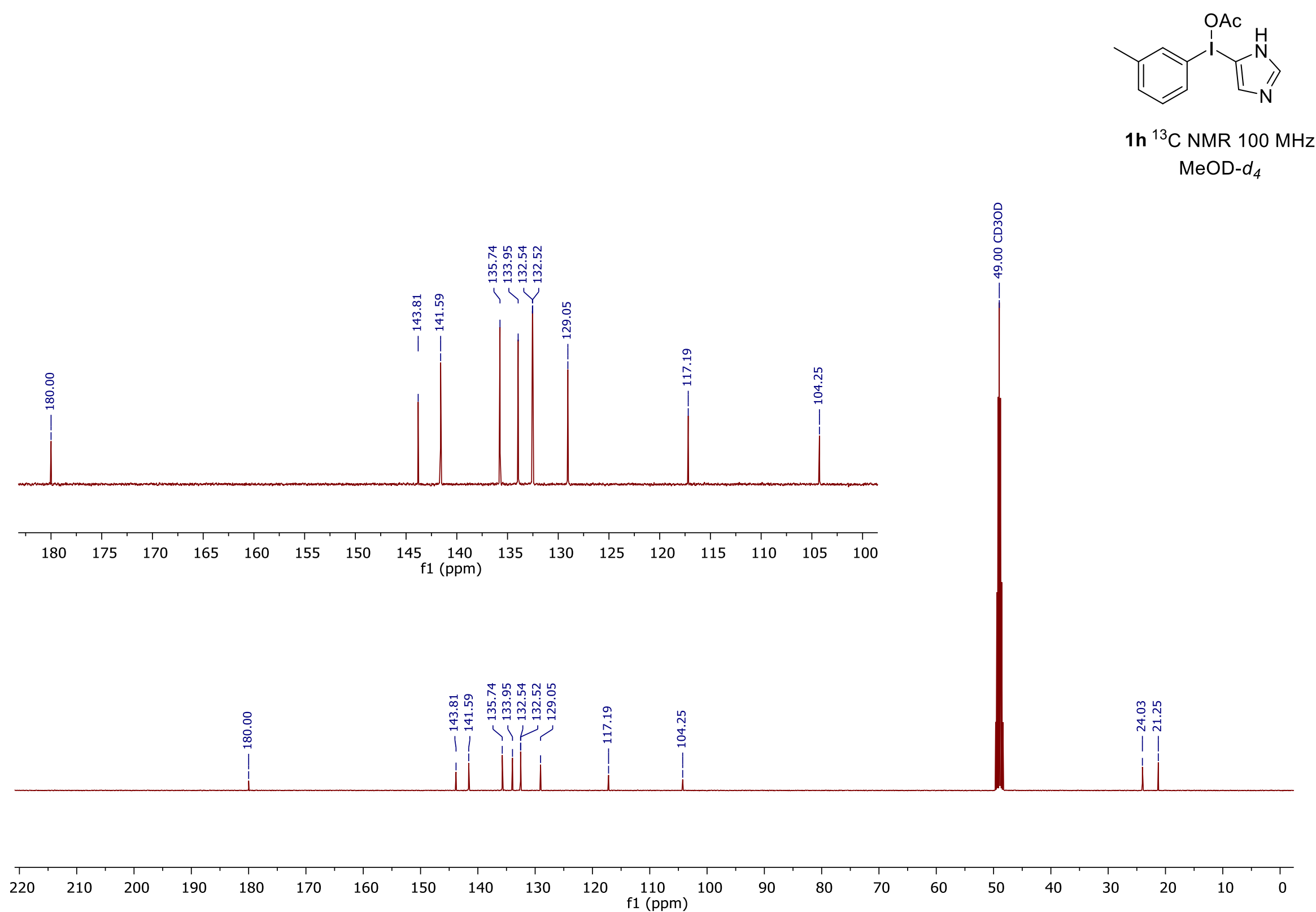

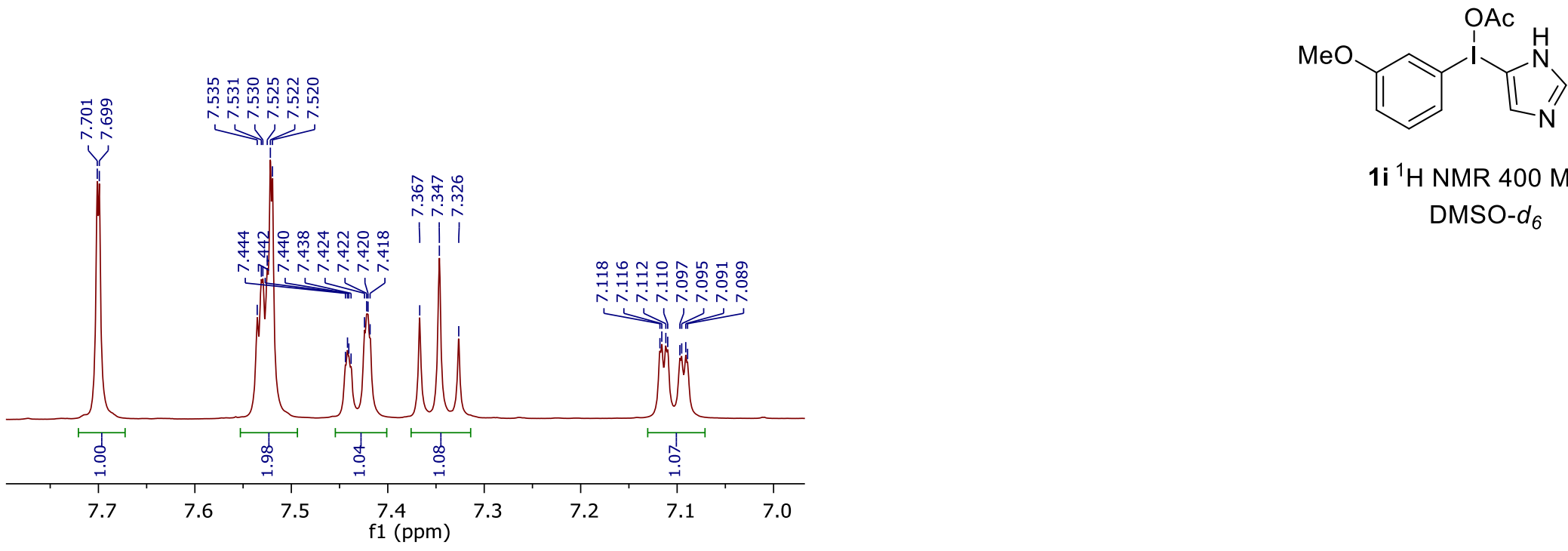

1i ${ }^{1} \mathrm{H}$ NMR $400 \mathrm{MHz}$

DMSO- $d_{6}$

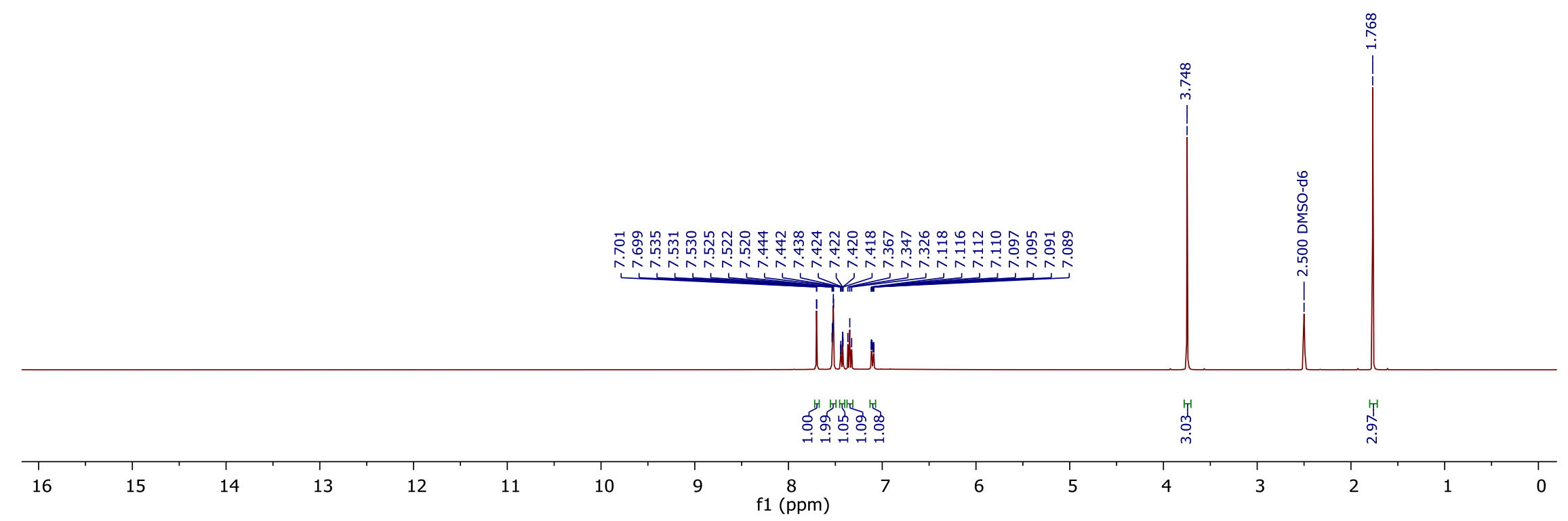




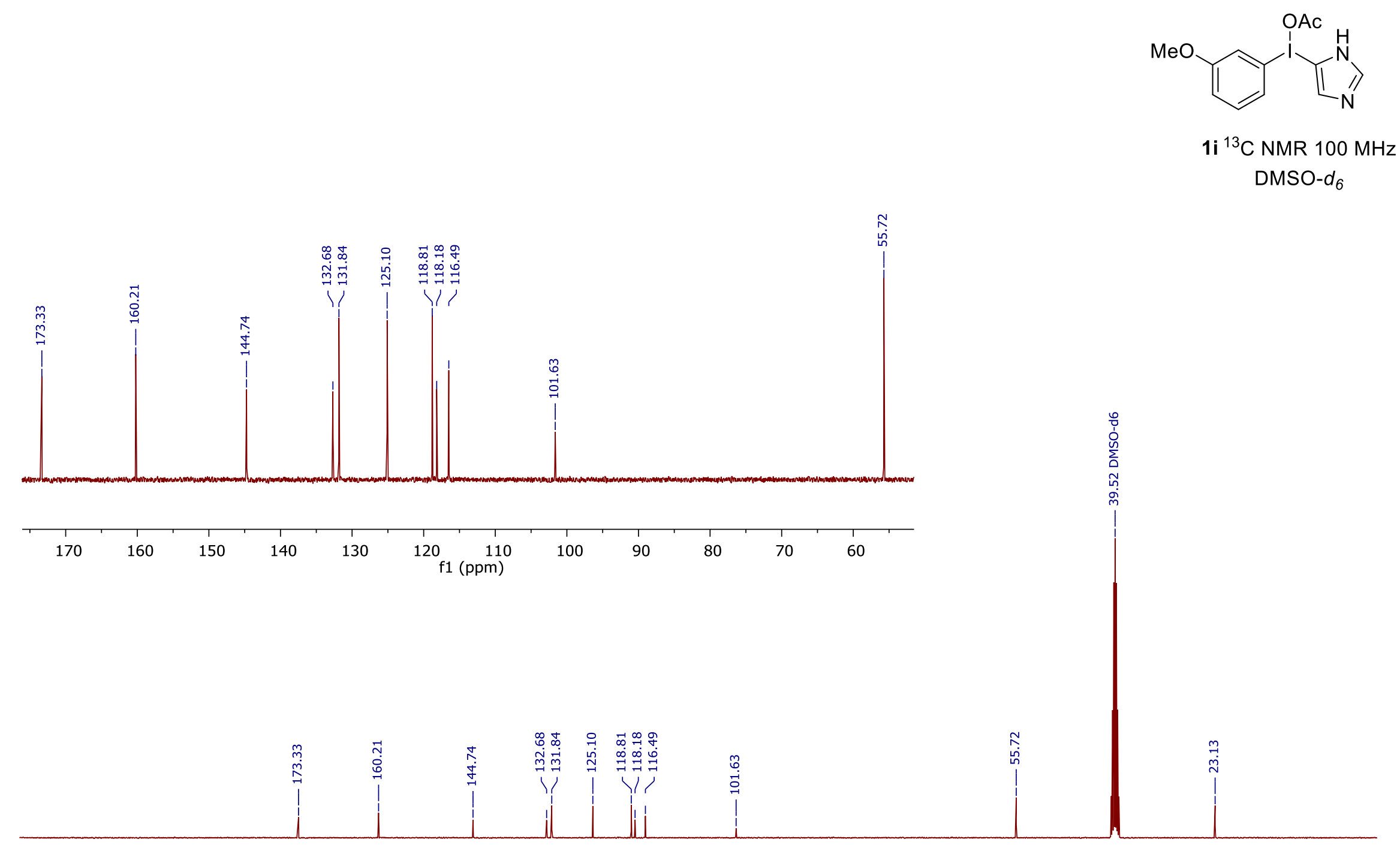

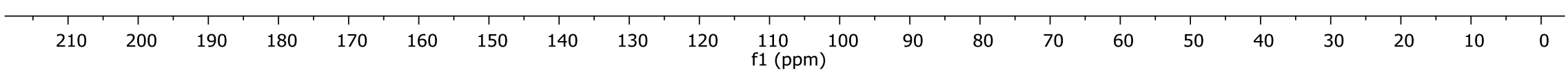




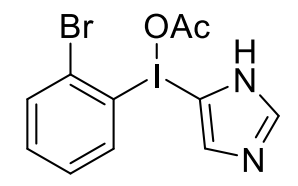

1j ${ }^{1} \mathrm{H}$ NMR $400 \mathrm{MHz}$

MeOD- $d_{4}$
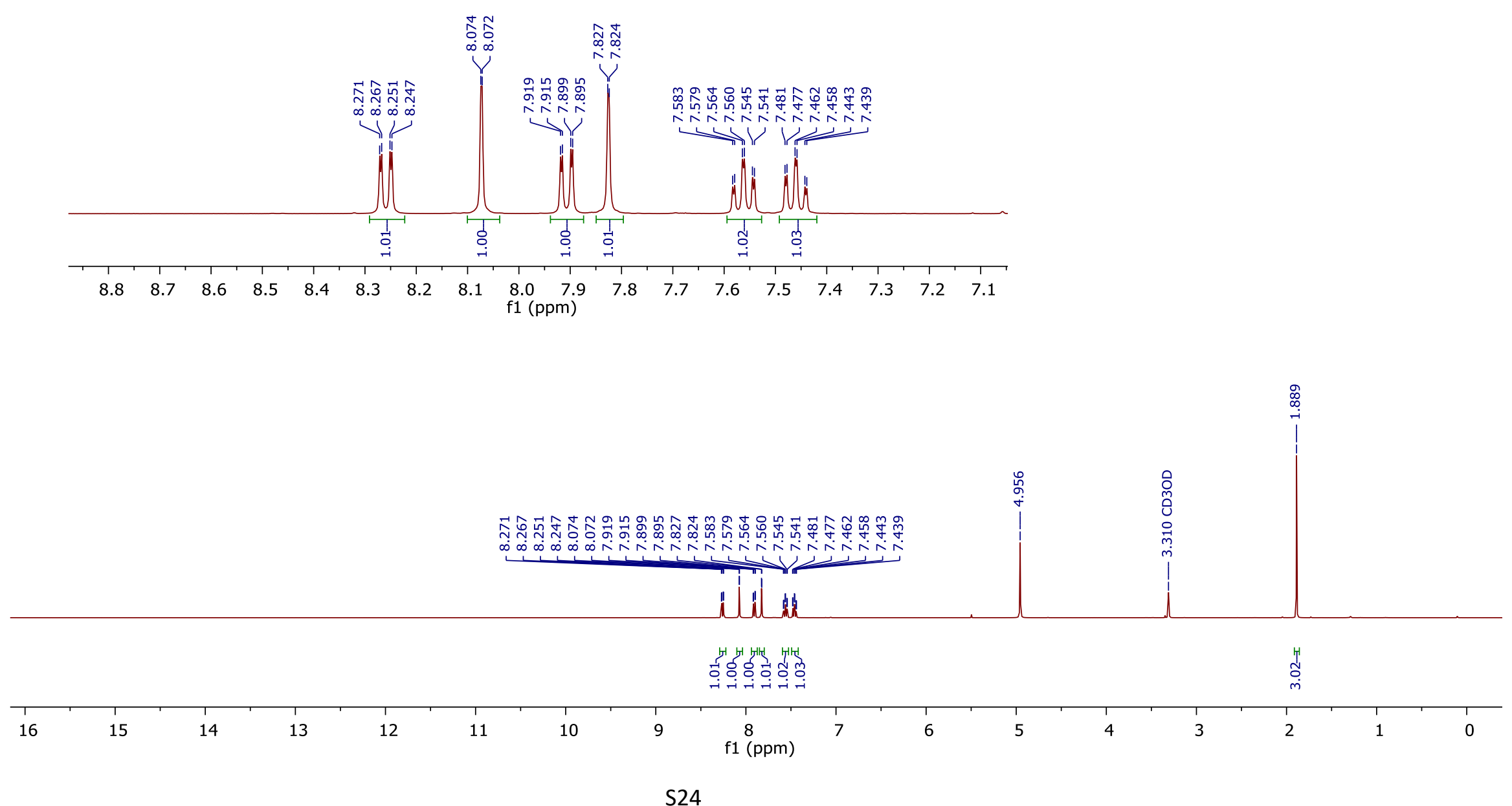

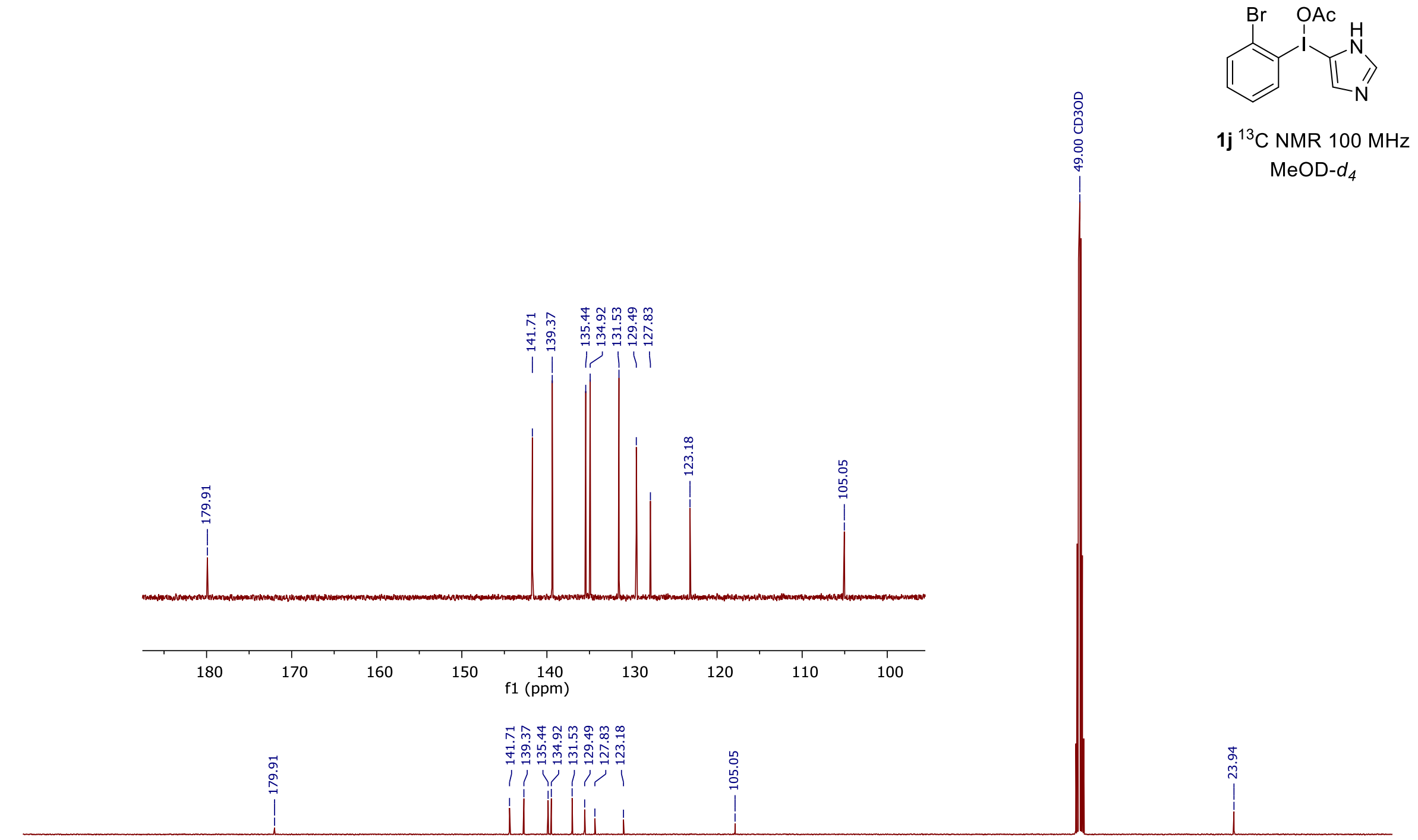

1j ${ }^{13} \mathrm{C}$ NMR $100 \mathrm{MHz}$

$\mathrm{MeOD}-d_{4}$

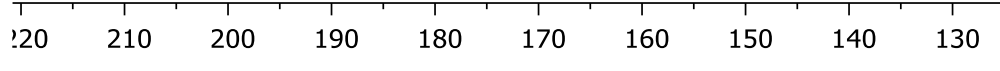

110
$\mathrm{f} 1$ (ppm)

$70 \quad 60$ 


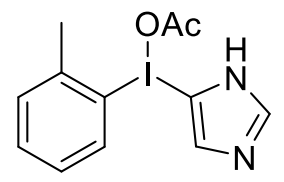

1k ${ }^{1} \mathrm{H}$ NMR $400 \mathrm{MHz}$ MeOD- $d_{4}$
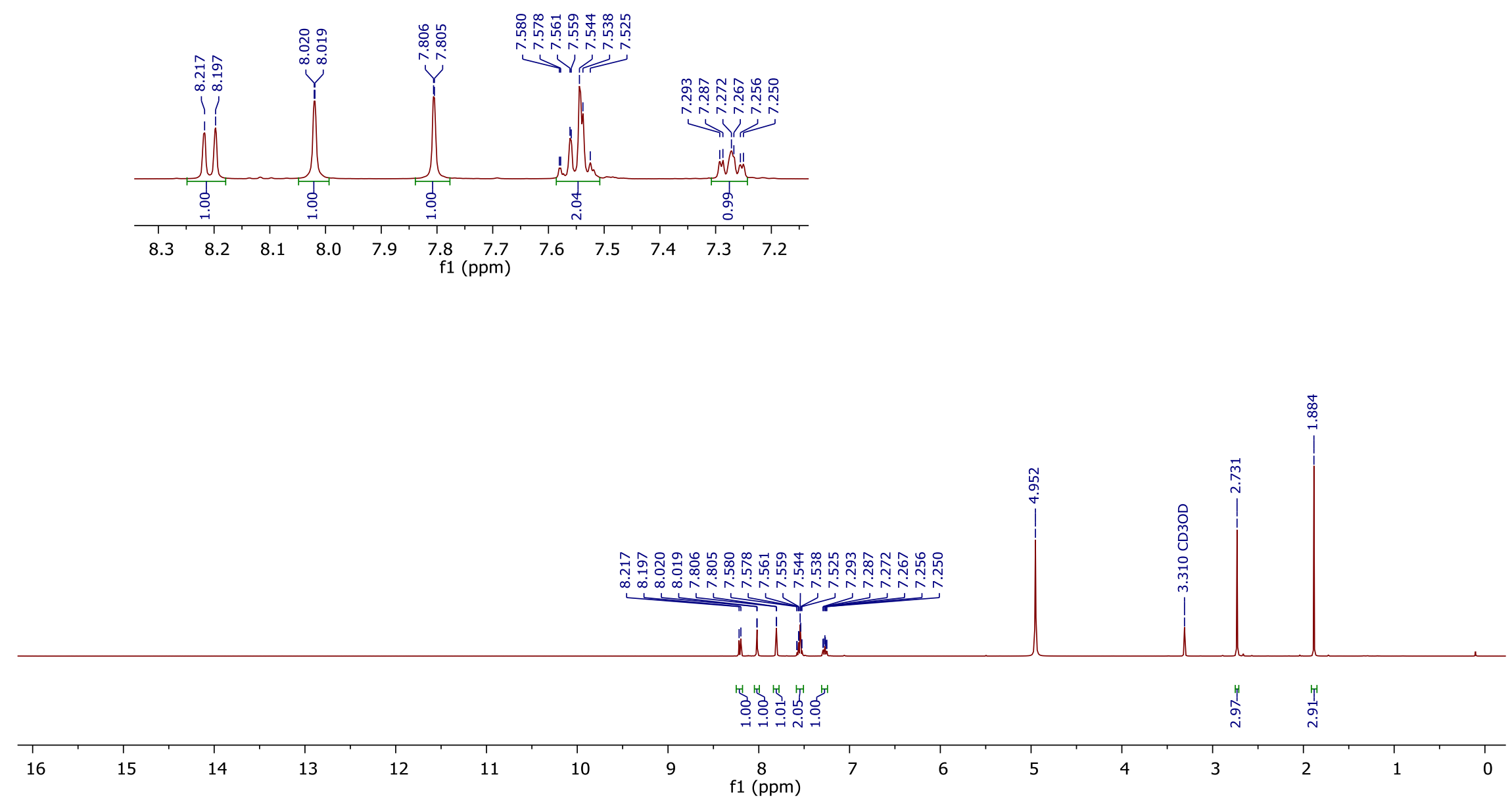

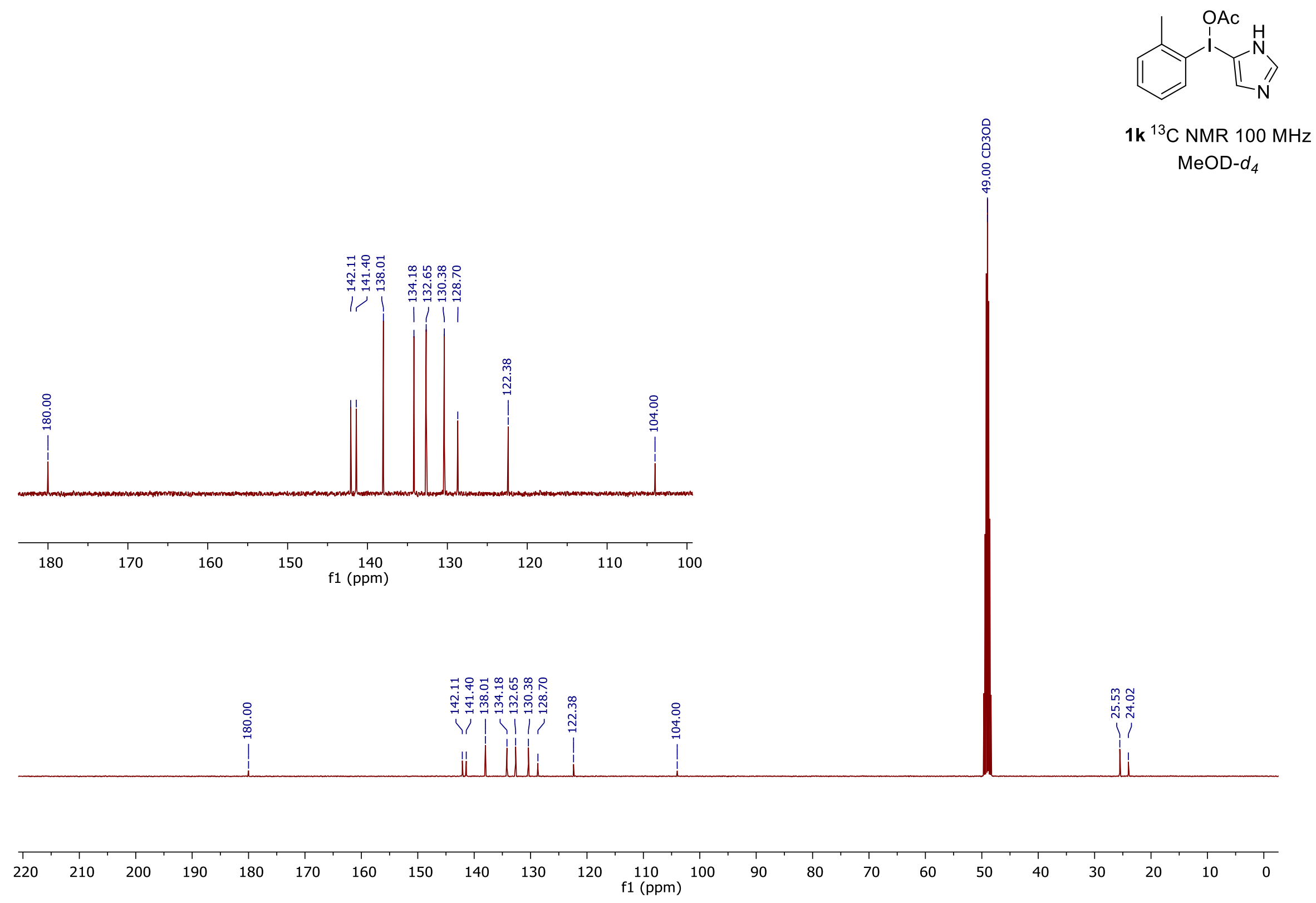


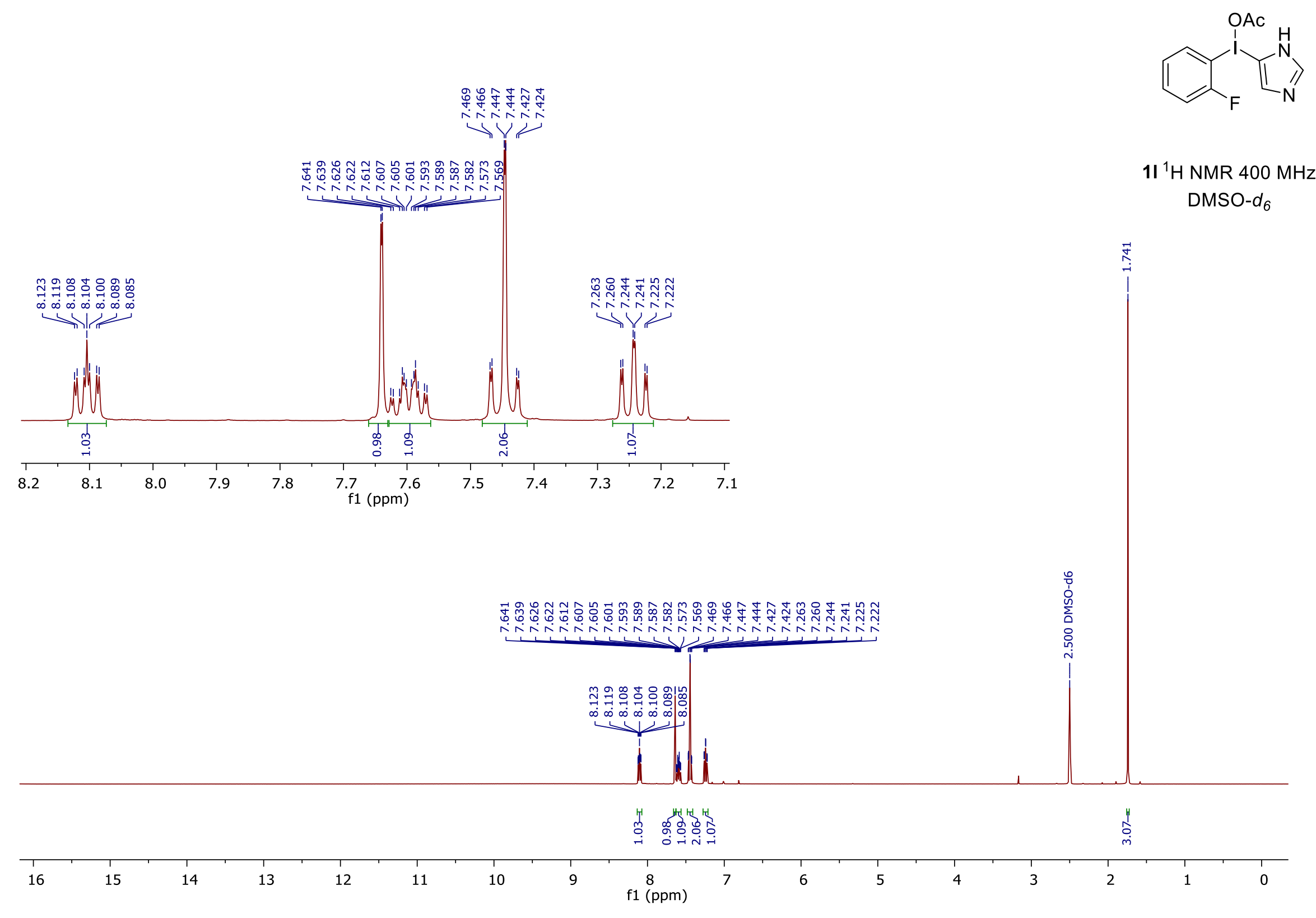




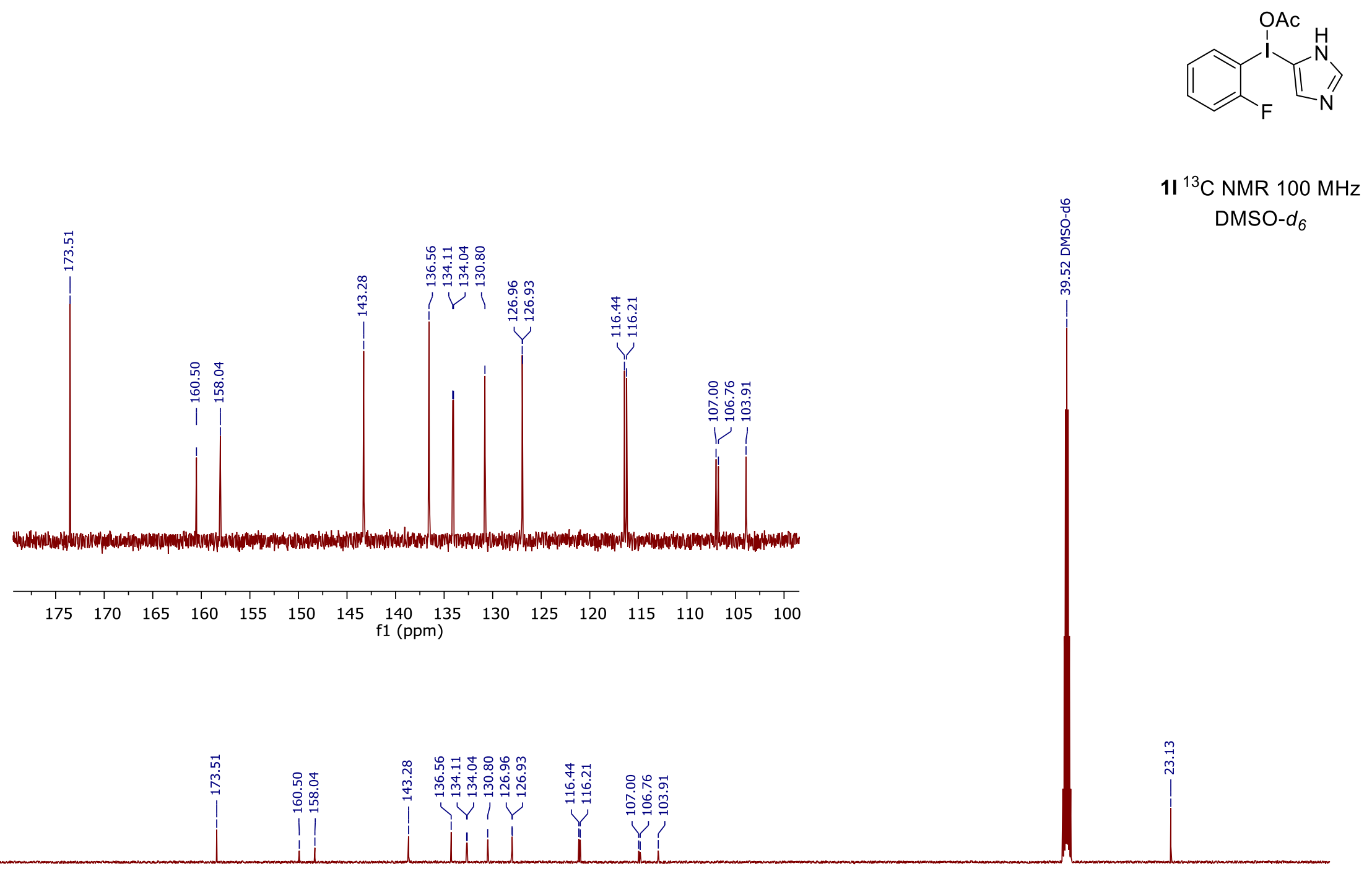

\begin{tabular}{lllllllllllllllllllllll}
\hline & 1 \\
210 & 200 & 190 & 180 & 170 & 160 & 150 & 140 & 130 & 120 & $\begin{array}{c}110 \\
\mathrm{f}(\mathrm{ppm})\end{array}$ & 100 & 90 & 80 & 70 & 60 & 50 & 40 & 30 & 20 & 10 & 0
\end{tabular}




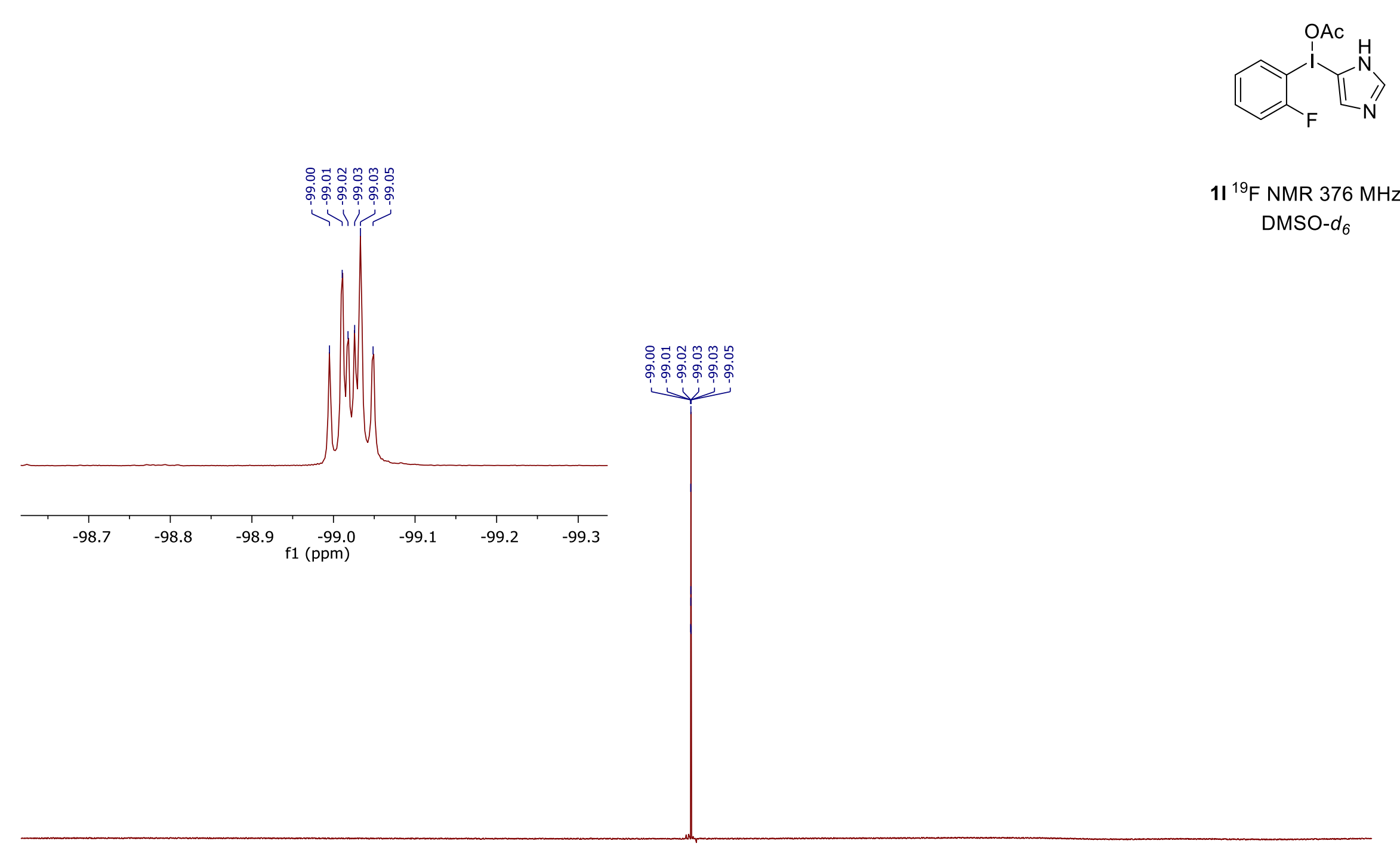

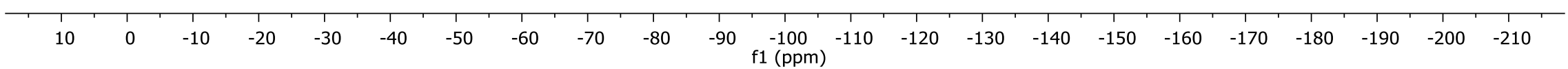




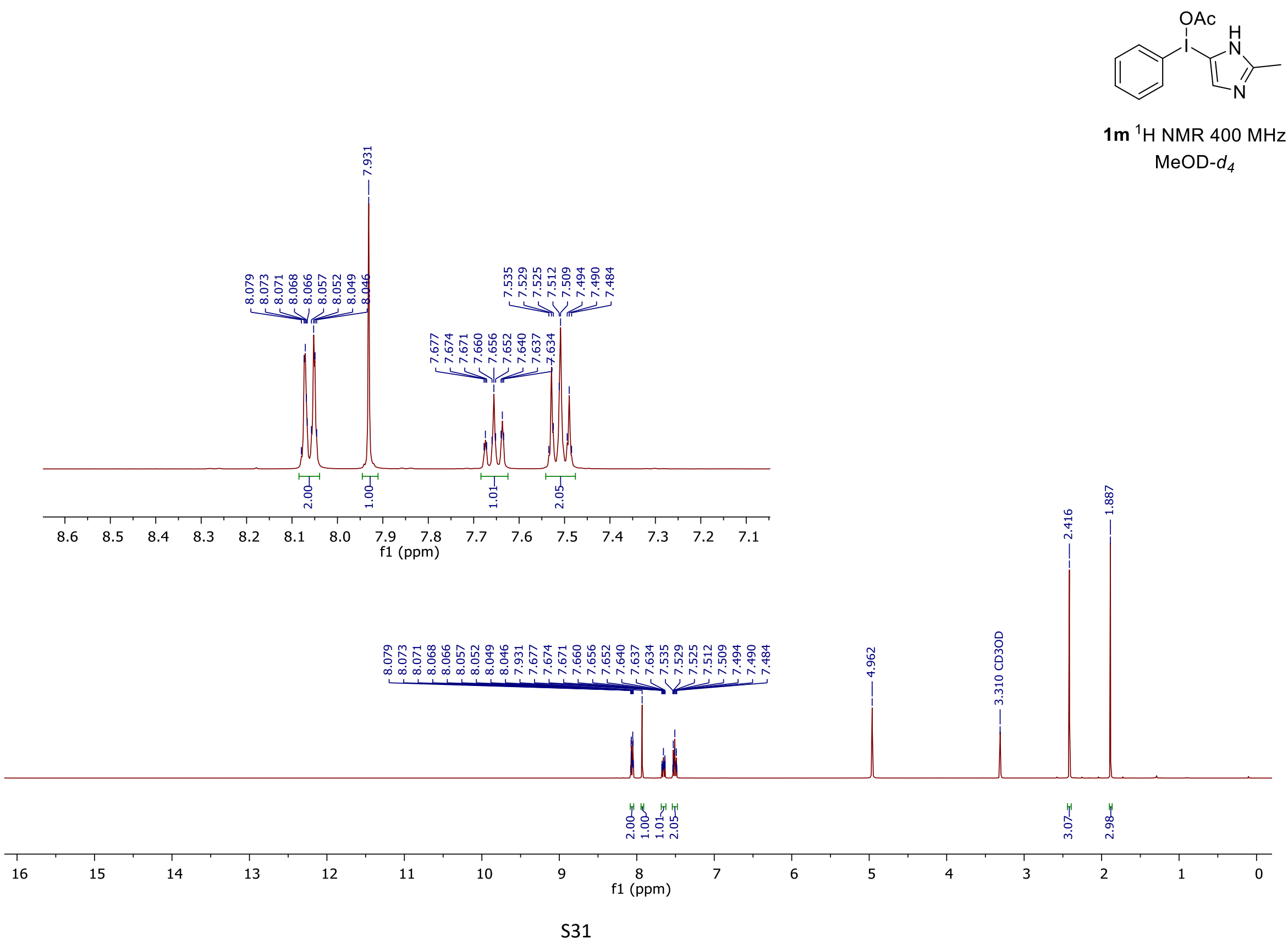



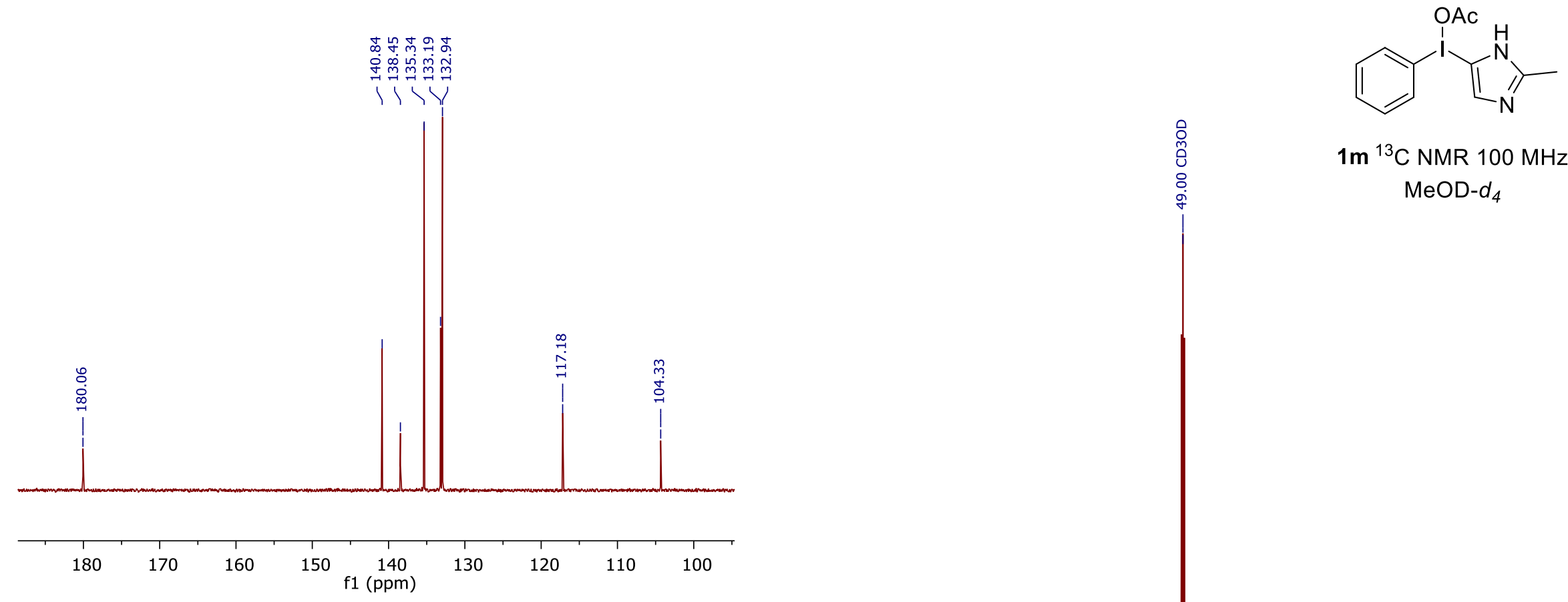

$1 \mathrm{~m}{ }^{13} \mathrm{C}$ NMR $100 \mathrm{MHz}$ MeOD- $d_{4}$
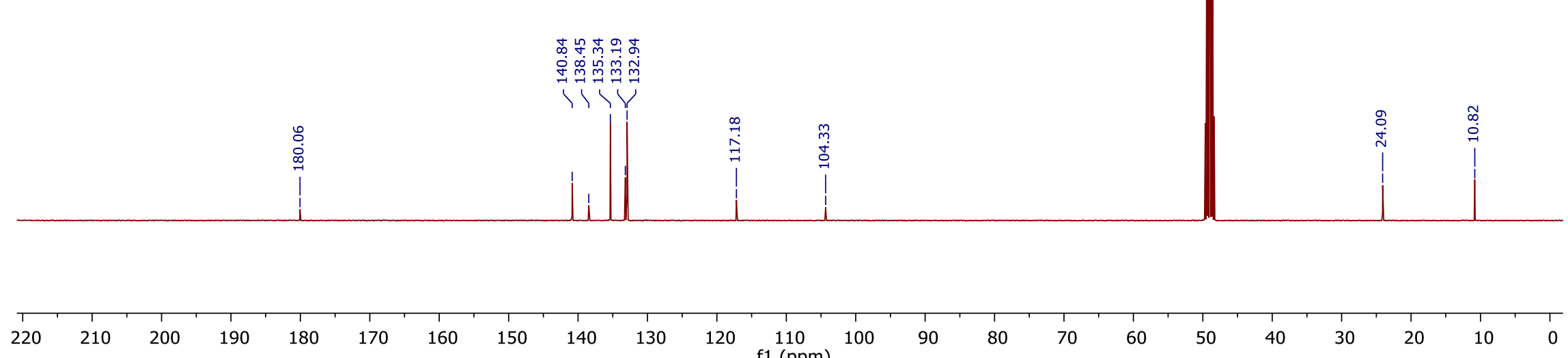

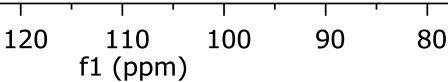

70

$60 \quad 50$ 


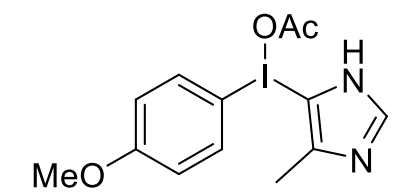

1n ${ }^{1} \mathrm{H}$ NMR $400 \mathrm{MHz}$

MeOD- $d_{4}$
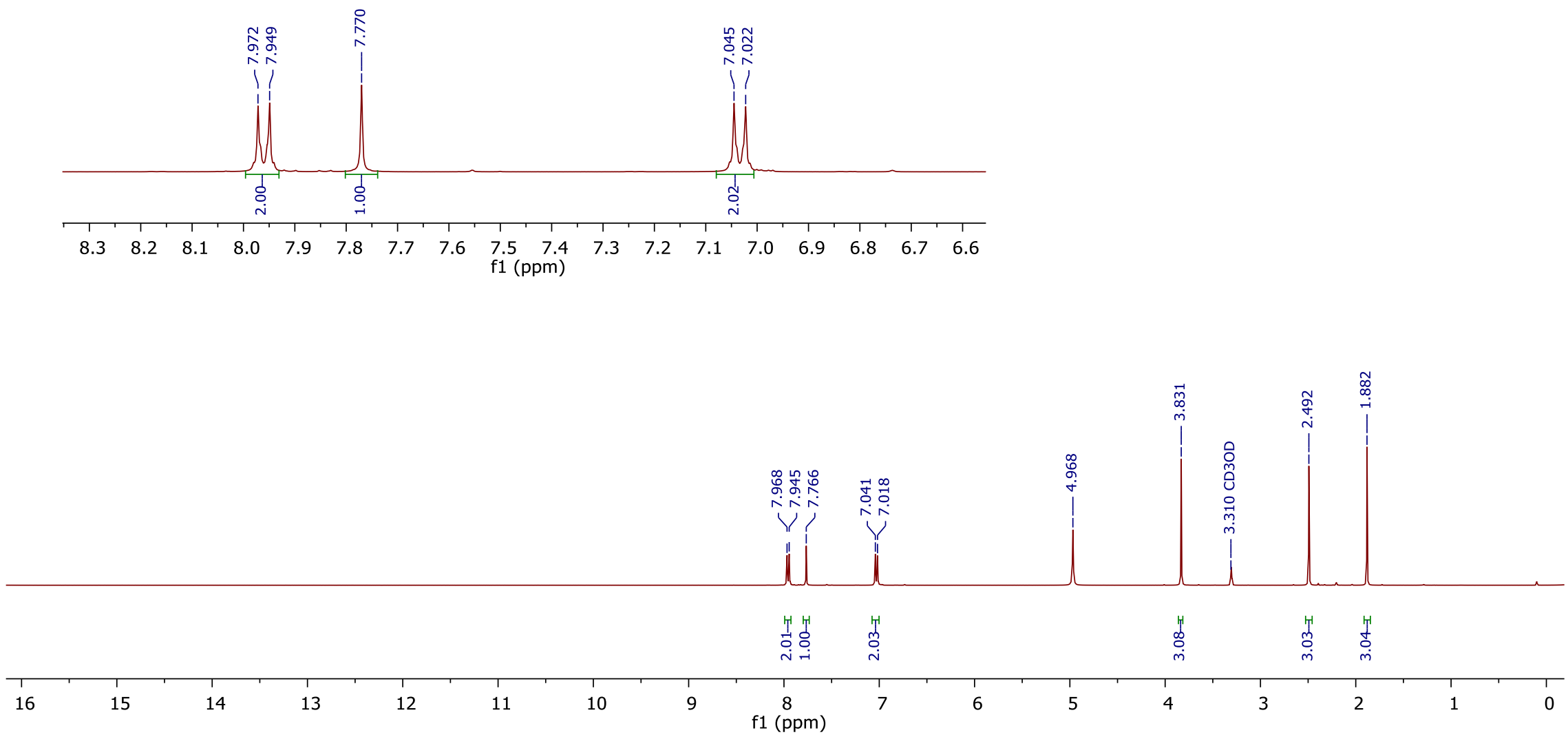

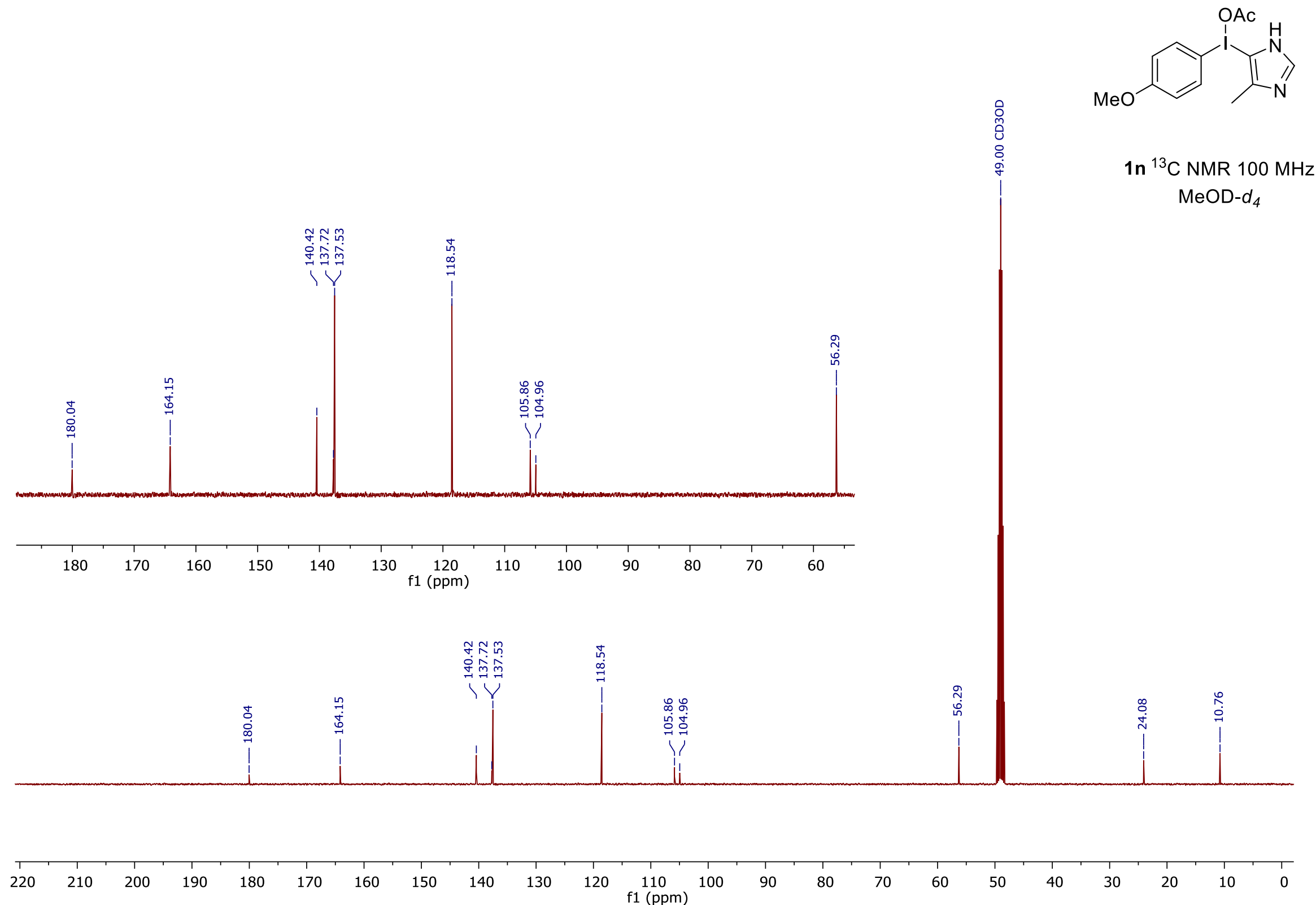


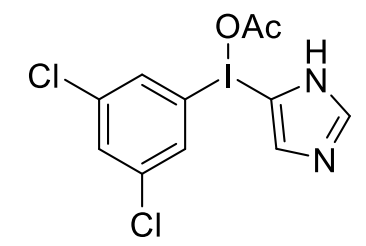

10 ${ }^{1} \mathrm{H}$ NMR $400 \mathrm{MHz}$ MeOD- $d_{4}$
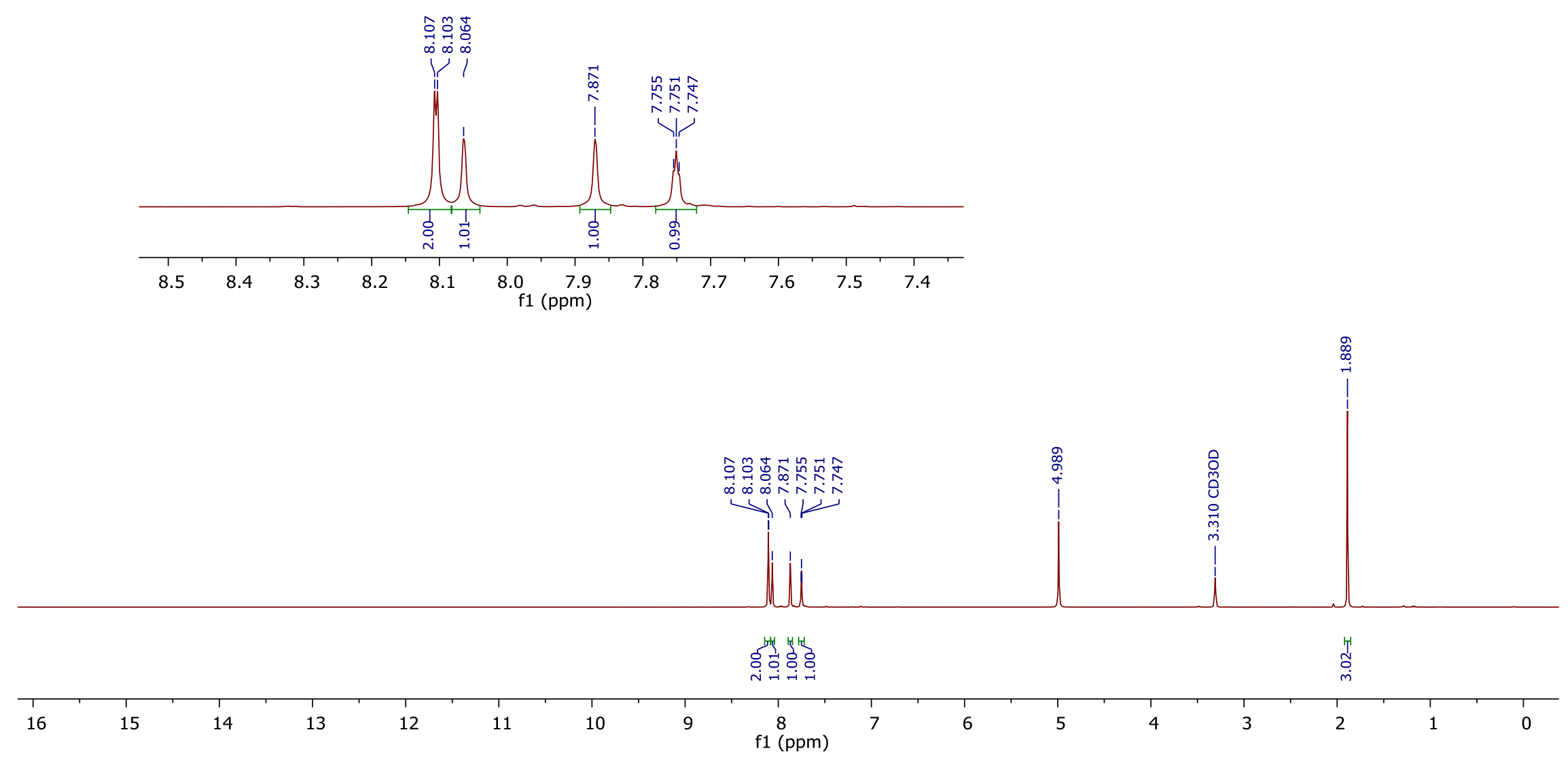

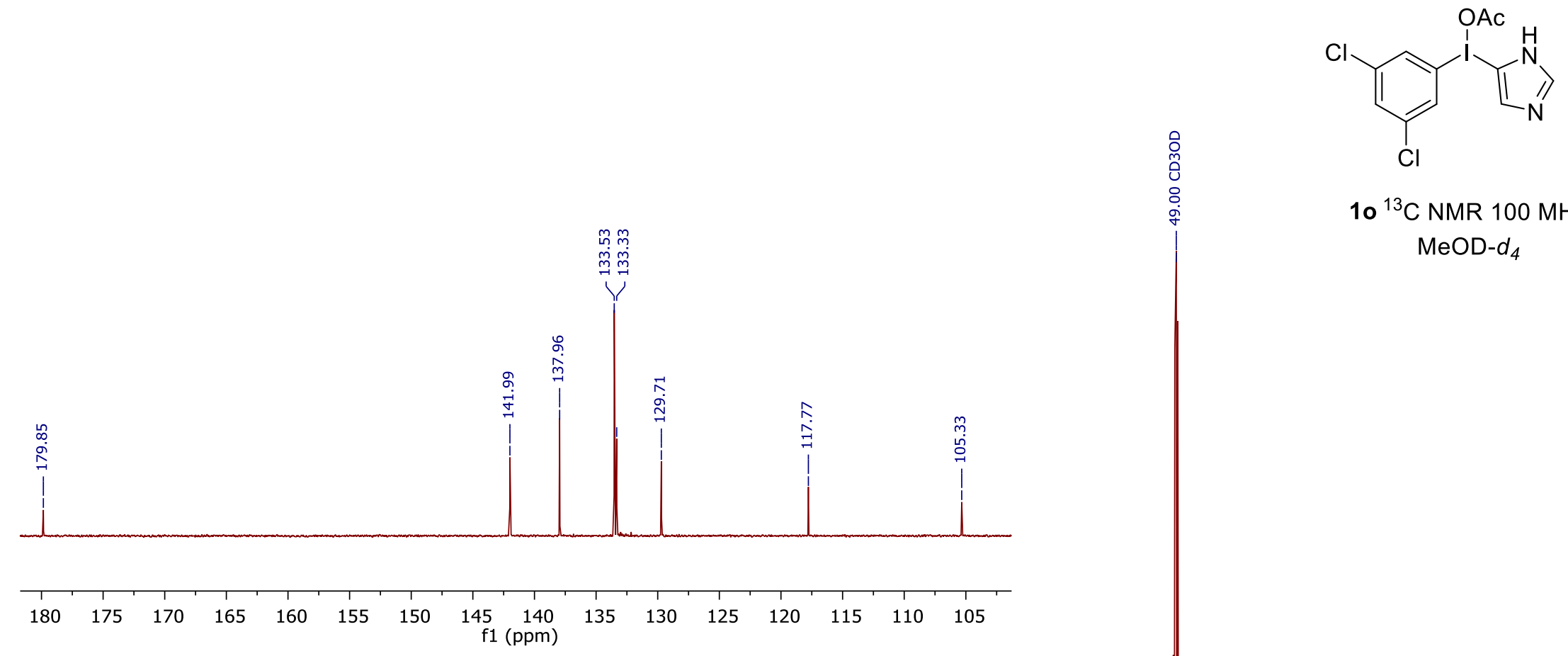

10 ${ }^{13} \mathrm{C}$ NMR $100 \mathrm{MHz}$ MeOD- $d_{4}$

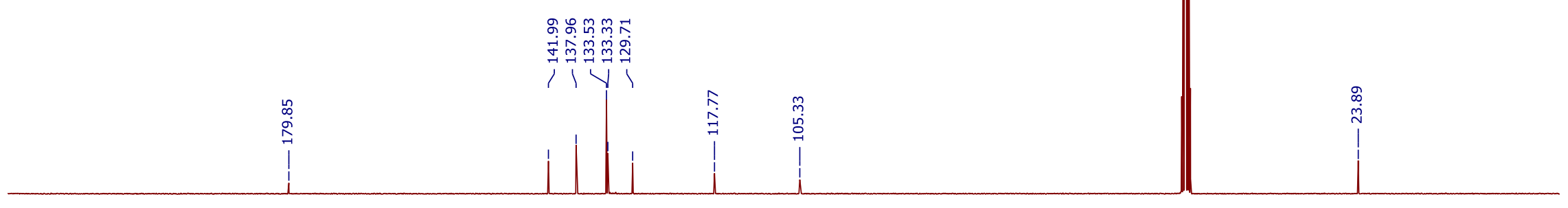

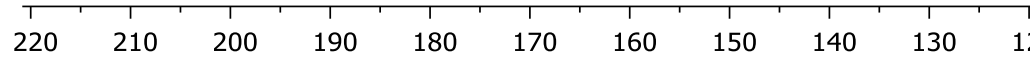
110100

$80 \quad 70 \quad 60 \quad 50$

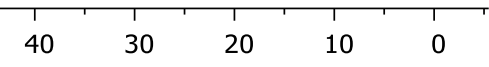




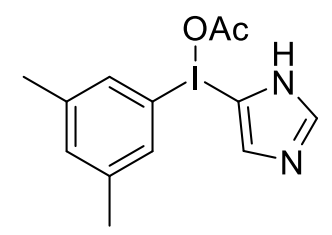

1p ${ }^{1} \mathrm{H}$ NMR $400 \mathrm{MHz}$ MeOD- $d_{4}$
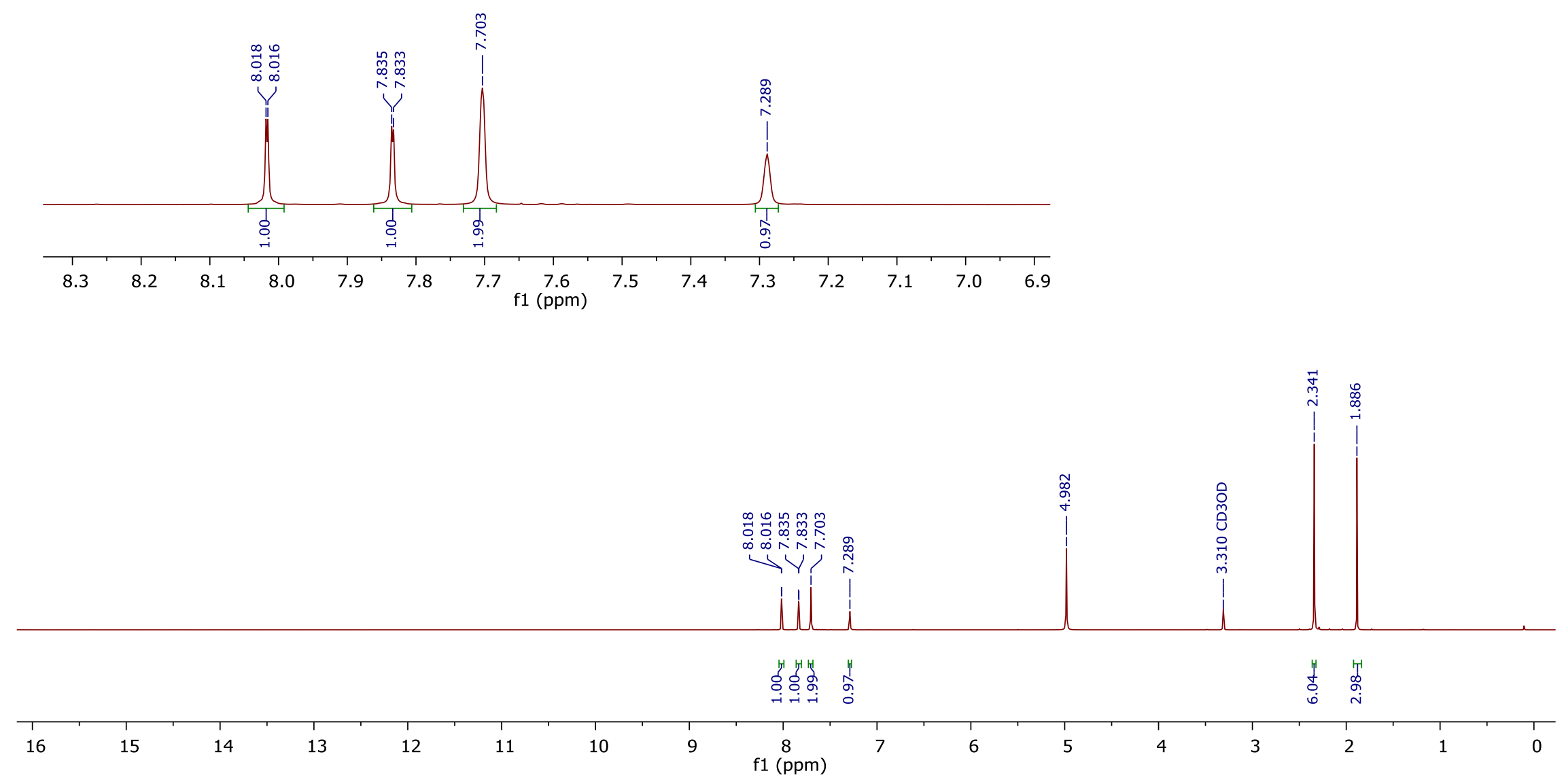


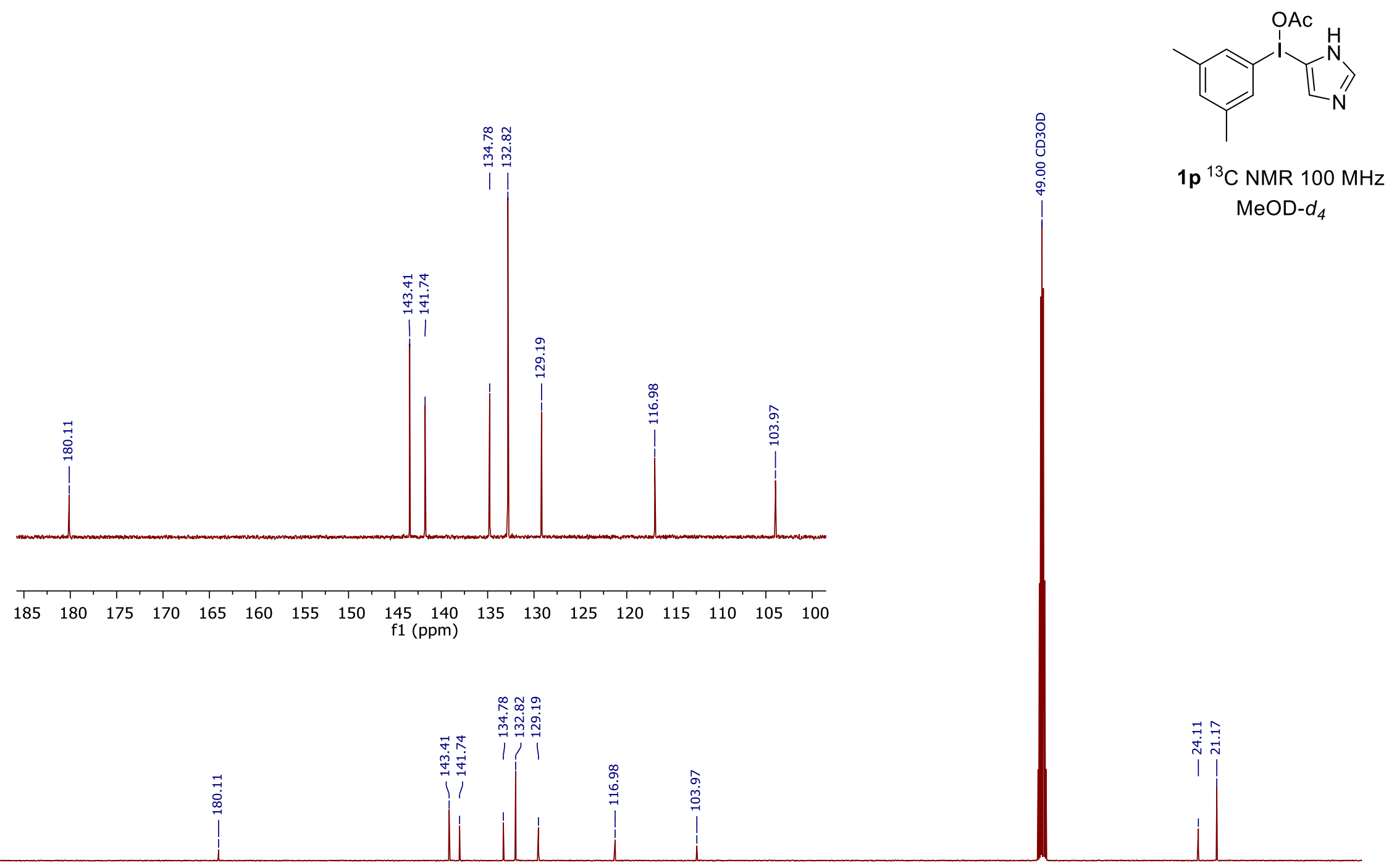

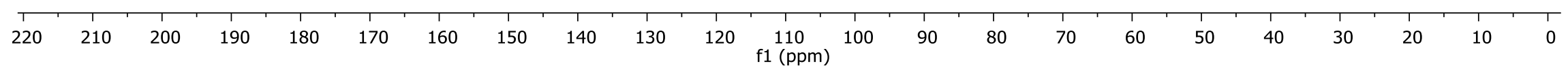




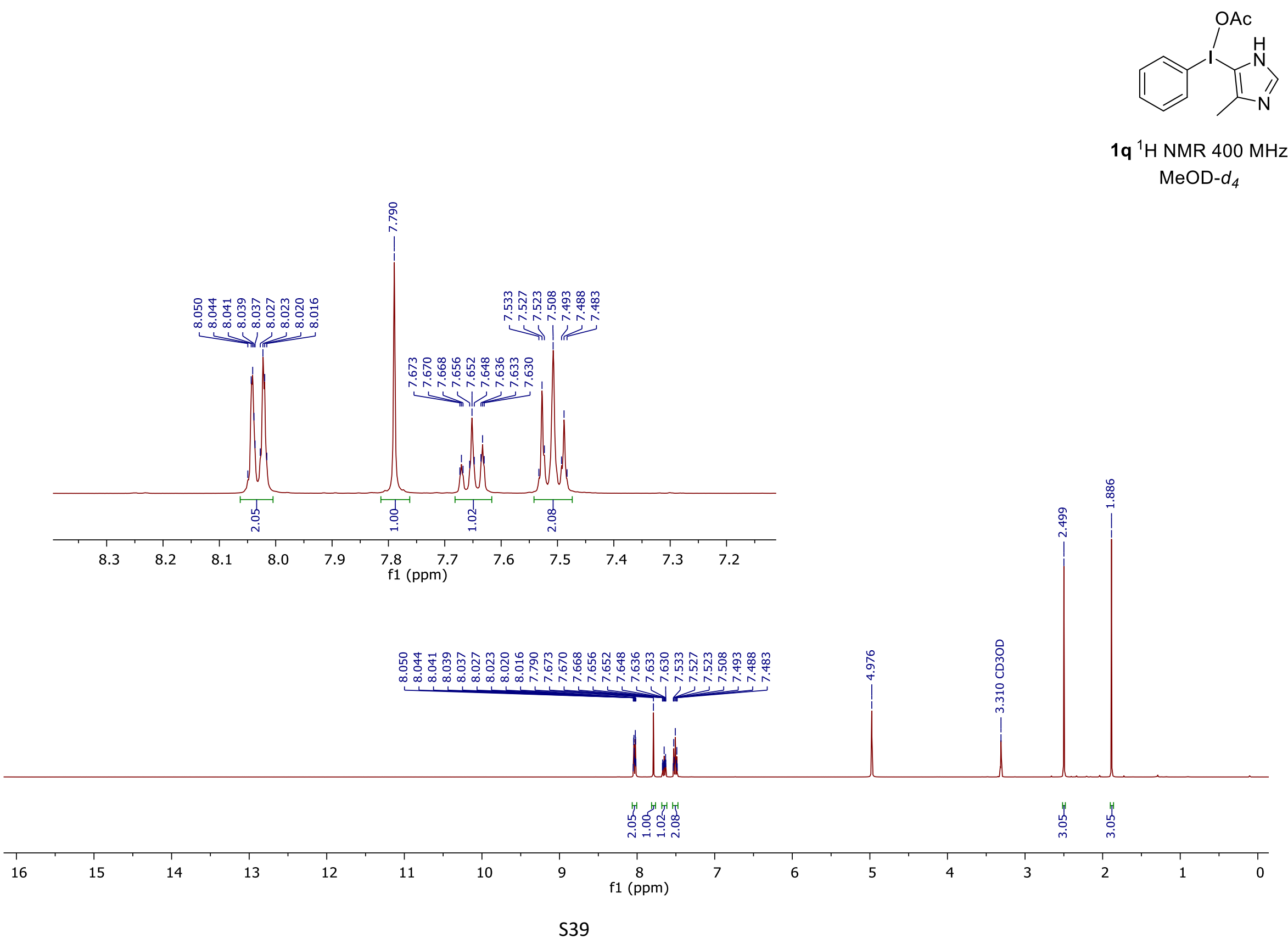



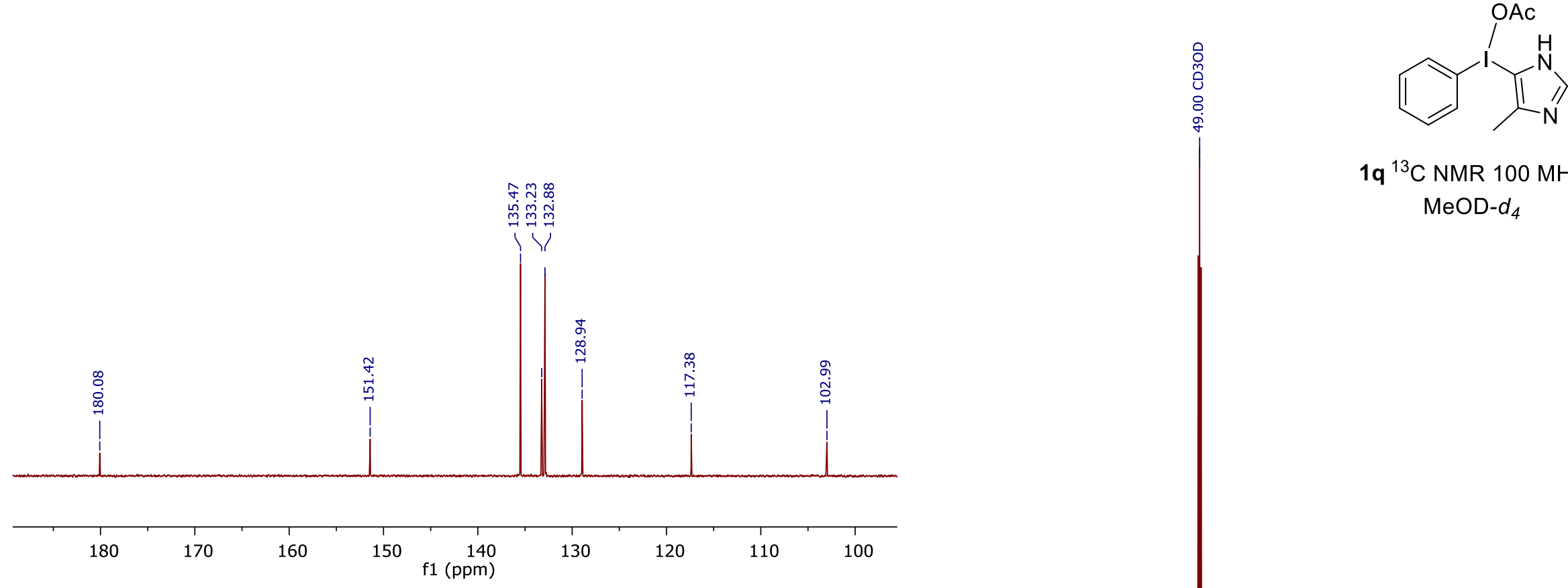

$1 q^{13} \mathrm{C}$ NMR $100 \mathrm{MHz}$ MeOD- $d_{4}$

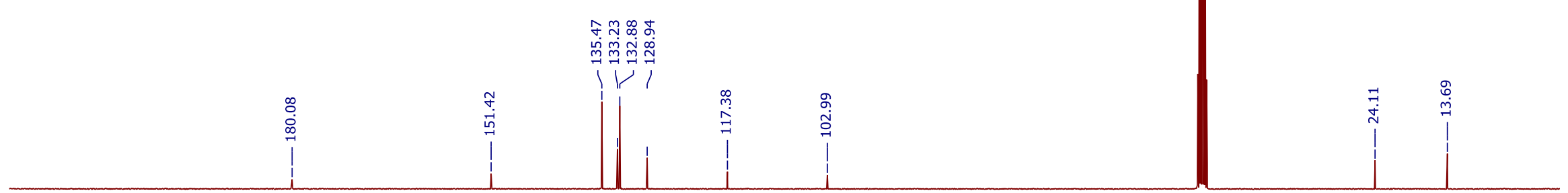

$\begin{array}{llllllllllllllllllllllll}20 & 210 & 200 & 190 & 180 & 170 & 160 & 150 & 140 & 130 & 120 & 110 & 100 & 90 & 80 & 70 & 60 & 50 & 40 & 30 & 20 & 10 & 0\end{array}$ 
2a ${ }^{1} \mathrm{H}$ NMR $400 \mathrm{MHz}$ DMSO- $d_{6}$
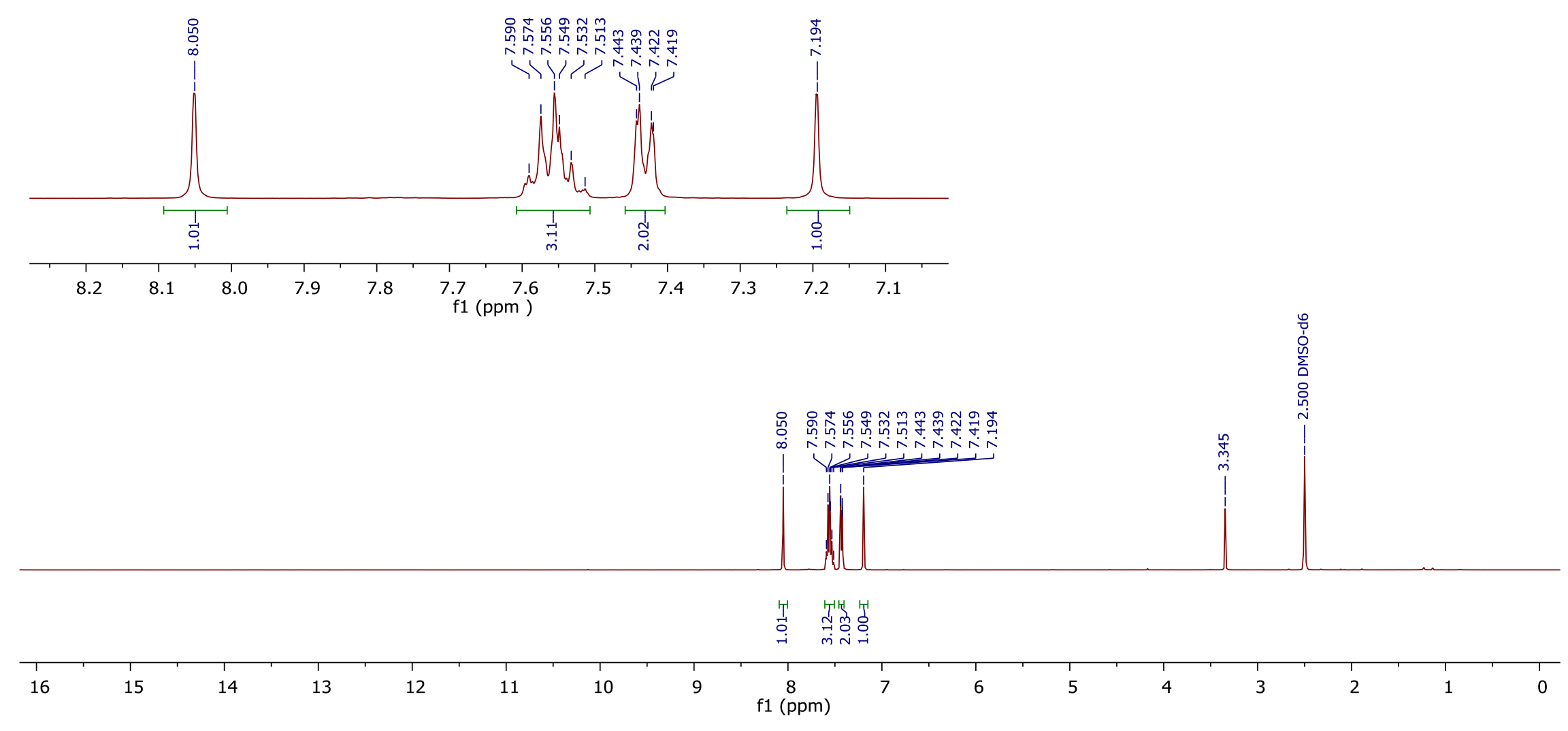


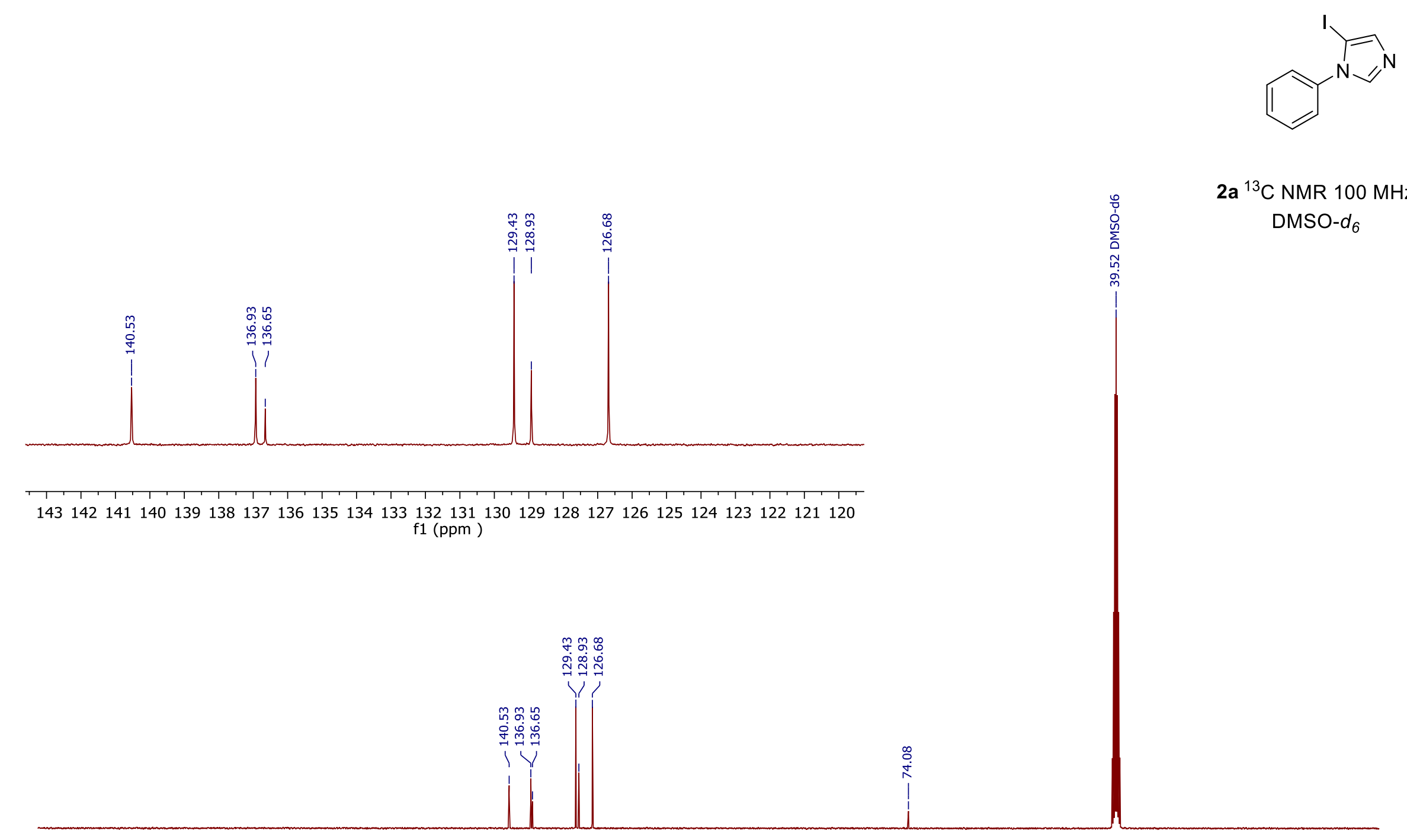

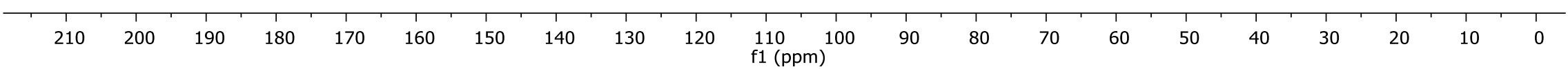




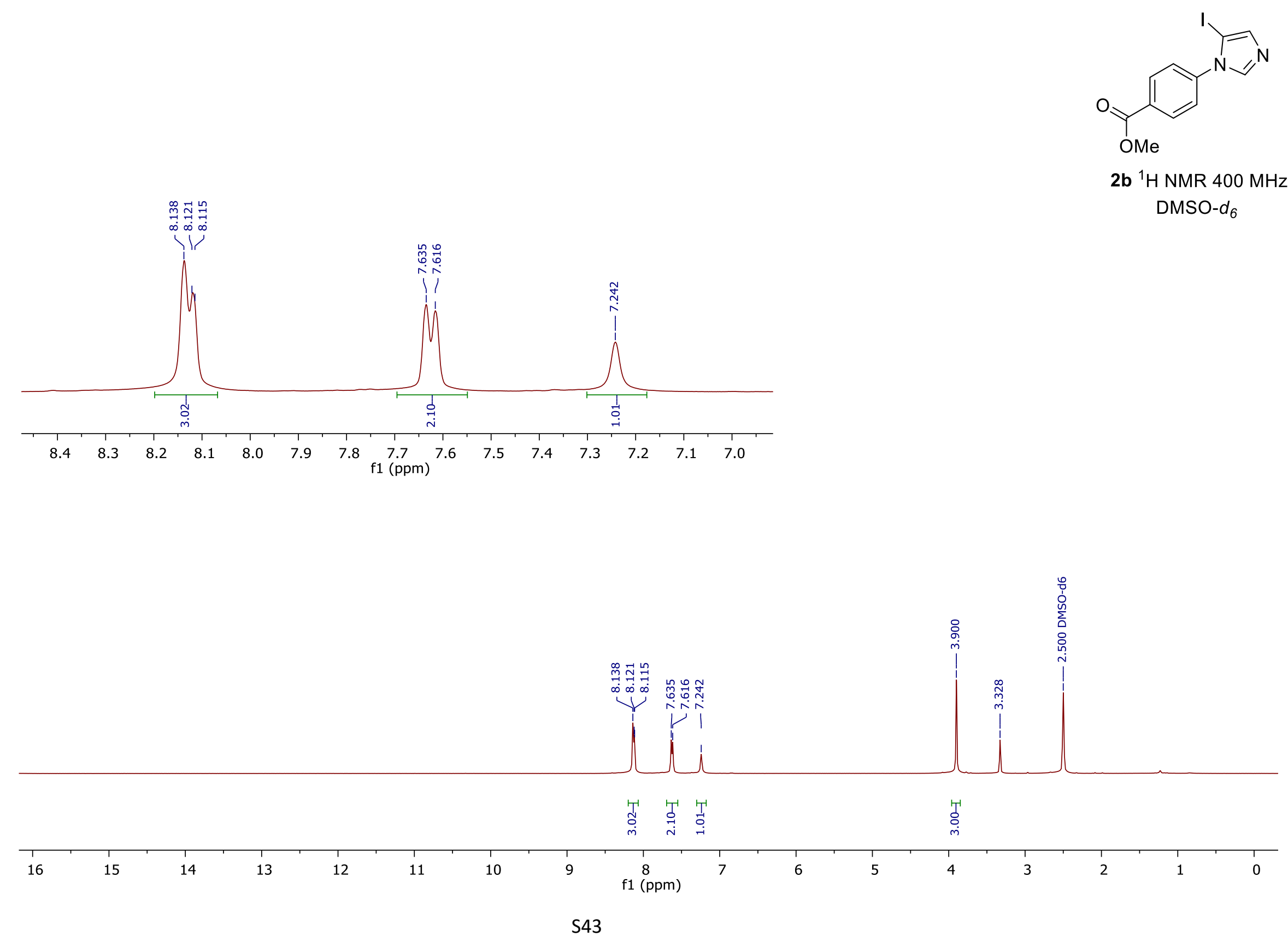



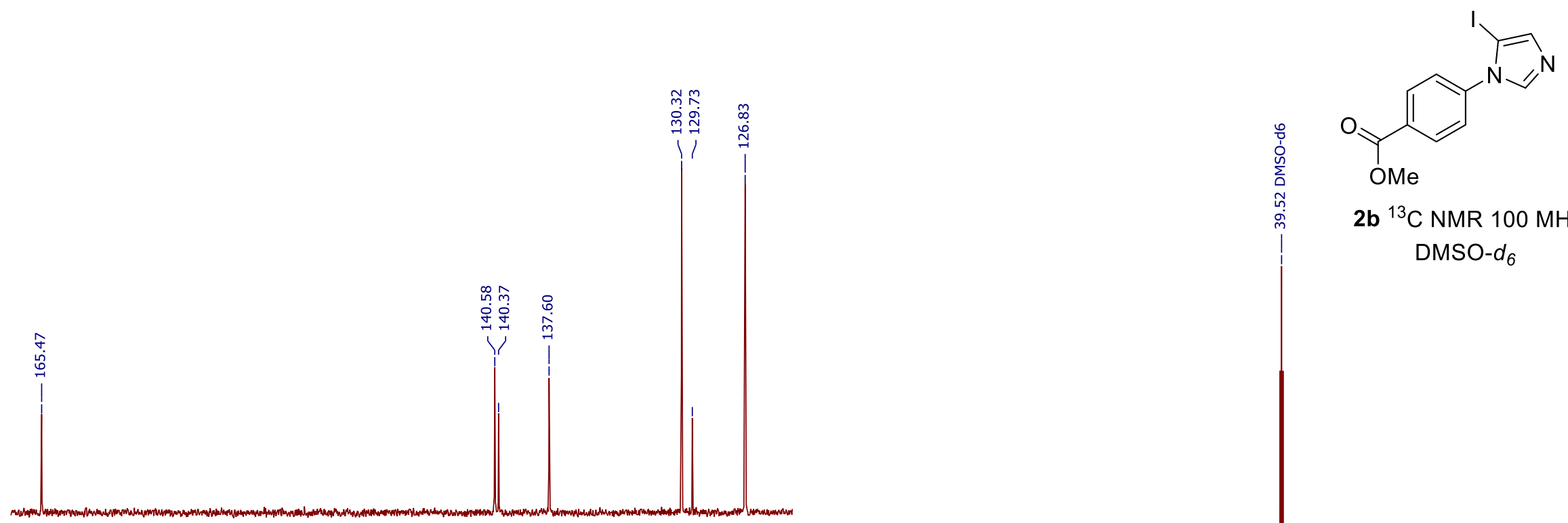

2b ${ }^{13} \mathrm{C}$ NMR $100 \mathrm{MHz}$ DMSO- $d_{6}$
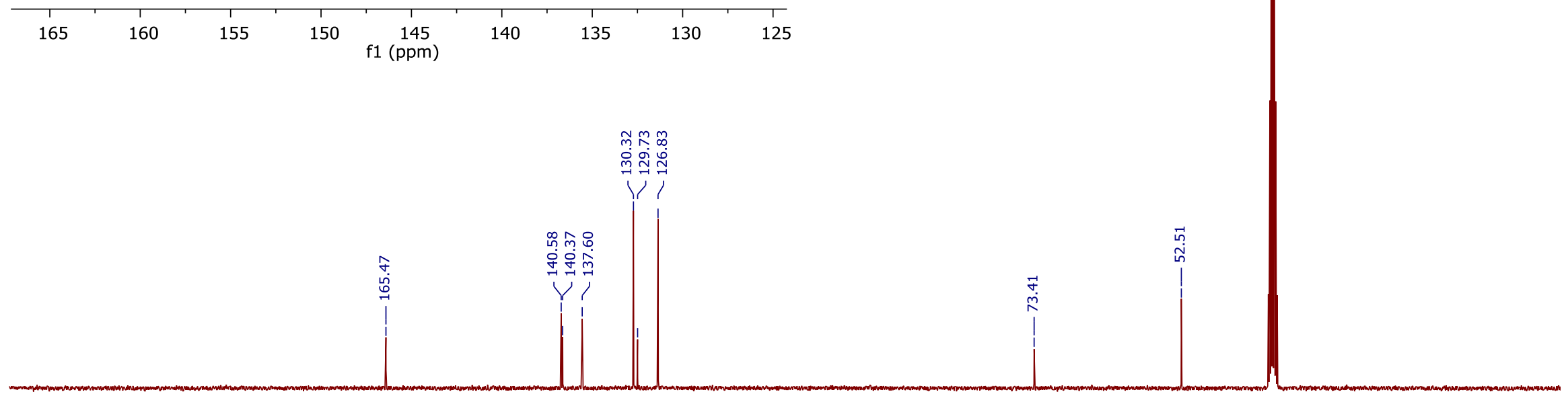


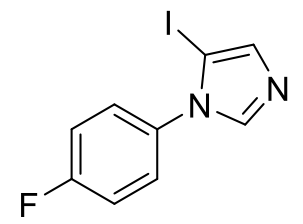

2c ${ }^{1} \mathrm{H}$ NMR $400 \mathrm{MHz}$ $\mathrm{MeOD}-d_{4}$

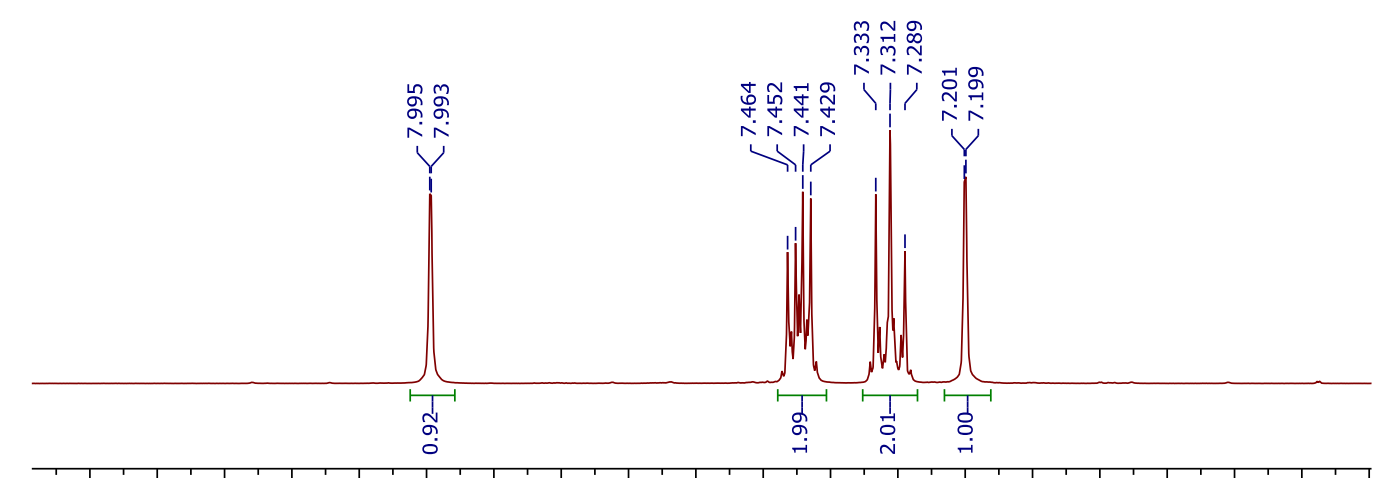

$\begin{array}{llllllllllllllllllll}8.5 & 8.4 & 8.3 & 8.2 & 8.1 & 8.0 & 7.9 & 7.8 & 7.7 & 7.6 & 7.5 & 7.4 & 7.3 & 7.2 & 7.1 & 7.0 & 6.9 & 6.8 & 6.7 & 6.6\end{array}$ $\mathrm{f} 1$ (ppm)

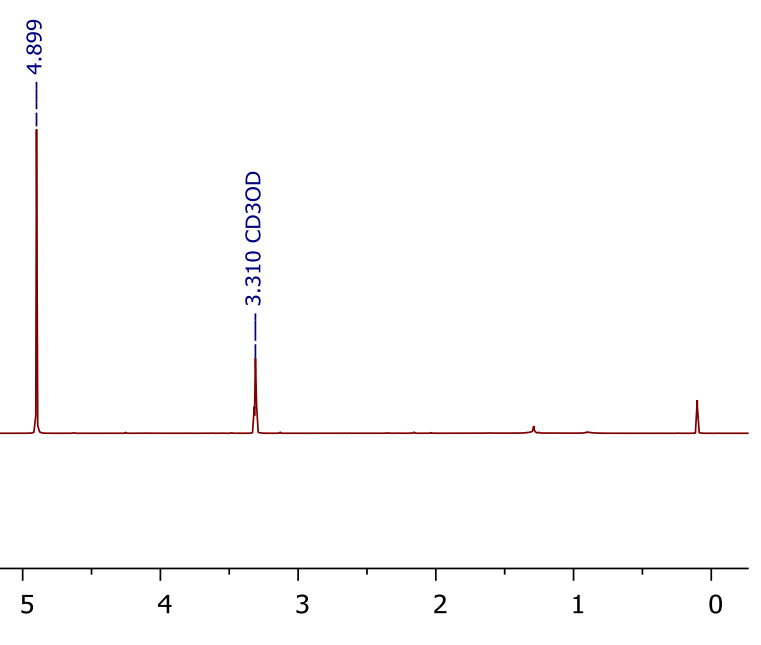



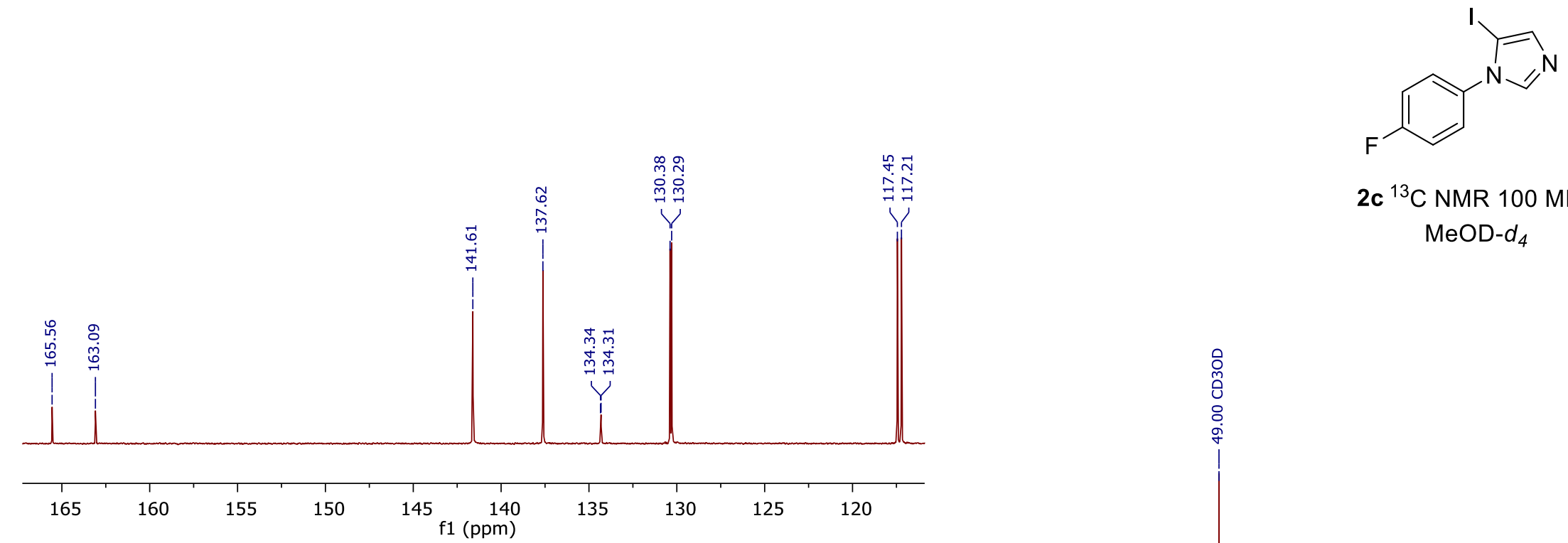

2c ${ }^{13} \mathrm{C}$ NMR $100 \mathrm{MHz}$ $\mathrm{MeOD}-d_{4}$

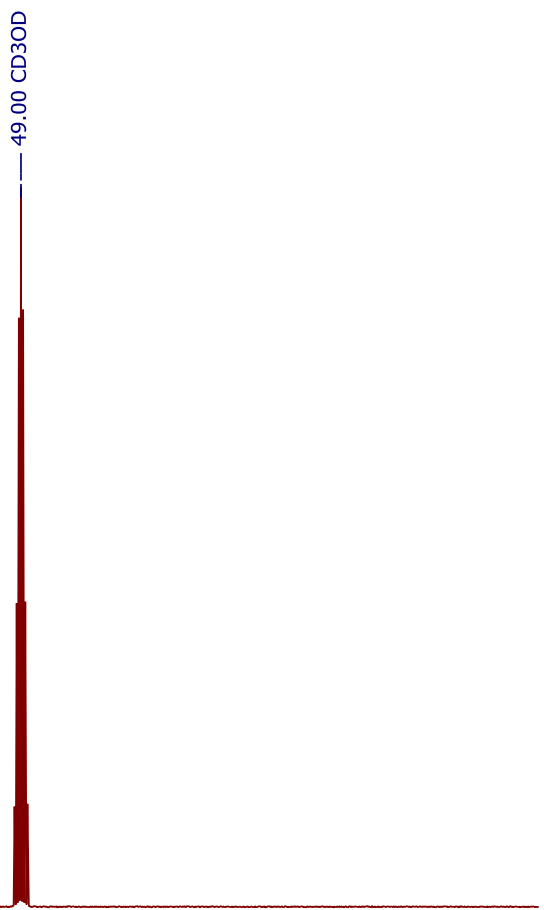




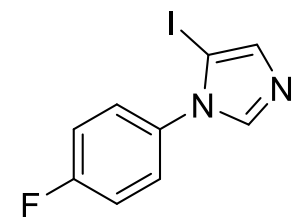

2c ${ }^{19} \mathrm{~F}$ NMR $376 \mathrm{MHz}$ $\mathrm{MeOD}-d_{4}$
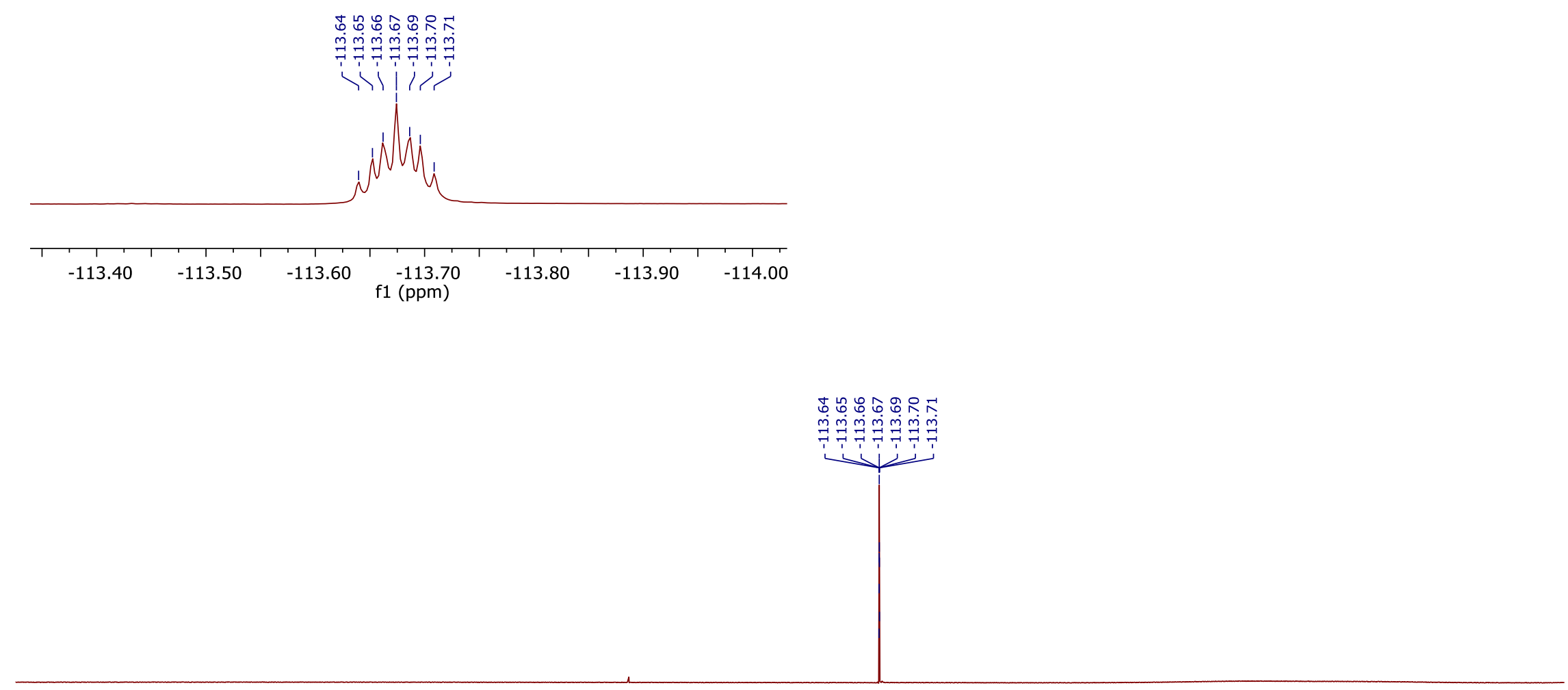

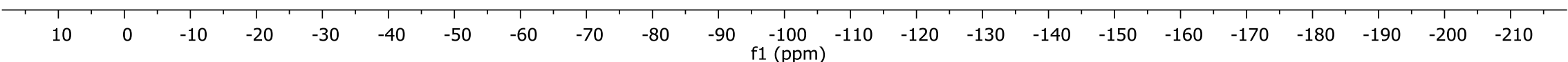




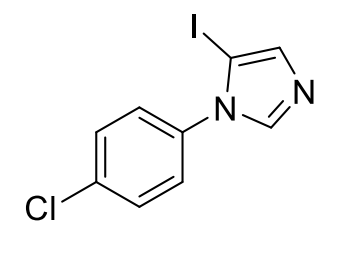

2d ${ }^{1} \mathrm{H}$ NMR $400 \mathrm{MHz}$ DMSO- $d_{6}$
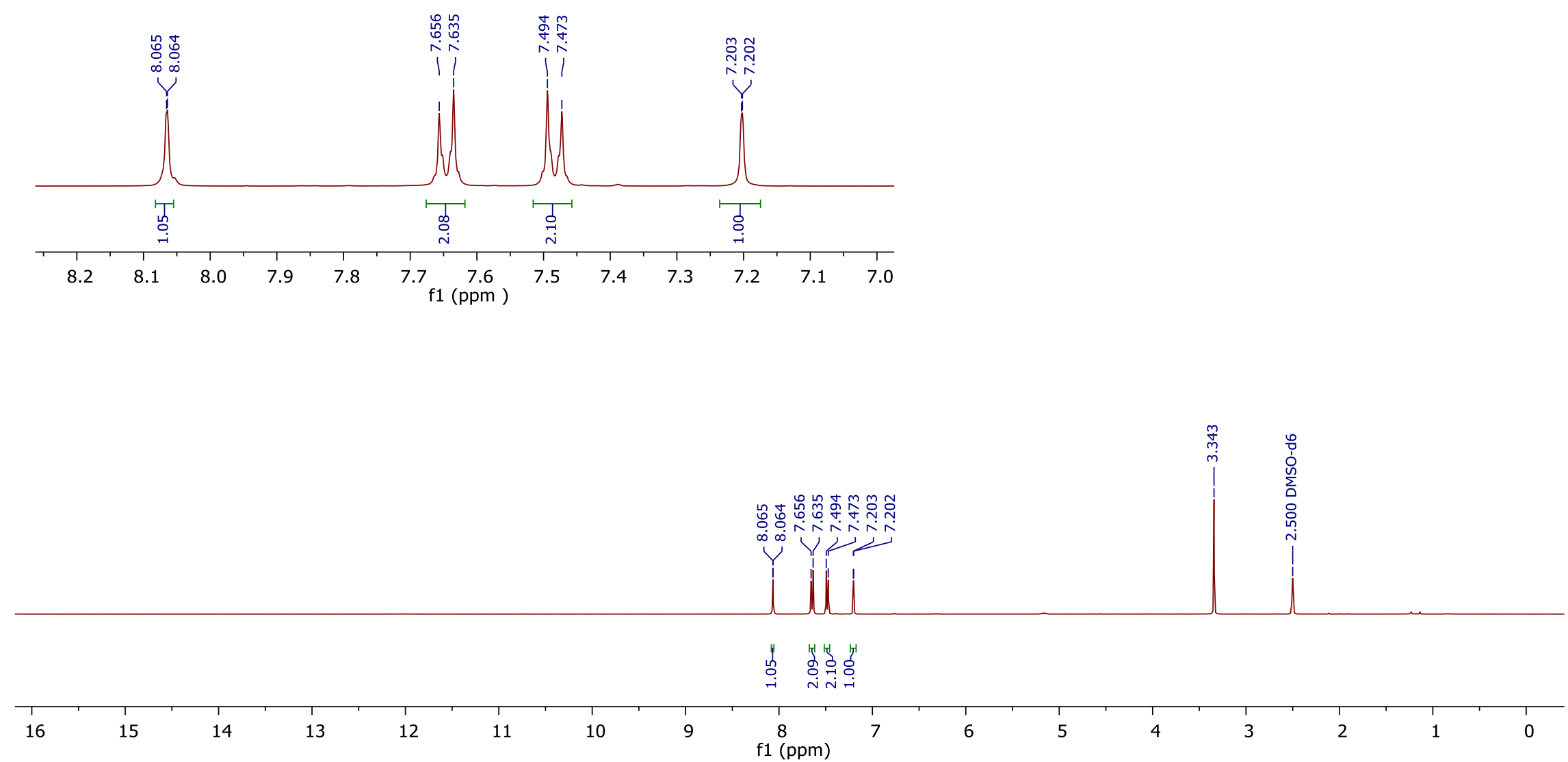


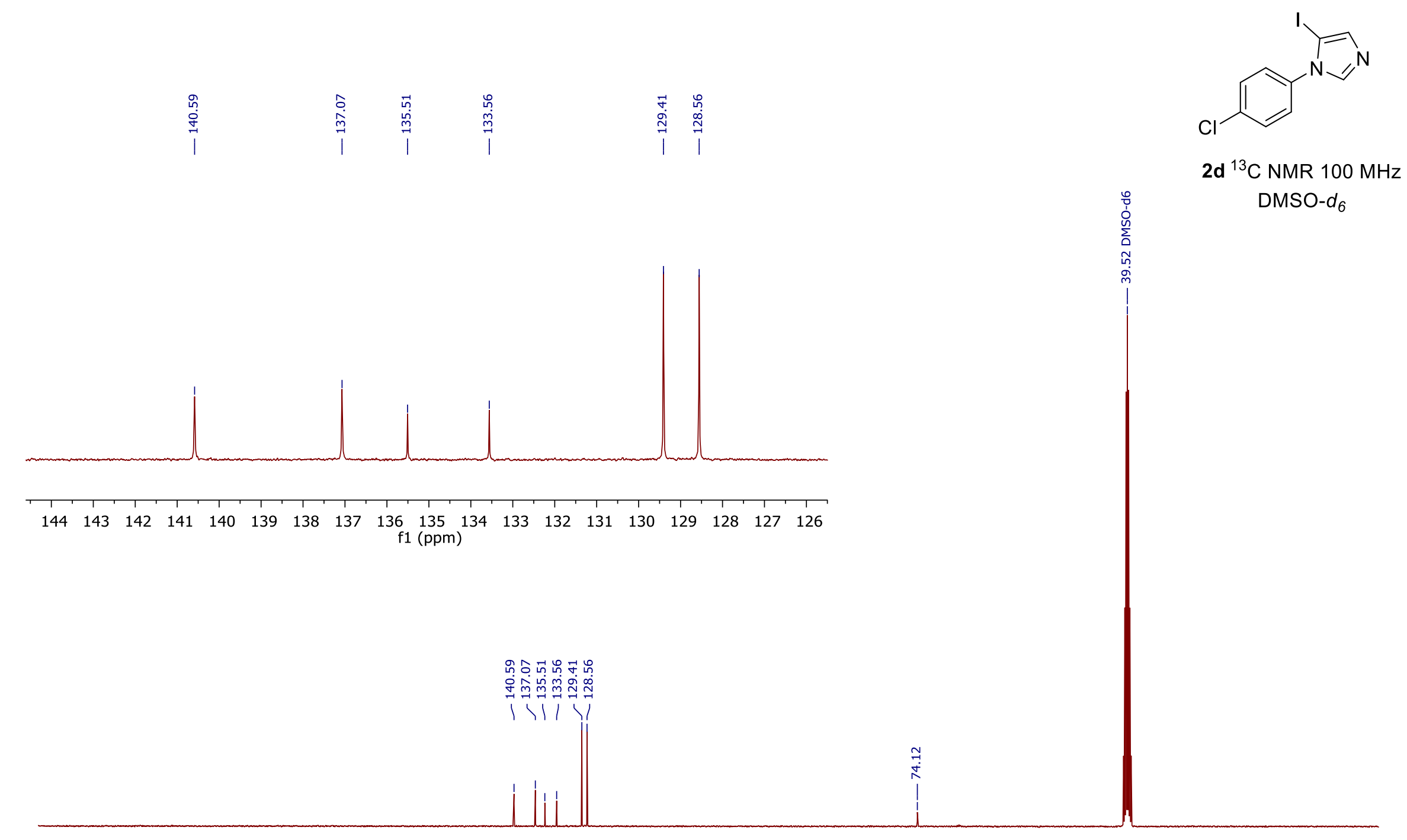

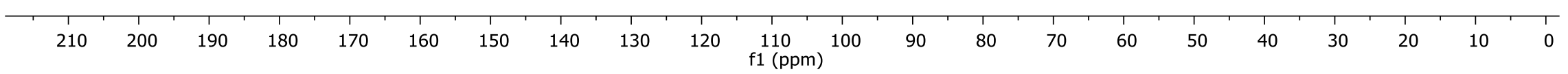




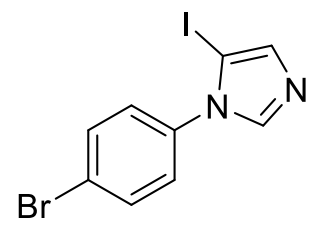

2e ${ }^{1} \mathrm{H}$ NMR $400 \mathrm{MHz}$ MeOD- $d_{4}$
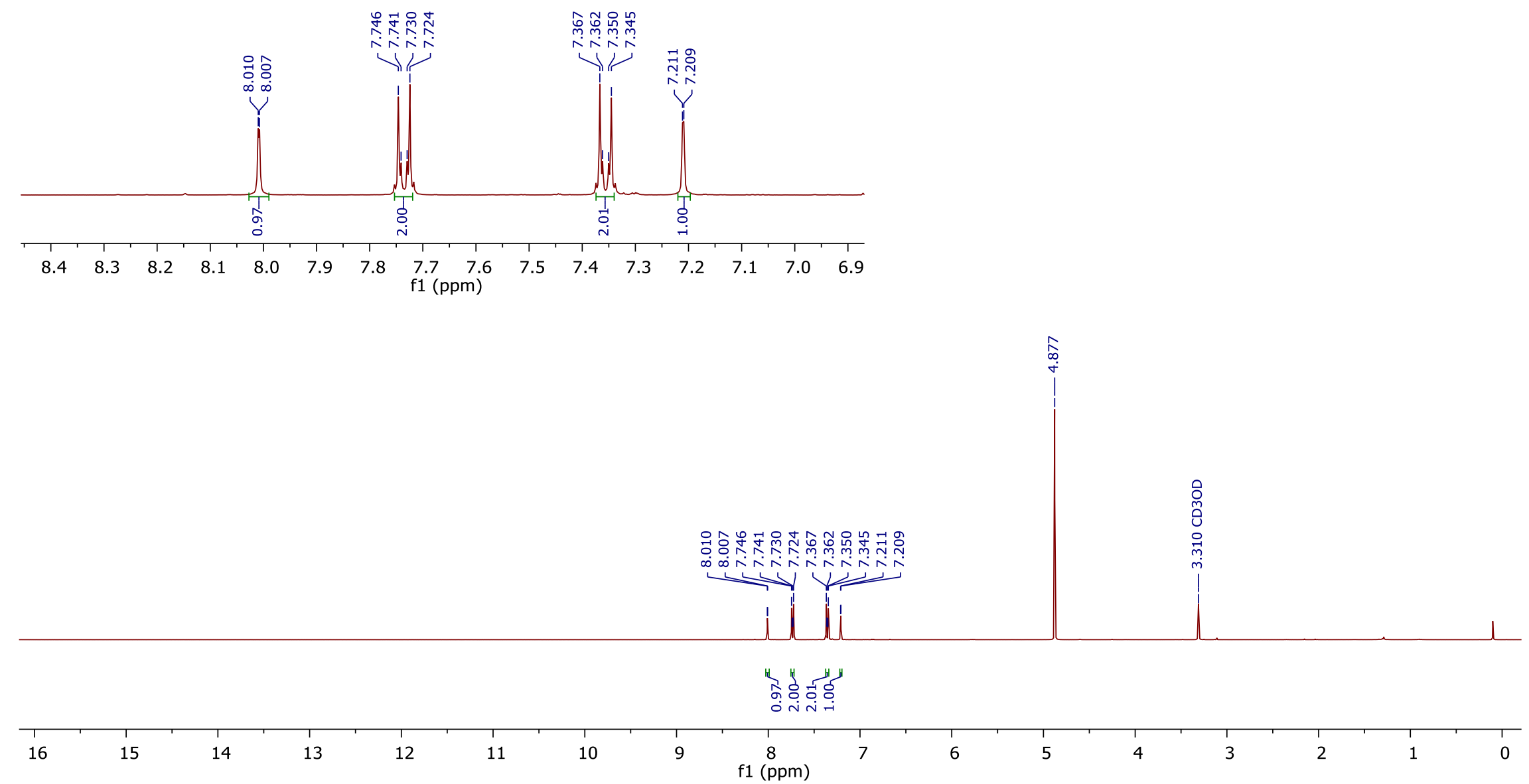


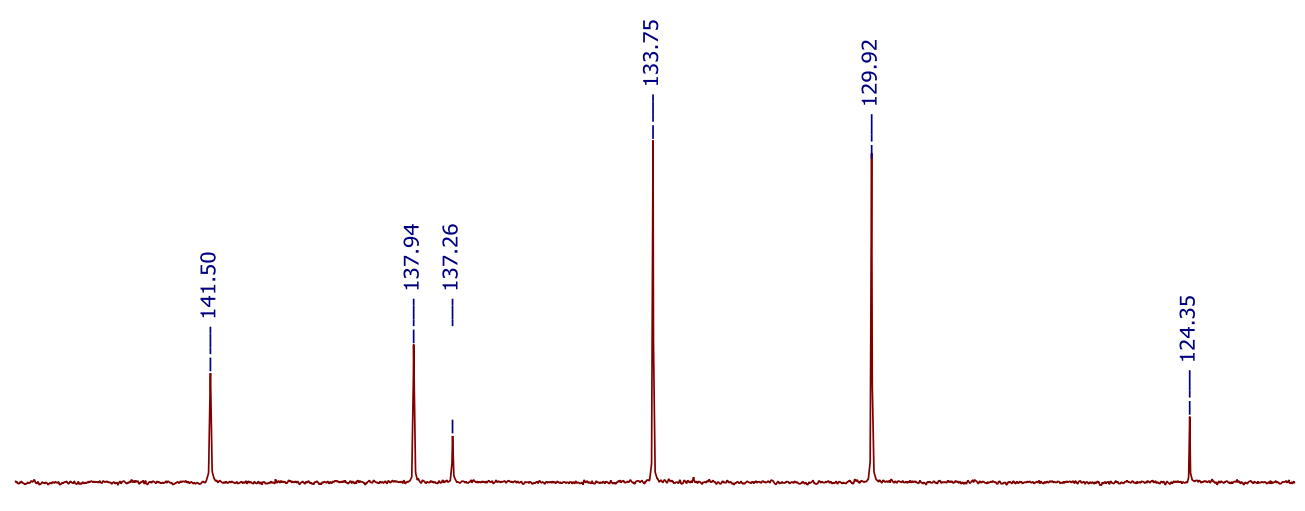

144143142141140139138137136135134133132131130129128127126125124123 f1 (ppm)

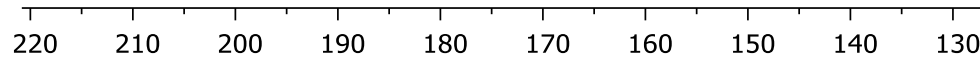

\section{$120 \quad 110$}

$100 \quad 90$

80

70

60

50

40

30

20 


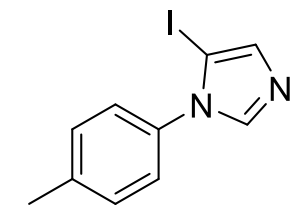

2f ${ }^{1} \mathrm{H}$ NMR $400 \mathrm{MHz}$ DMSO- $d_{6}$
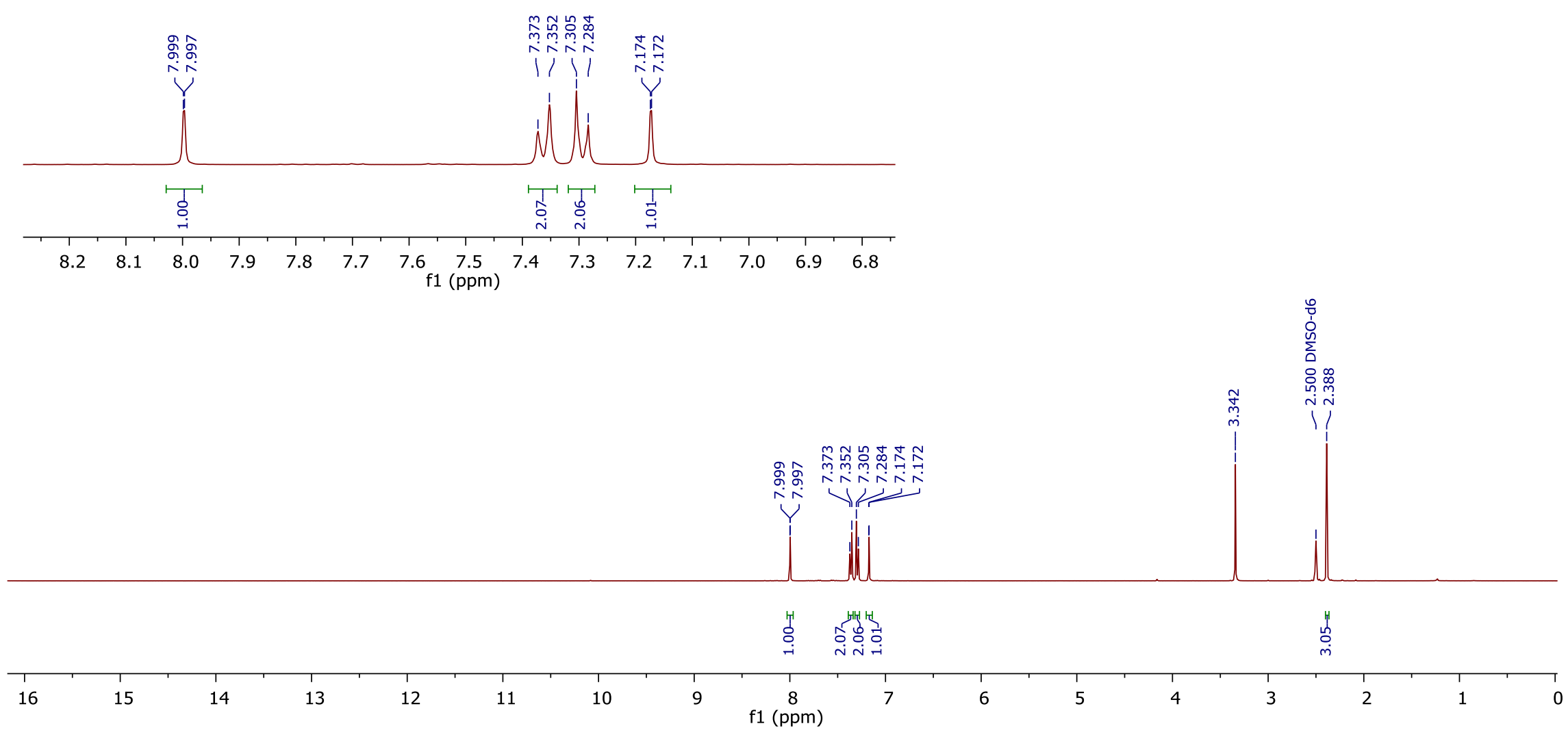


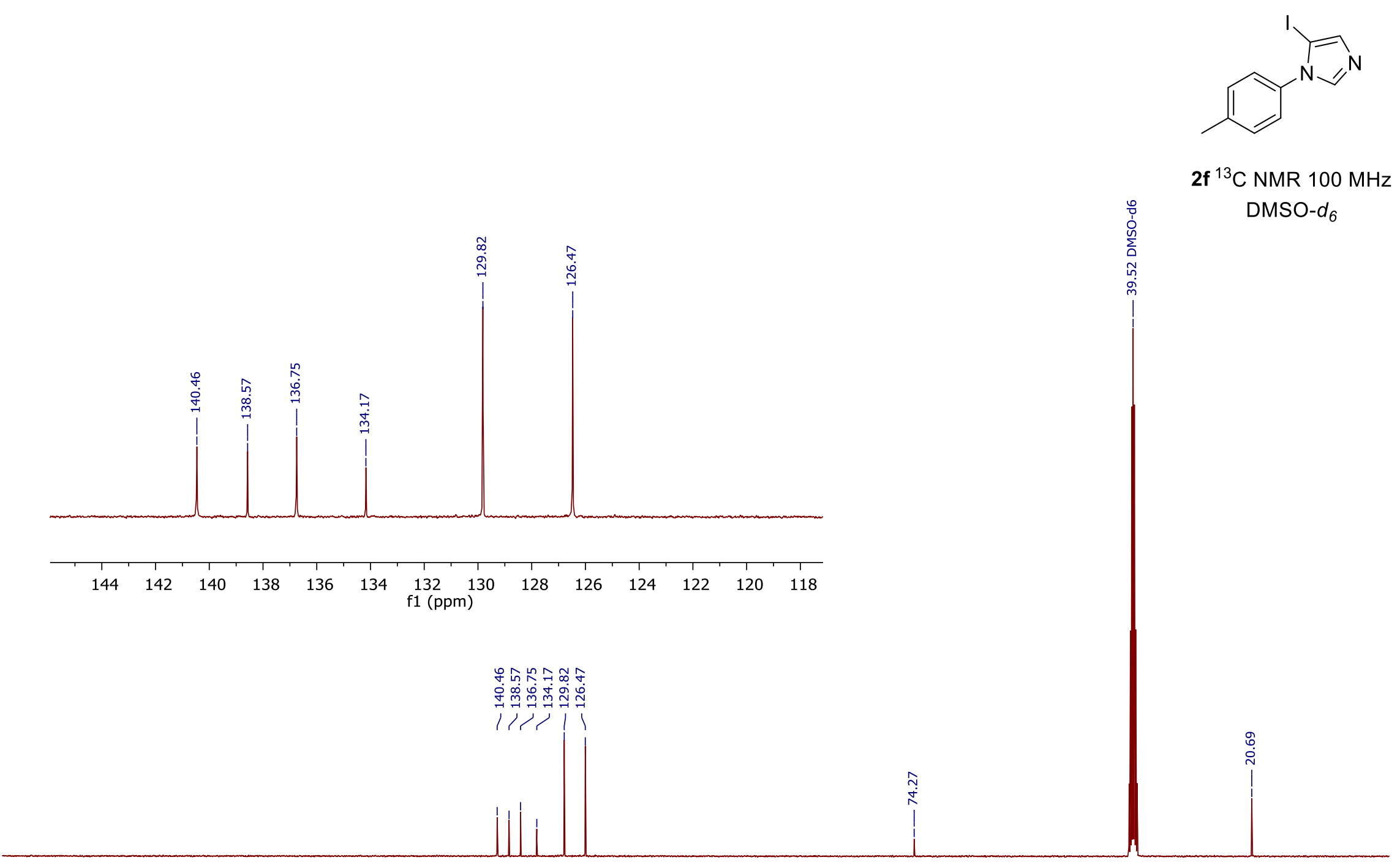

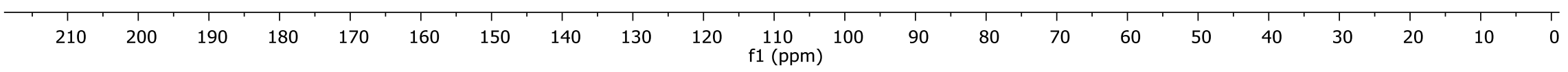




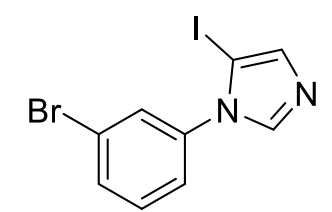

2g ${ }^{1} \mathrm{H}$ NMR $400 \mathrm{MHz}$ DMSO- $d_{6}$
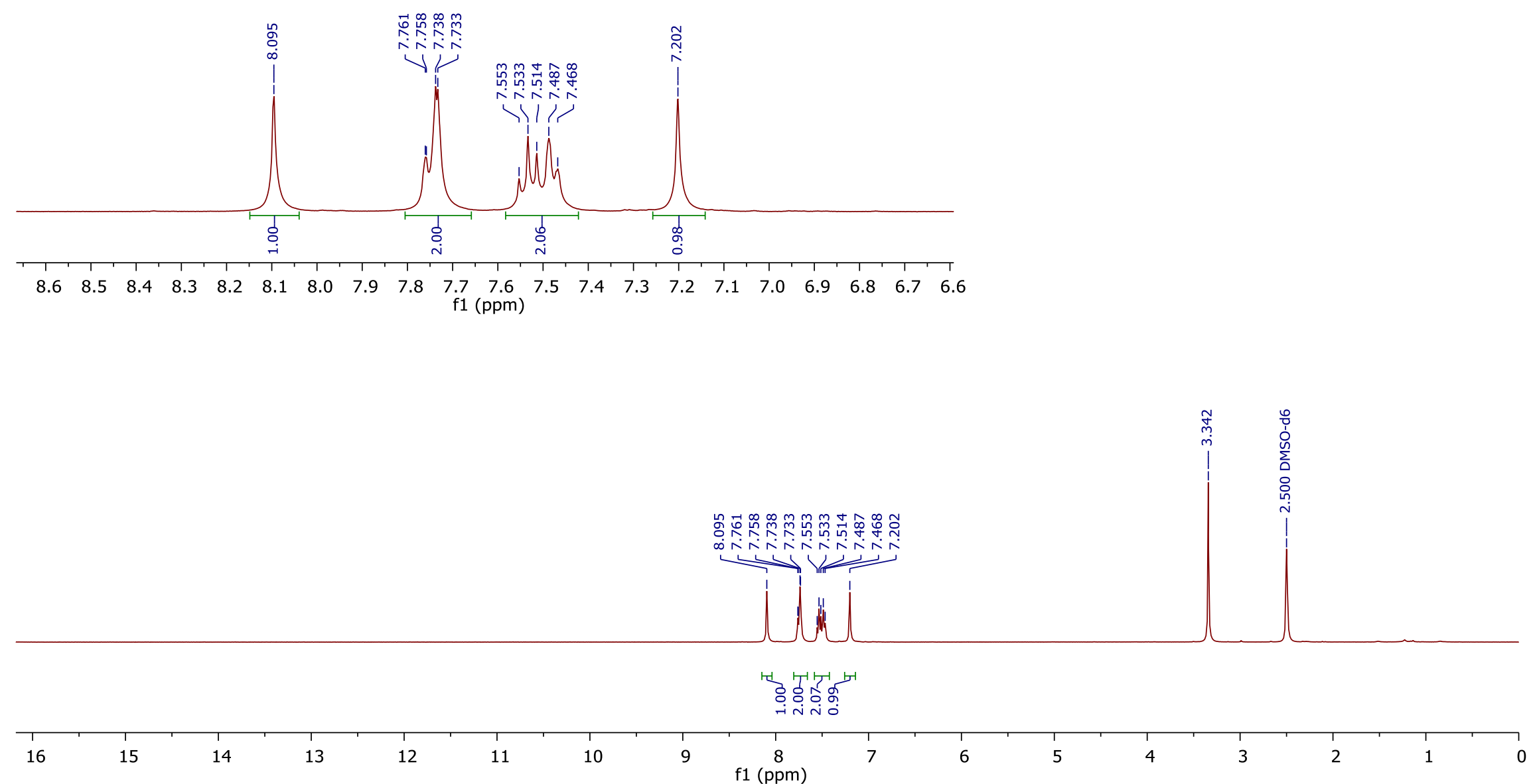


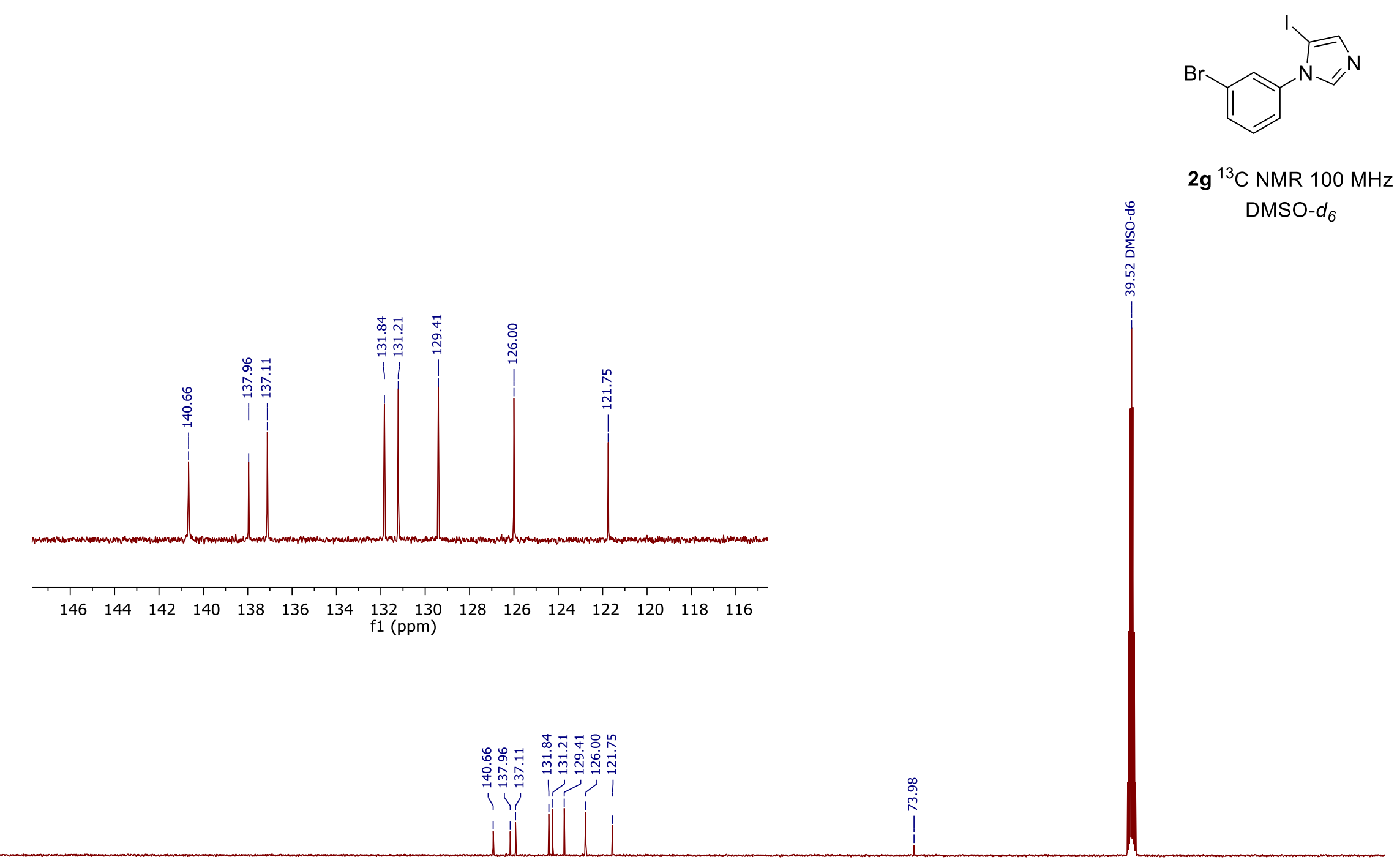

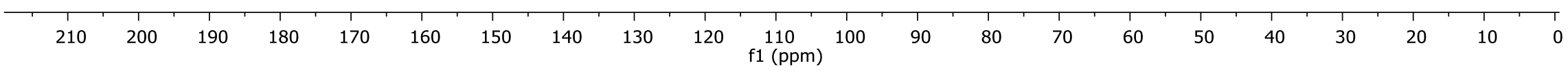




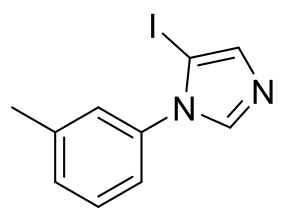

2h ${ }^{1} \mathrm{H}$ NMR $400 \mathrm{MHz}$ MeOD- $d_{4}$
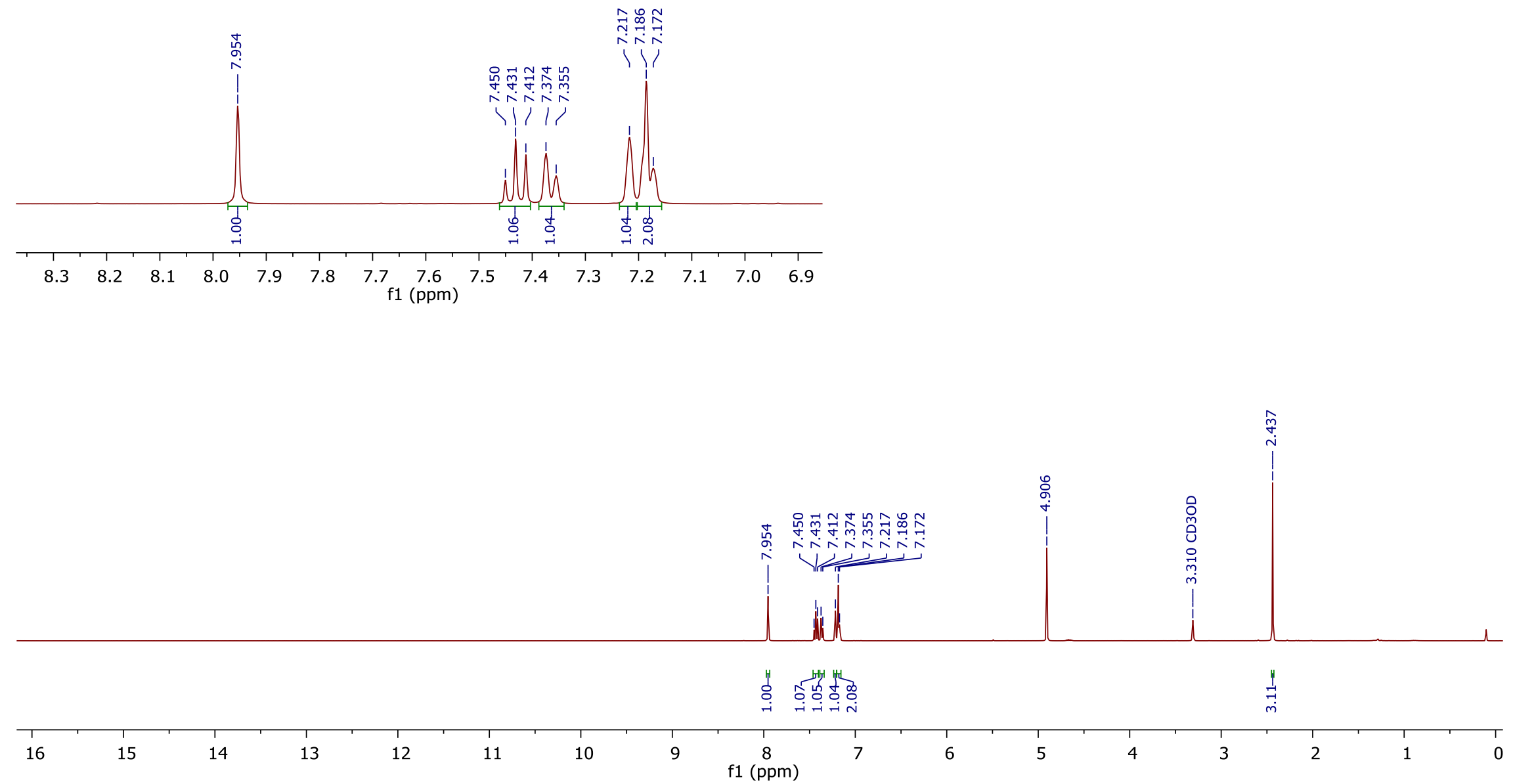


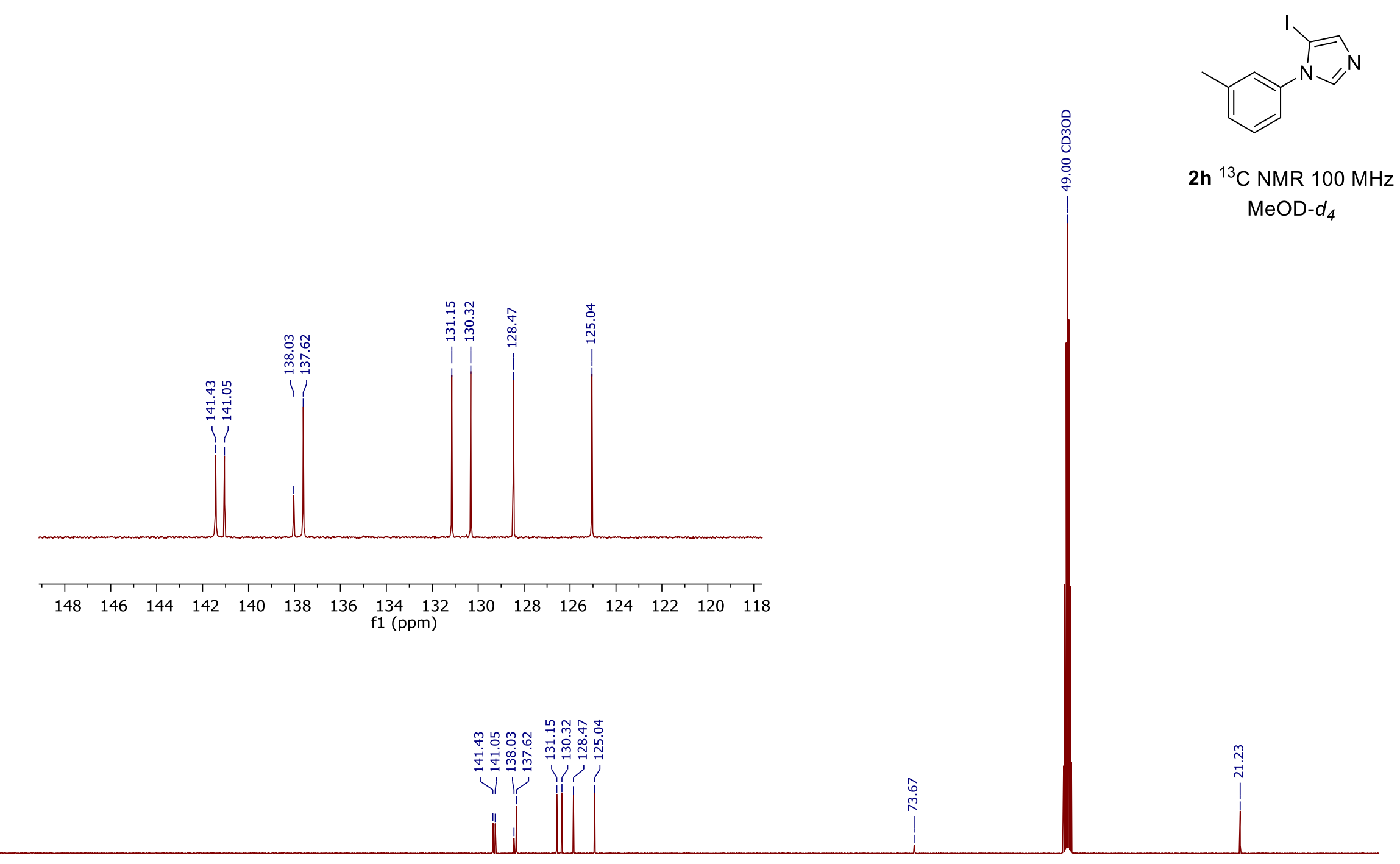

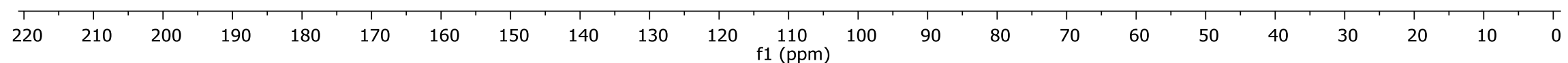




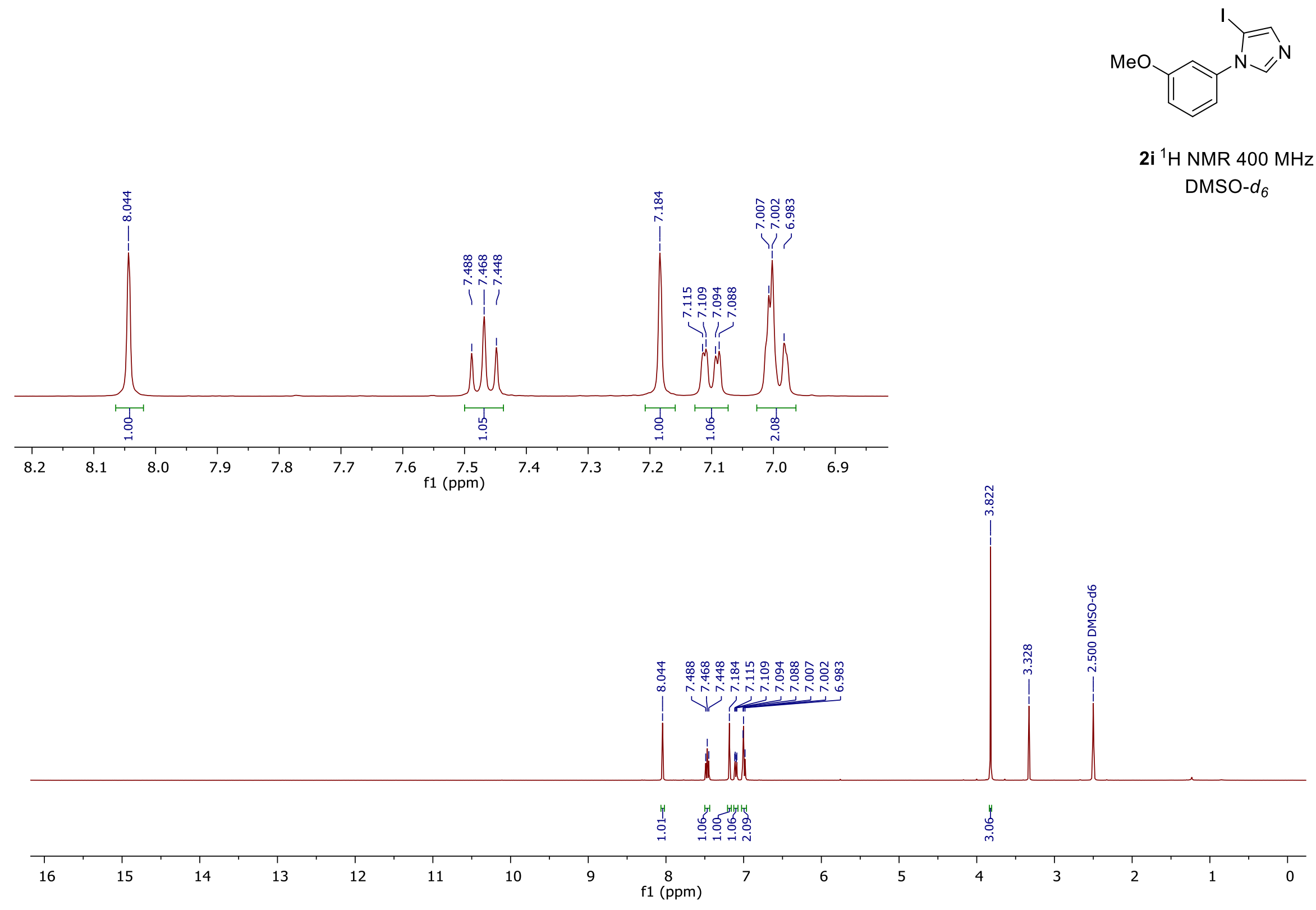




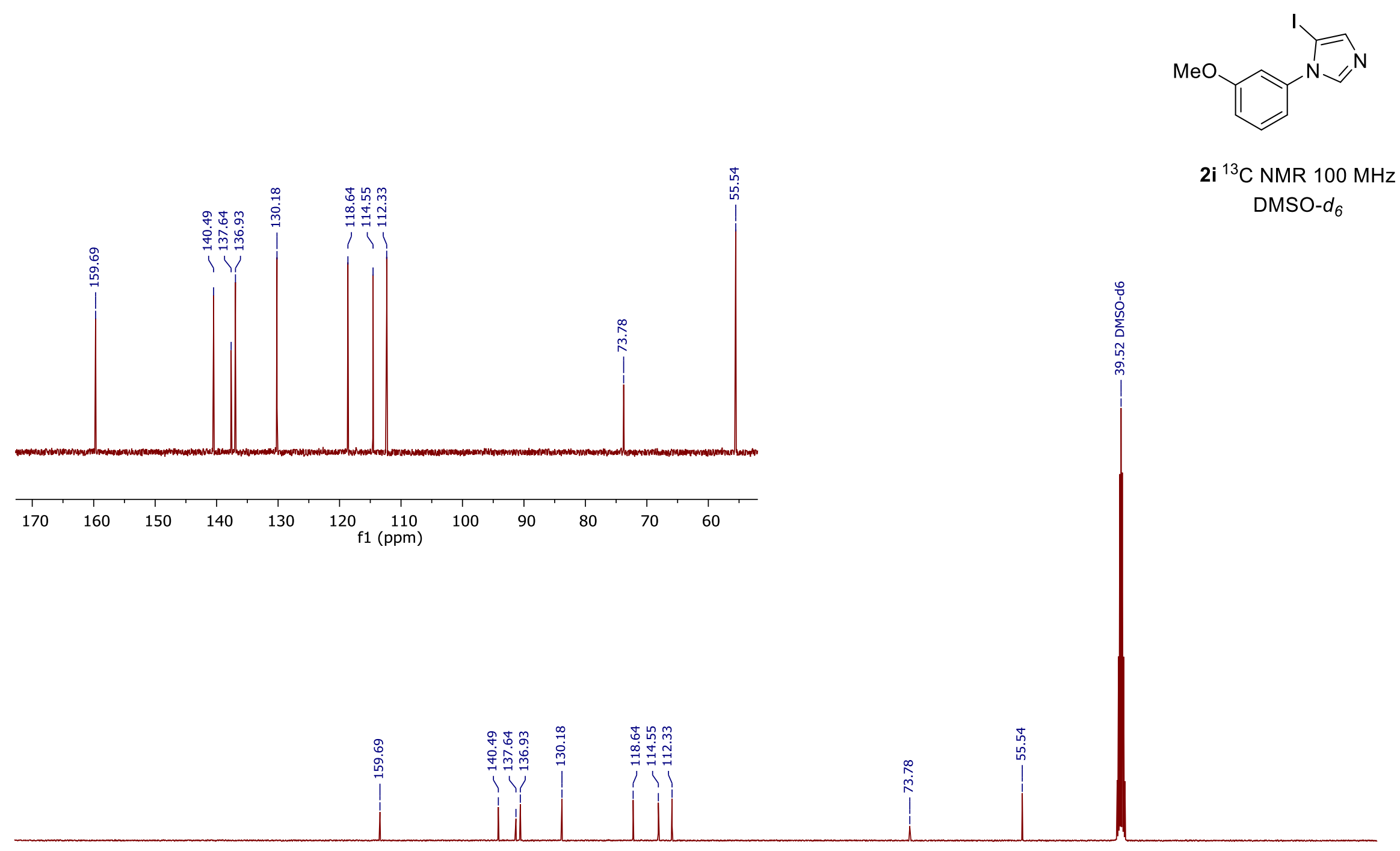

\begin{tabular}{lllllllllllllllllllllll}
\hline & 1 \\
210 & 200 & 190 & 180 & 170 & 160 & 150 & 140 & 130 & 120 & $\begin{array}{c}110 \\
\mathrm{f}(\mathrm{ppm})\end{array}$ & 100 & 90 & 80 & 70 & 60 & 50 & 40 & 30 & 20 & 10 & 0
\end{tabular}




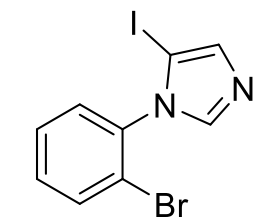

2j ${ }^{1} \mathrm{H}$ NMR $400 \mathrm{MHz}$ MeOD- $d_{4}$
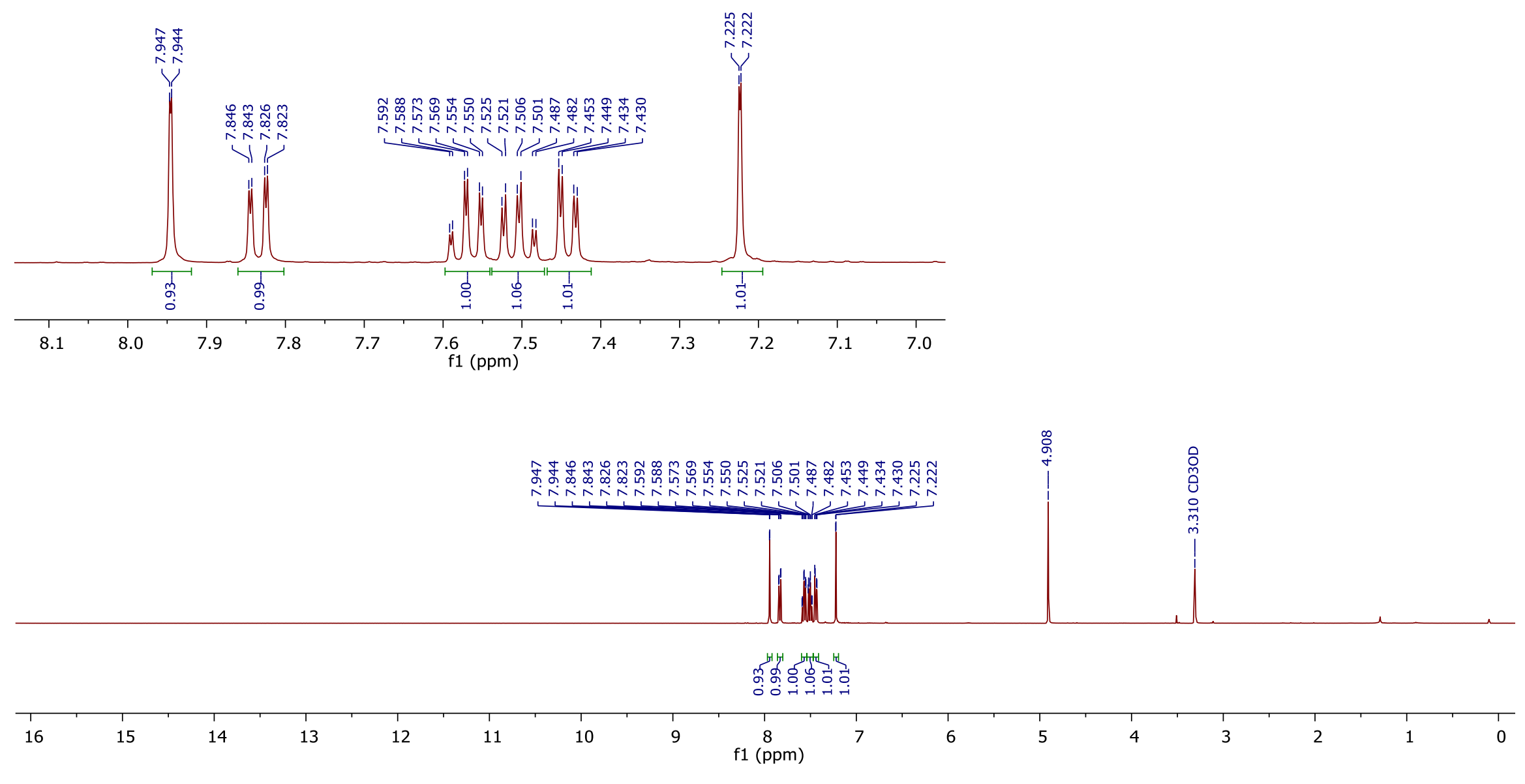


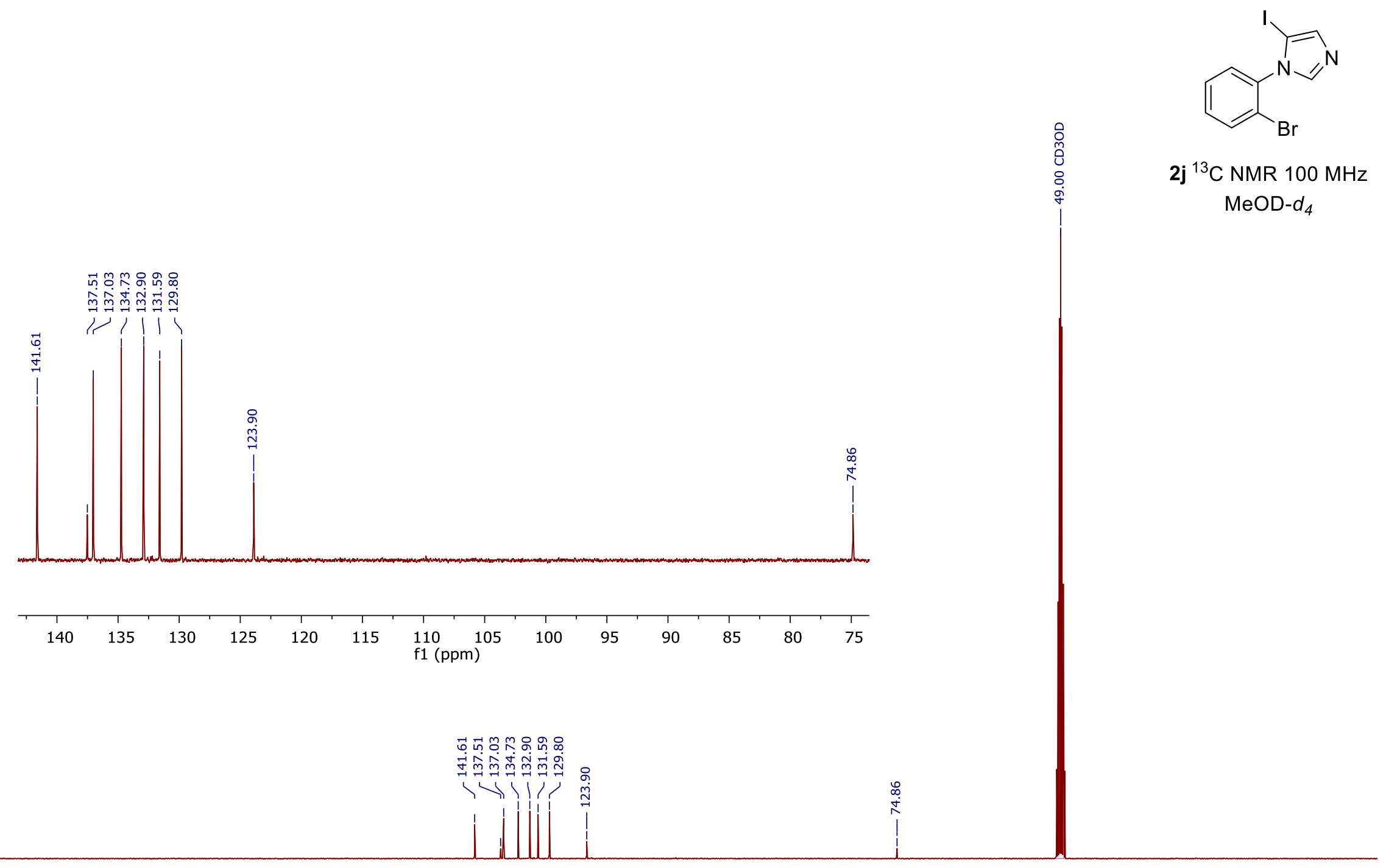

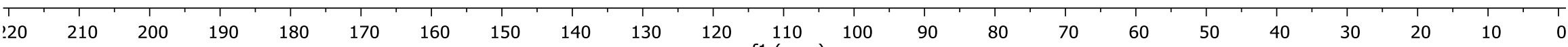




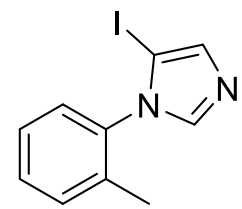

2k ${ }^{1} \mathrm{H}$ NMR $400 \mathrm{MHz}$ DMSO- $d_{6}$

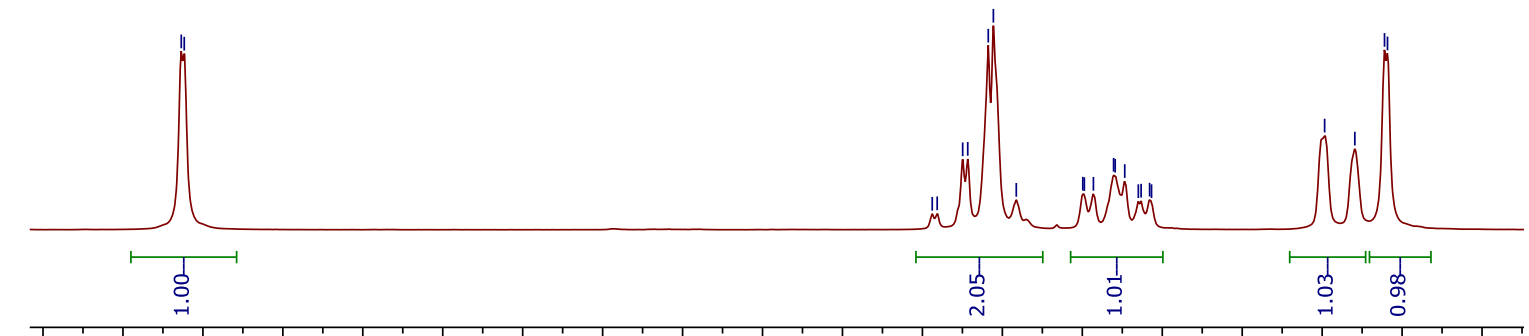

$\begin{array}{lllllllllllllllllll}8.05 & 8.00 & 7.95 & 7.90 & 7.85 & 7.80 & 7.75 & 7.70 & 7.65 & 7.60 & 7.55 & 7.50 & 7.45 & 7.40 & 7.35 & 7.30 & 7.25 & 7.20 & 7.15\end{array}$ f1 (ppm)

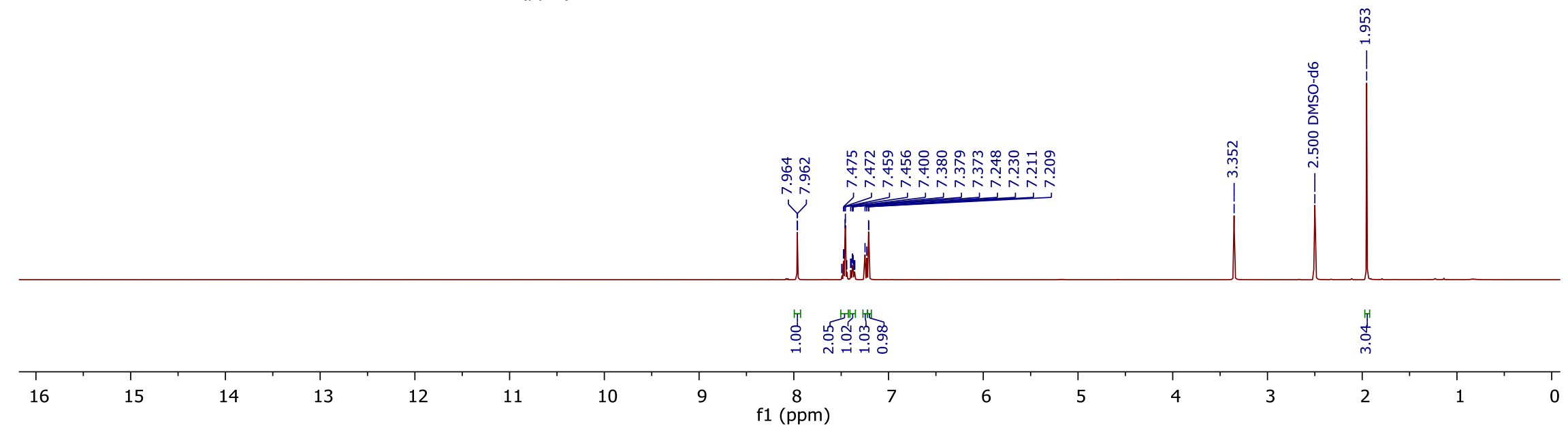




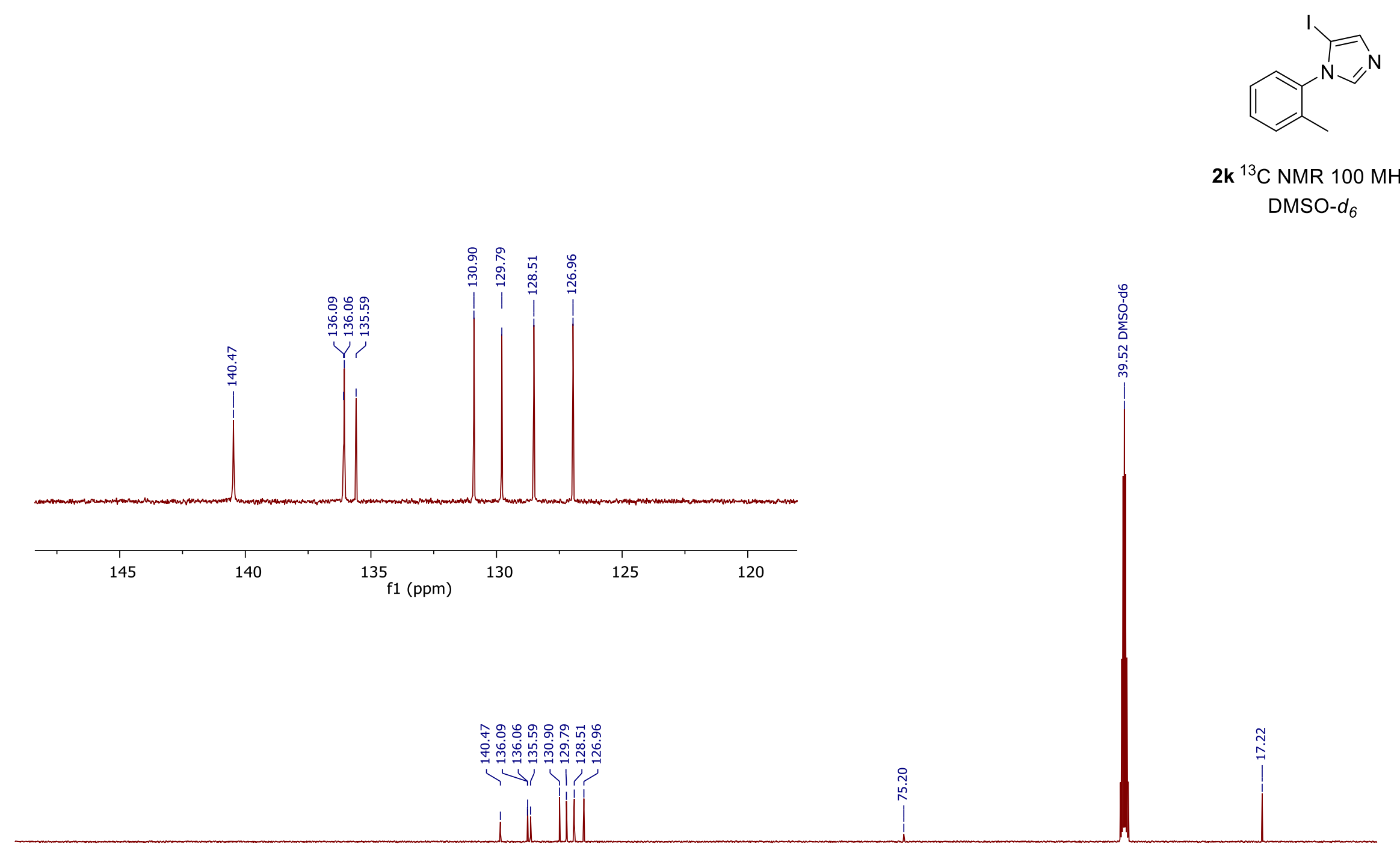

\begin{tabular}{llllllllllllllllllllll}
\hline 210 & 200 & 190 & 180 & 170 & 160 & 150 & 140 & 130 & 120 & $\begin{array}{c}110 \\
\mathrm{f} 1(\mathrm{ppm})\end{array}$ & 100 & 90 & 80 & 70 & 60 & 50 & 40 & 30 & 20 & 10 & 0
\end{tabular}




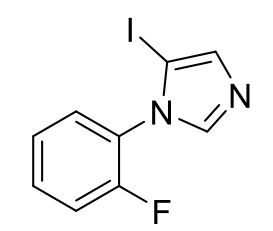

2I ${ }^{1} \mathrm{H}$ NMR $400 \mathrm{MHz}$ MeOD- $d_{4}$
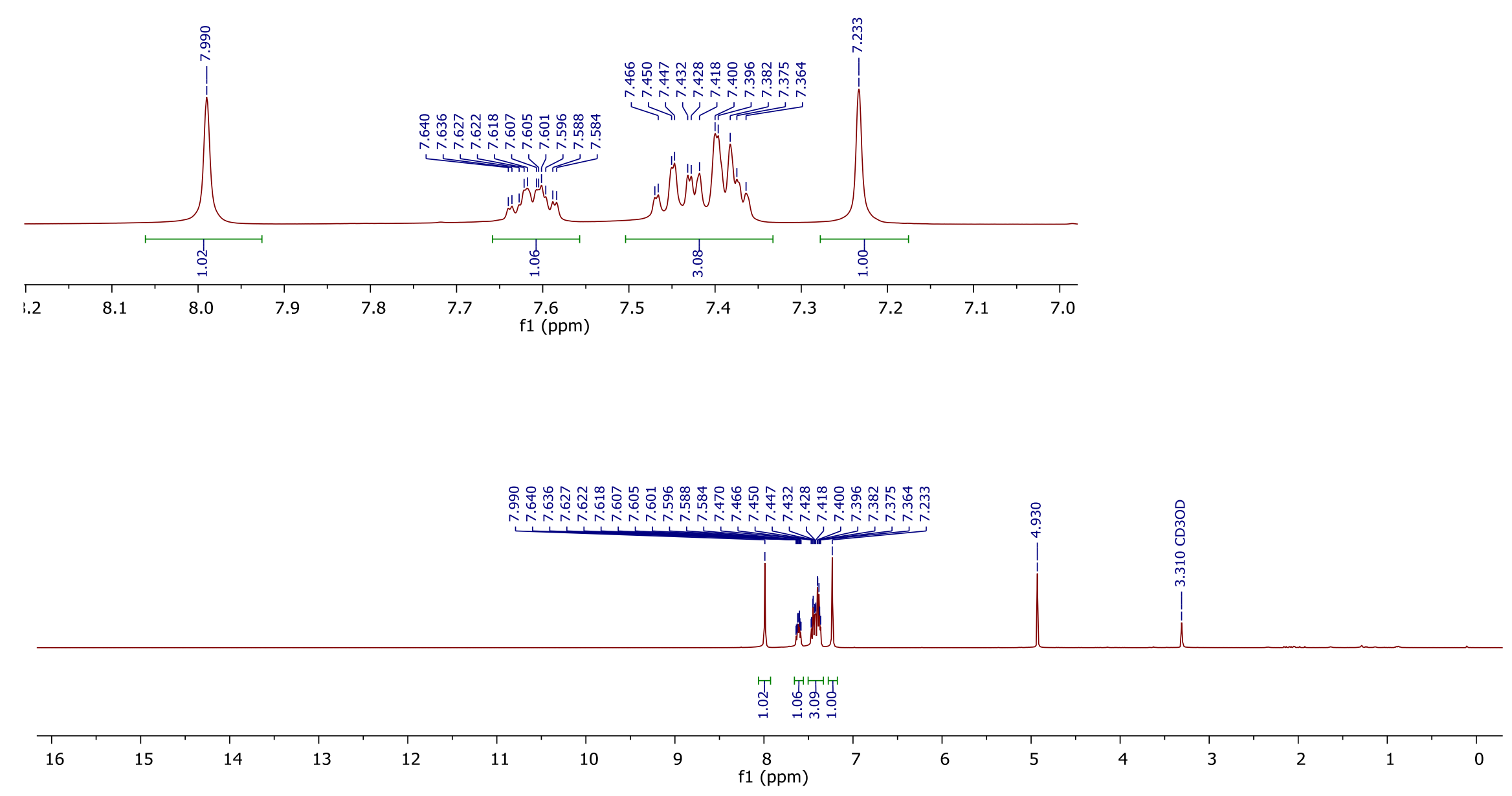


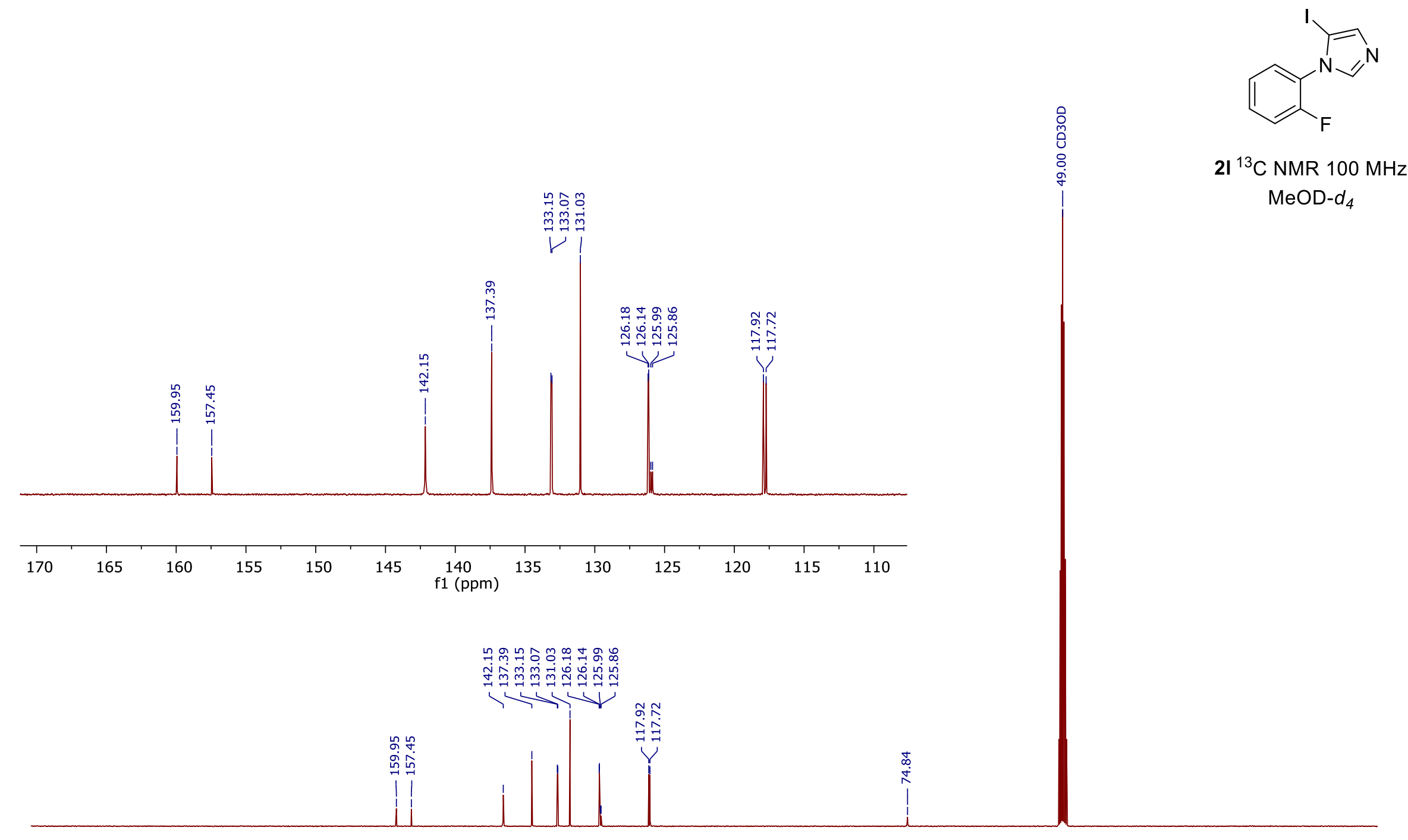

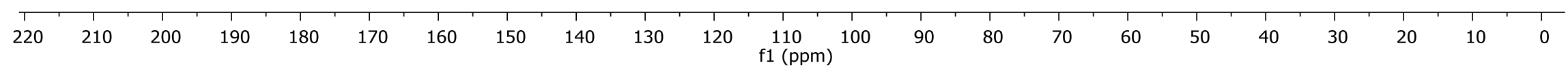




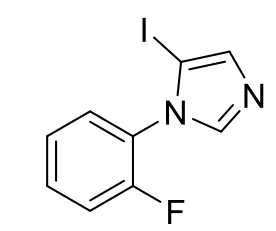

2I ${ }^{19} \mathrm{~F}$ NMR $376 \mathrm{MHz}$ $\mathrm{MeOD}-d_{4}$

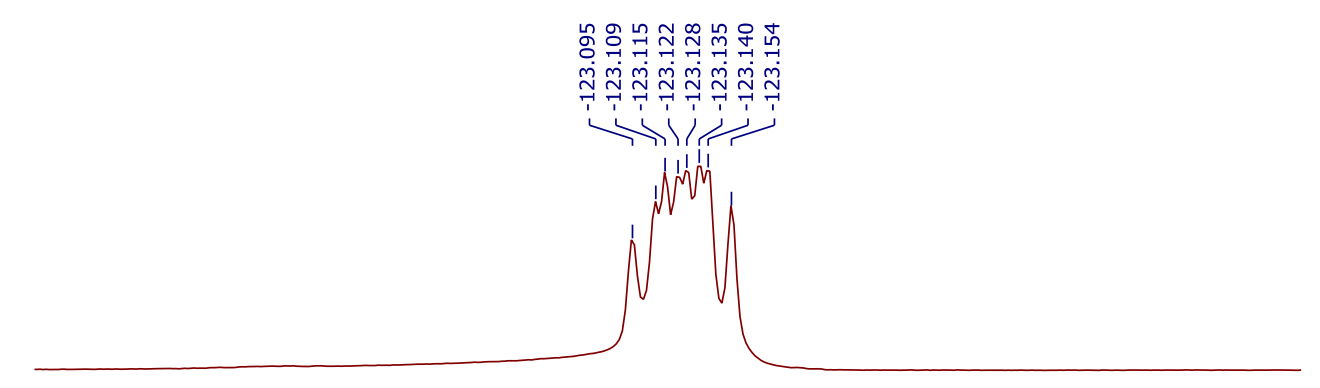

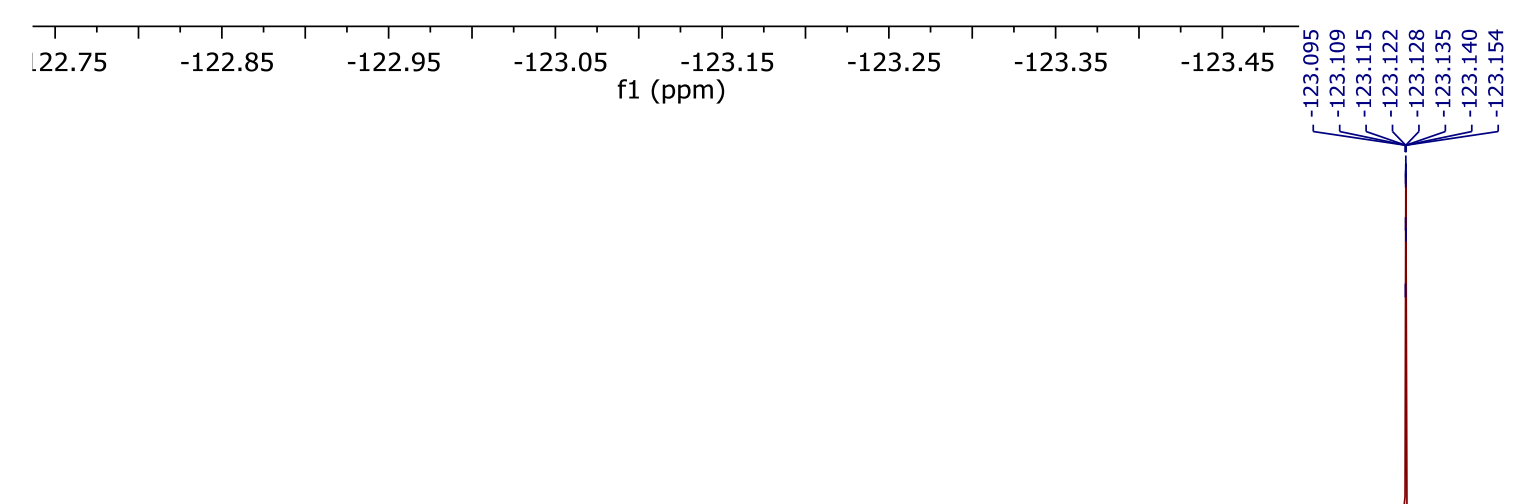

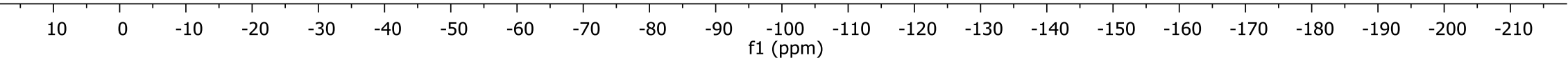




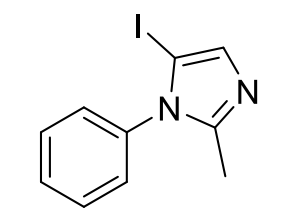

$2 \mathrm{~m}^{1} \mathrm{H}$ NMR $400 \mathrm{MHz}$ DMSO- $d_{6}$
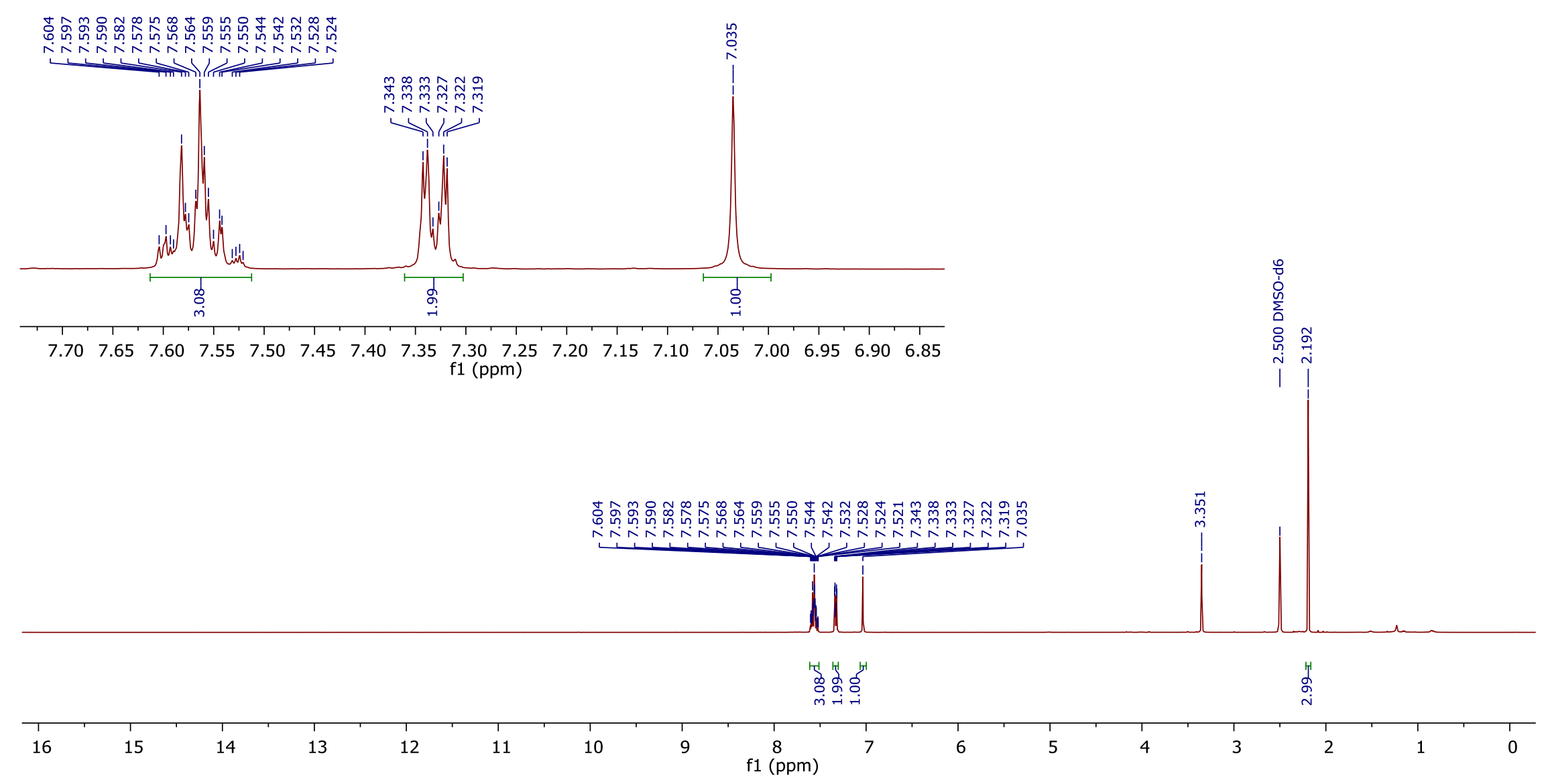

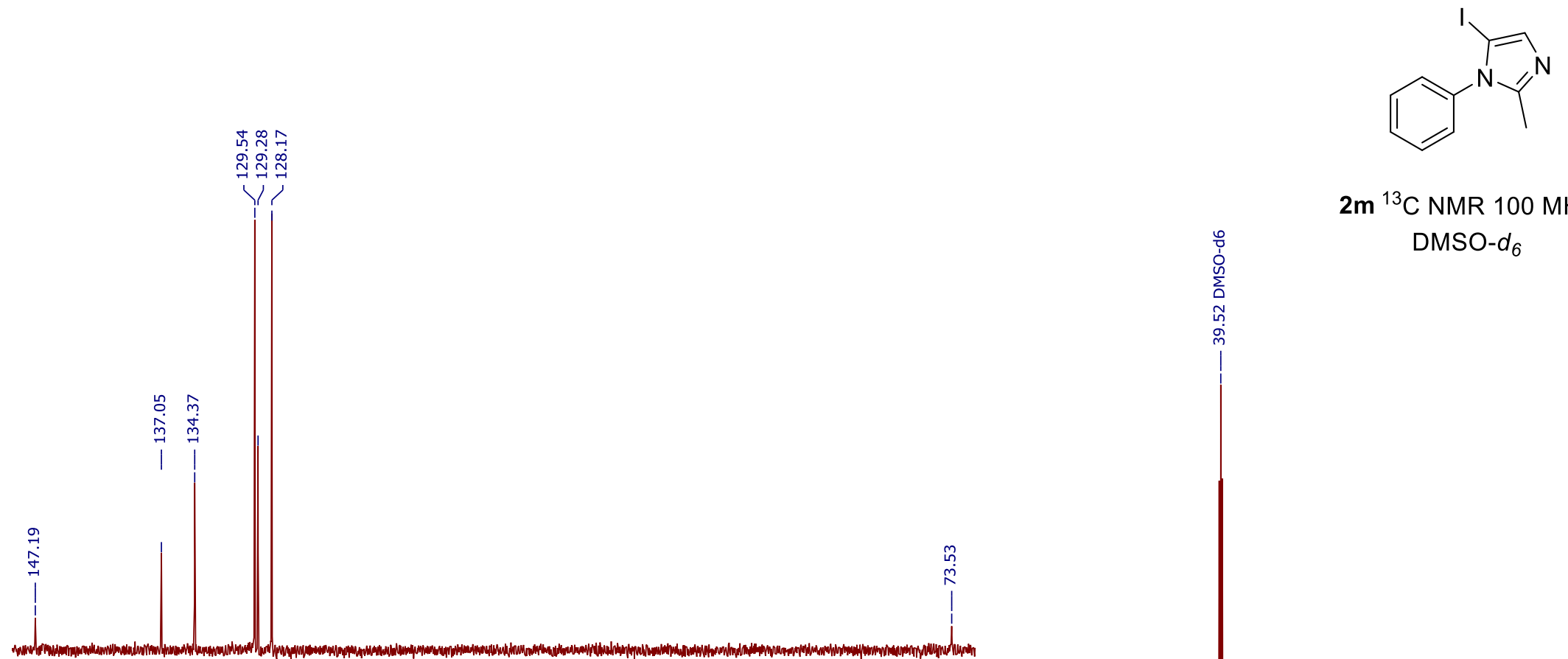

$2 \mathrm{~m}^{13} \mathrm{C}$ NMR $100 \mathrm{MHz}$

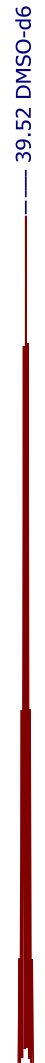
DMSO-d $d_{6}$

$\begin{array}{lllllllllllllll}145 & 140 & 135 & 130 & 125 & 120 & 115 \underset{f 1}{110} & 105 & 100 & 95 & 90 & 85 & 80 & 75\end{array}$

นุำ

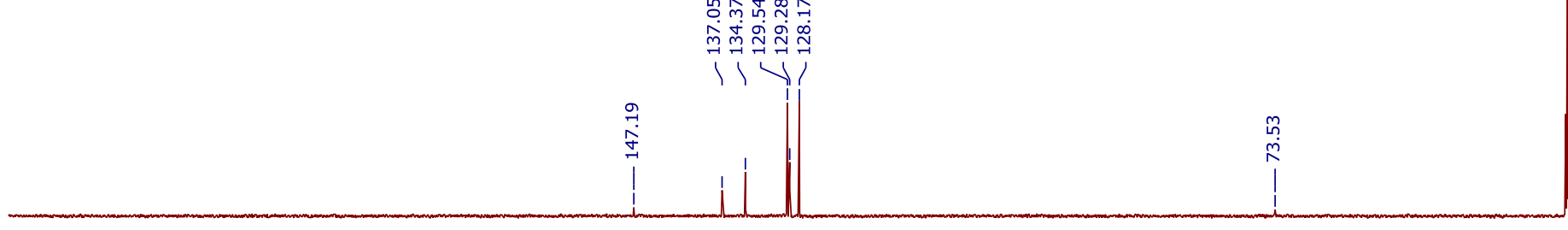

$\stackrel{\substack{+:}}{+}$

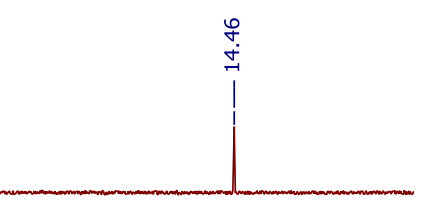

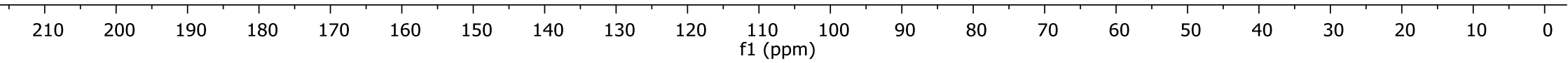




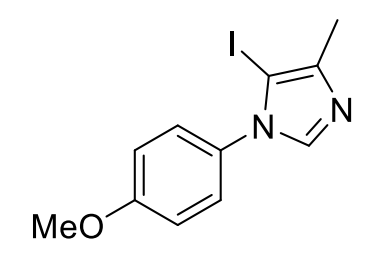

2n ${ }^{1} \mathrm{H}$ NMR $400 \mathrm{MHz}$ $\mathrm{DMSO}_{6} d_{6}$
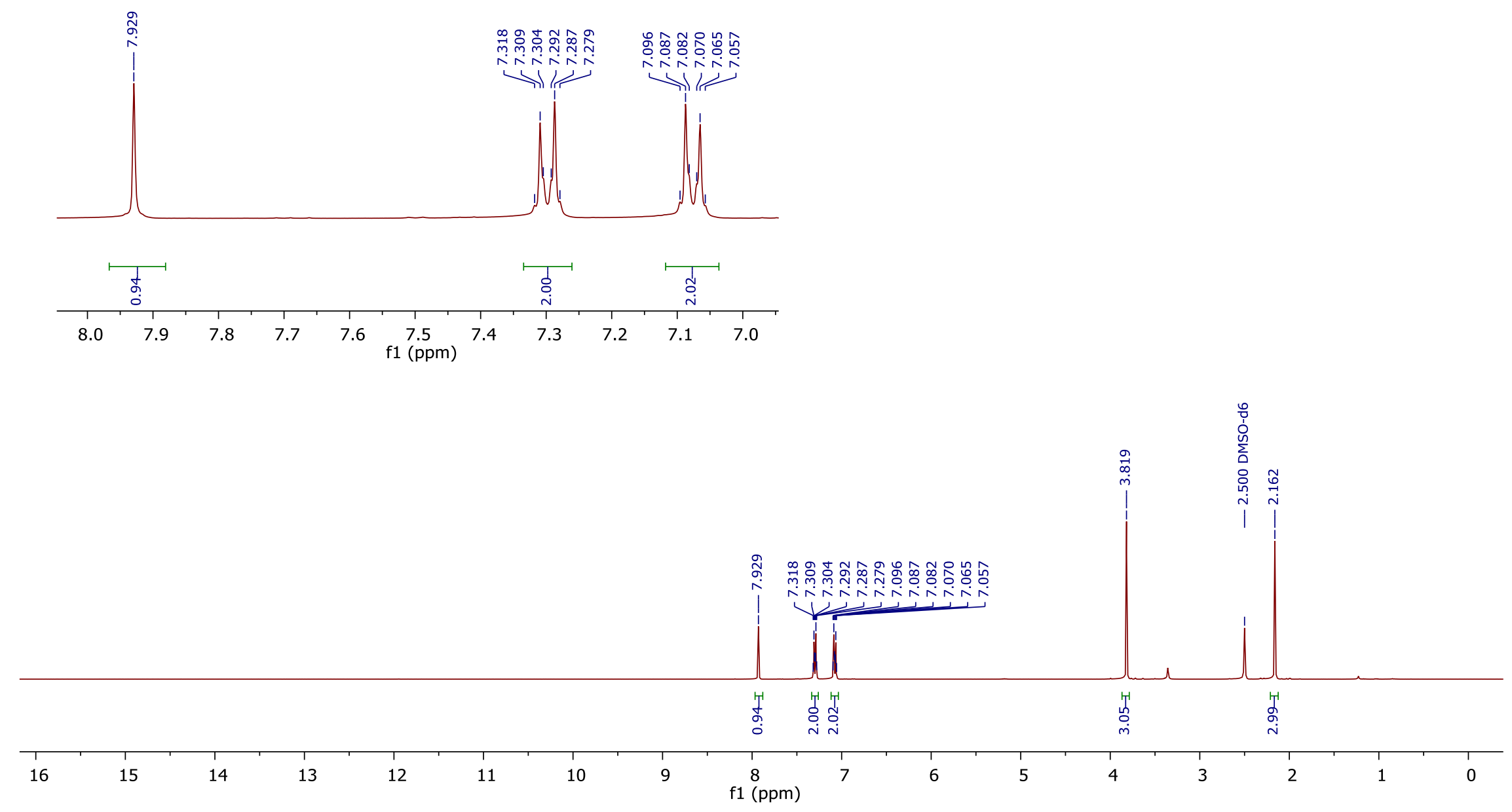


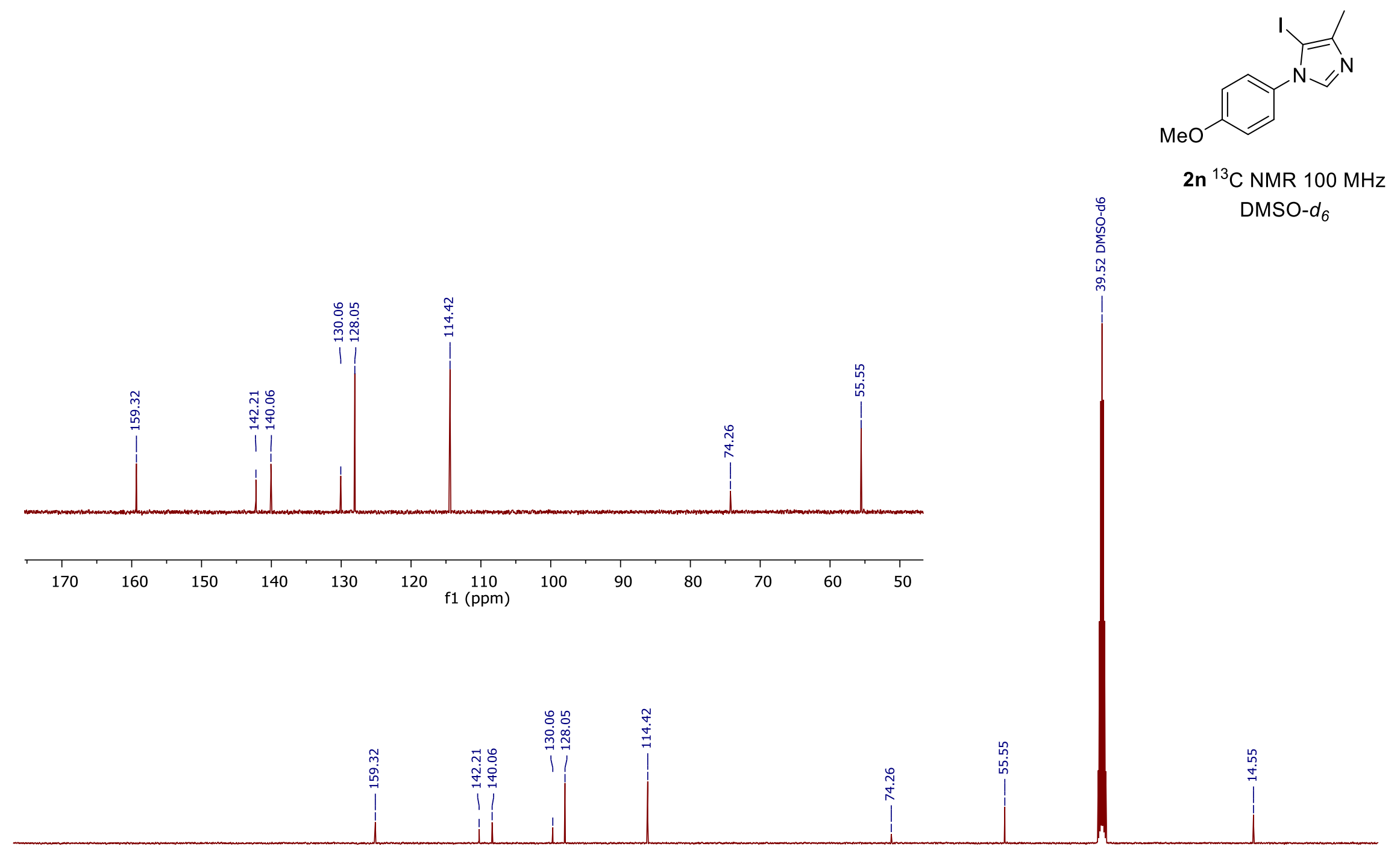

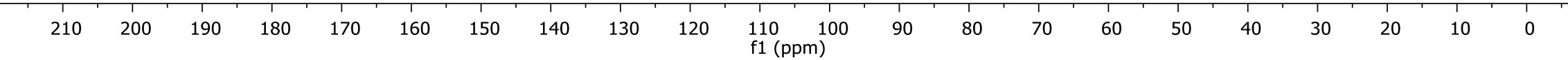




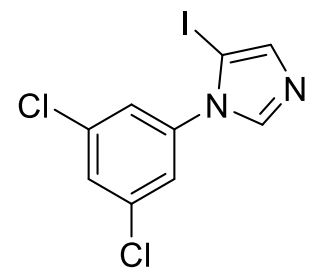

20 ${ }^{1} \mathrm{H}$ NMR $400 \mathrm{MHz}$ MeOD- $d_{4}$
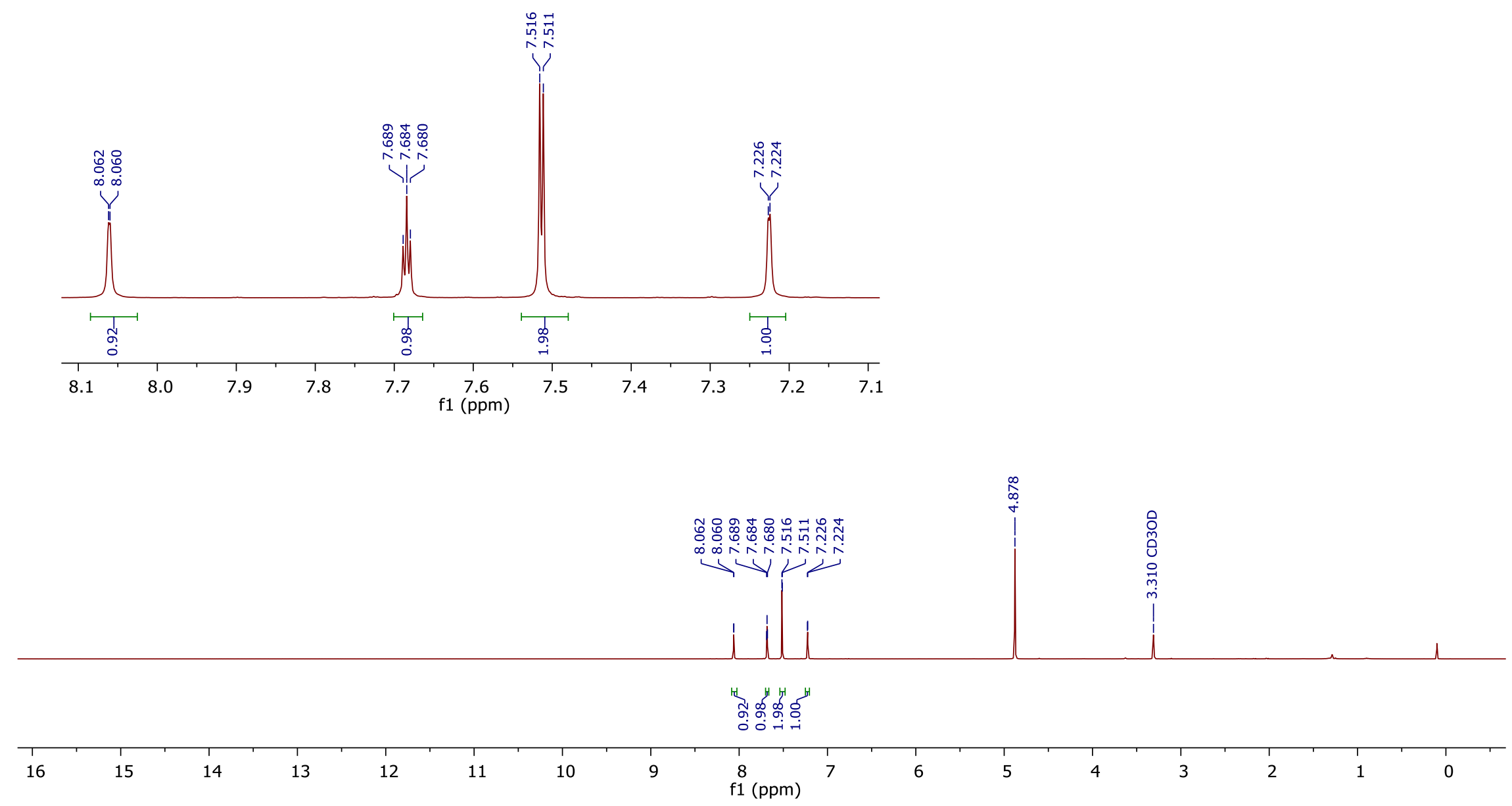


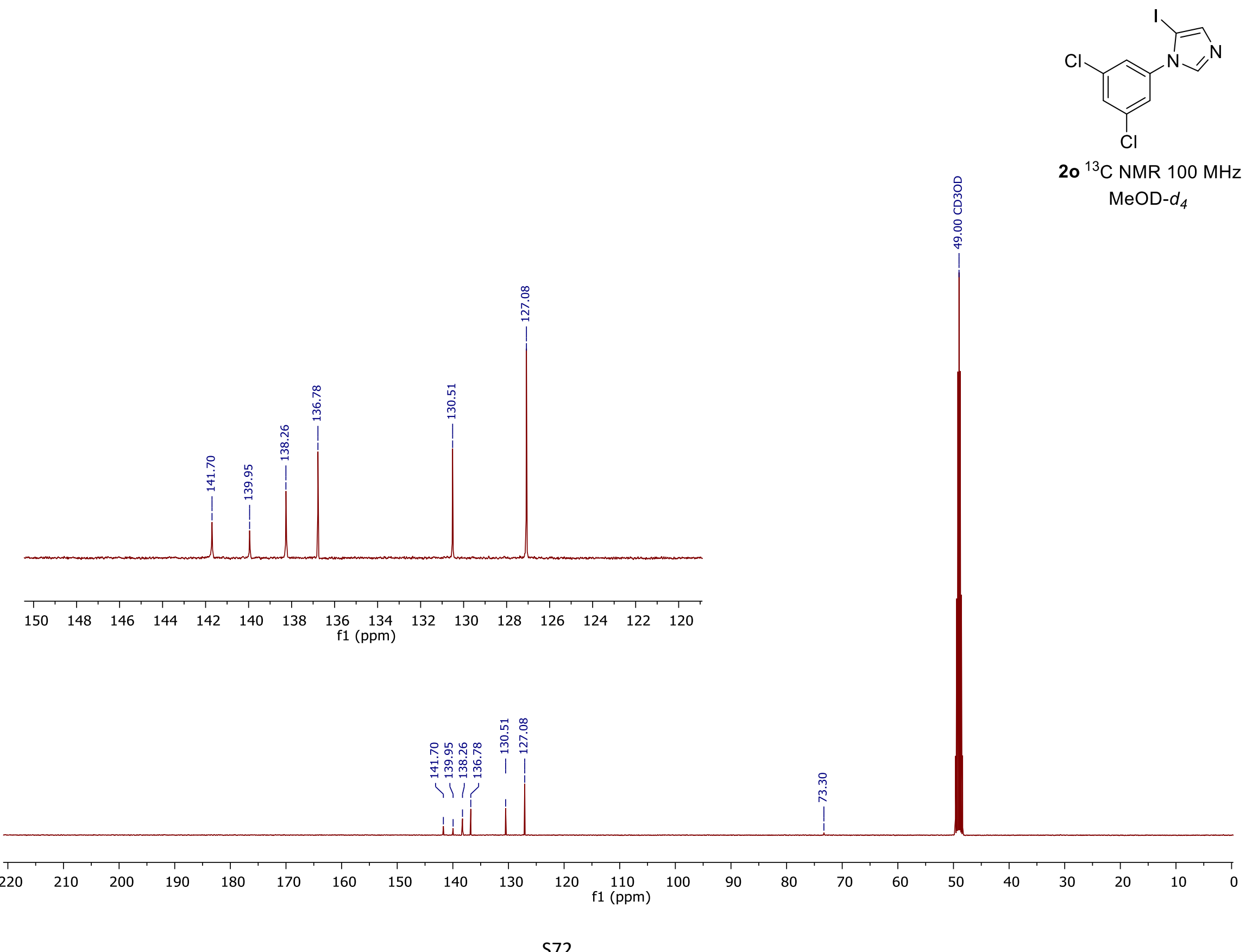




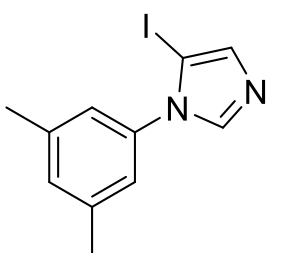

2p ${ }^{1} \mathrm{H}$ NMR $400 \mathrm{MHz}$ DMSO- $d_{6}$
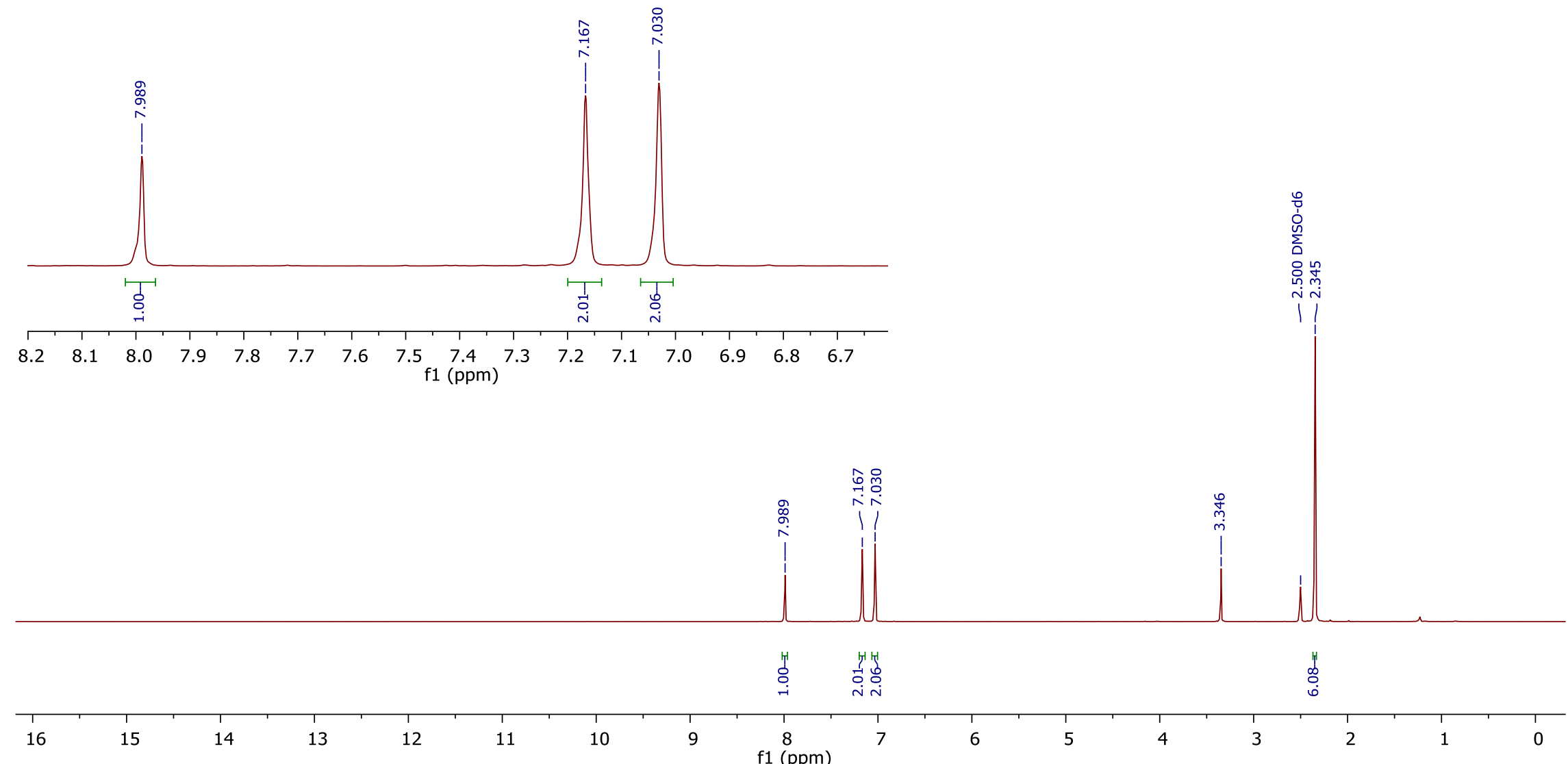


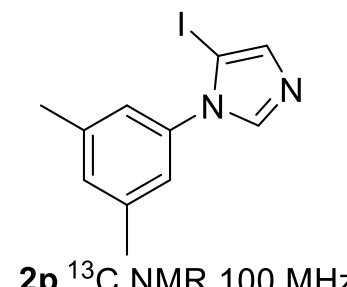
DMSO- $d_{6}$
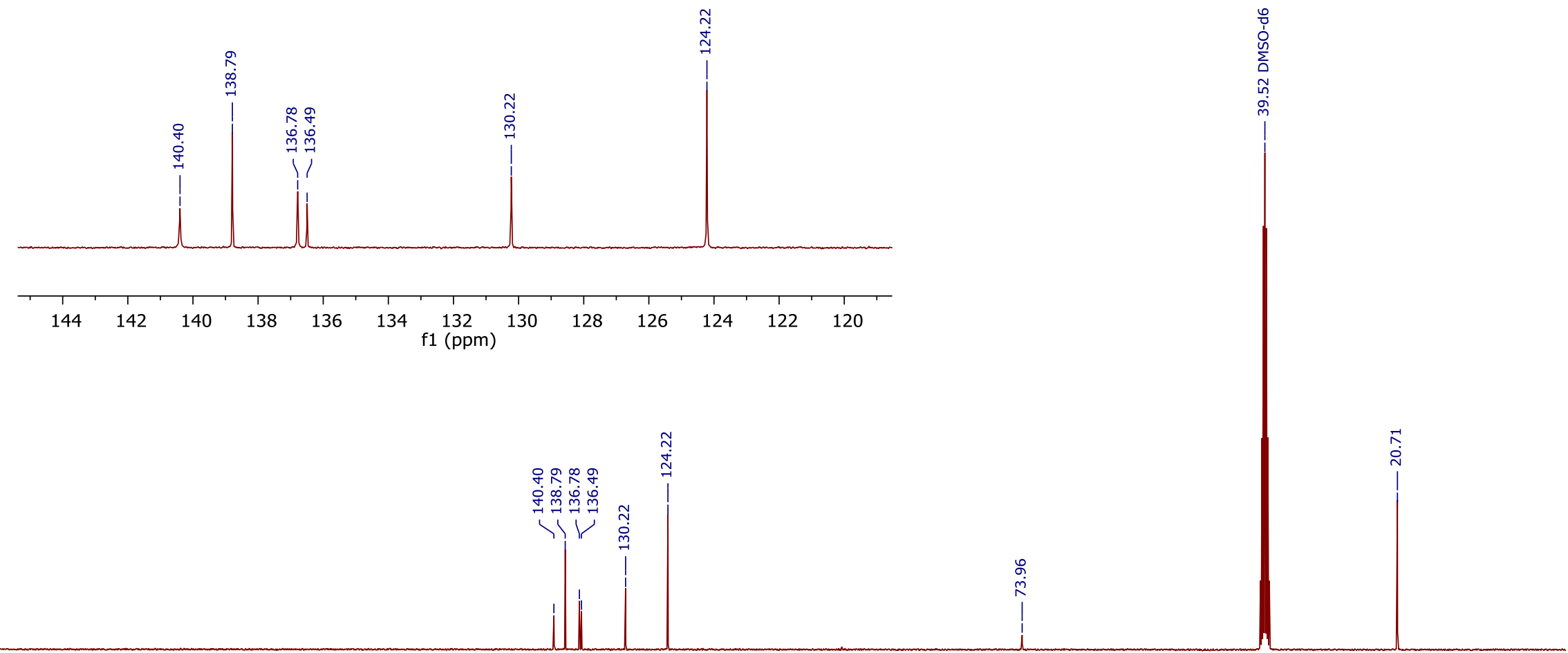

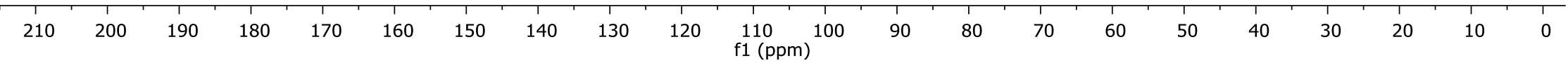




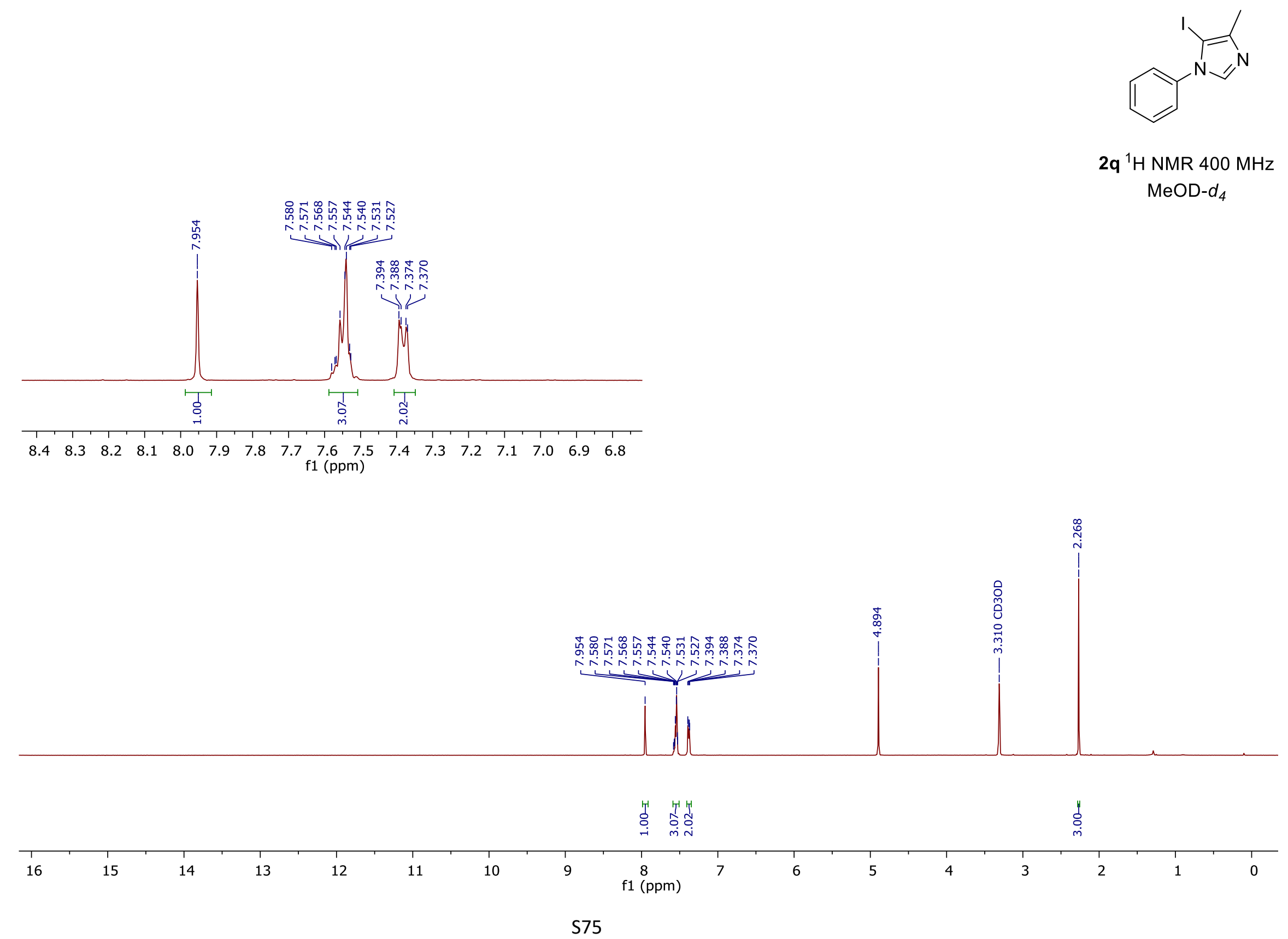



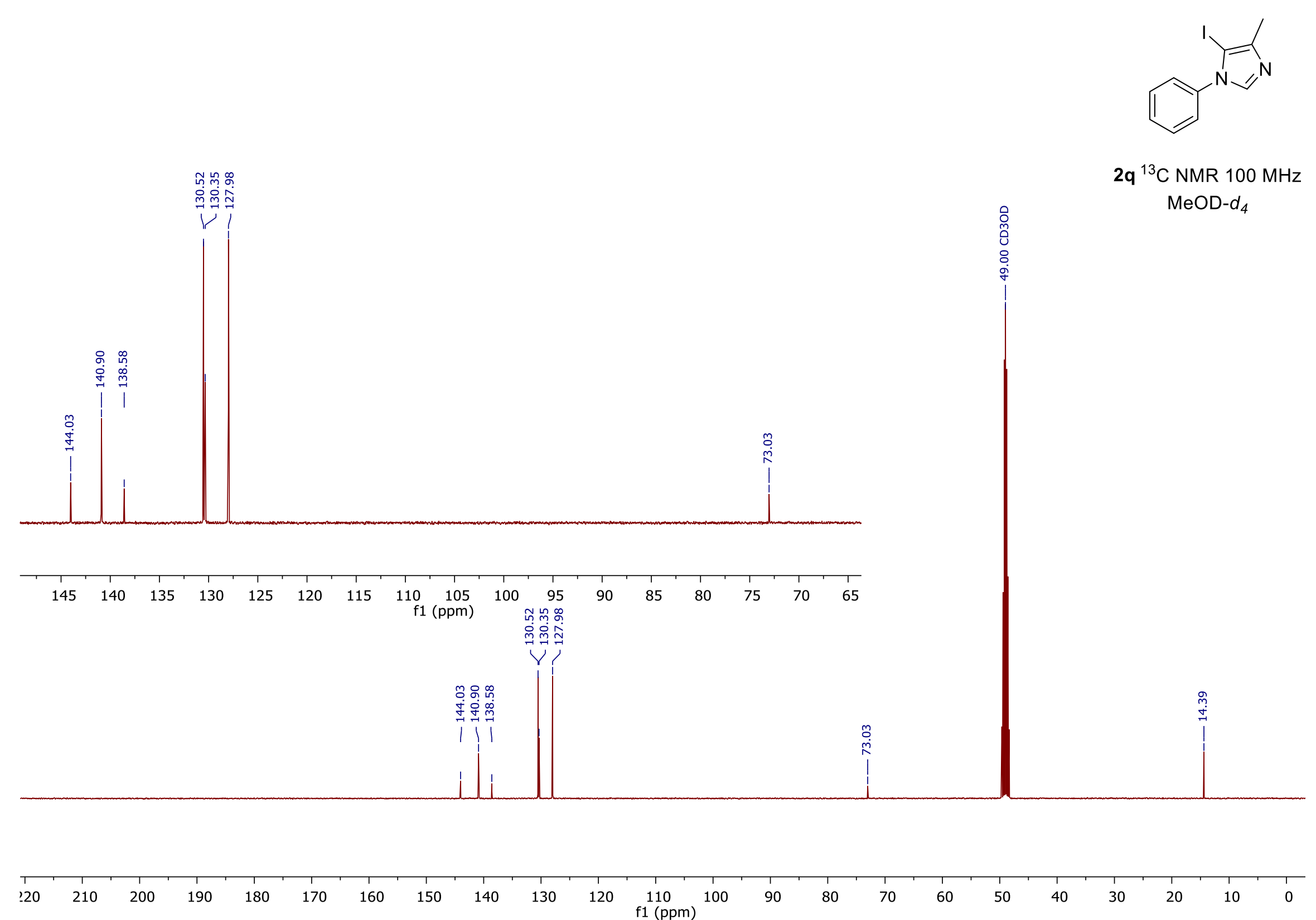

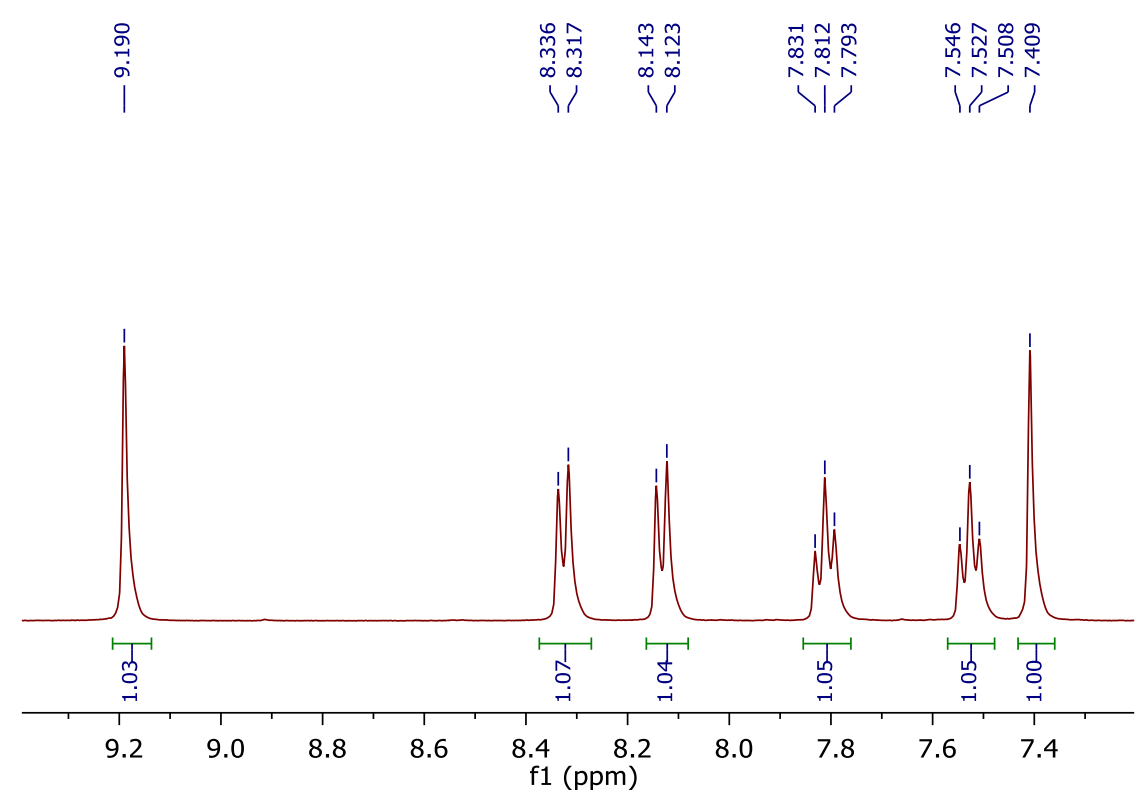

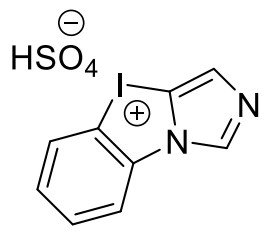

3a ${ }^{1} \mathrm{H}$ NMR $400 \mathrm{MHz}$ DMSO- $d_{6}$

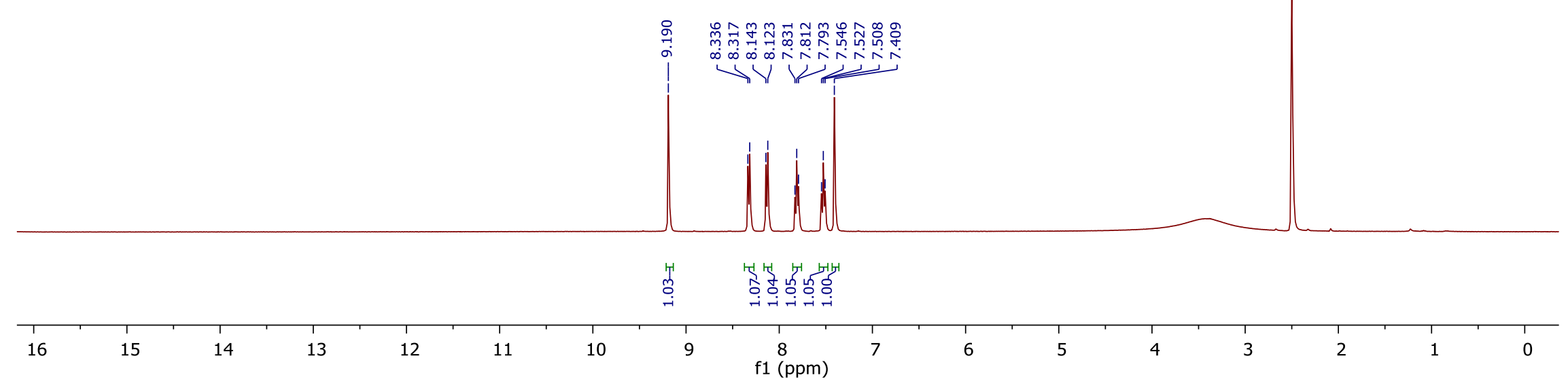




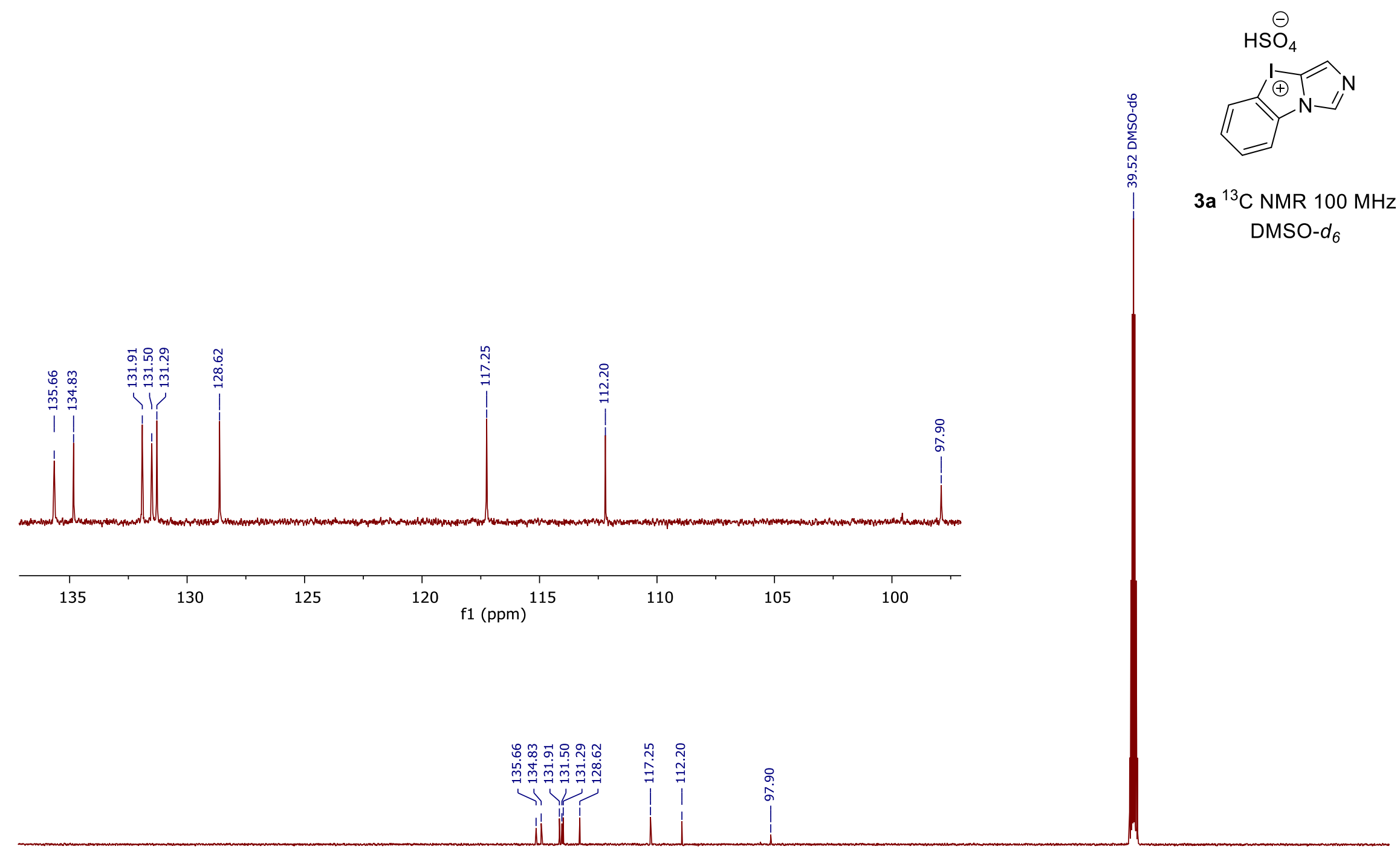

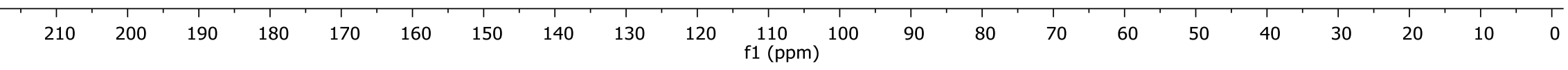


$\ominus$

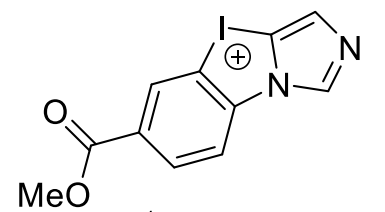

3b ${ }^{1} \mathrm{H}$ NMR $400 \mathrm{MHz}$ DMSO-d $d_{6}$

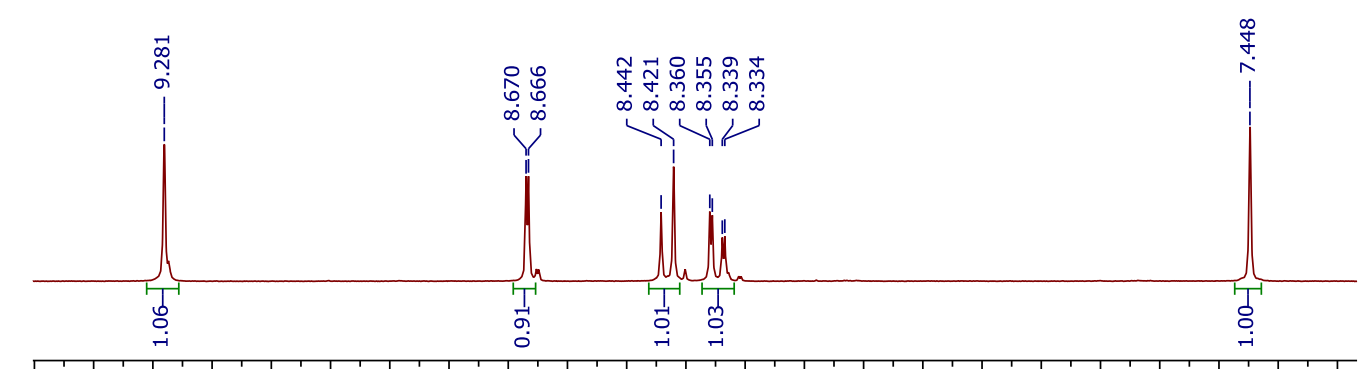
$\begin{array}{llllllllllllllllllllllll}1.5 & 9.4 & 9.3 & 9.2 & 9.1 & 9.0 & 8.9 & 8.8 & 8.7 & 8.6 & 8.5 & 8.4 & 8.3 & 8.2 & 8.1 & 8.0 & 7.9 & 7.8 & 7.7 & 7.6 & 7.5 & 7.4 & 7.3\end{array}$

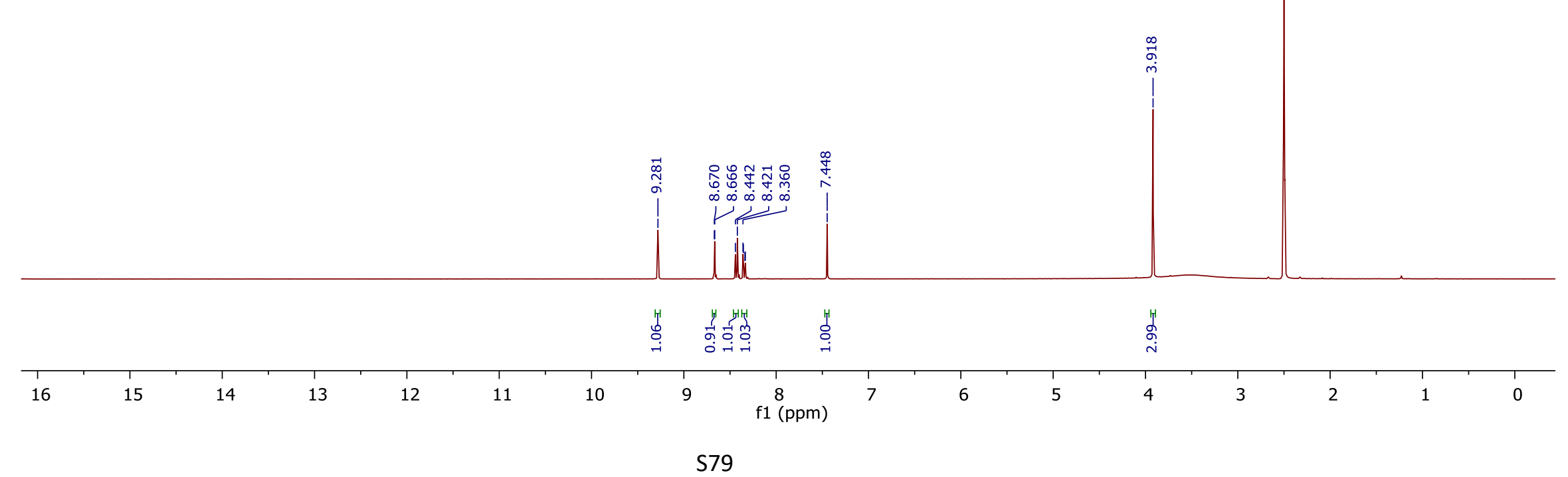




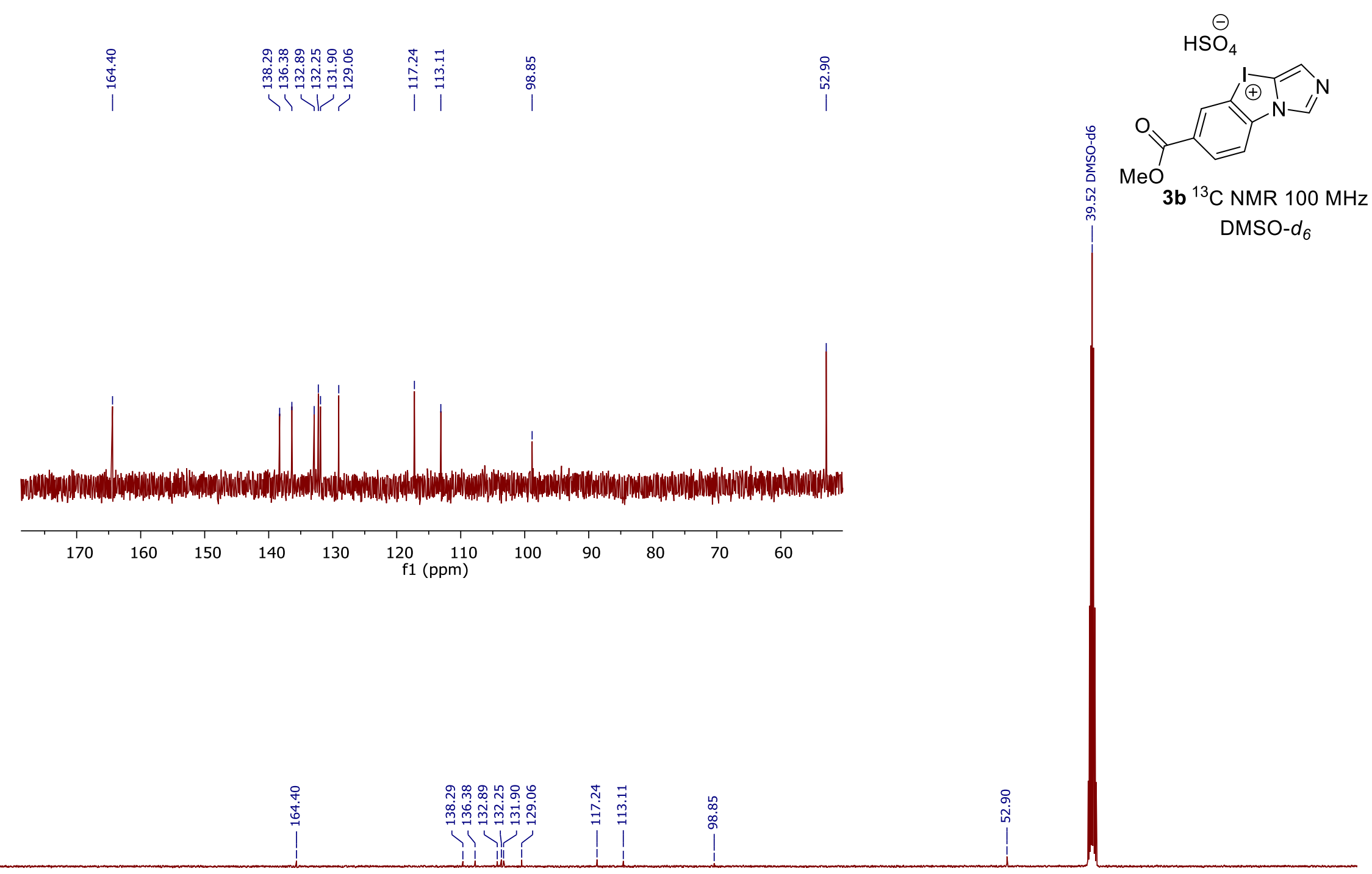




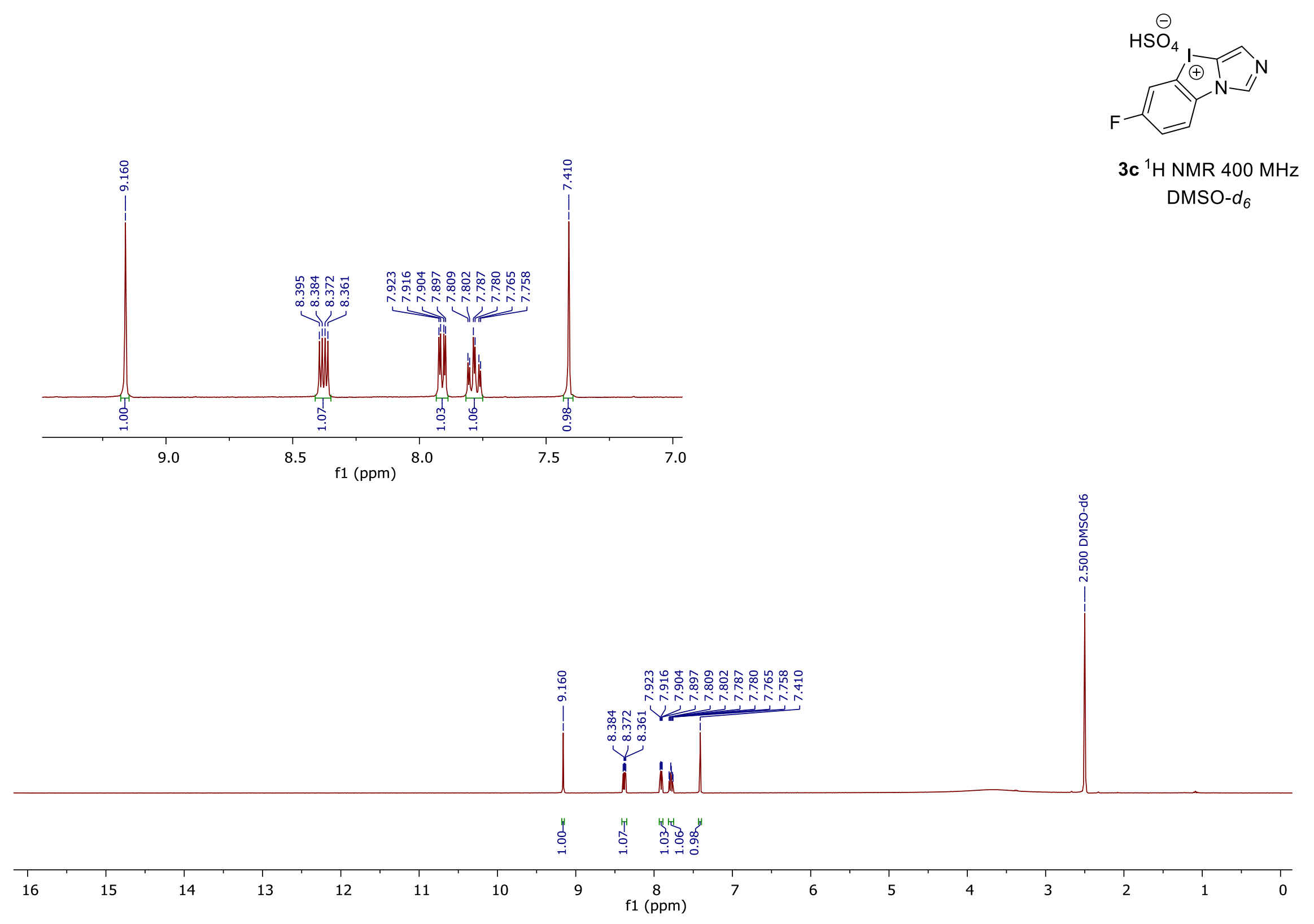




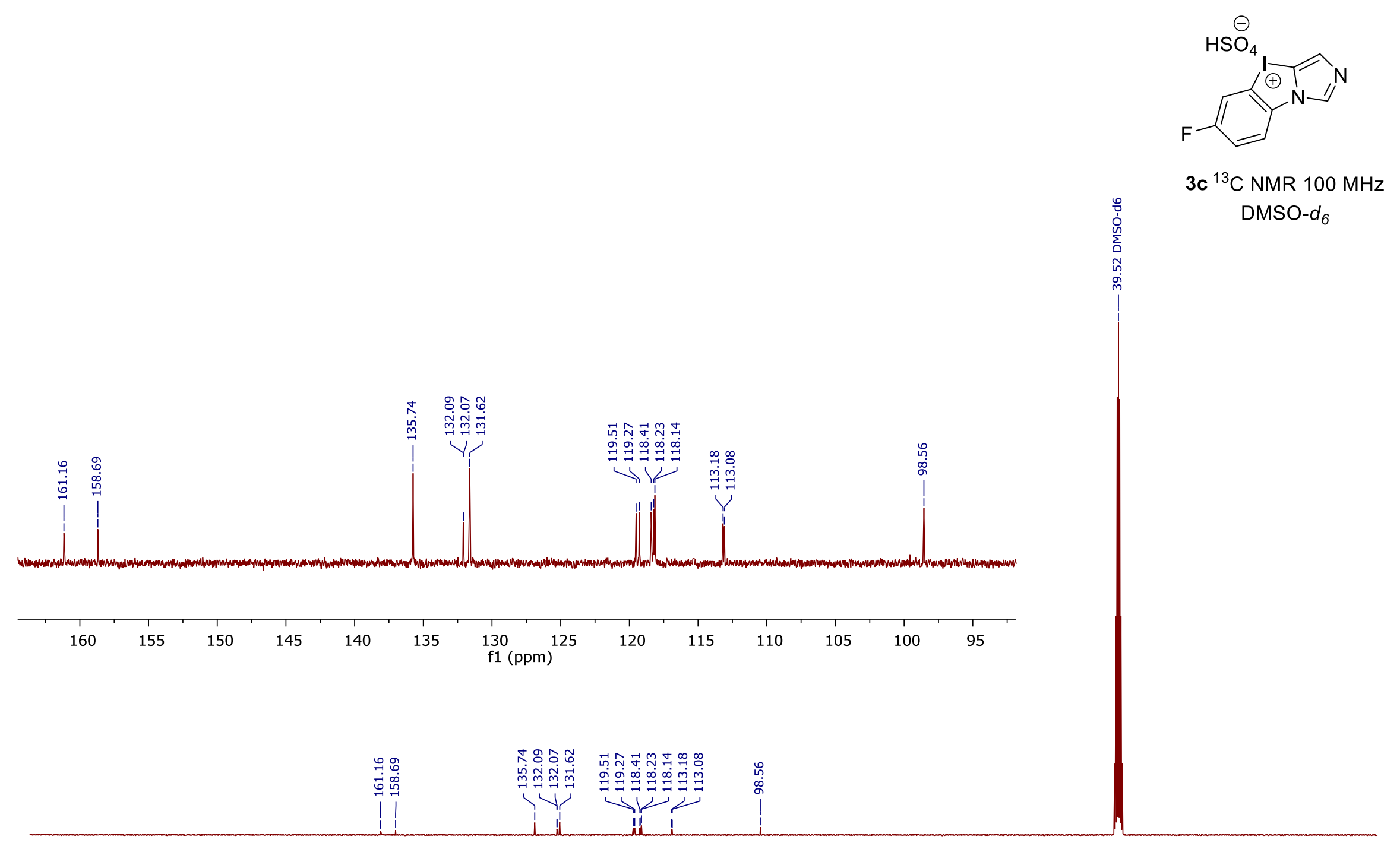

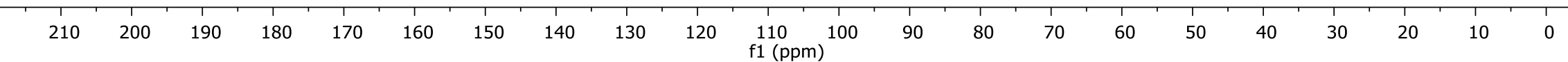



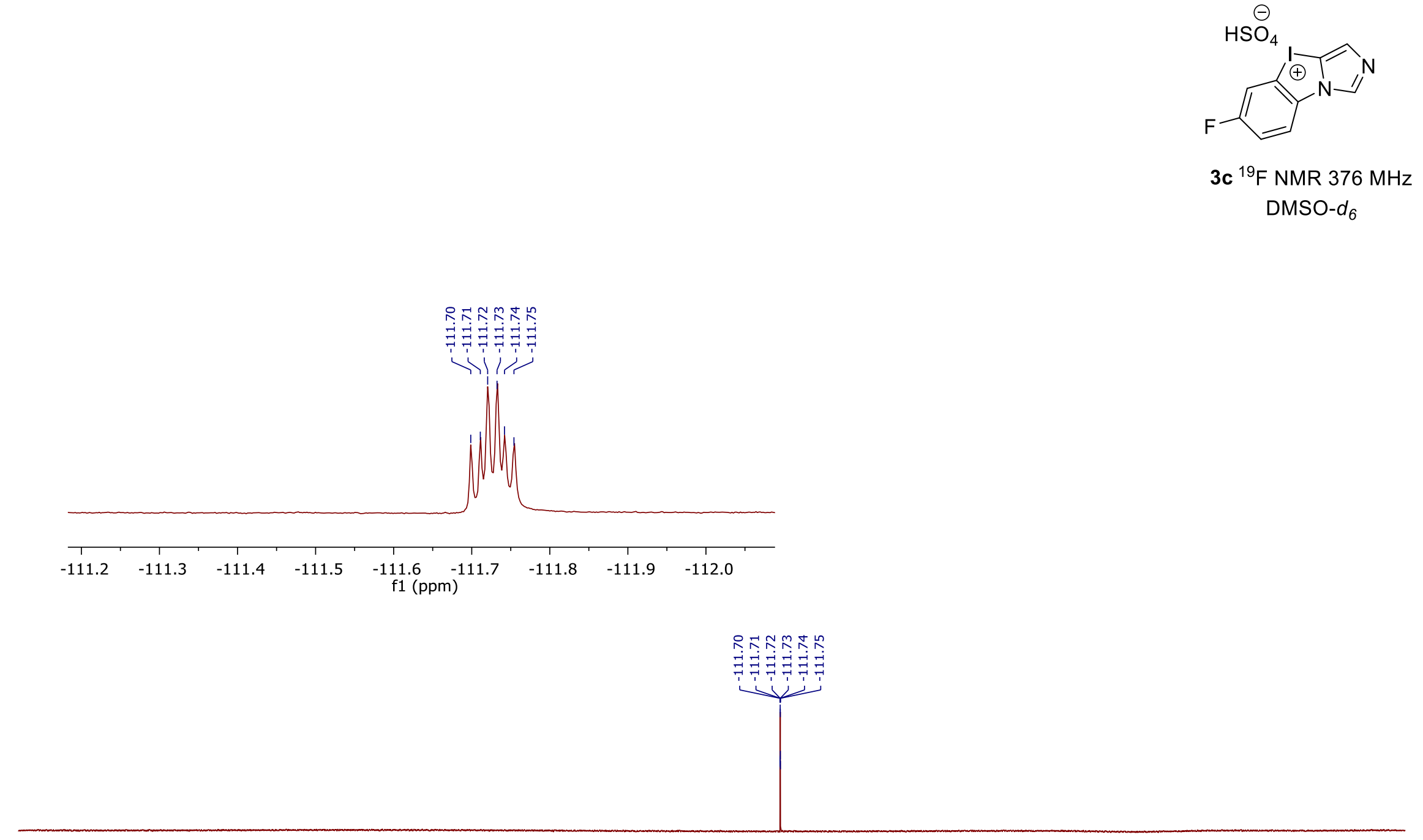

\begin{tabular}{rllllllllllllllllllllllll}
\hline 10 & 0 & -10 & -20 & -30 & -40 & -50 & -60 & -70 & -80 & -90 & -100 & -110 & -120 & -130 & -140 & -150 & -160 & -170 & -180 & -190 & -200 & -210 &
\end{tabular}




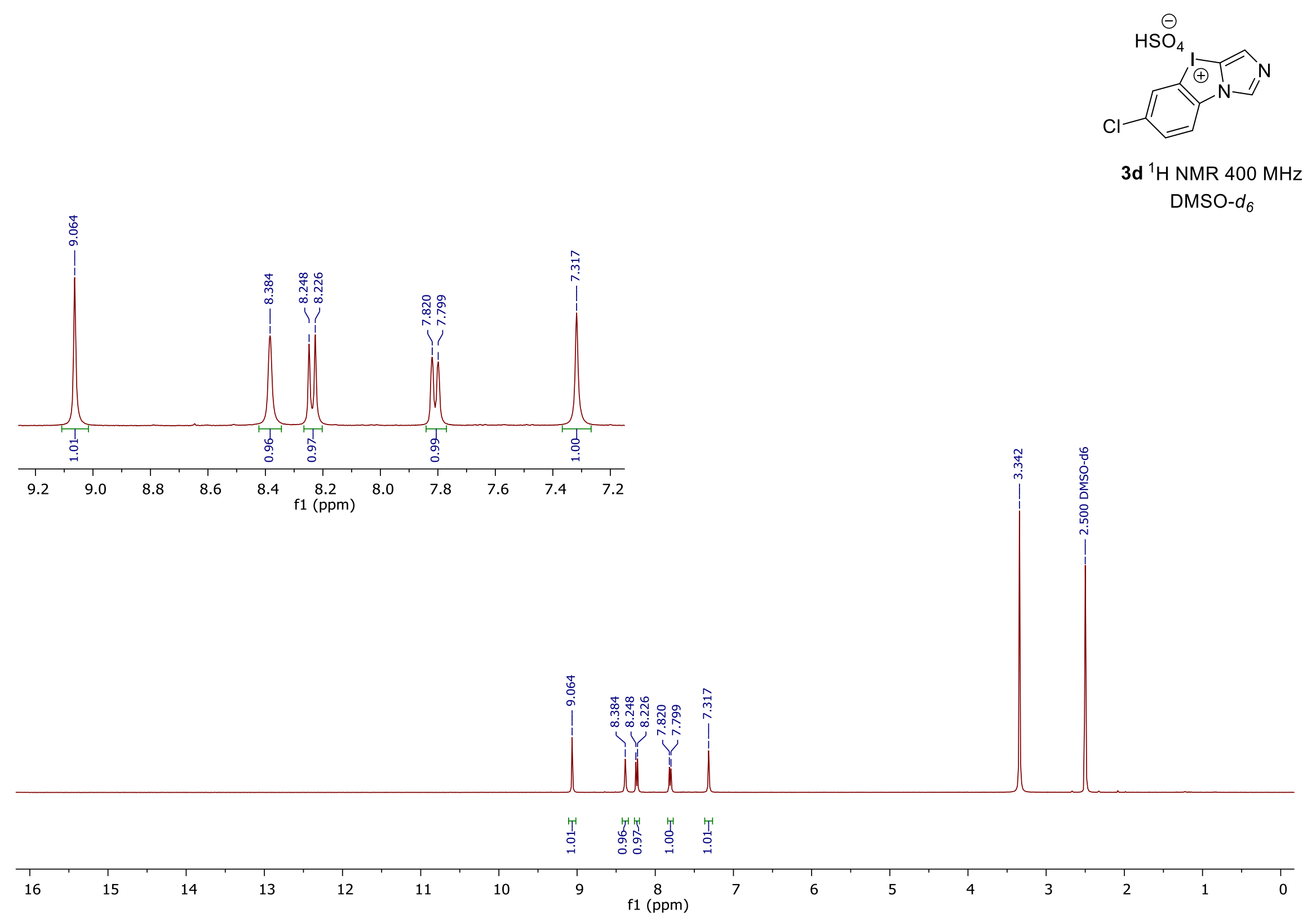




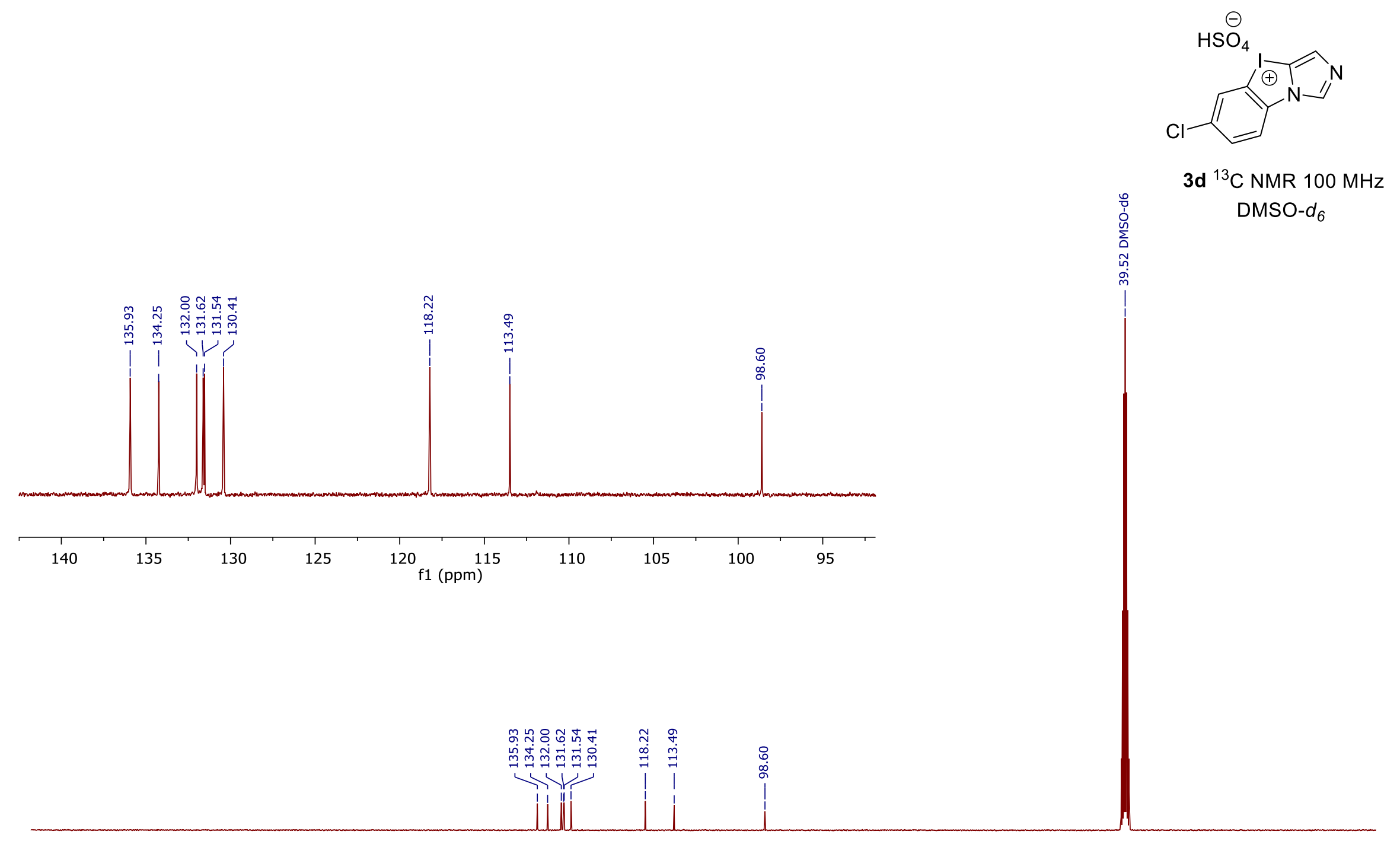

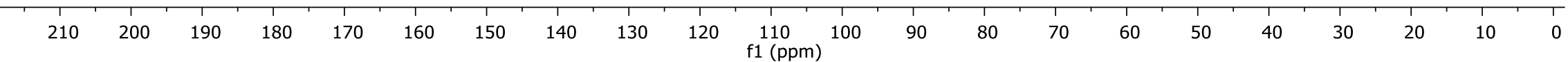


$\ominus$

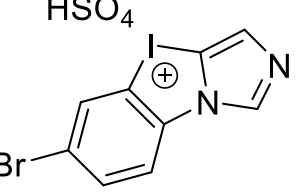

$3 e^{1} \mathrm{H} \mathrm{NMR} 400 \mathrm{MHz}$ DMSO- $d_{6}$

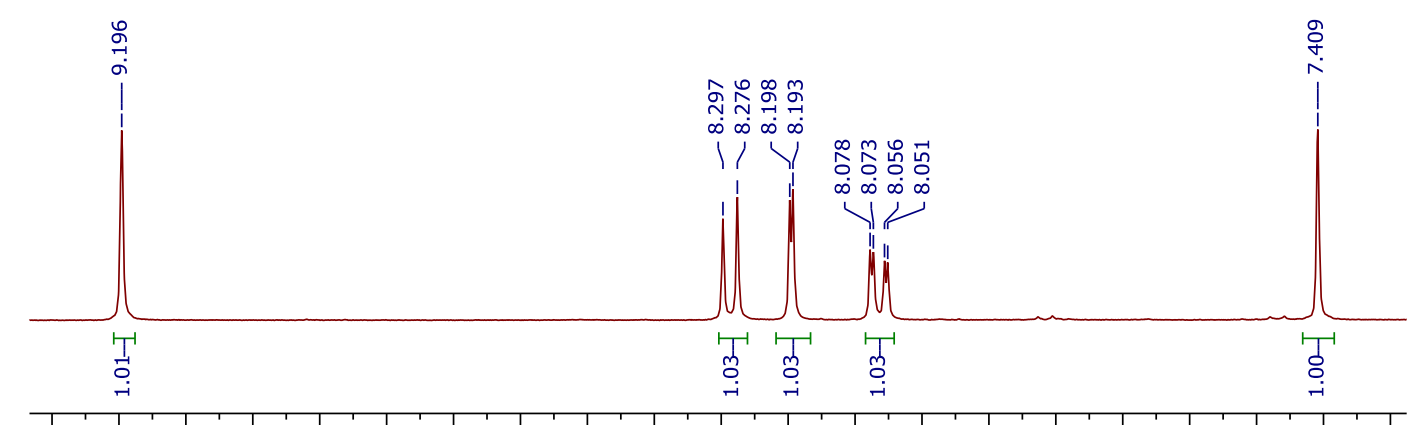

$\begin{array}{llllllllllllllllllllllllll}9.3 & 9.2 & 9.1 & 9.0 & 8.9 & 8.8 & 8.7 & 8.6 & 8.5 & 8.4 & 8 & 3 & 8.2 & 8.1 & 8.0 & 7.9 & 7.8 & 7.7 & 7.6 & 7.5 & 7.4 & 7.3\end{array}$ $\mathrm{f1}(\mathrm{ppm})$

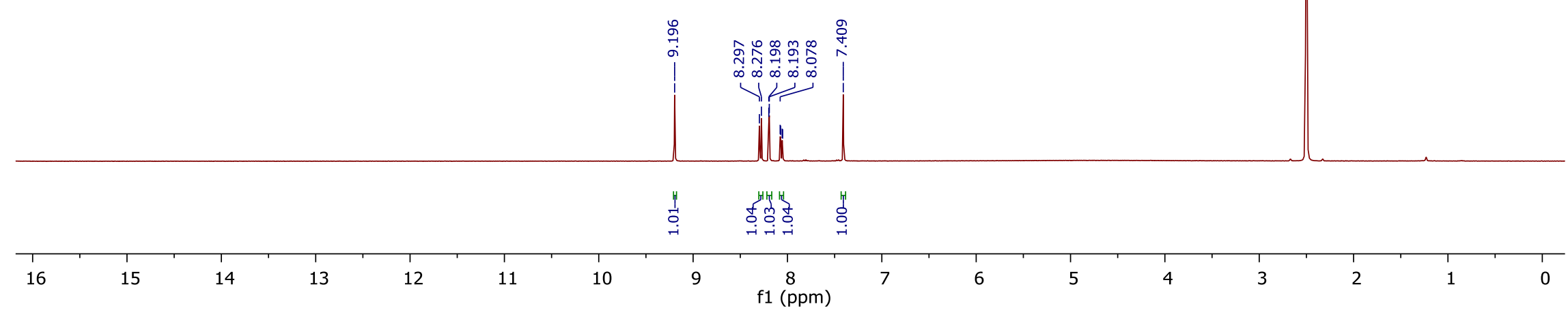




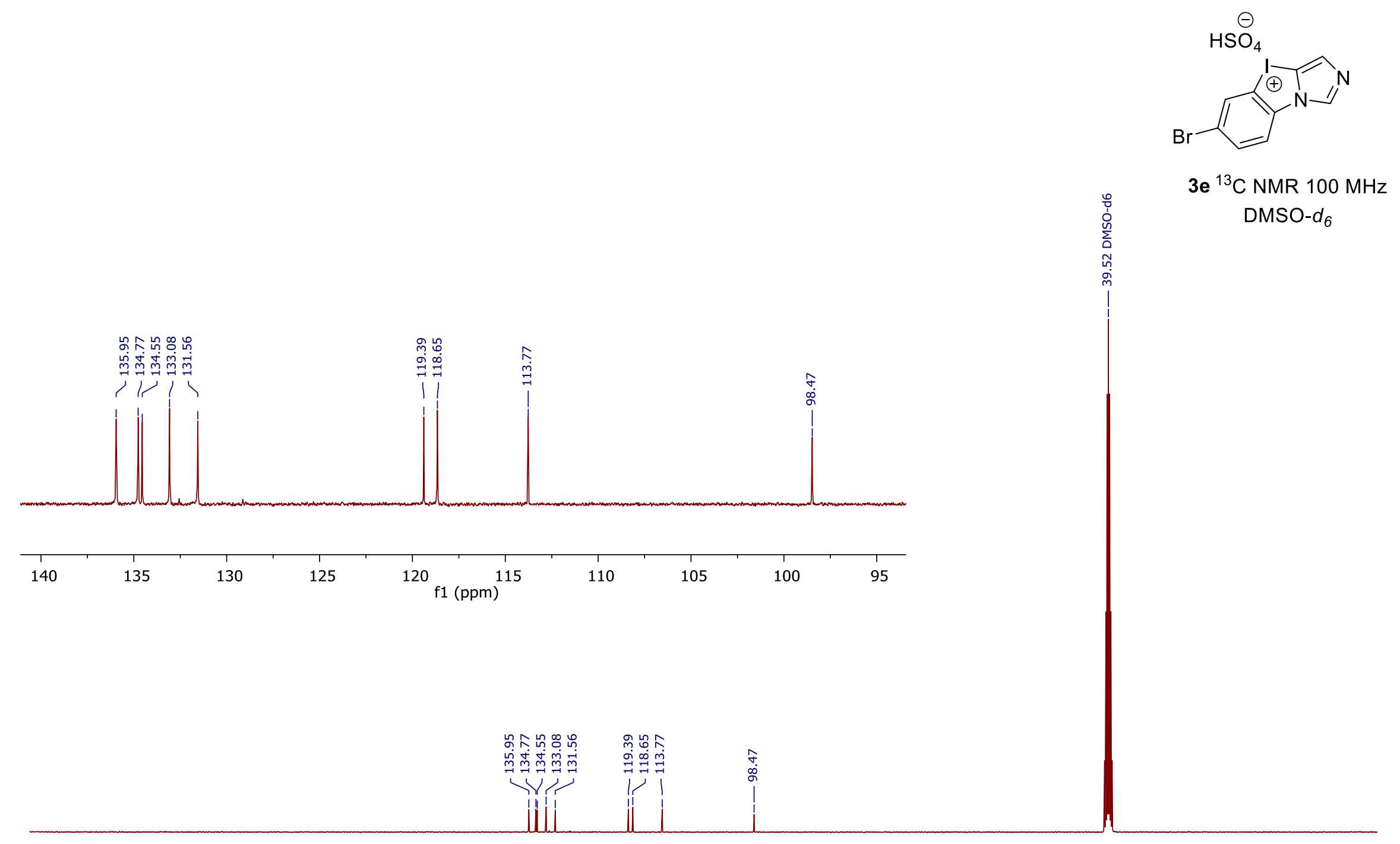

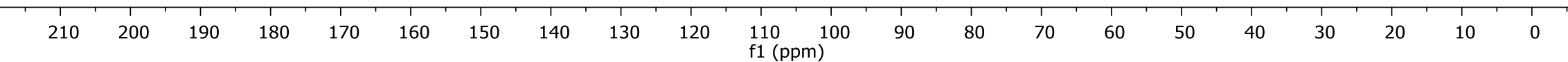



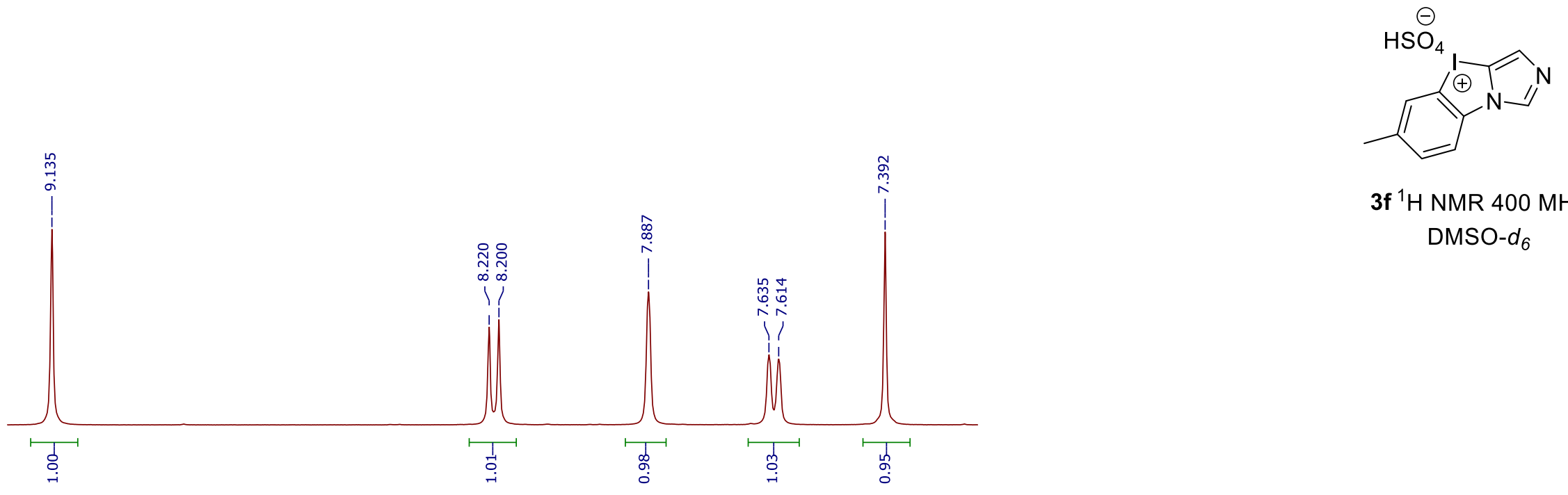

$3 \mathrm{f}^{1} \mathrm{H}$ NMR $400 \mathrm{MHz}$ DMSO- $d_{6}$

$\begin{array}{llllllllllllllllllllll}9.2 & 9.1 & 9.0 & 8.9 & 8.8 & 8.7 & 8.6 & 8.5 & 8.4 & 8.3 & 8.2 & 8.1 & 8.0 & 7.9 & 7.8 & 7.7 & 7.6 & 7.5 & 7.4 & 7.3 & 7.2\end{array}$ f1 (ppm)

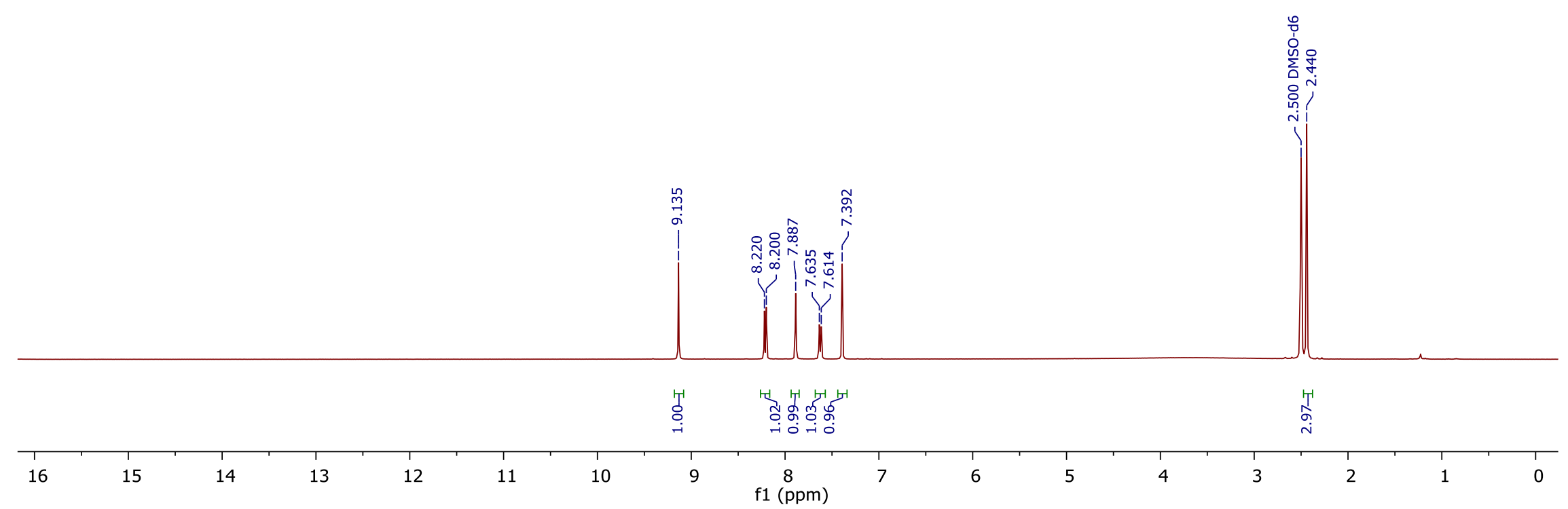




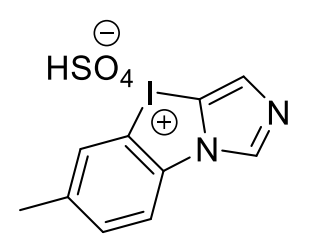

$3 f{ }^{13} \mathrm{C}$ NMR $100 \mathrm{MHz}$ DMSO-d 6

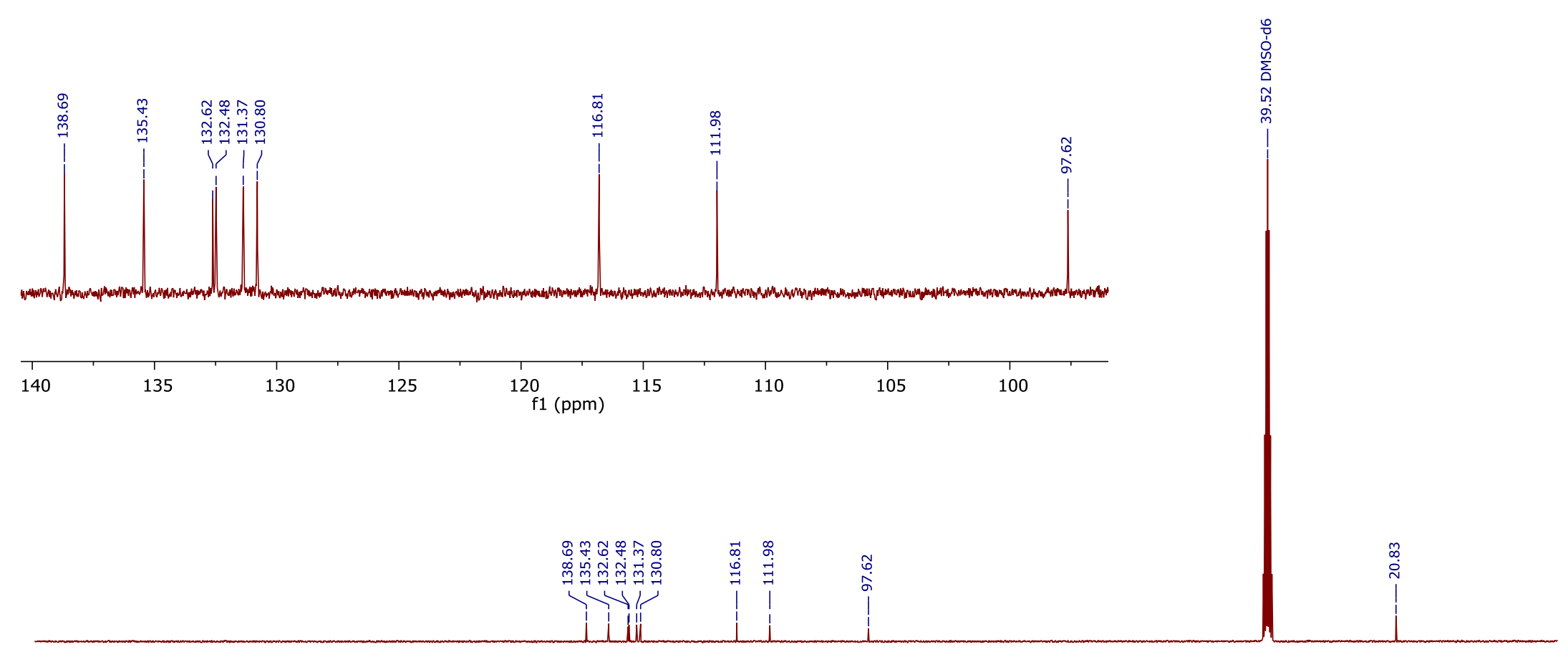

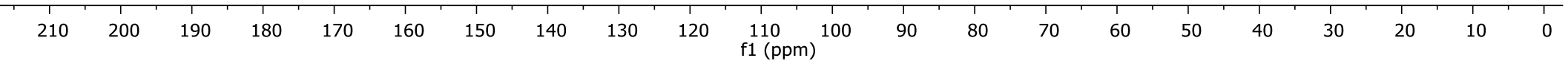




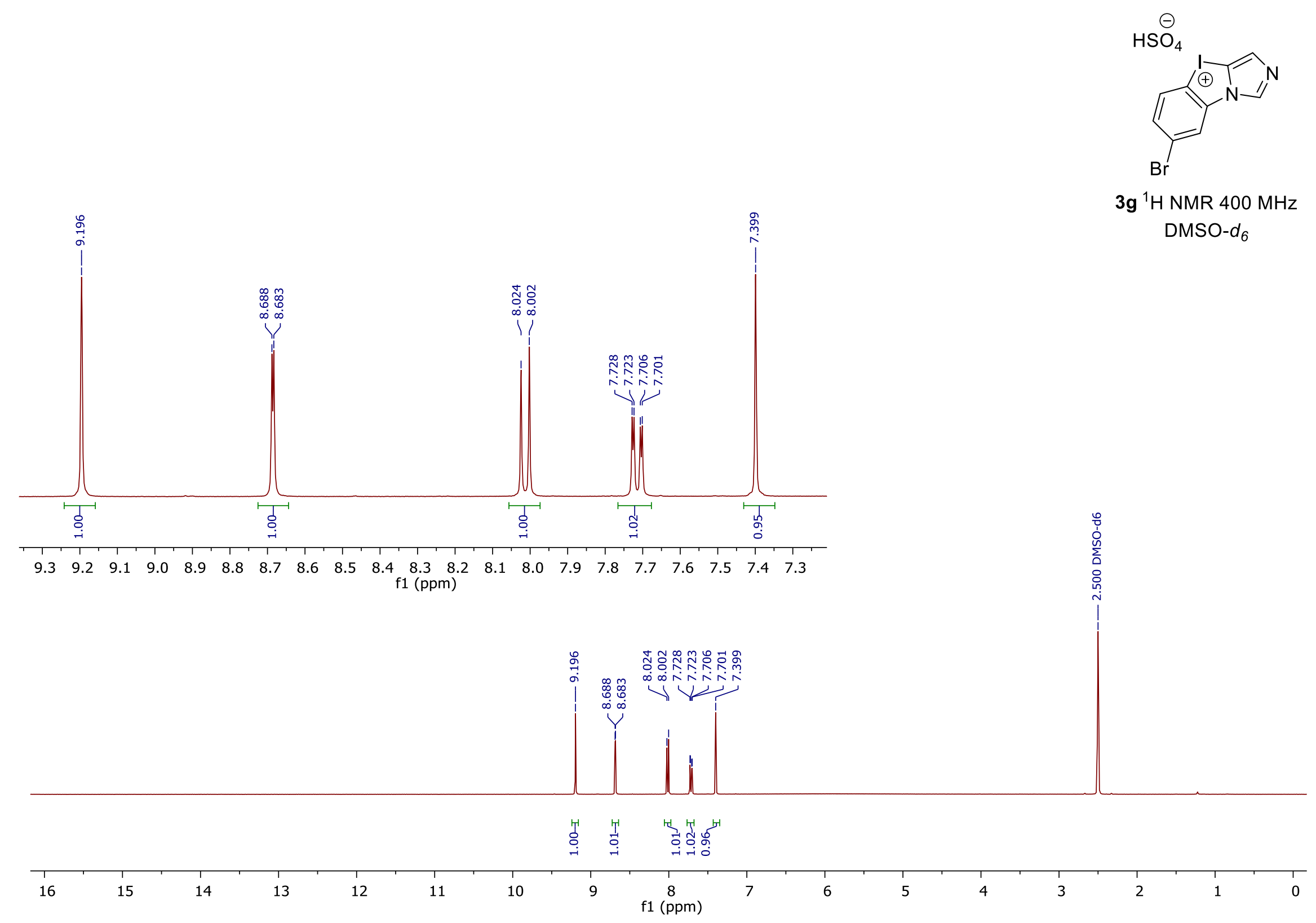




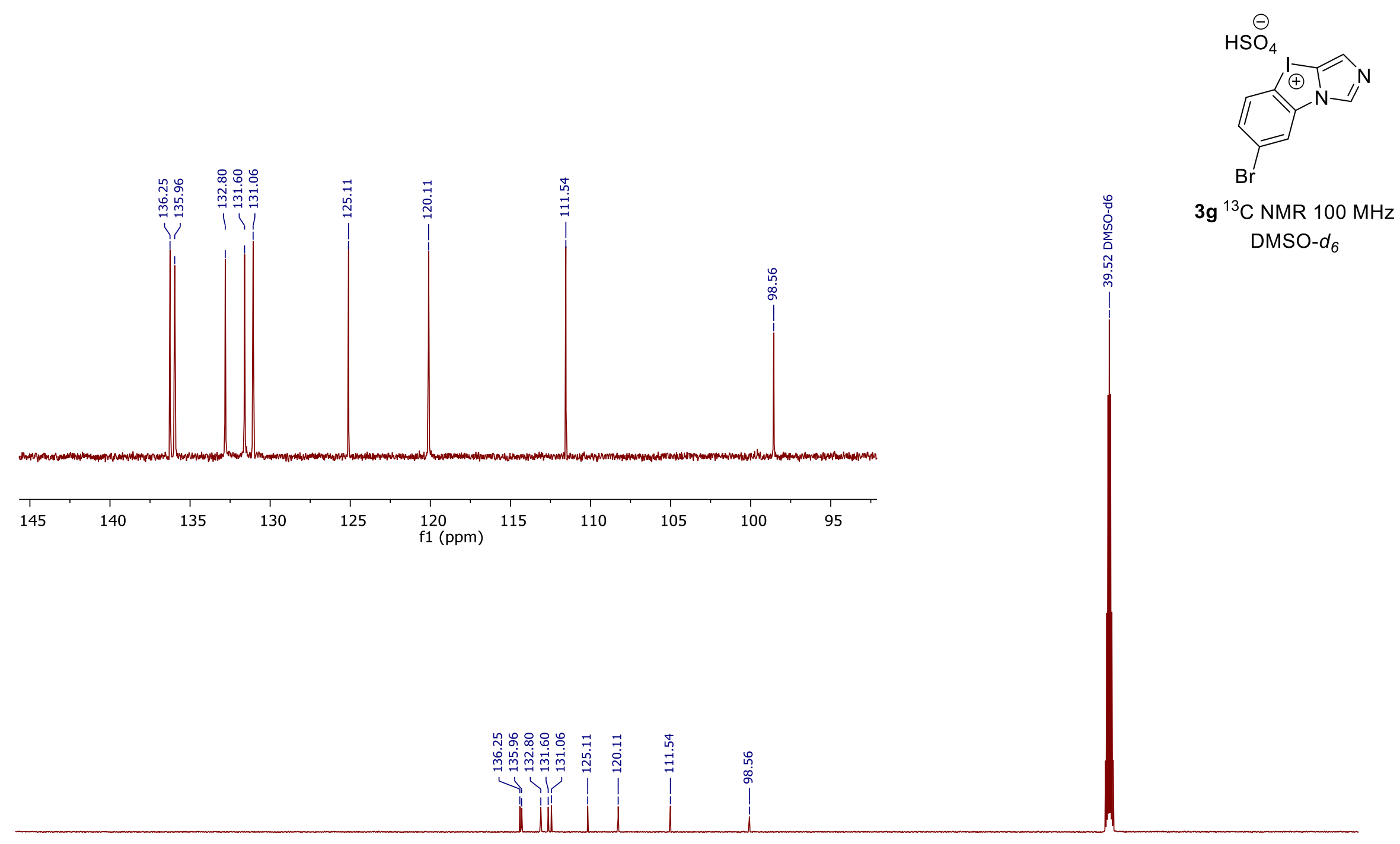

\begin{tabular}{lllllllllllllllllllllll}
\hline & 1 \\
210 & 200 & 190 & 180 & 170 & 160 & 150 & 140 & 130 & 120 & 110 & 100 & 90 & 80 & 70 & 60 & 50 & 40 & 30 & 20 & 10 & 0
\end{tabular}




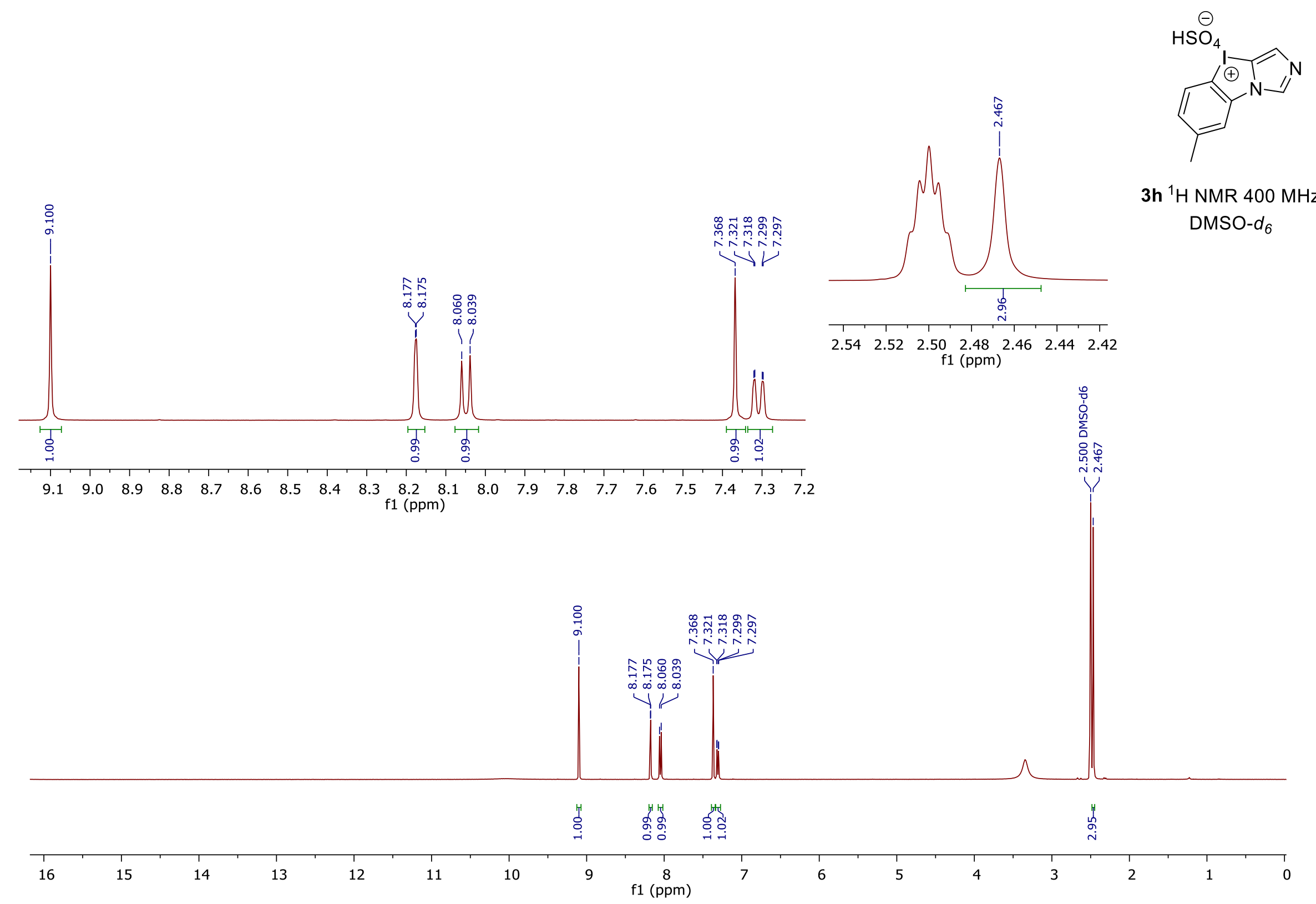




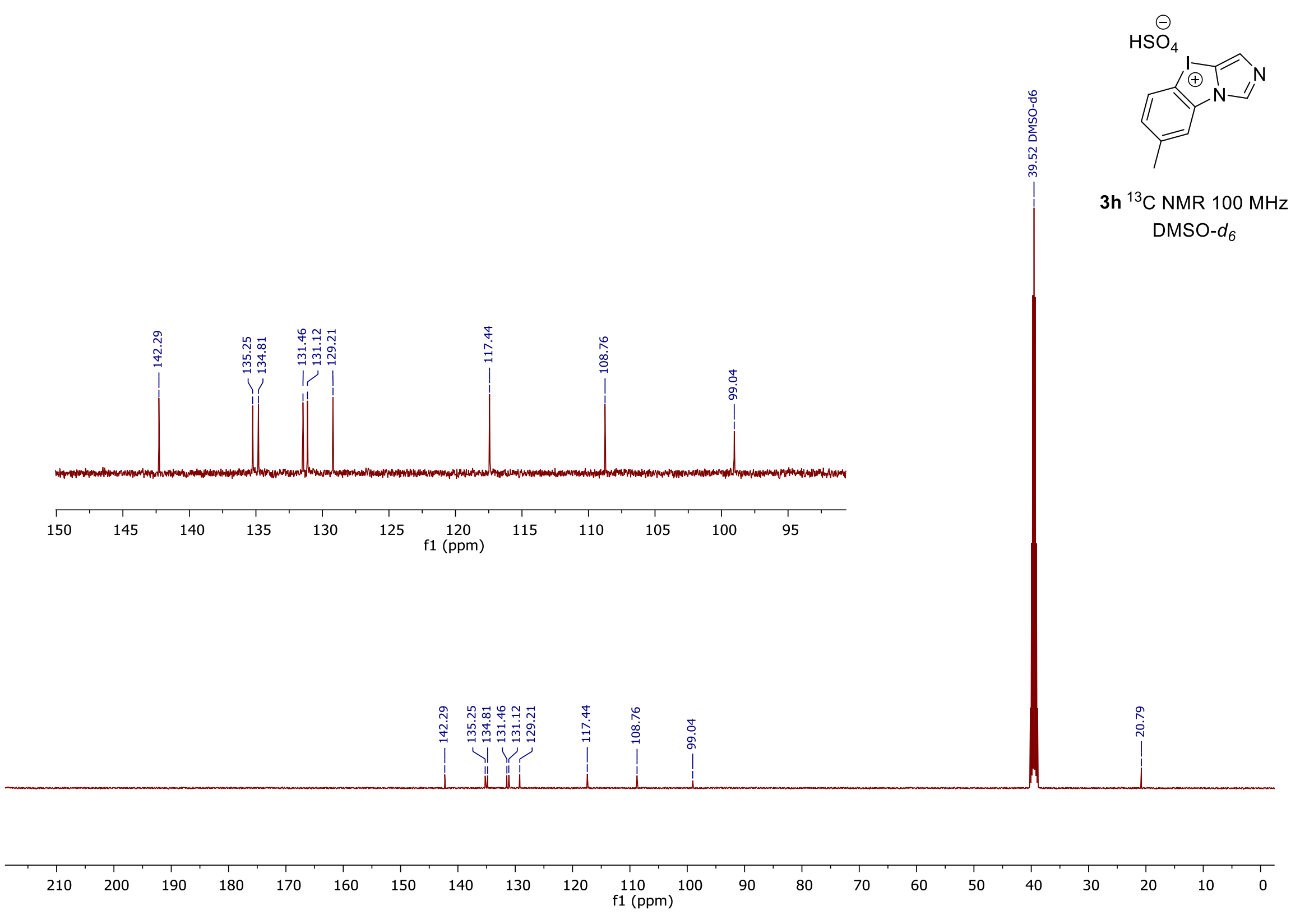




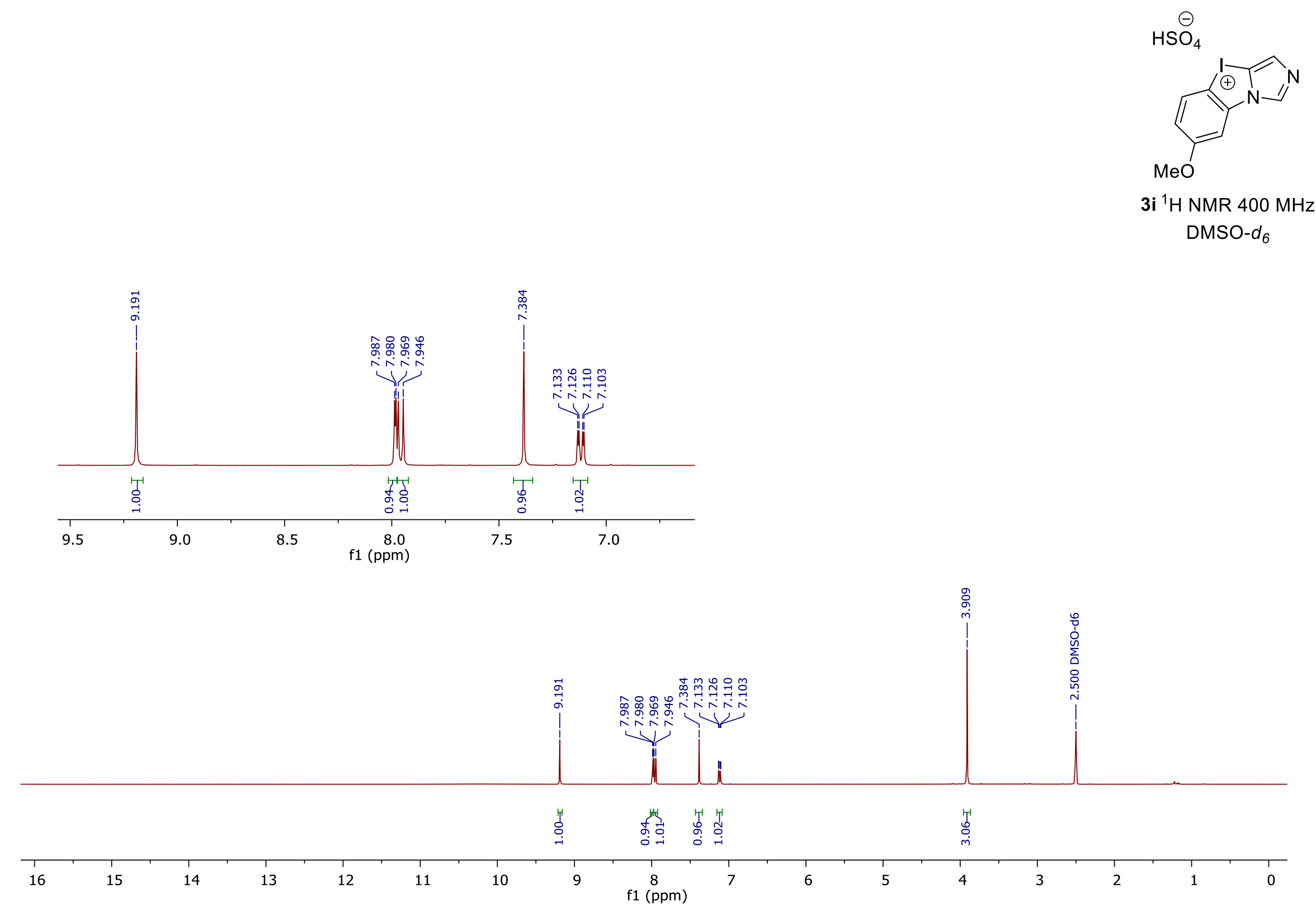




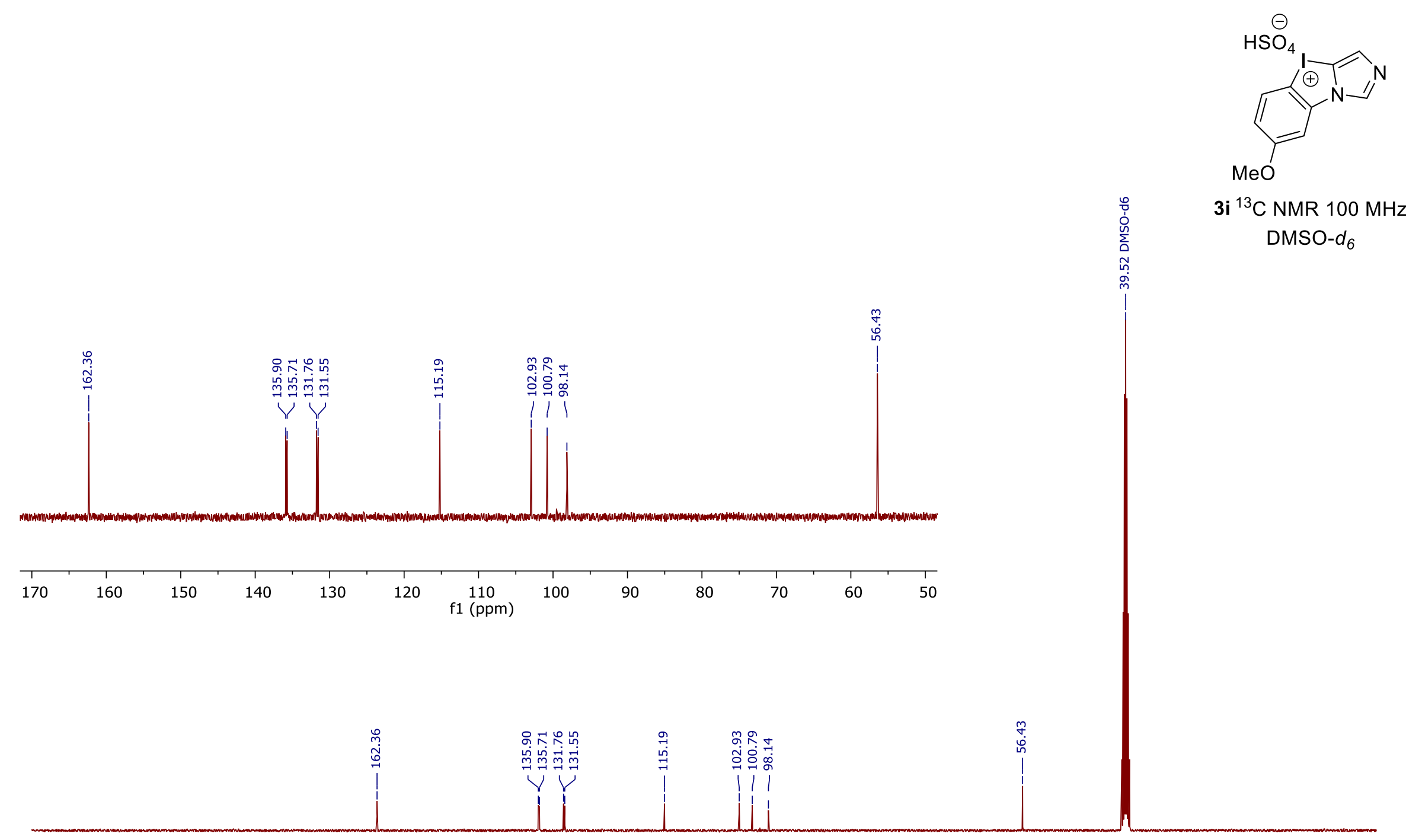

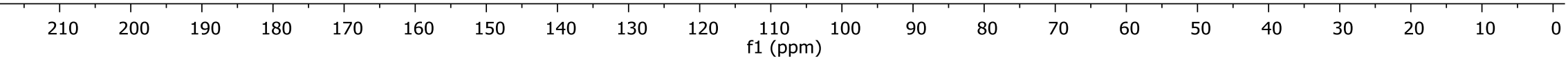


$\mathrm{HSO}_{4}^{\ominus}$

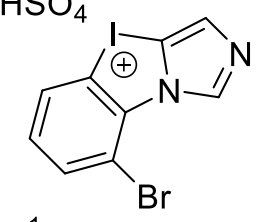

3j ${ }^{1} \mathrm{H}$ NMR $400 \mathrm{MHz}$ DMSO- $d_{6}$
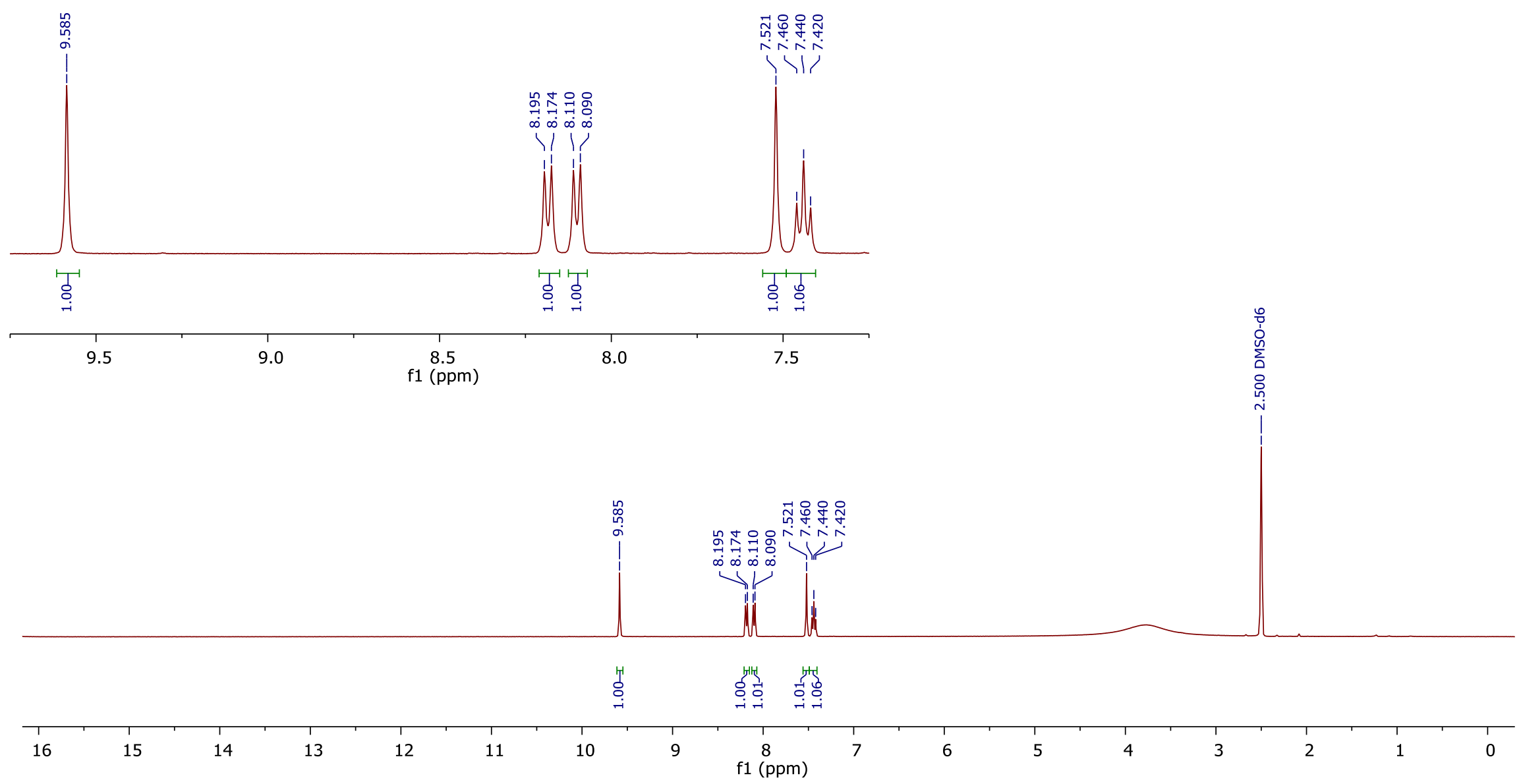


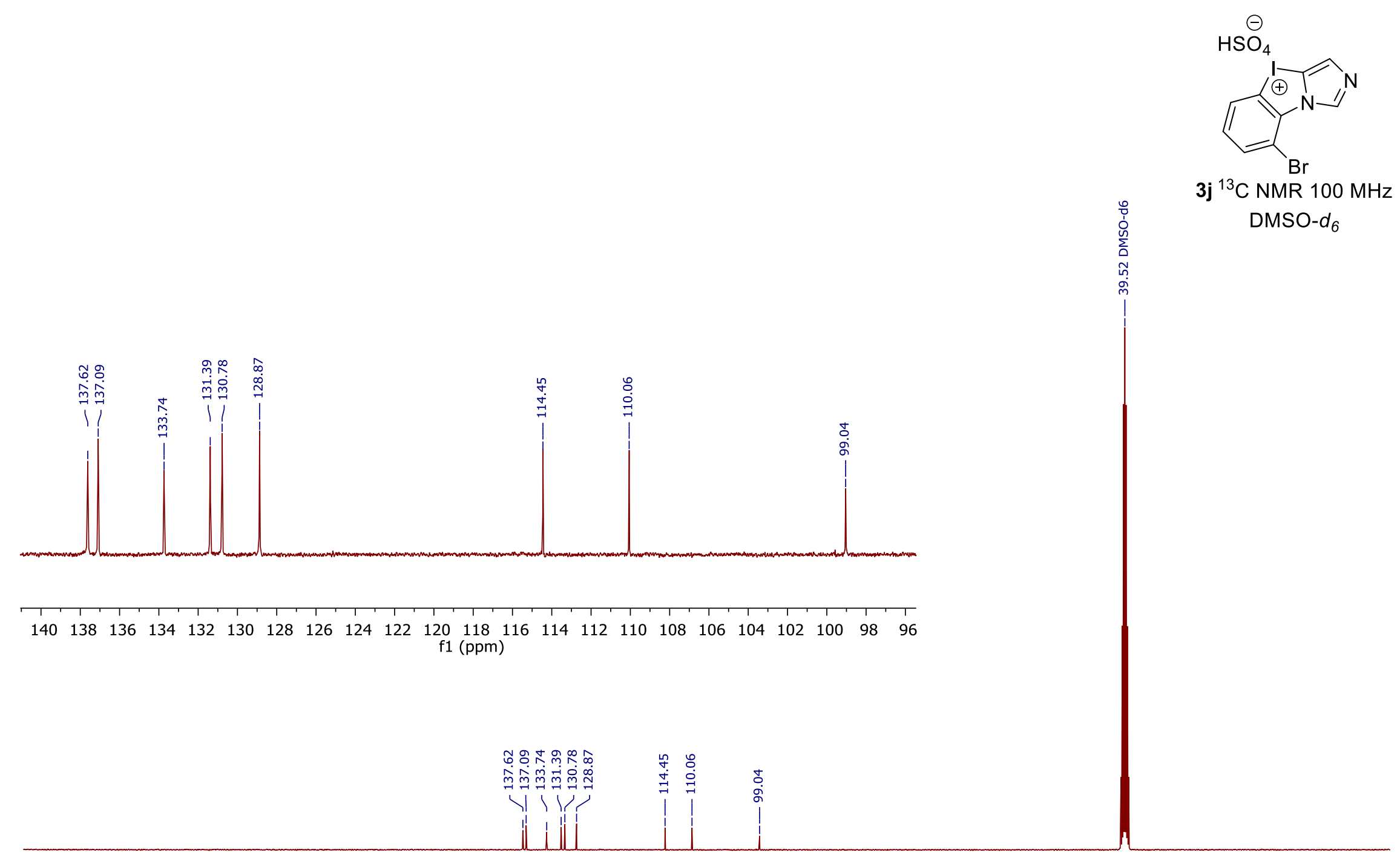

\begin{tabular}{llllllllllllllllllllllllll}
\hline 210 & 200 & 190 & 180 & 170 & 160 & 150 & 140 & 130 & 120 & 110 & 100 & 90 & 80 & 70 & 60 & 50 & 40 & 30 & 20 & 10 & 0
\end{tabular}




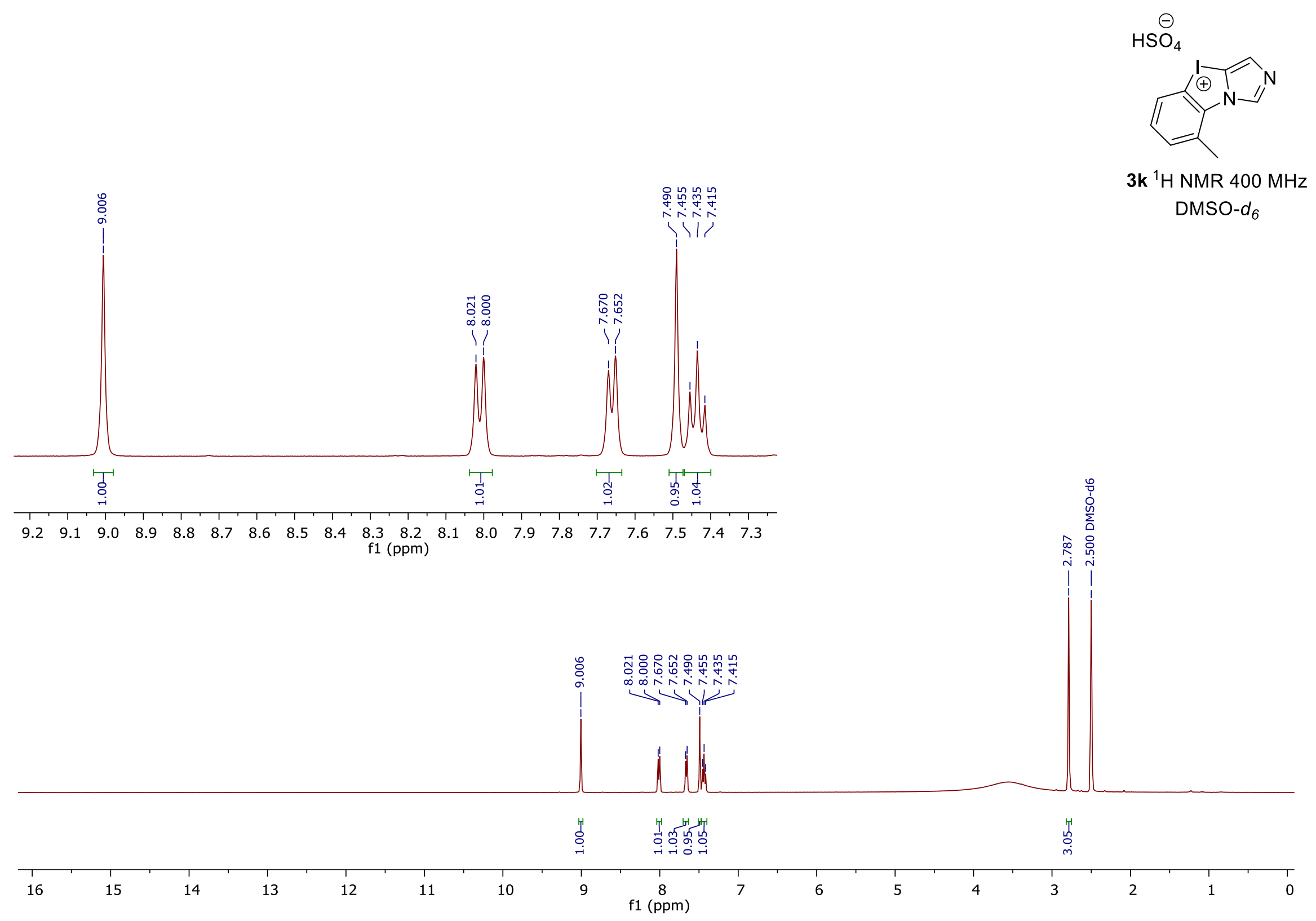




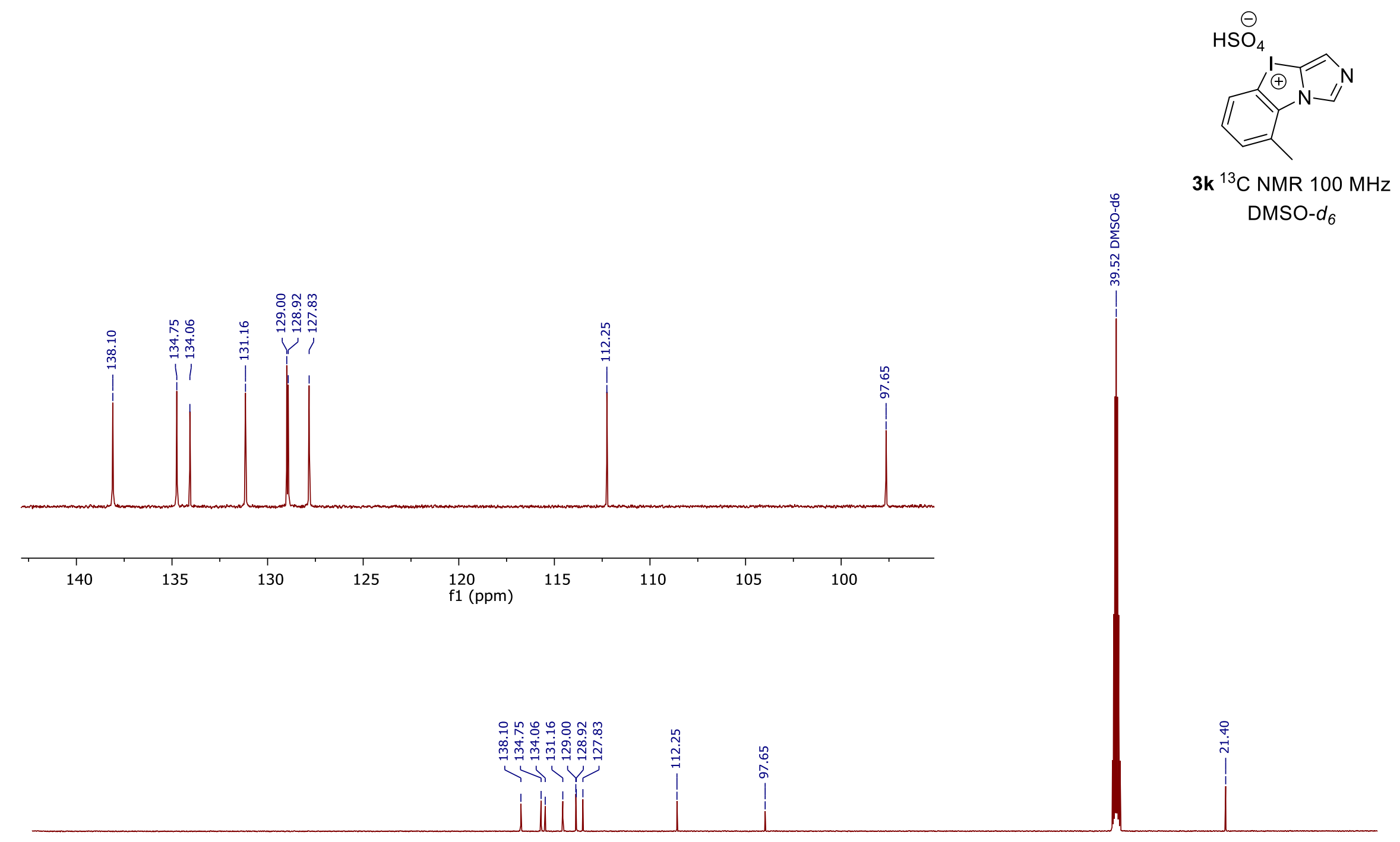

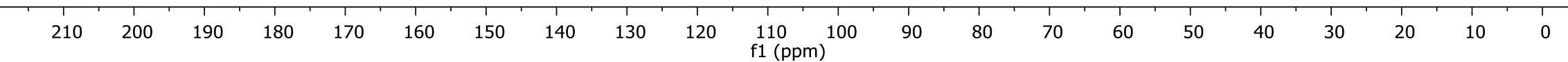




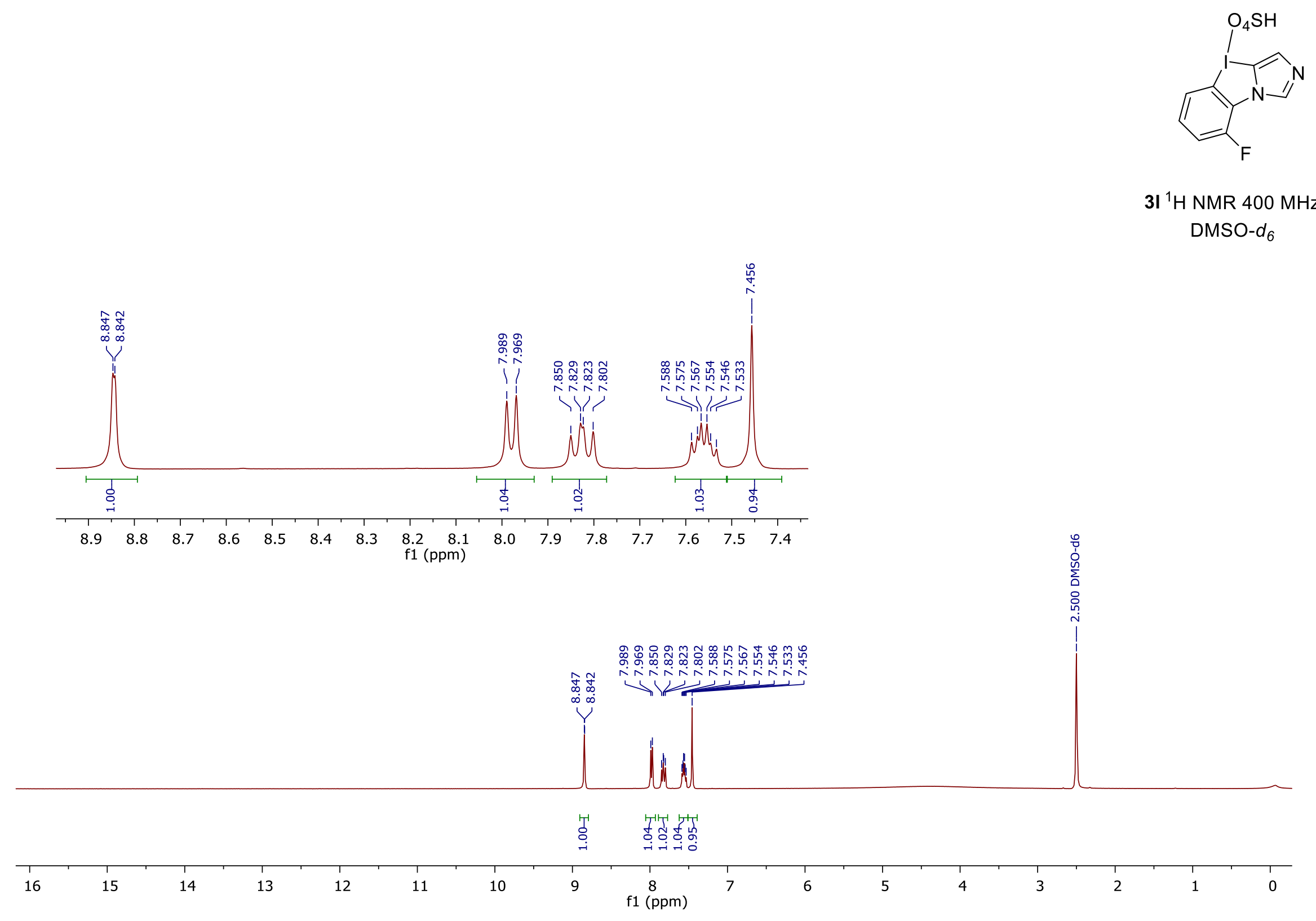




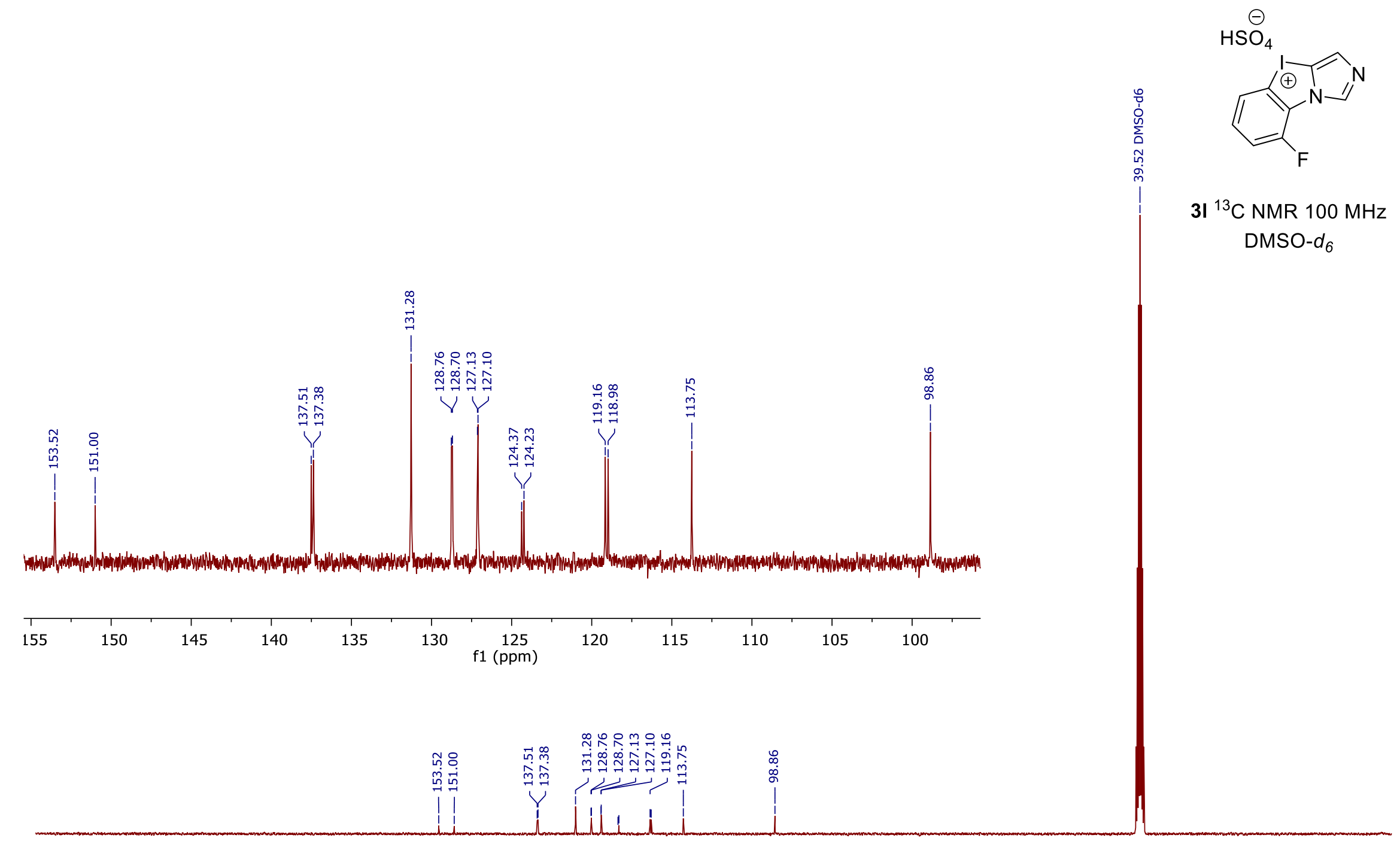

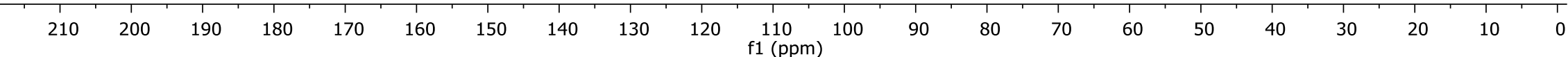


$\Theta$

$\mathrm{HSO}_{4}$

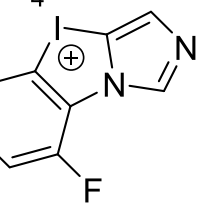

3I ${ }^{19} \mathrm{~F}$ NMR $376 \mathrm{MHz}$ DMSO- $d_{6}$

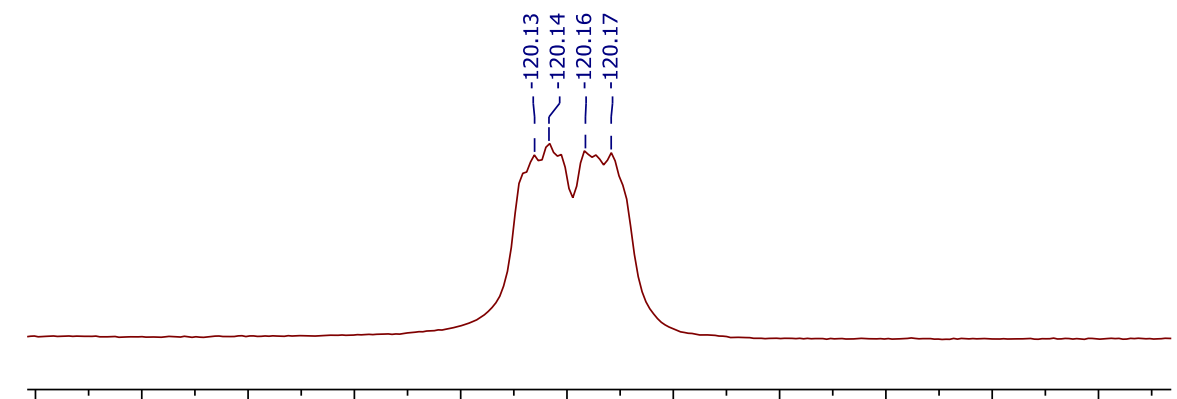

$-119.90-119.95-120.00-120.05-120.10-120.15-120.20-120.25-120.30-120.35-120.40$ f1 (ppm)

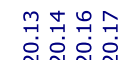

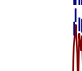

\begin{tabular}{lllllllllllllllllllllllllll}
\hline-113.0 & -113.5 & -114.0 & -114.5 & -115.0 & -115.5 & -116.0 & -116.5 & -117.0 & -117.5 & -118.0 & -118.5 & -119.0 & -119.5 & -120.0 & -120.5 & -121.0 & -121.5 & -122.0 & -122.5 & -123.0
\end{tabular} 
$\stackrel{\ominus}{\mathrm{HSO}_{4}}$

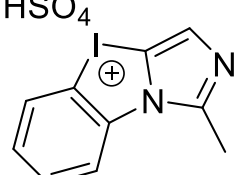

$3 \mathrm{~m}^{1} \mathrm{H}$ NMR $400 \mathrm{MHz}$ DMSO- $d_{6}$
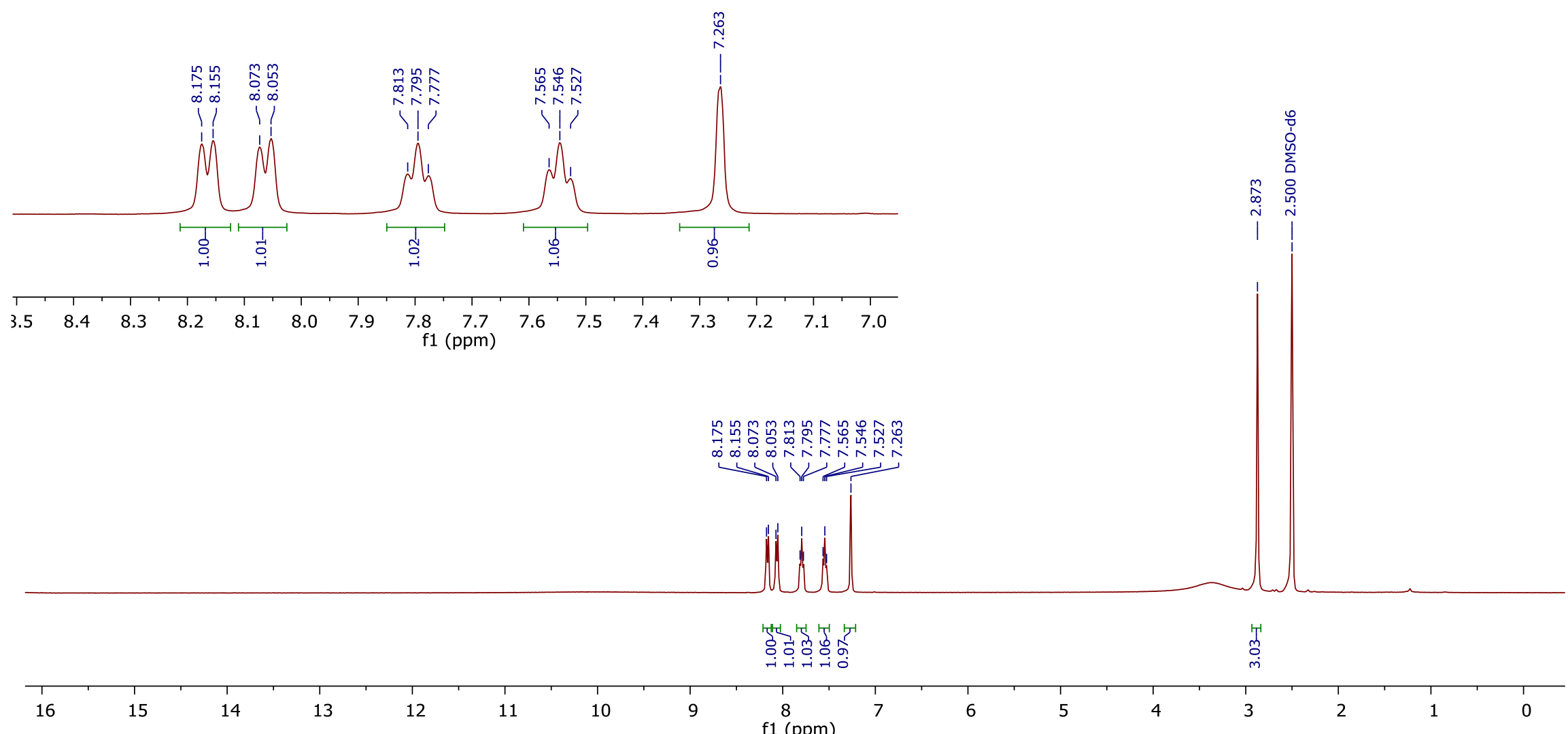
$\ominus$

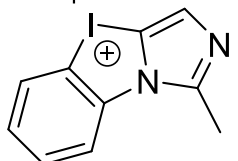

$3 \mathrm{~m}{ }^{13} \mathrm{C}$ NMR $100 \mathrm{MHz}$ DMSO- $d_{6}$
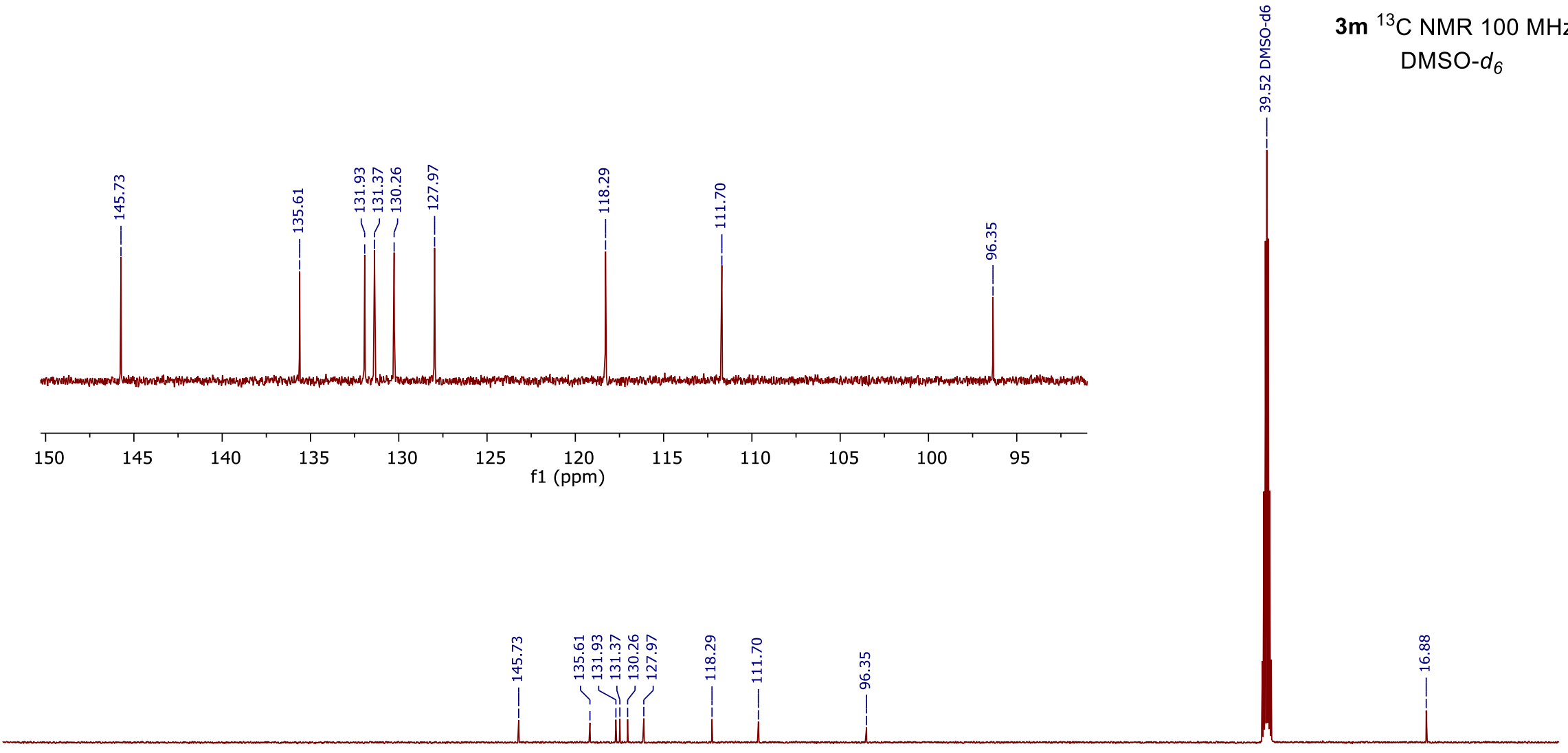

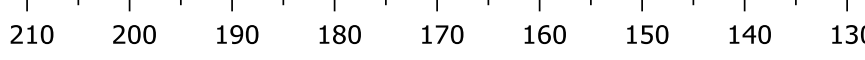

$120 \quad 110$

$\begin{array}{ll}110 & 100\end{array}$

90

$80 \quad 70$

60

$50 \quad 40$

$40 \quad 30$

20

$10 \quad 0$ 


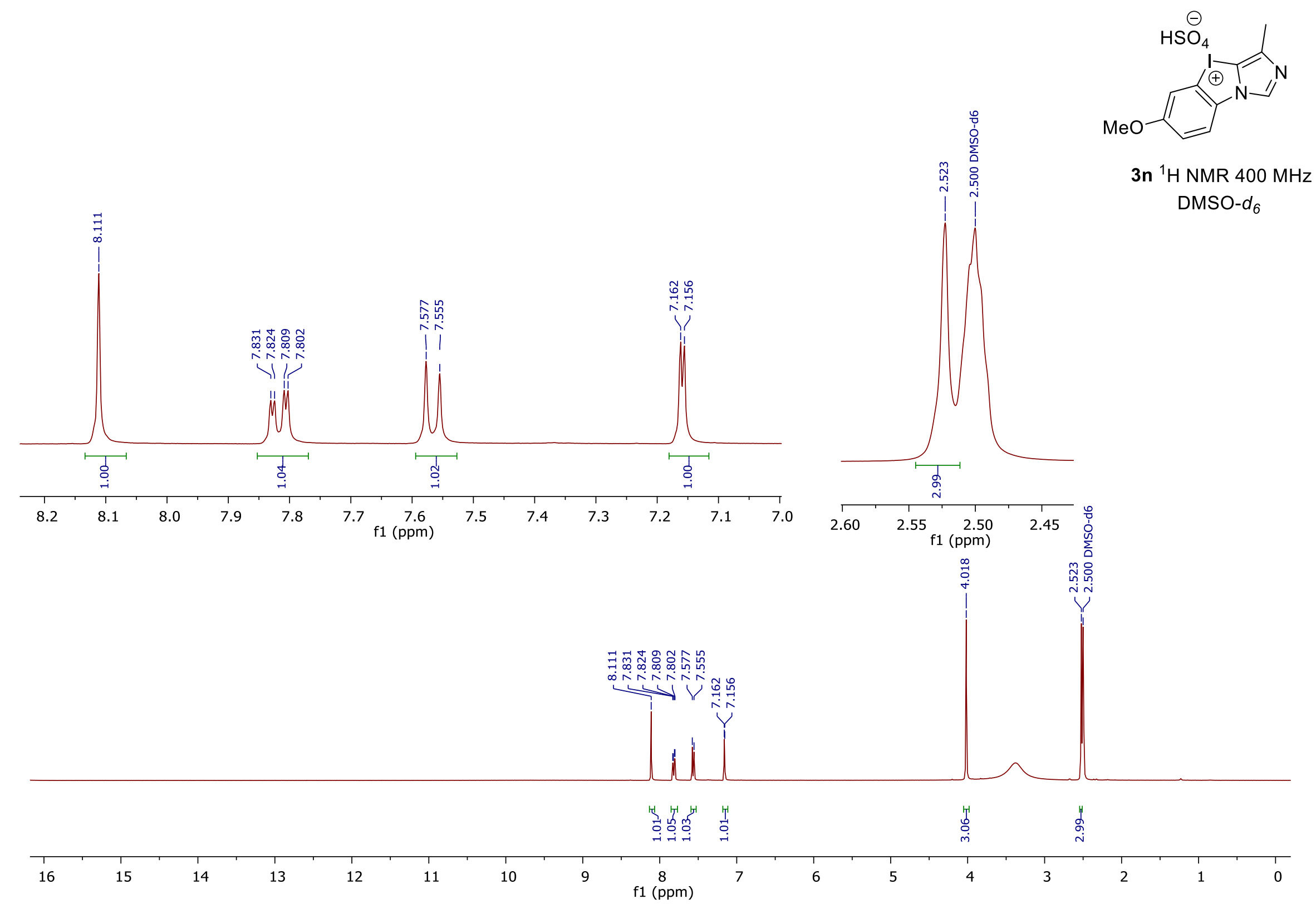




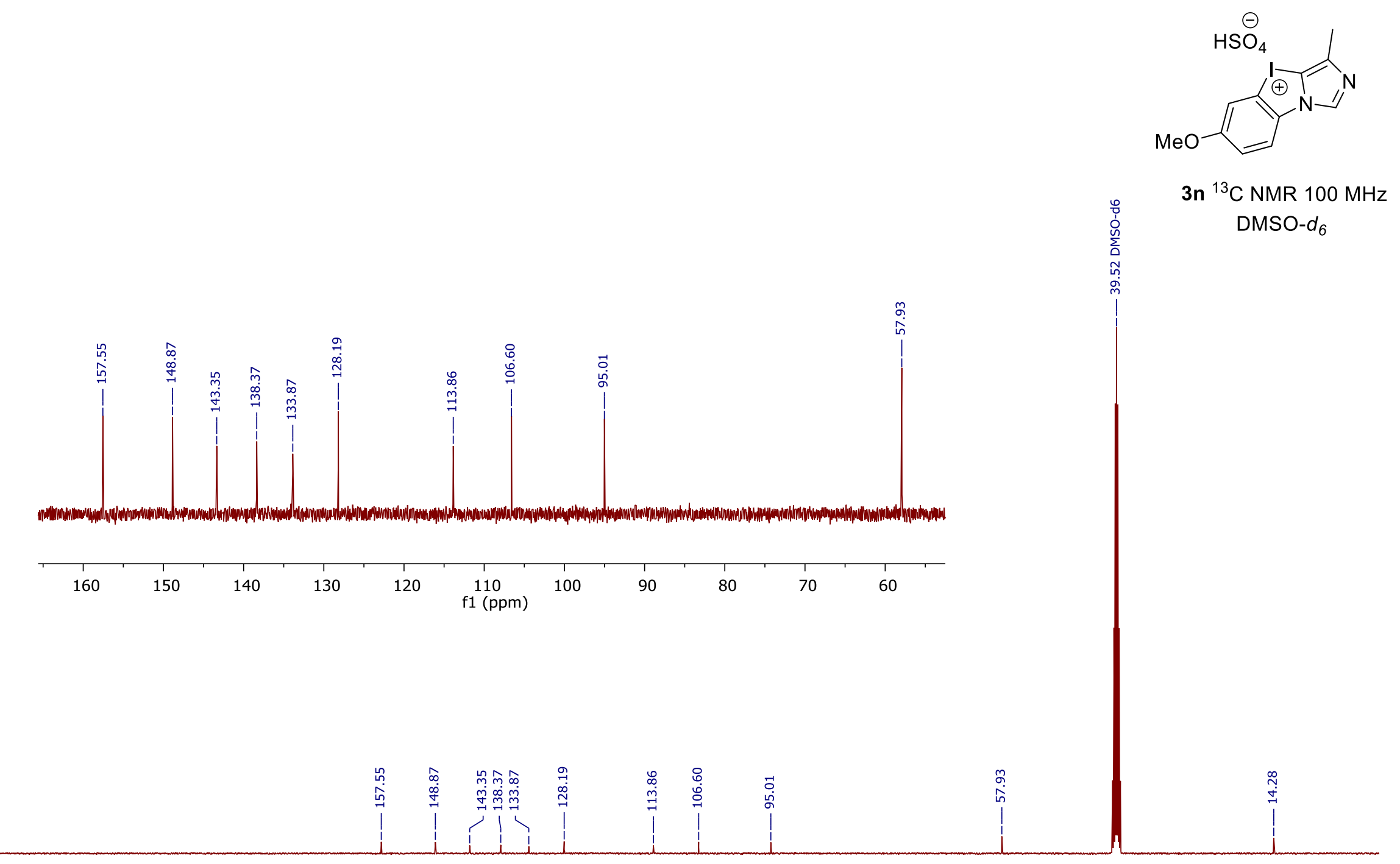

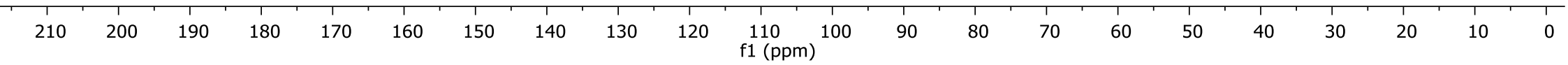




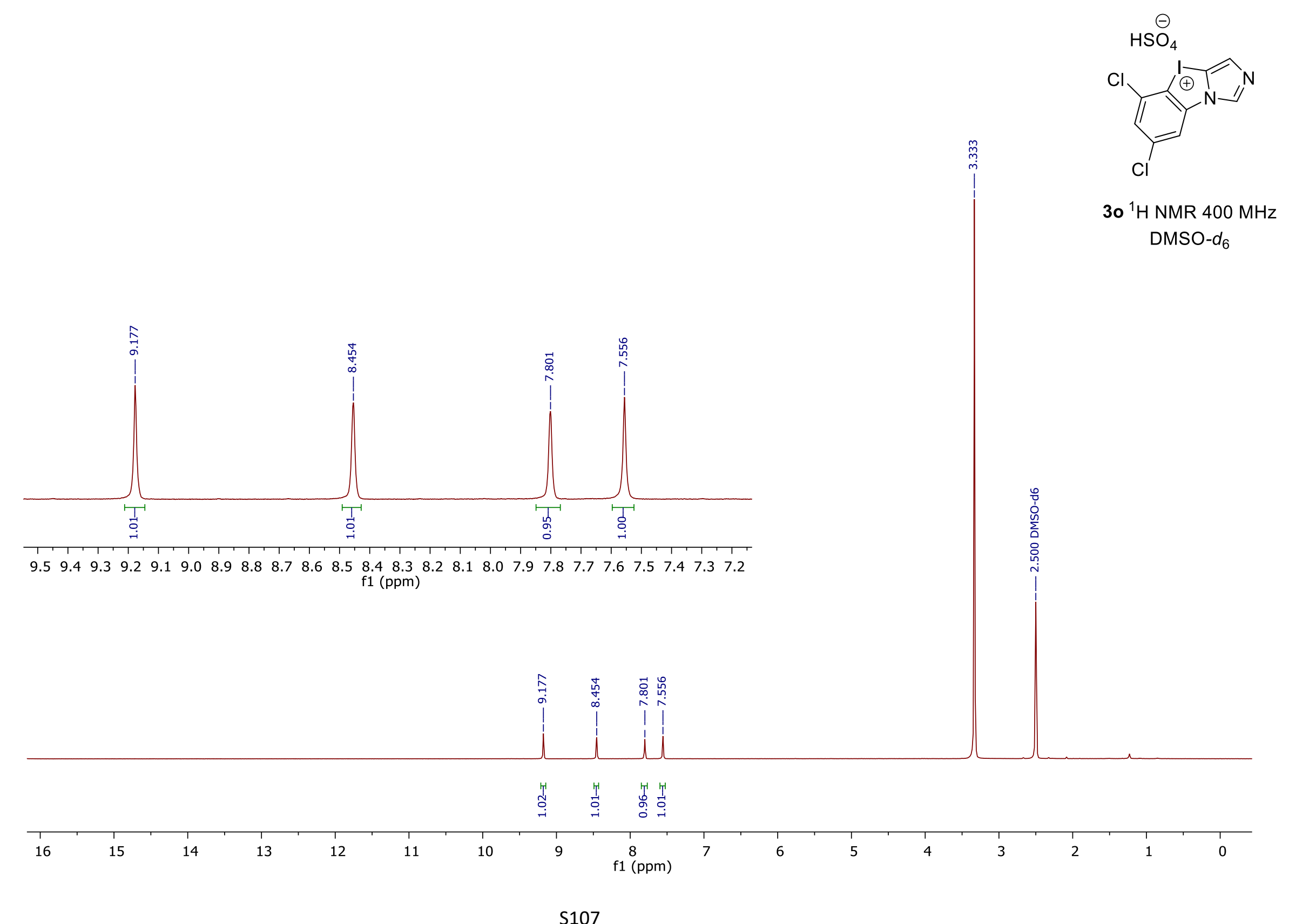




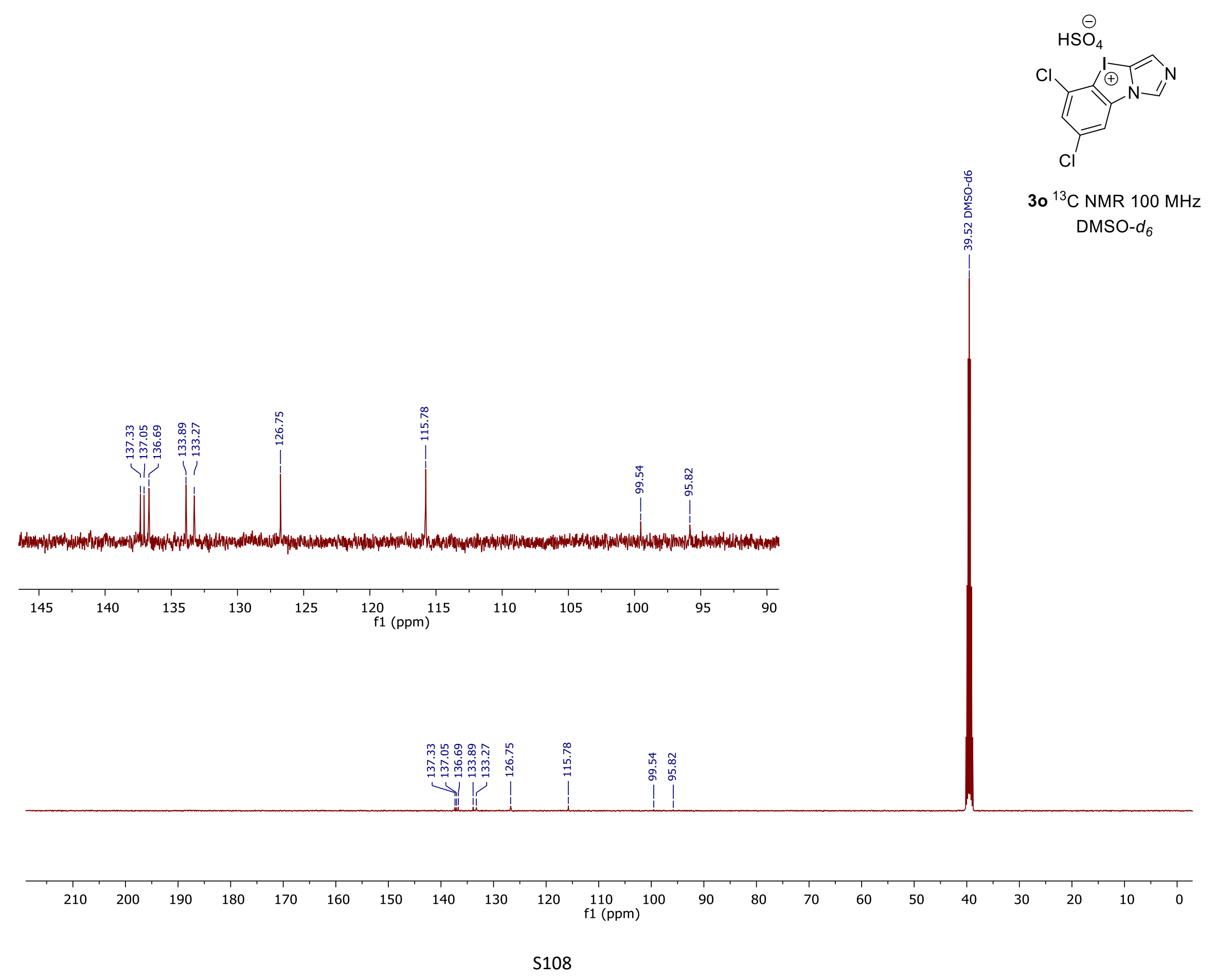




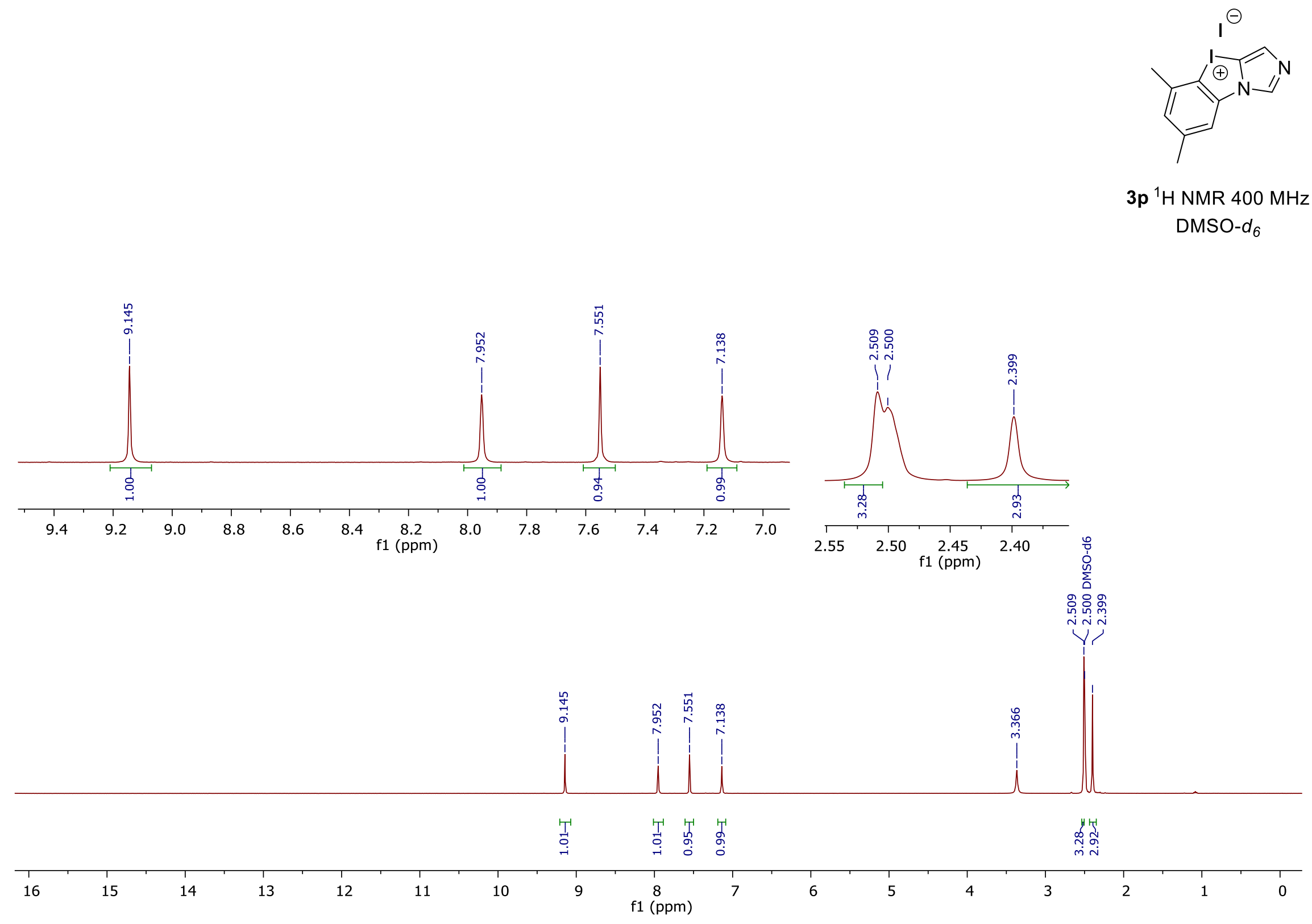




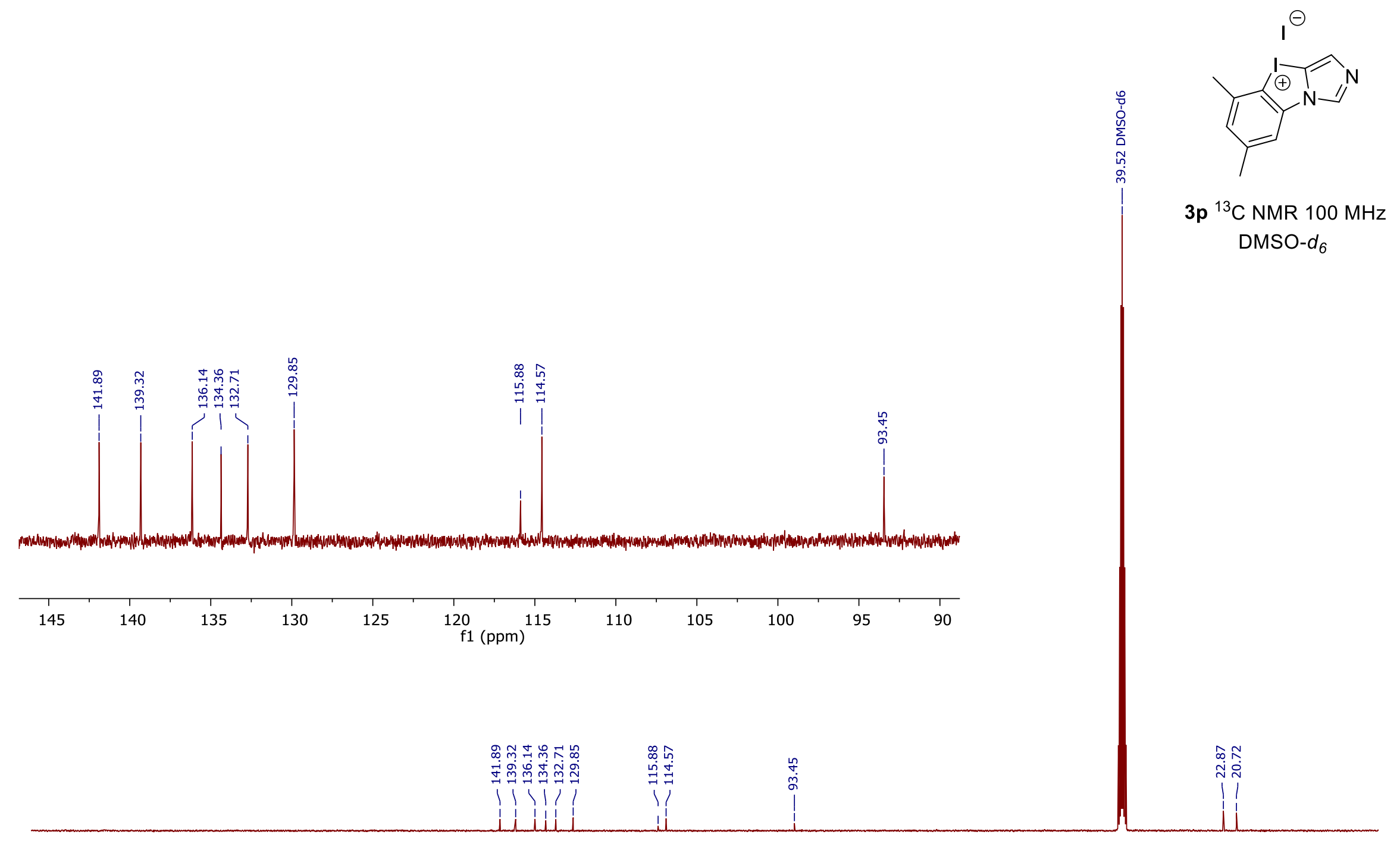

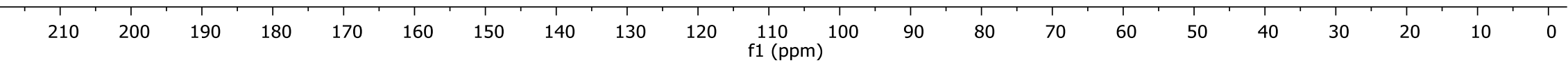



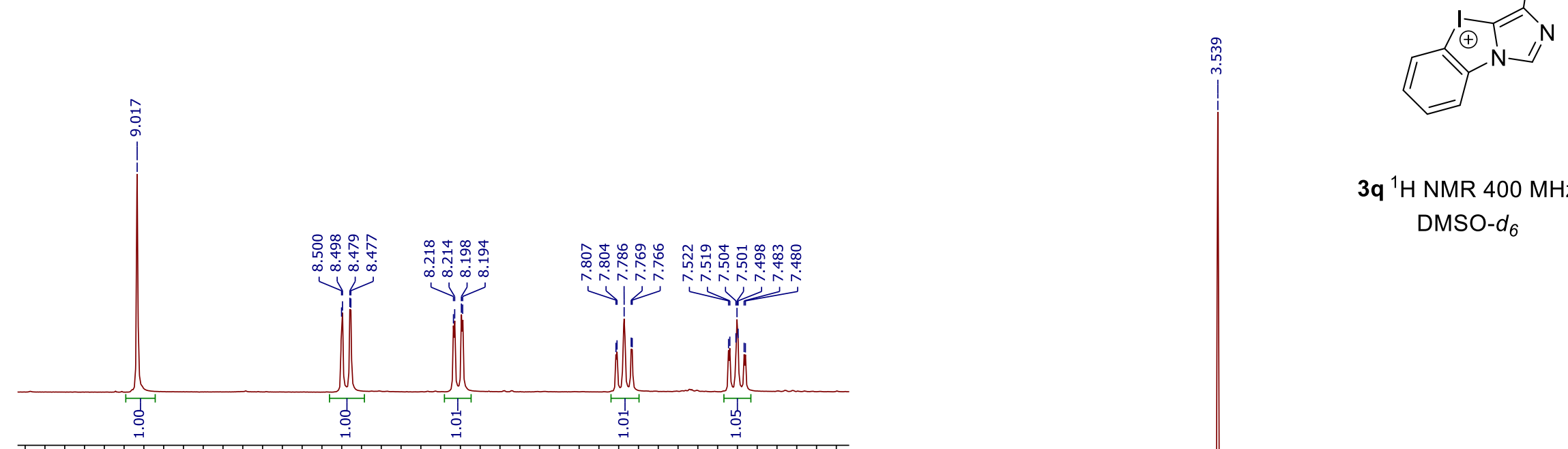

$\begin{array}{llllllllllllllllllllllllllllllll}9.3 & 9.2 & 9.1 & 9.0 & 8.9 & 8.8 & 8.7 & 8.6 & 8.5 & 8.4 & 8.3 & 8.2 & 8.1 & 8.0 & 7.9 & 7.8 & 7.7 & 7.6 & 7.5 & 7.4 & 7.3\end{array}$ f1 (ppm)

$3 q^{1} \mathrm{H}$ NMR $400 \mathrm{MHz}$

DMSO- $d_{6}$

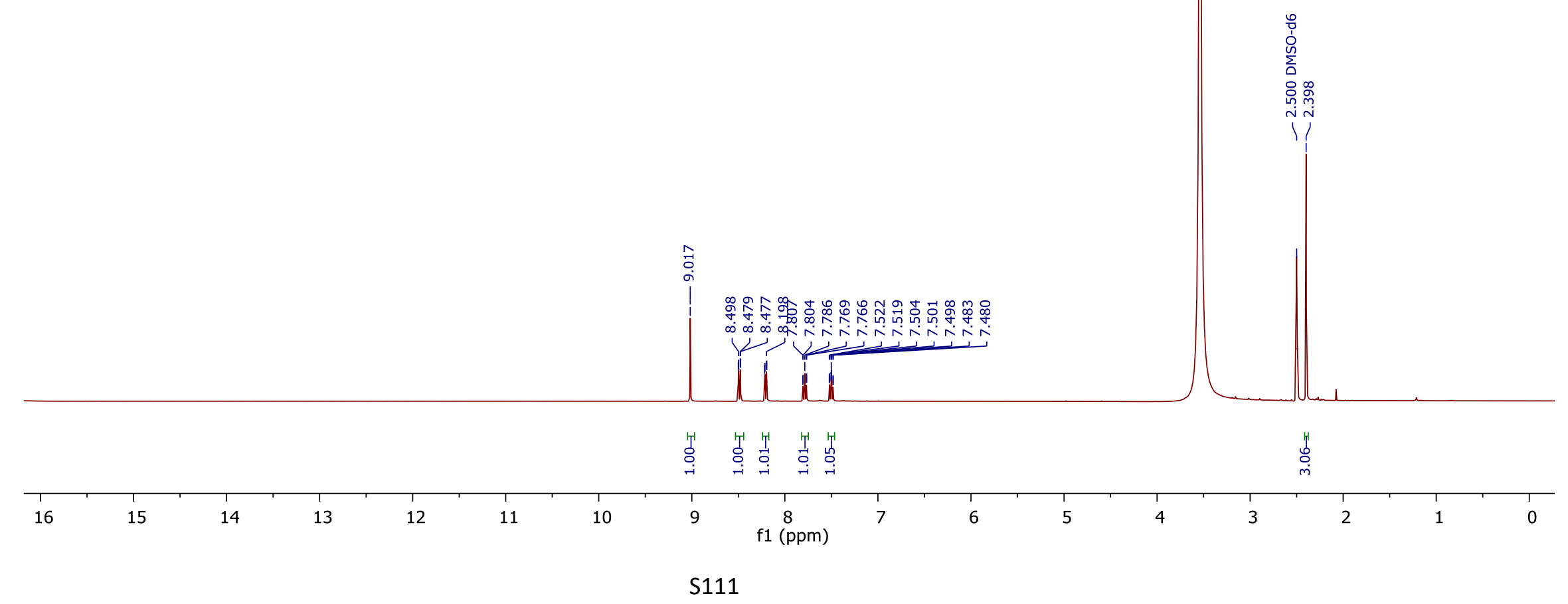




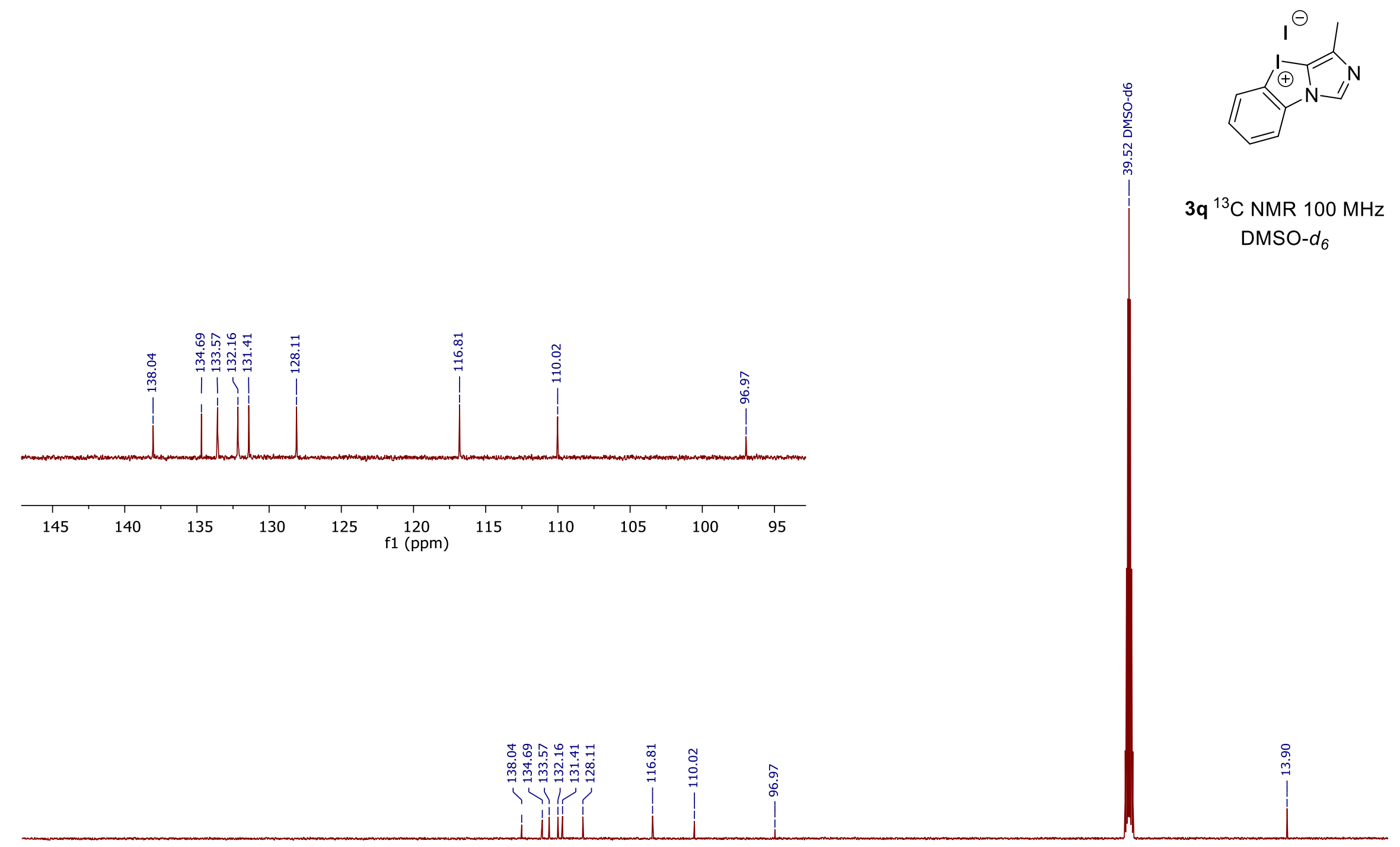

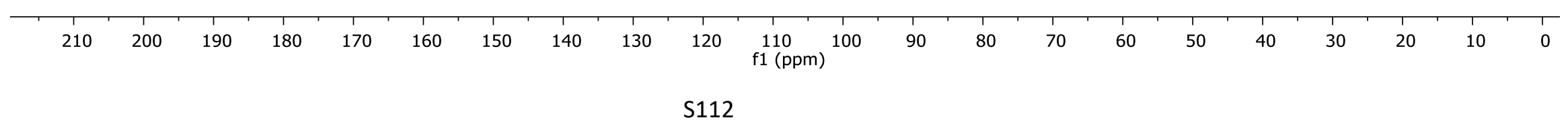




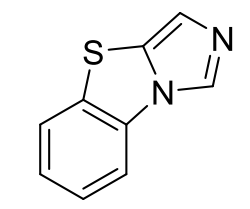

4a ${ }^{1} \mathrm{H}$ NMR $400 \mathrm{MHz}$ DMSO- $d_{6}$
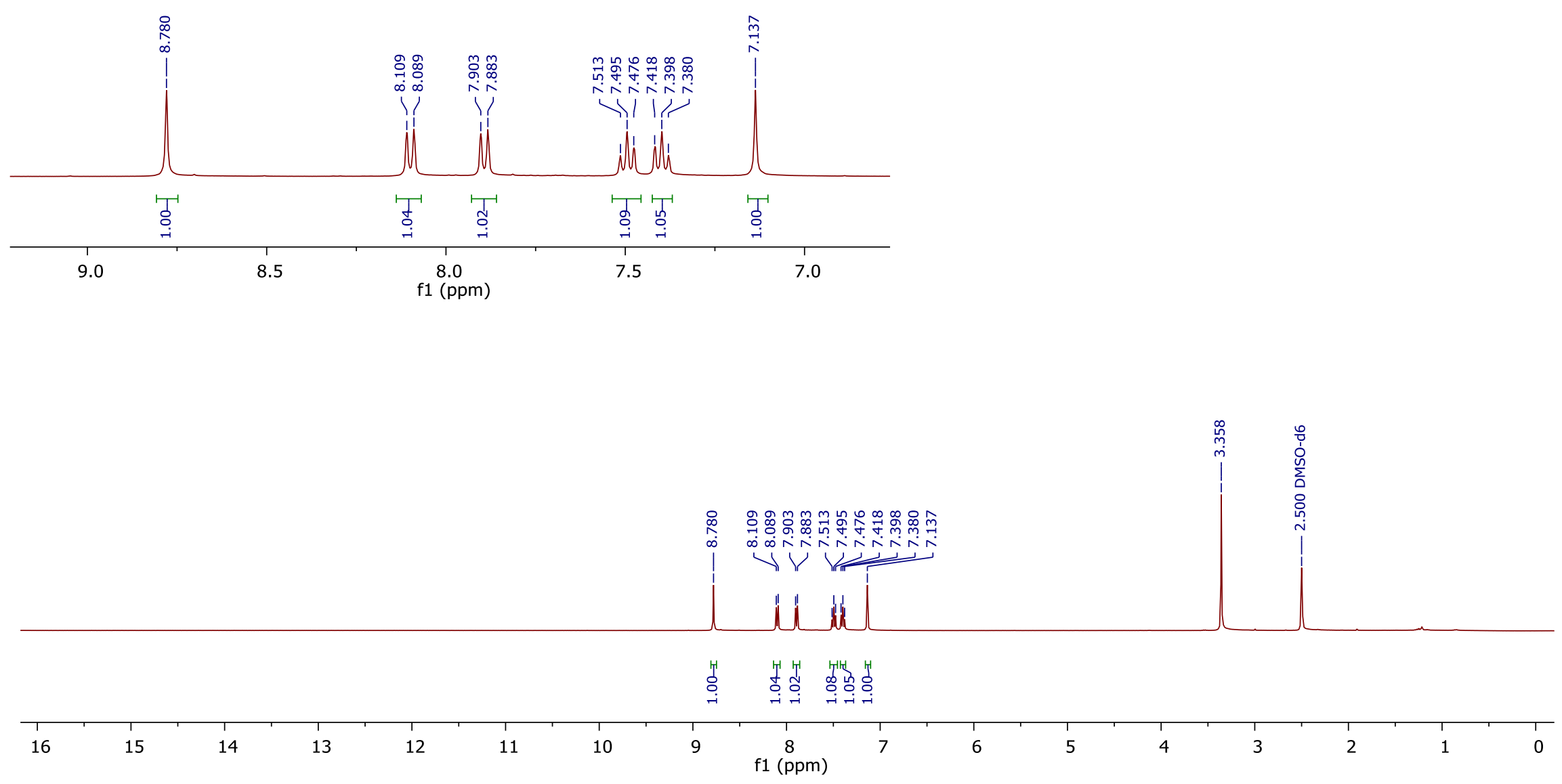


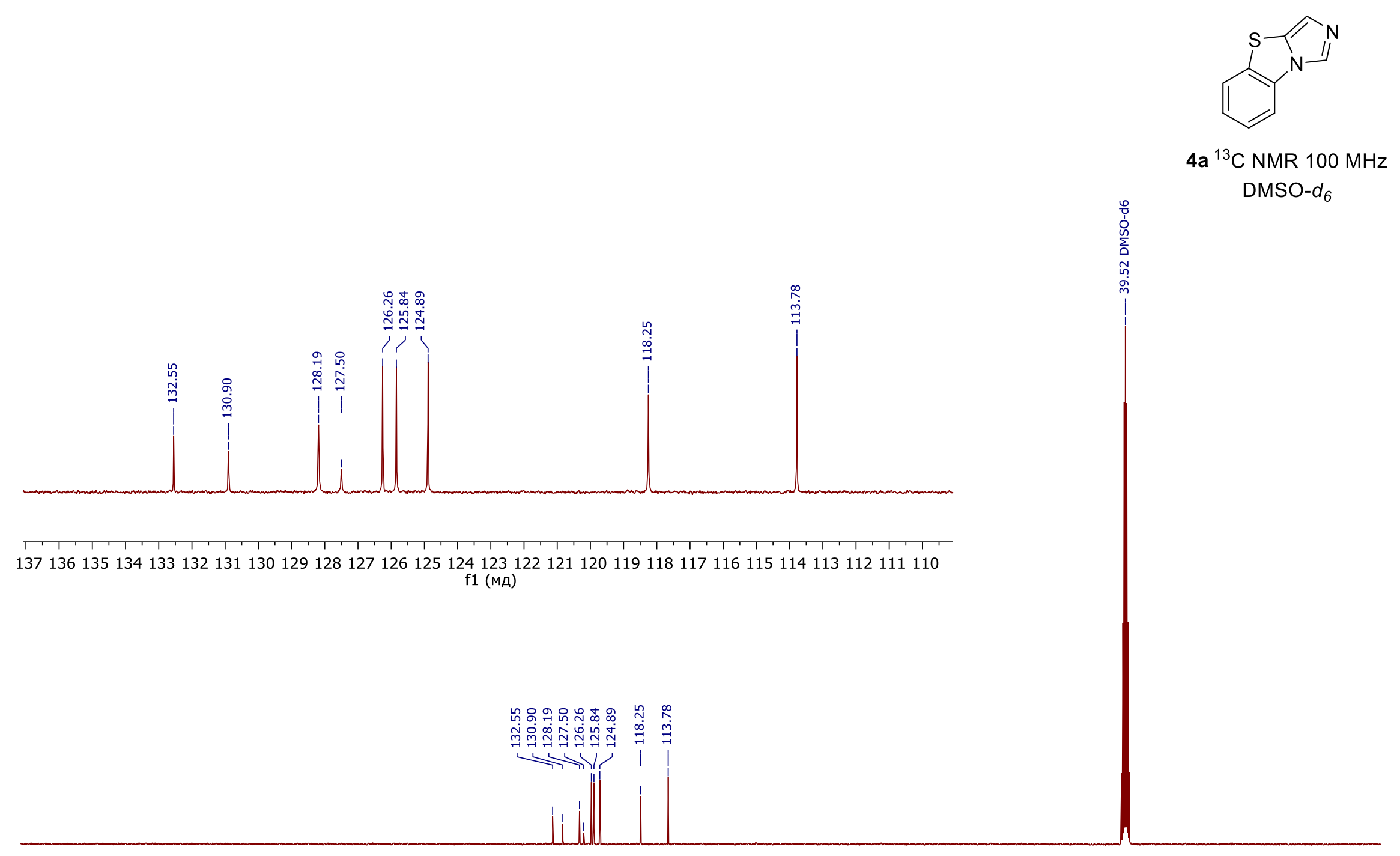

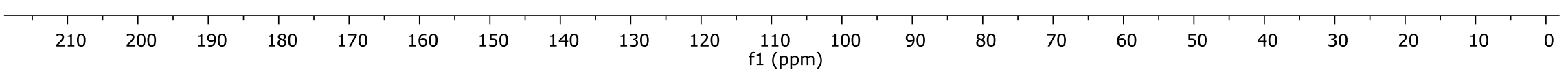




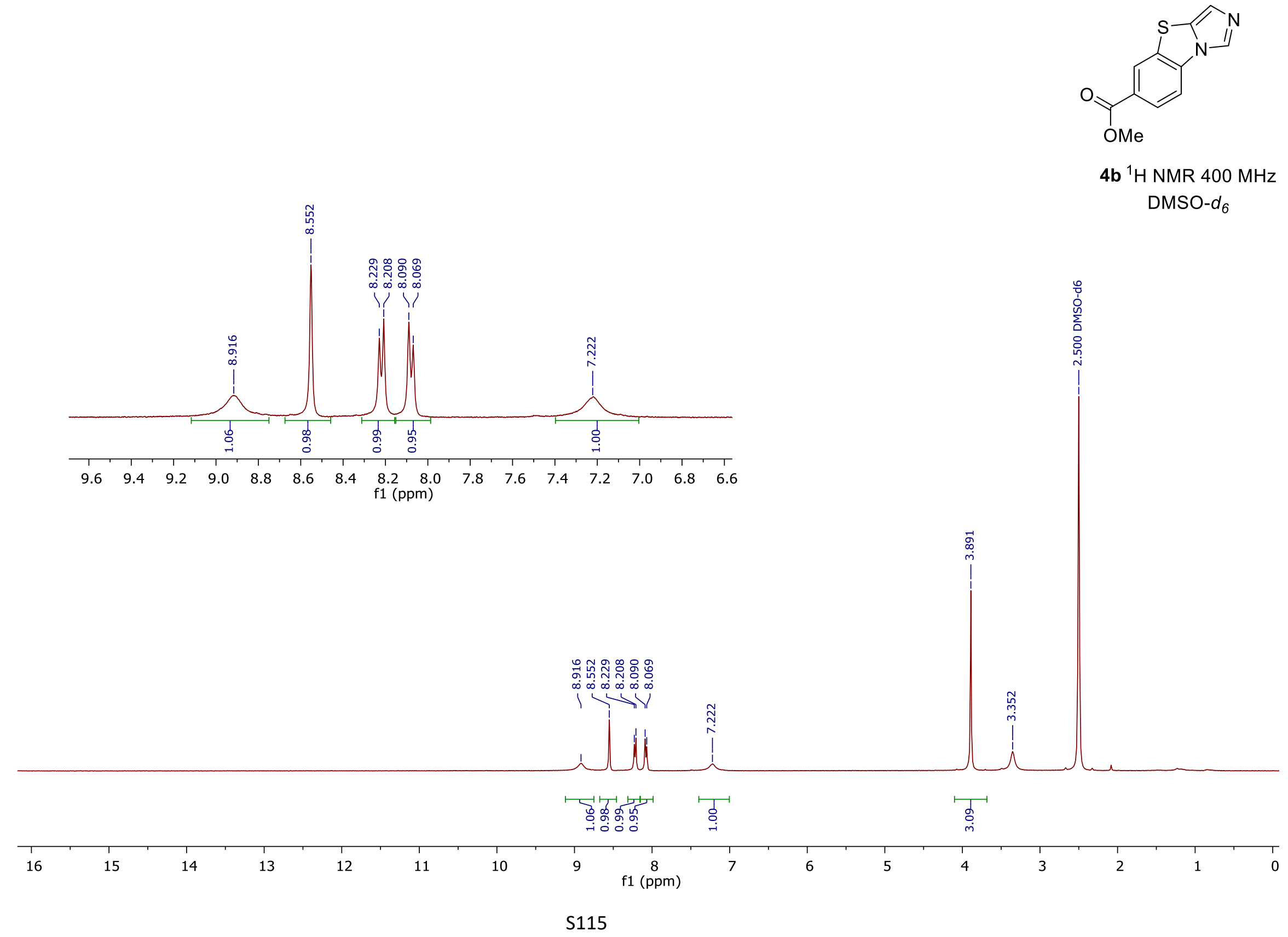



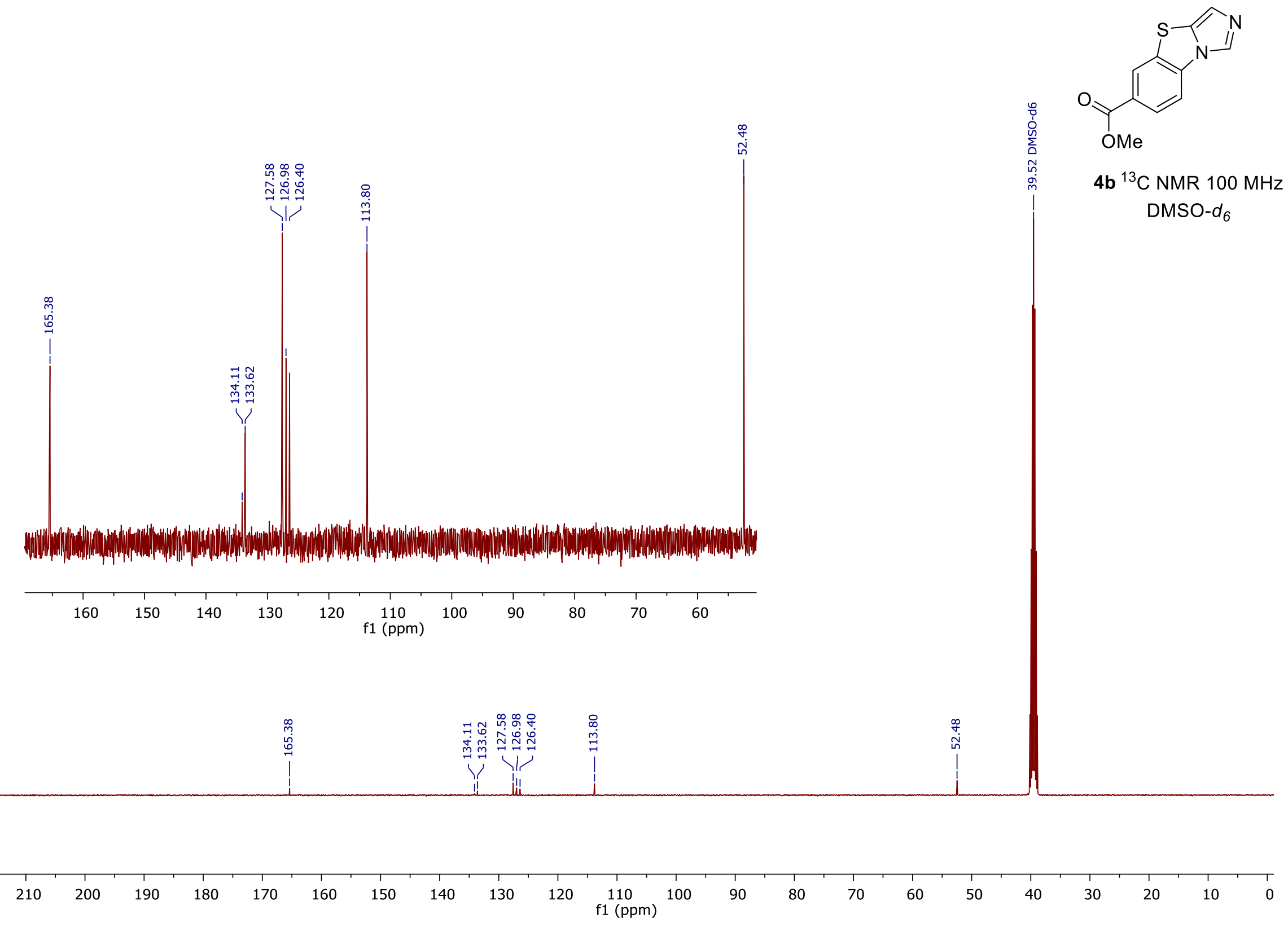


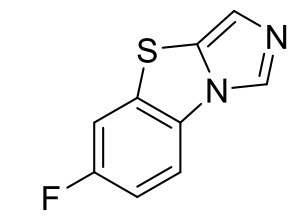

4c ${ }^{1} \mathrm{H}$ NMR $400 \mathrm{MHz}$ DMSO- $d_{6}$
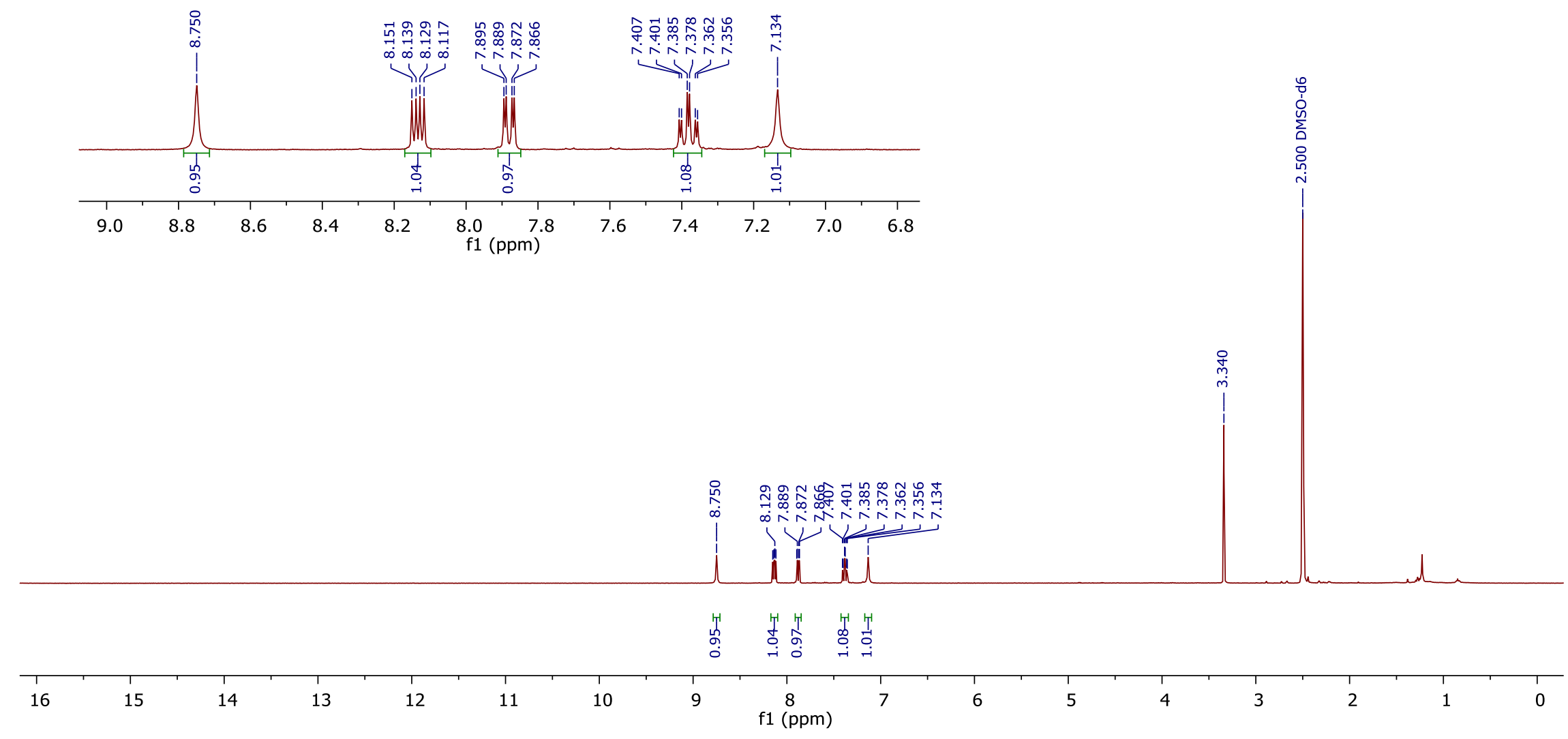

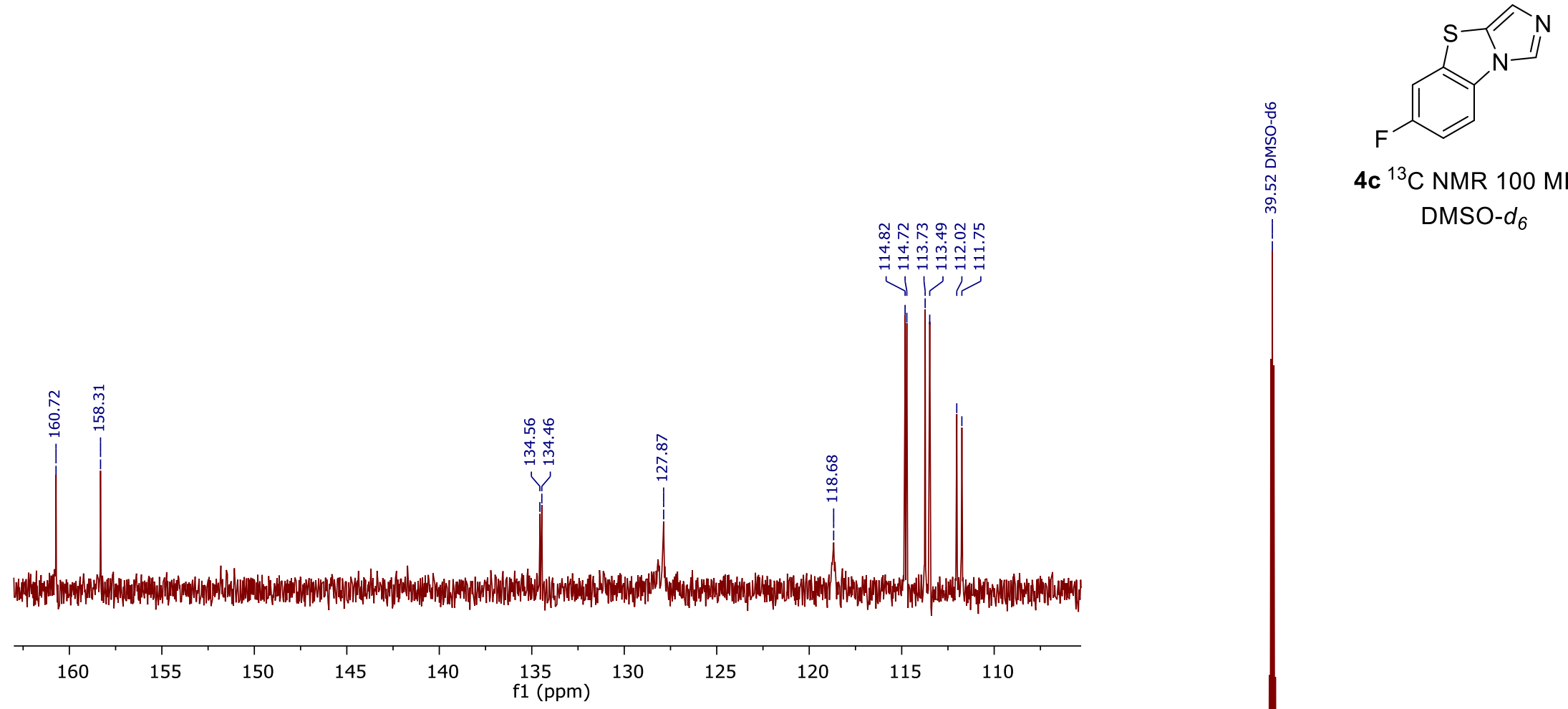

4c ${ }^{13} \mathrm{C}$ NMR $100 \mathrm{MHz}$ DMSO- $d_{6}$

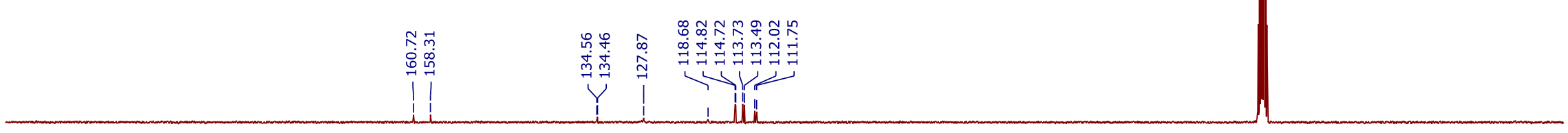

$150 \quad 140$

120

110
$\mathrm{f1}(\mathrm{ppm})$

80

$70 \quad 60$

50

40 


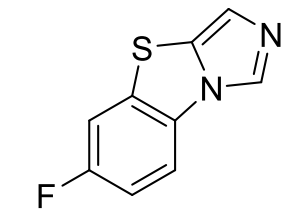

4c ${ }^{19} \mathrm{~F}$ NMR $376 \mathrm{MHz}$ DMSO-d 6

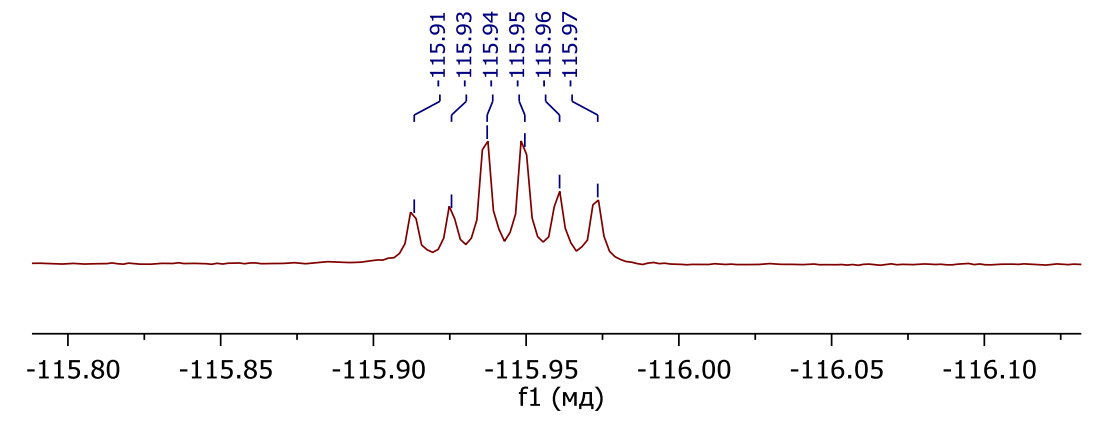

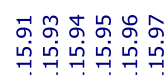

管

$\begin{array}{llllllllllllllllllllllll}10 & 0 & -10 & -20 & -30 & -40 & -50 & -60 & -70 & -80 & -90 & -100 & -110 & -120 & -130 & -140 & -150 & -160 & -170 & -180 & -190 & -200 & -210\end{array}$




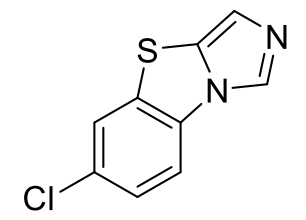

4d ${ }^{1} \mathrm{H}$ NMR $400 \mathrm{MHz}$

MeOD- $d_{4}$

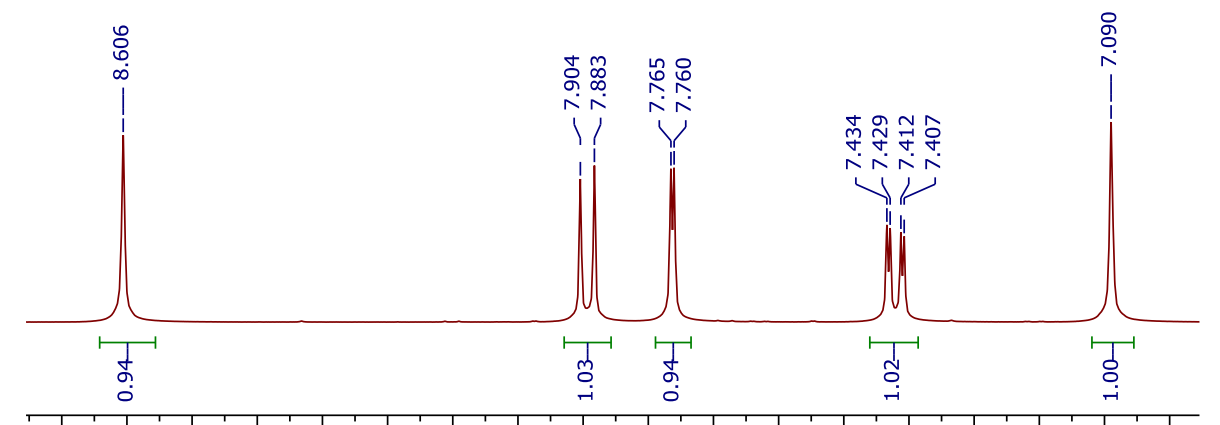

$\begin{array}{llllllllllllllllllll}8.7 & 8.6 & 8.5 & 8.4 & 8.3 & 8.2 & 8.1 & 8.0 & \begin{array}{c}7.9 \\ \text { f1 (Mम) }\end{array} & 7.7 & 7.6 & 7.5 & 7.4 & 7.3 & 7.2 & 7.1 & 7.0\end{array}$

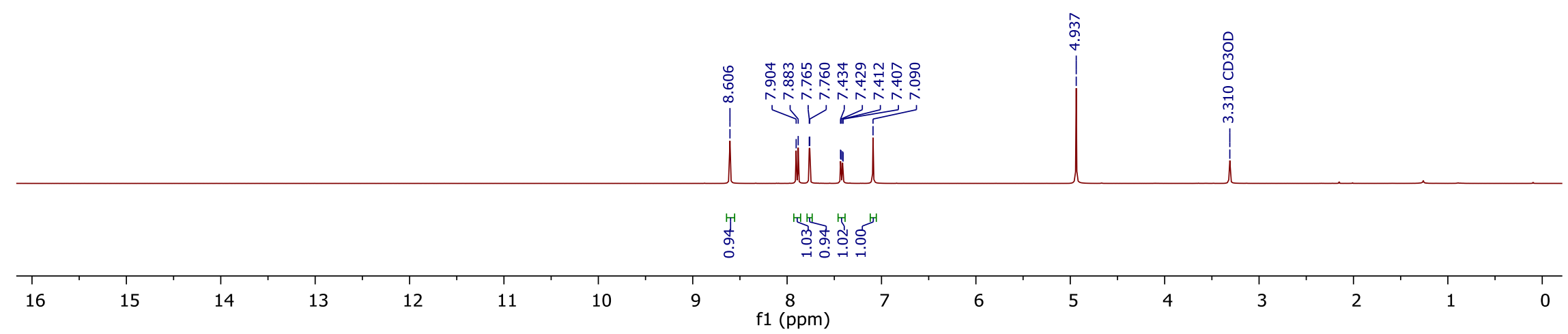



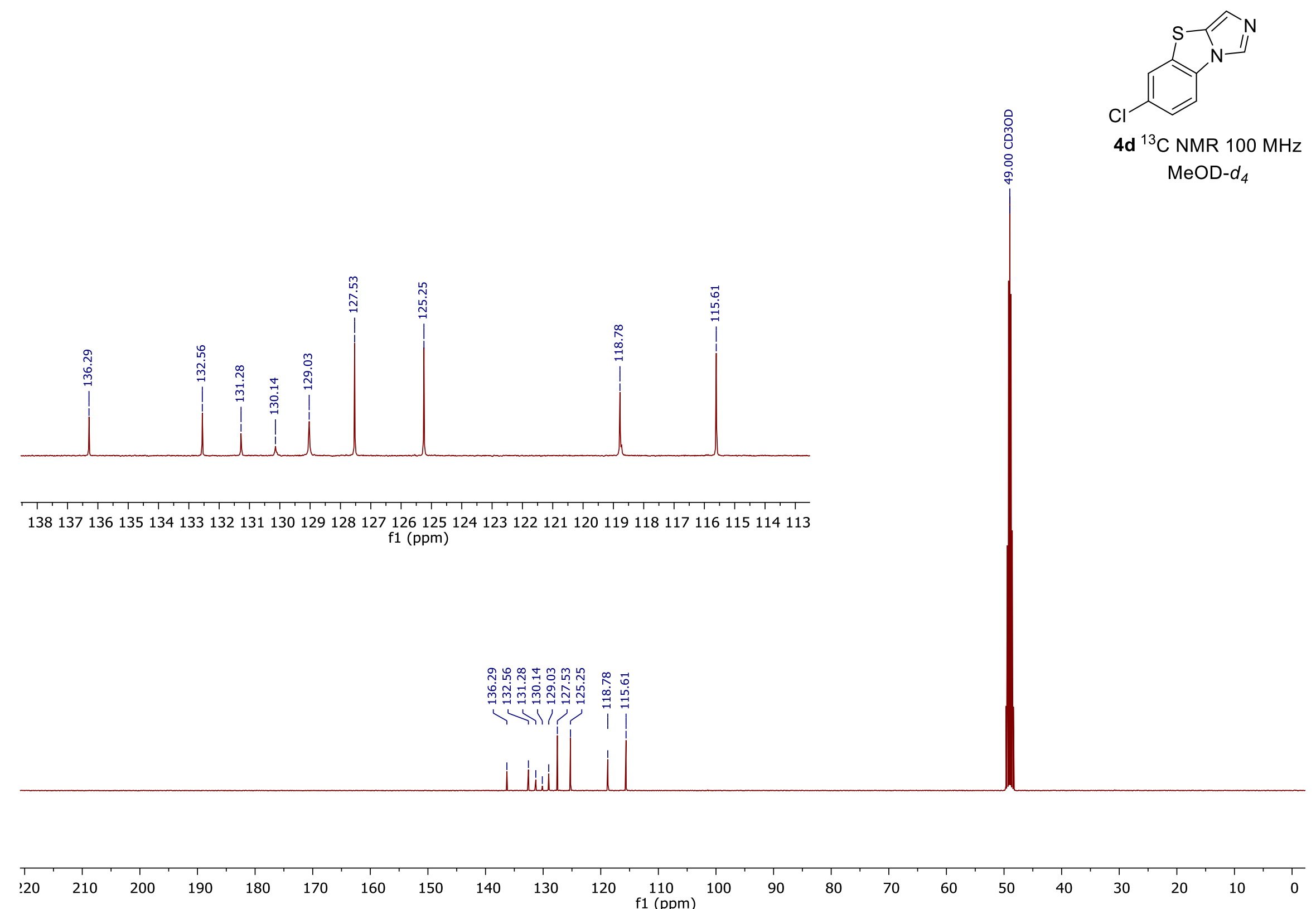


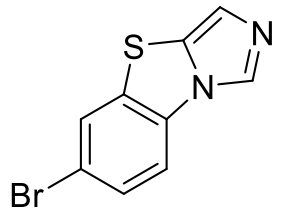

4e ${ }^{1} \mathrm{H}$ NMR $400 \mathrm{MHz}$ $\mathrm{DMSO}^{-d_{6}}$

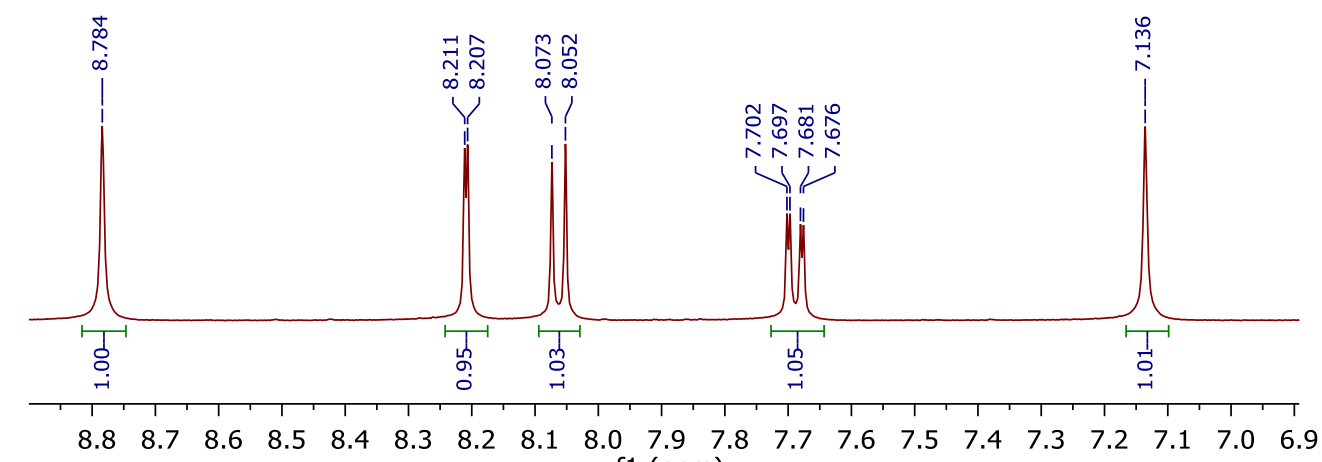
f1 (ppm)

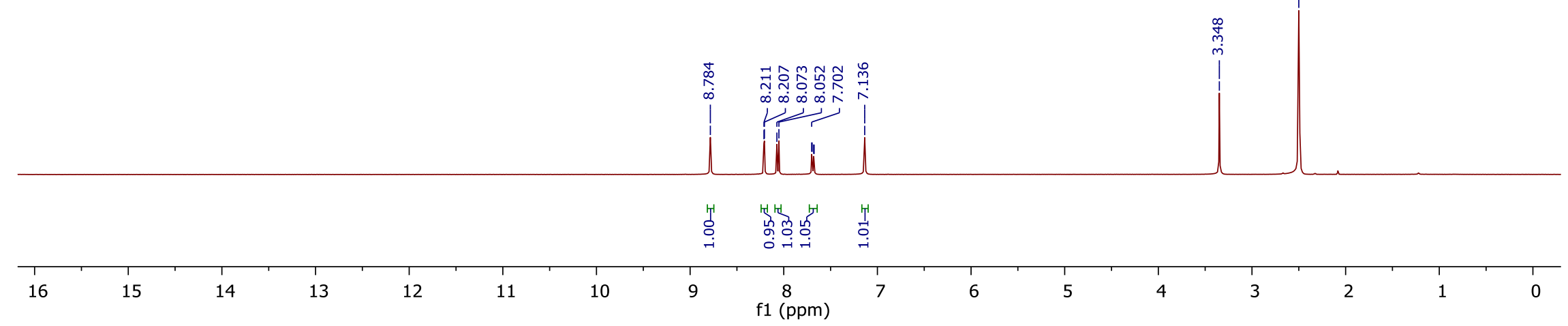




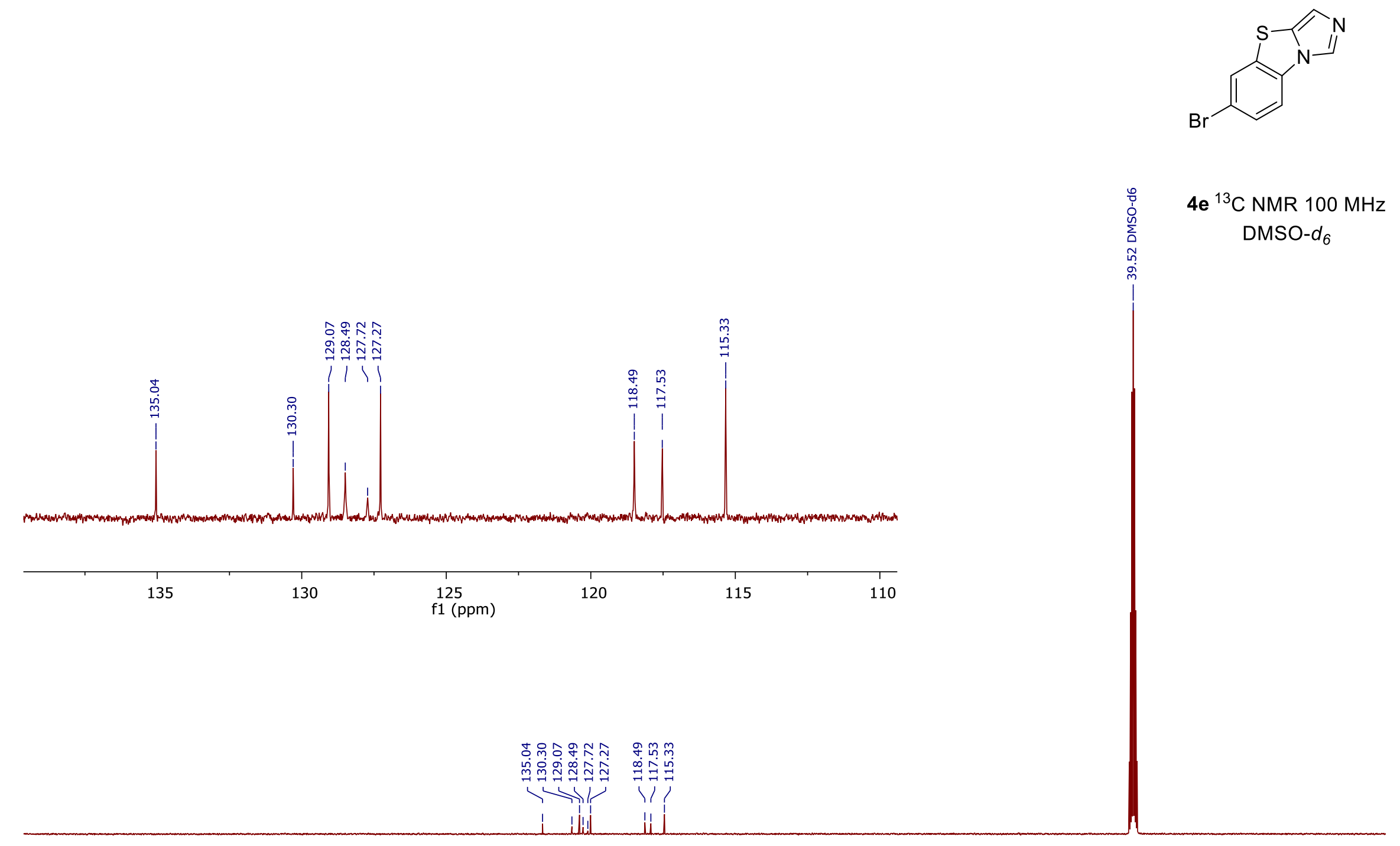

\begin{tabular}{|c|c|c|c|c|c|c|c|c|c|c|c|c|c|c|c|c|c|c|c|c|c|}
\hline 210 & 200 & $\begin{array}{c}1 \\
190\end{array}$ & 180 & 170 & 160 & 150 & 140 & 130 & 120 & $\begin{array}{c}110 \\
f 1(p p m)\end{array}$ & 100 & 90 & 80 & 70 & 60 & 50 & 40 & 30 & 20 & 10 & $\begin{array}{l}T \\
0\end{array}$ \\
\hline
\end{tabular}




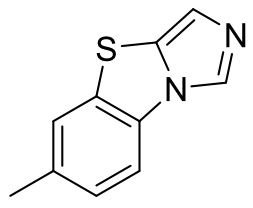

4f ${ }^{1} \mathrm{H}$ NMR $400 \mathrm{MHz}$ $\mathrm{MeOD}-d_{4}$

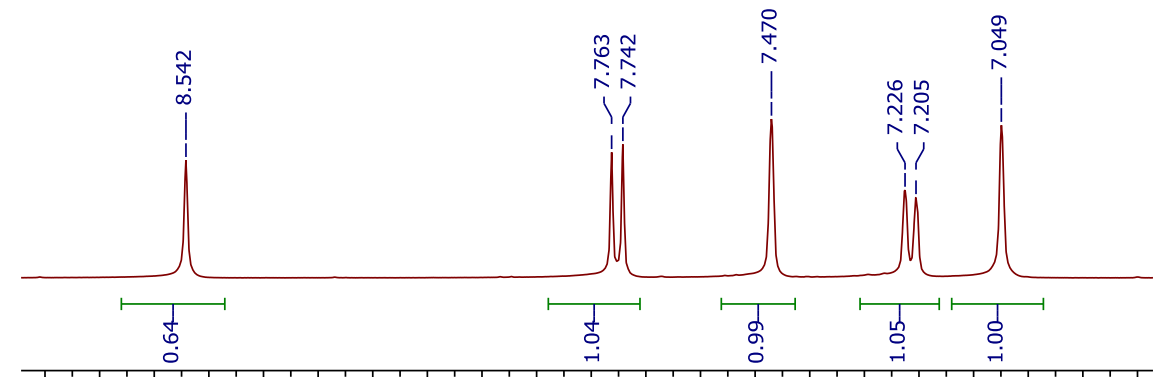

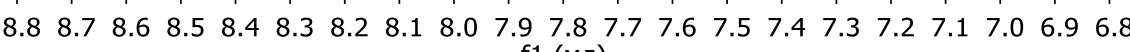
f1 (MA)

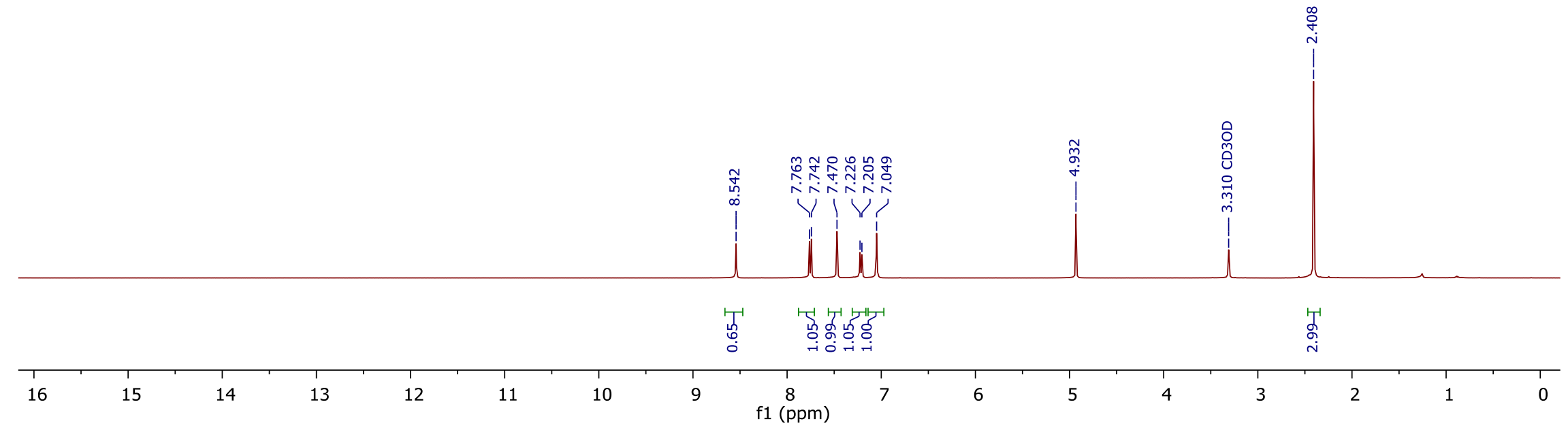



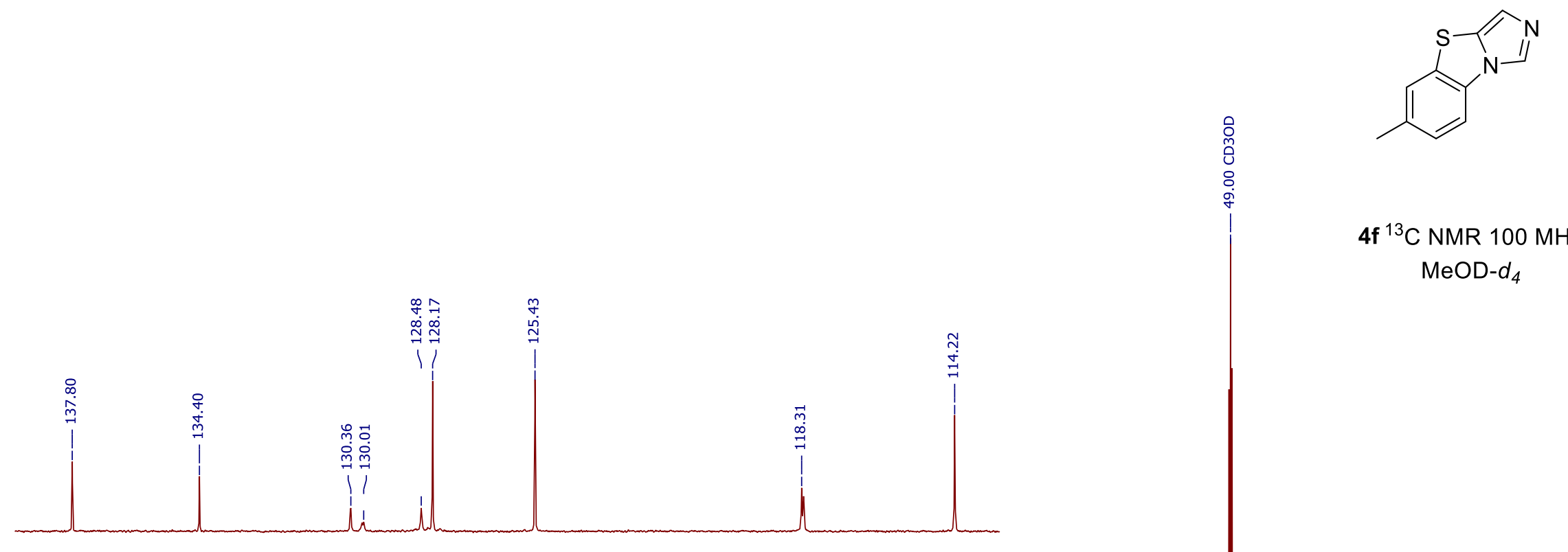

139138137136135134133132131130129128127126125124123122121120119118117116115114 f1 (ppm)

4f ${ }^{13} \mathrm{C}$ NMR $100 \mathrm{MHz}$ $\mathrm{MeOD}-d_{4}$
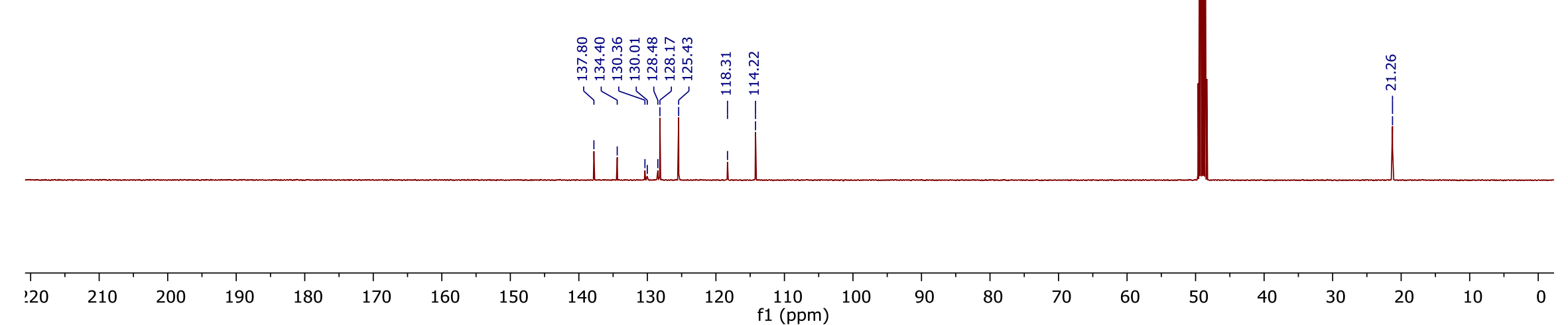


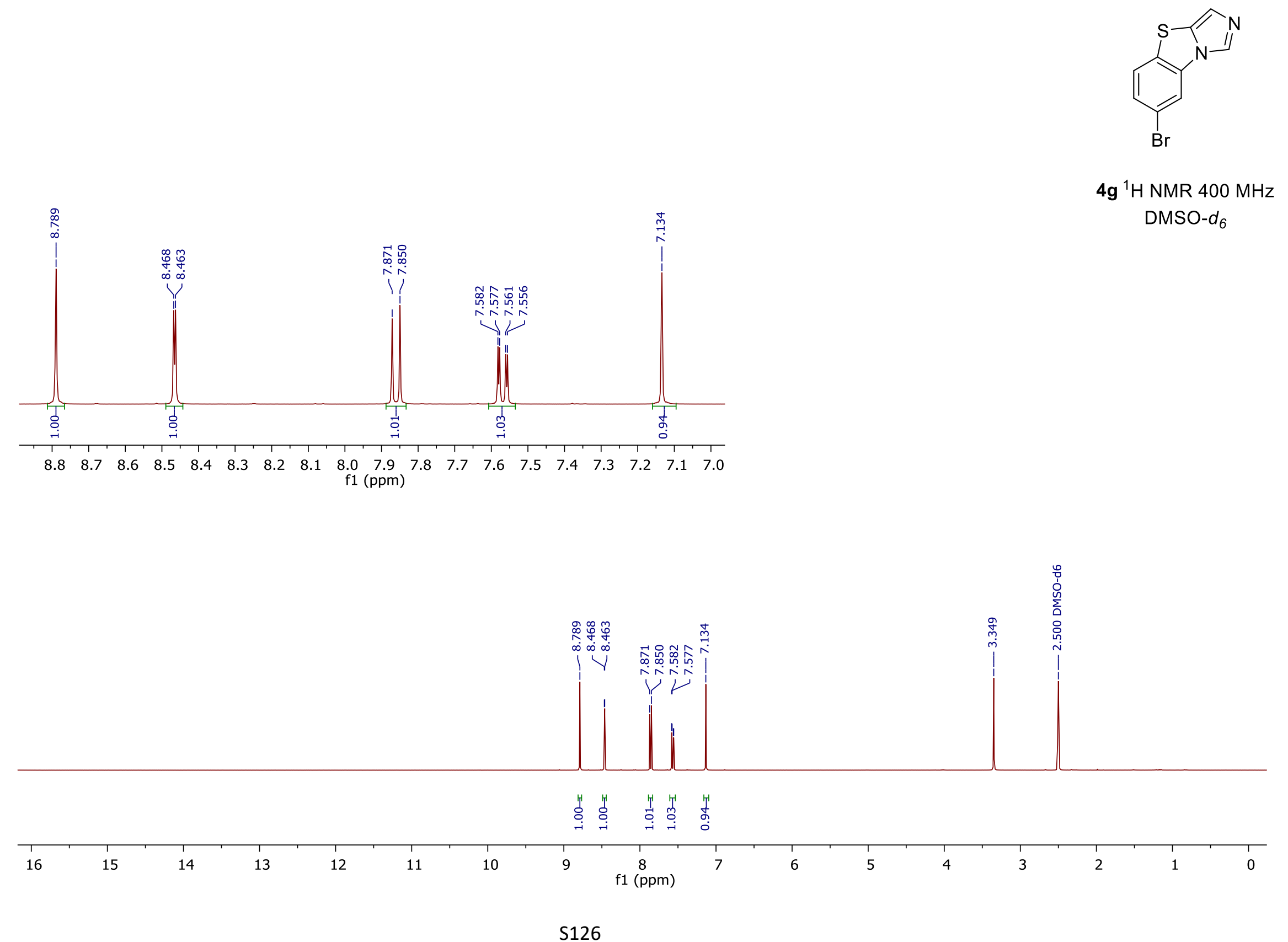




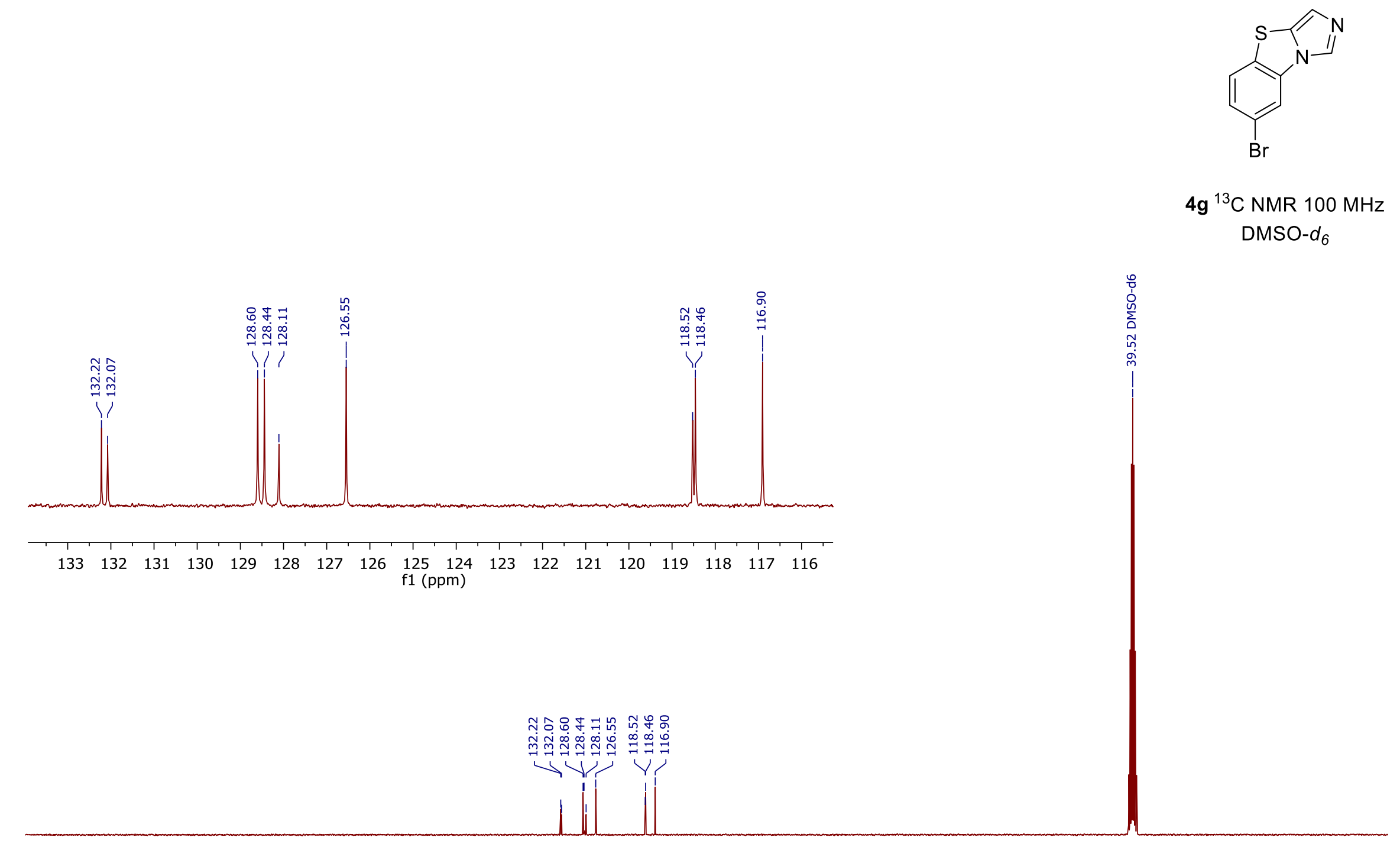

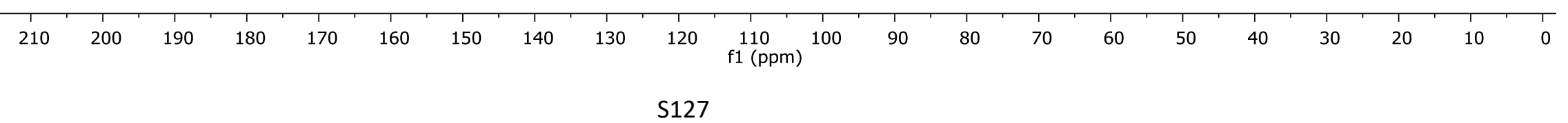




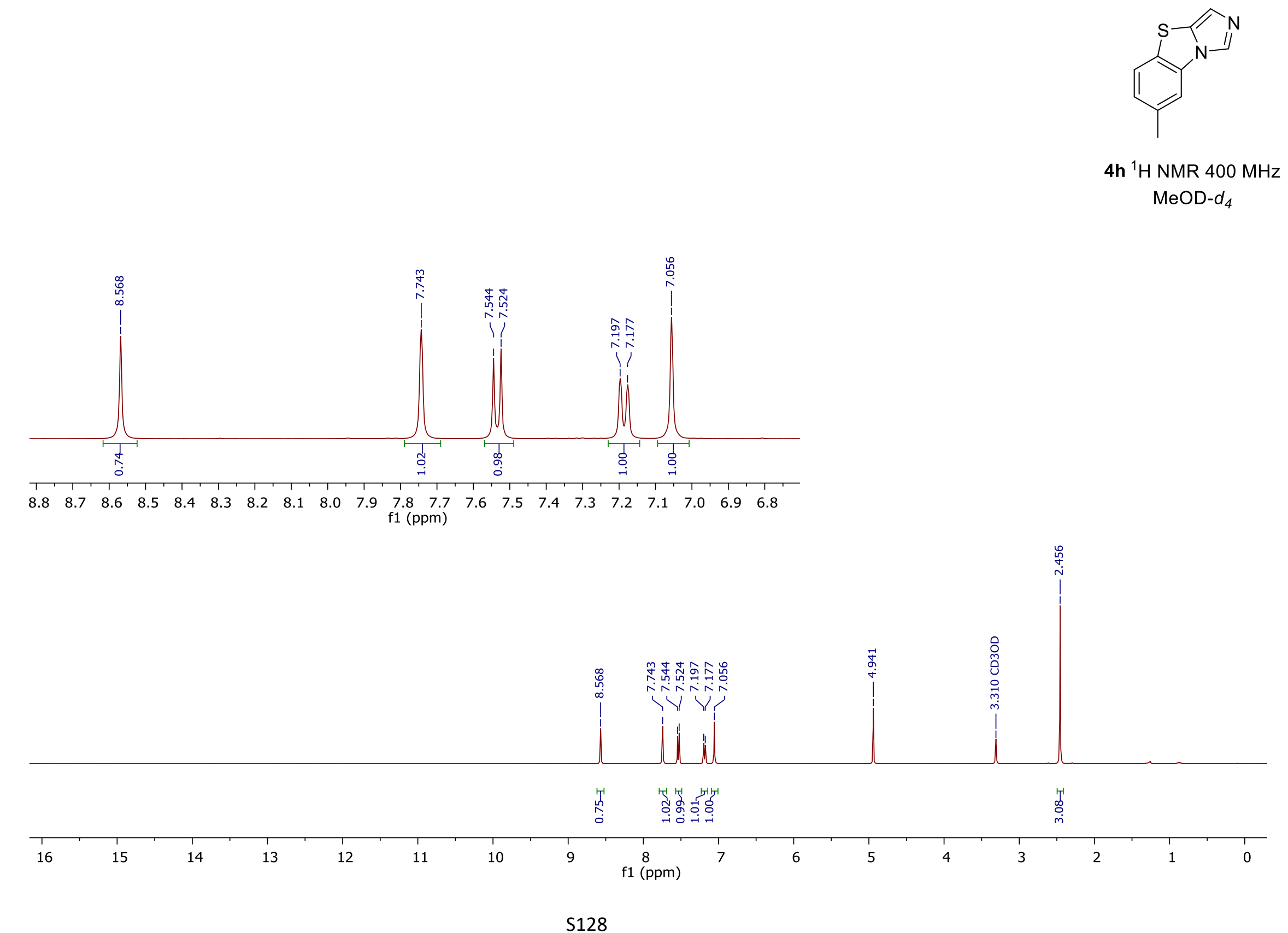



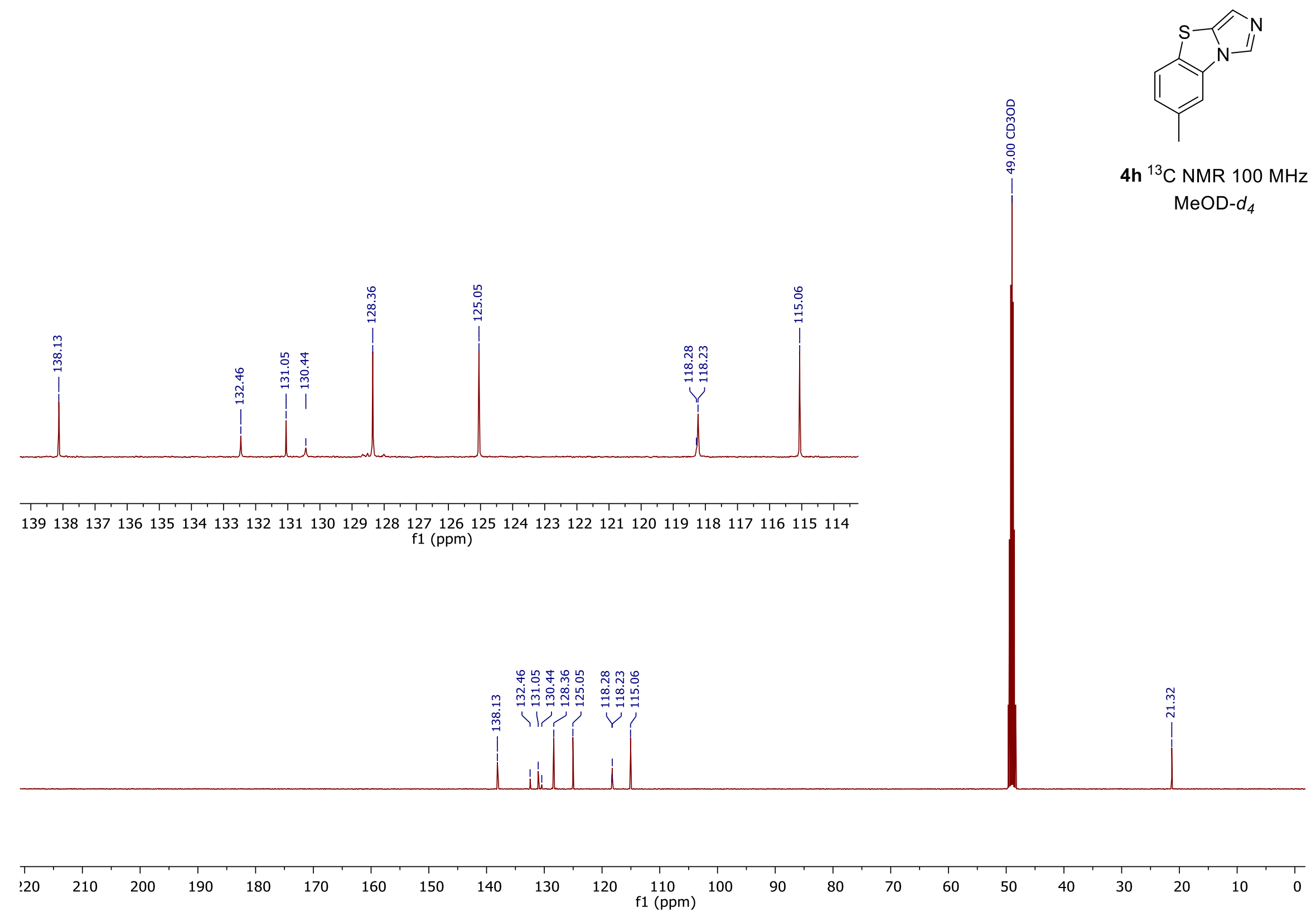


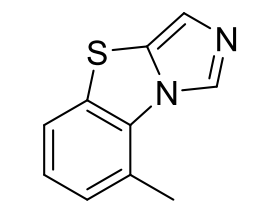

$4 i^{1} \mathrm{H}$ NMR $400 \mathrm{MHz}$ DMSO- $d_{6}$
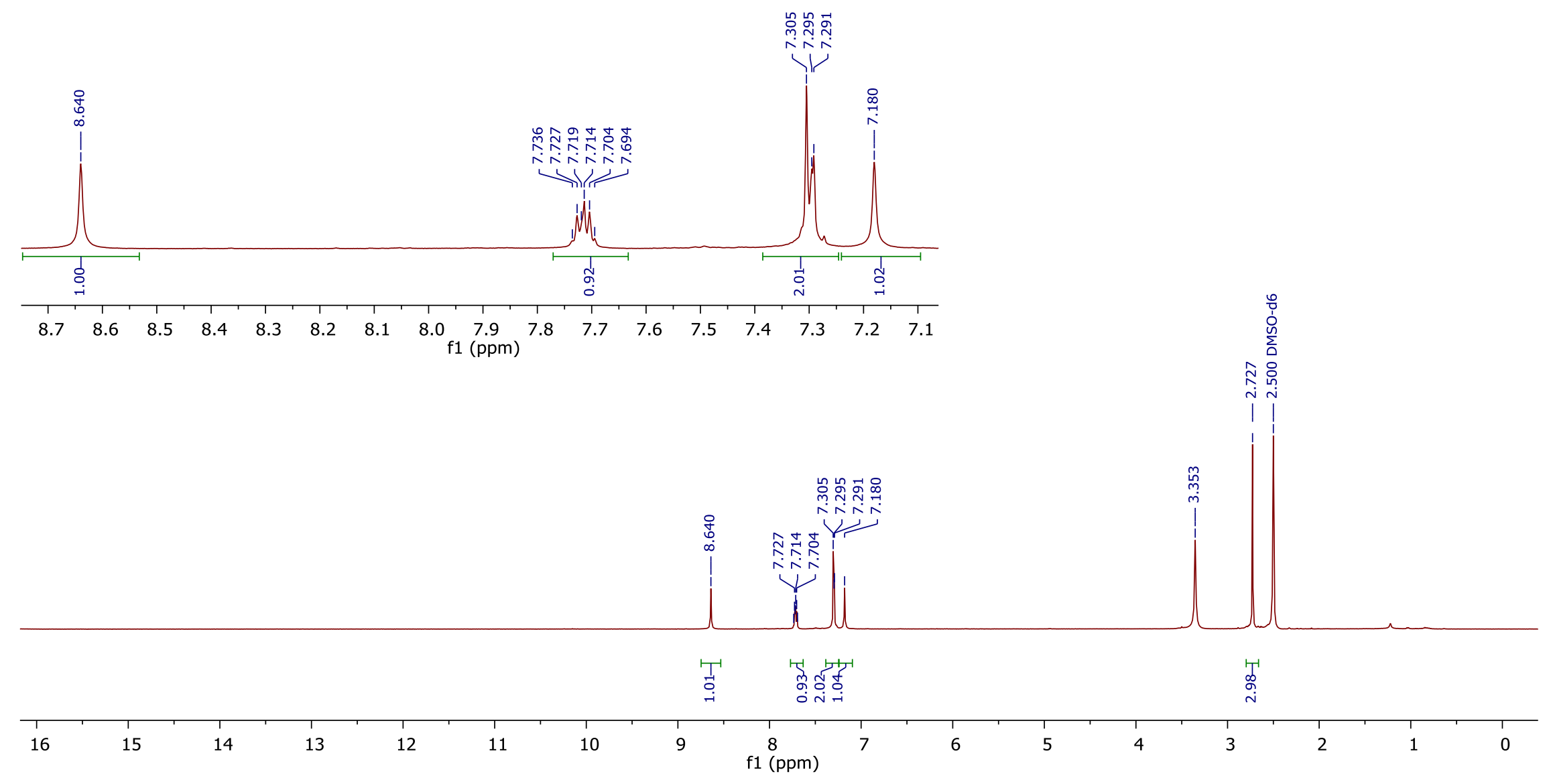


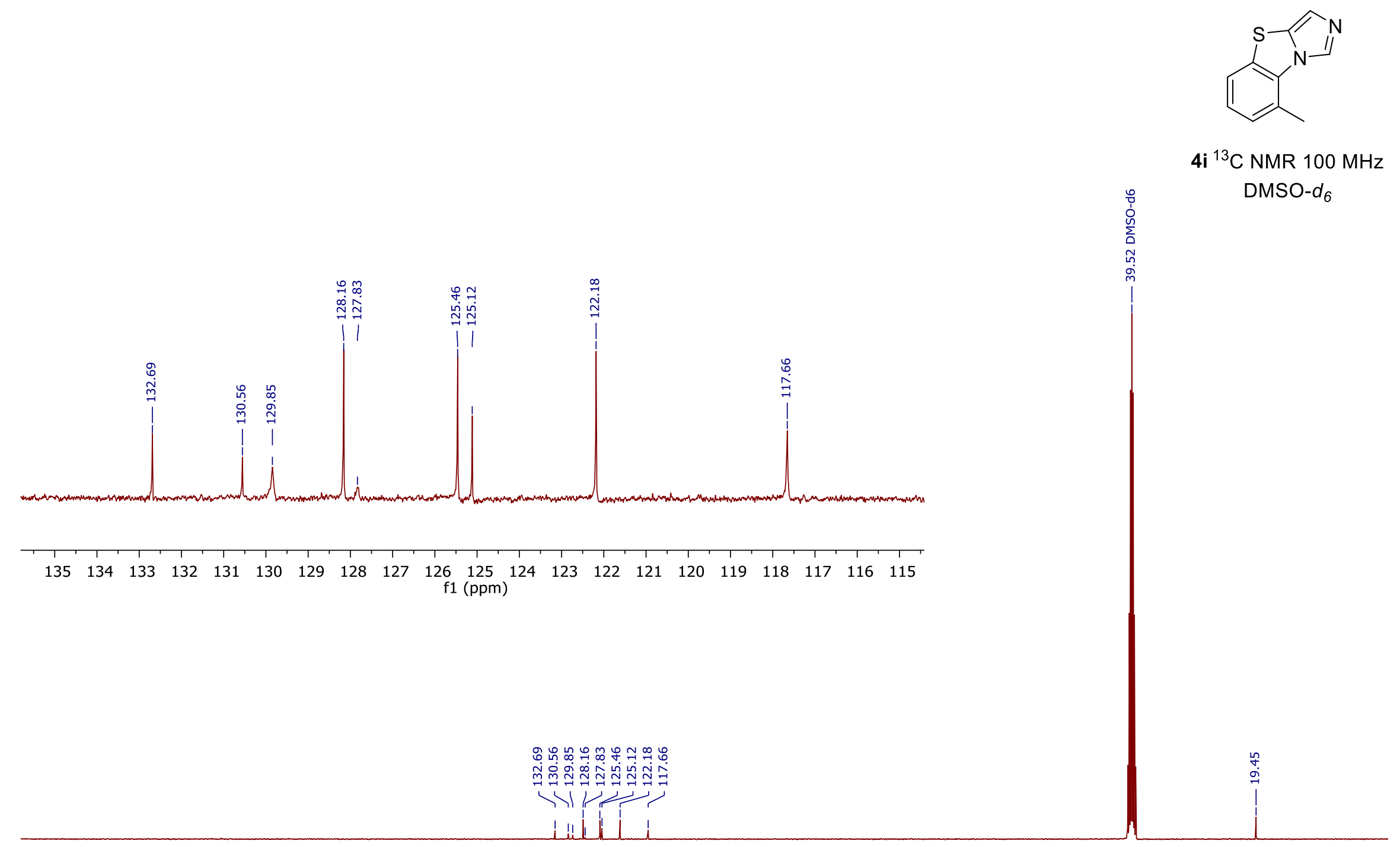

\begin{tabular}{|c|c|c|c|c|c|c|c|c|c|c|c|c|c|c|c|c|c|c|c|c|c|}
\hline 210 & 200 & 190 & 180 & 170 & 160 & 150 & 140 & 130 & 120 & $\begin{array}{c}110 \\
\text { f1 (ppm) }\end{array}$ & 100 & 90 & 80 & 70 & 60 & 50 & 40 & 30 & 20 & 10 & 0 \\
\hline
\end{tabular}




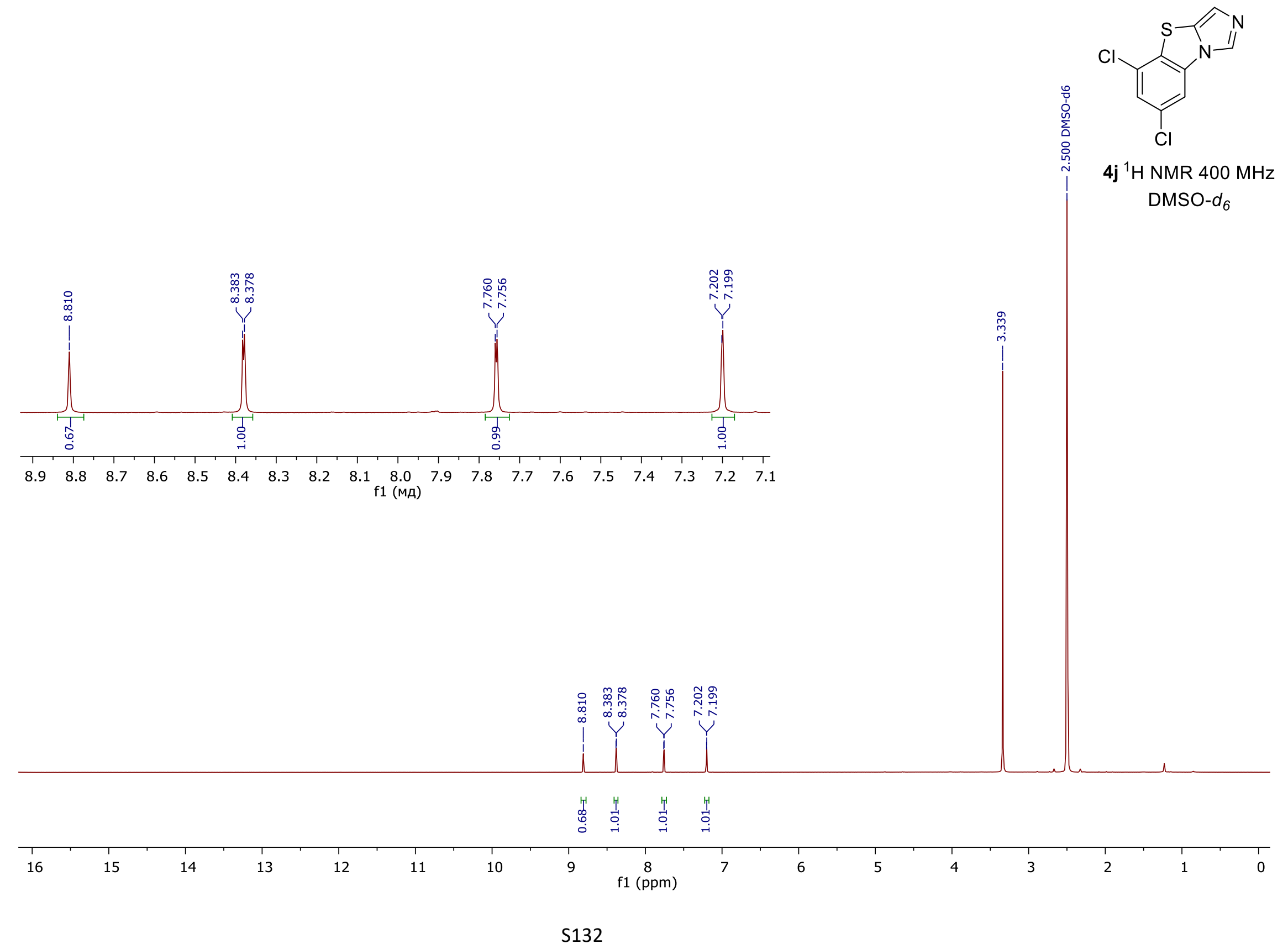



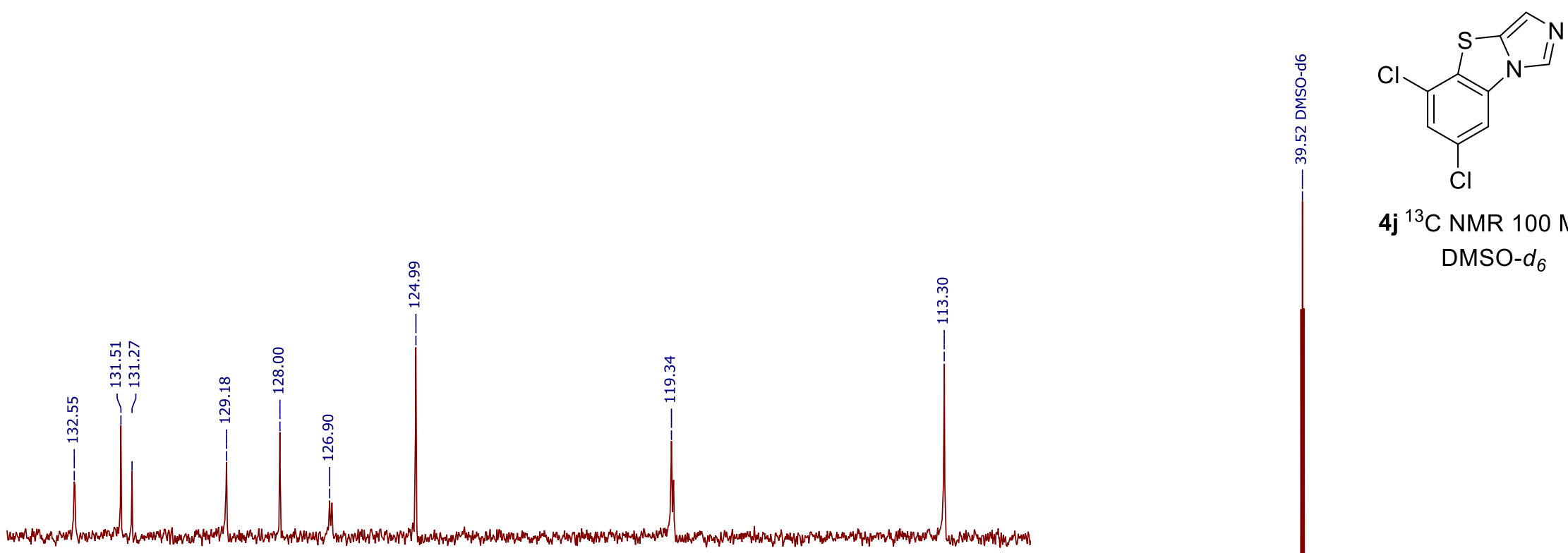

4j ${ }^{13} \mathrm{C}$ NMR $100 \mathrm{MHz}$ DMSO- $d_{6}$ f1 (MA)

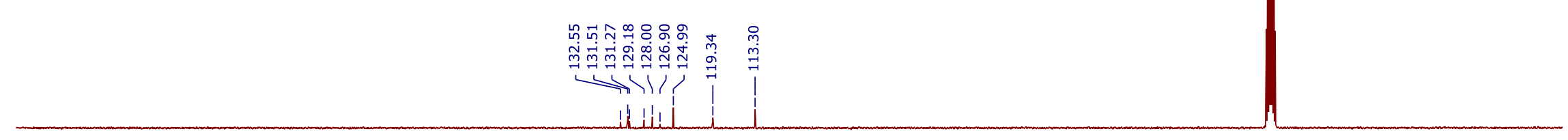

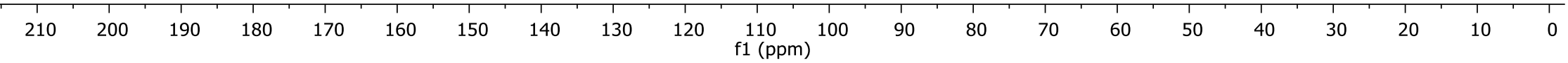



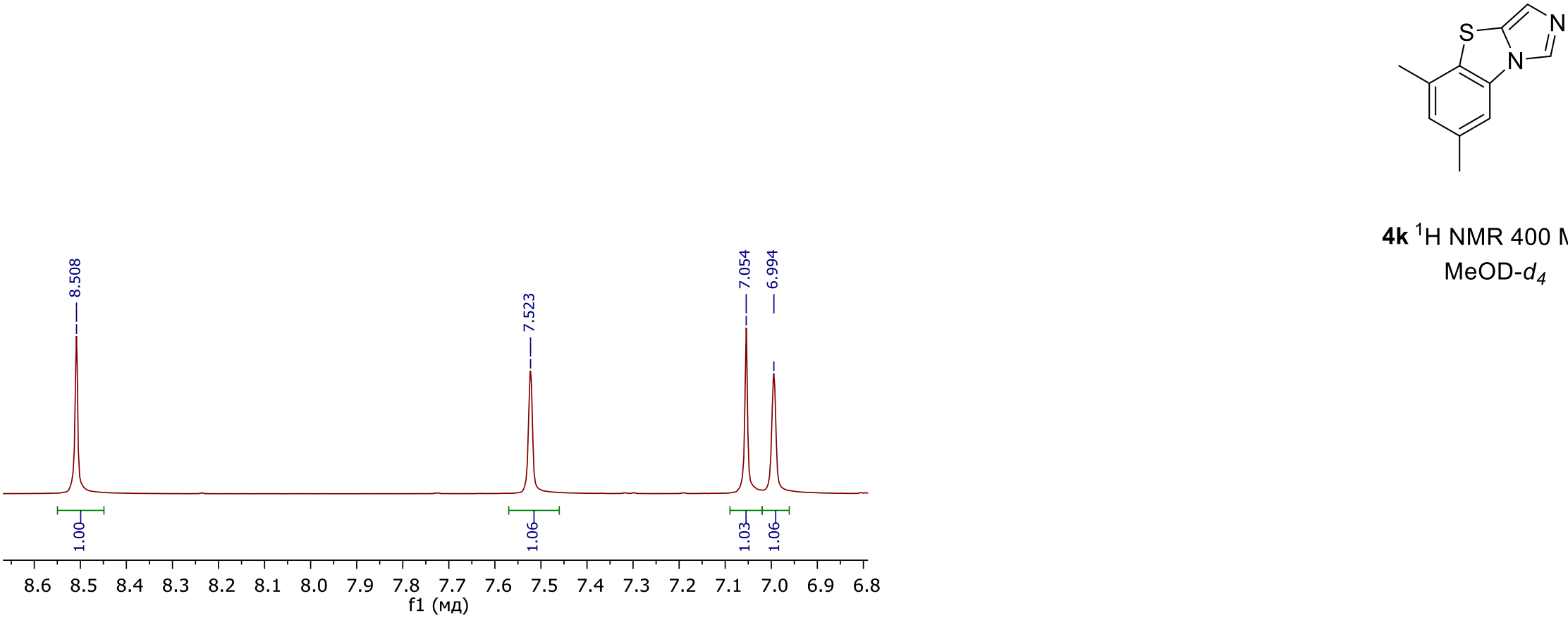

4k ${ }^{1} \mathrm{H}$ NMR $400 \mathrm{MHz}$

MeOD- $d_{4}$

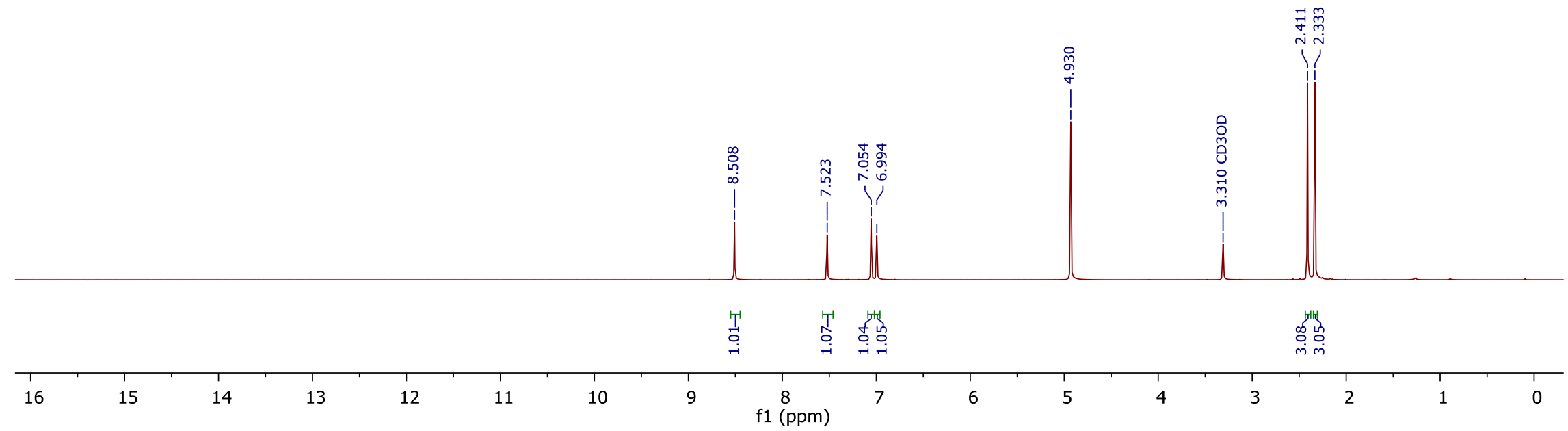



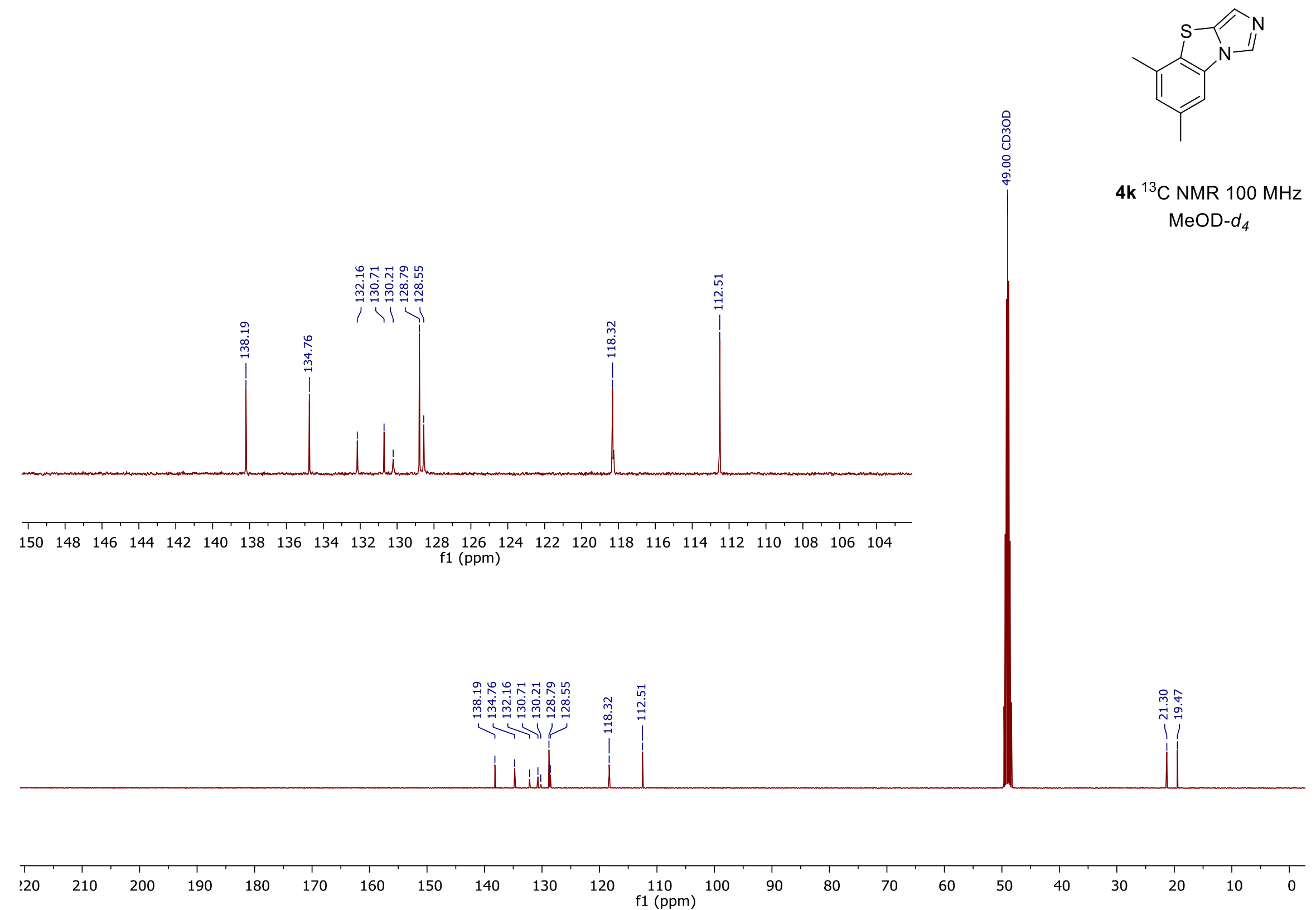